Environmental Molecular

Sciences Laboratory

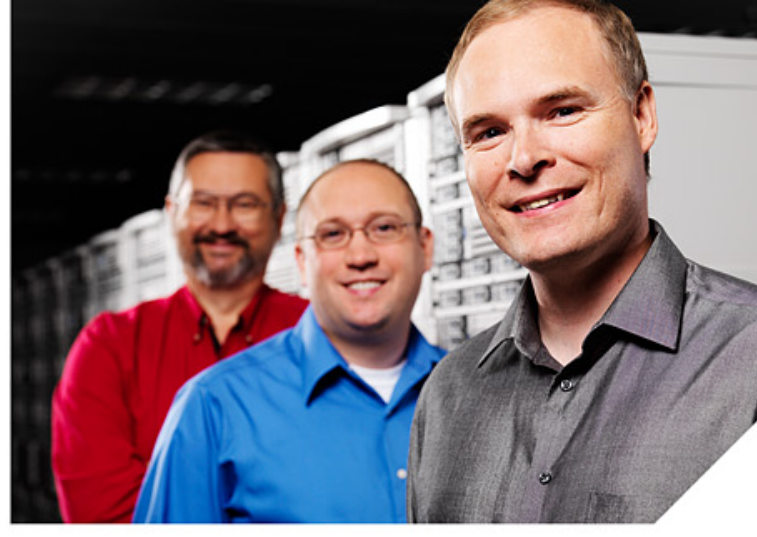

EMSL Fiscal Year

2009 Annual Report
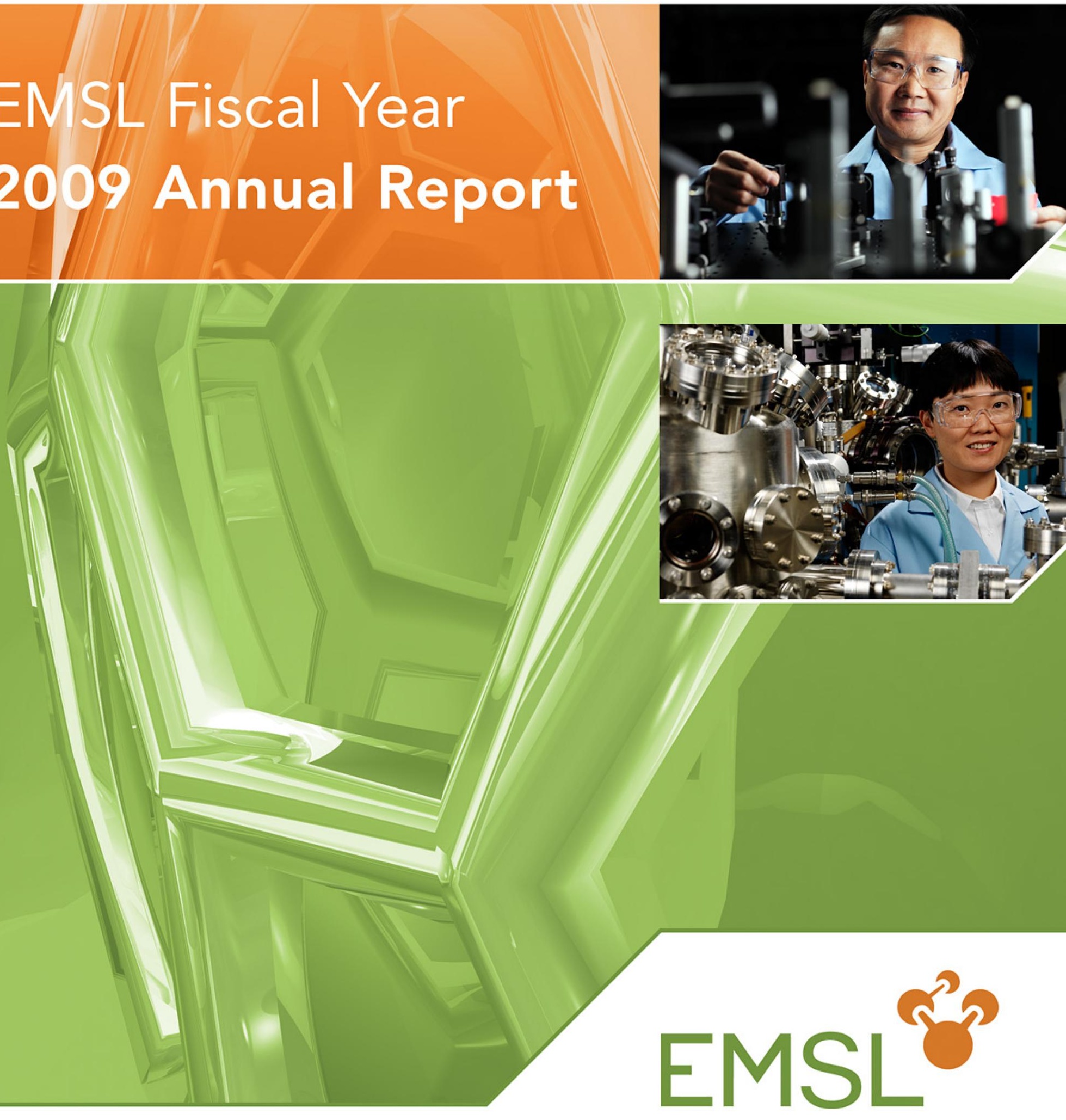


\title{
DISCLAIMER
}

This report was prepared as an account of work sponsored by an agency of the United States Government. Neither the United States Government nor any agency thereof, nor Battelle Memorial Institute, nor any of their employees, makes any warranty, express or implied, or assumes any legal liability or responsibility for the accuracy, completeness, or usefulness of any information, apparatus, product, or process disclosed, or represents that its use would not infringe privately owned rights. Reference herein to any specific commercial product, process, or service by trade name, trademark, manufacturer, or otherwise does not necessarily constitute or imply its endorsement, recommendation, or favoring by the United States Government or any agency thereof, or Battelle Memorial Institute. The views and opinions of authors expressed herein do not necessarily state or reflect those of the United States Government or any agency thereof.

\author{
PACIFIC NORTHWEST NATIONAL LABORATORY \\ operated by \\ BATTELLE \\ for the \\ UNITED STATES DEPARTMENT OF ENERGY \\ under Contract DE-AC05-76RL01830
}

Printed in the United States of America
Available to DOE and DOE contractors from the Office of Scientific and Technical Information,
P.O. Box 62, Oak Ridge, TN 37831-0062;
ph: (865) 576-8401
fax: $(865) 576-5728$
email: reports@adonis.osti.gov

\begin{abstract}
Available to the public from the National Technical Information Service, U.S. Department of Commerce, 5285 Port Royal Rd., Springfield, VA 22161 ph: (800) 553-6847 fax: (703) 605-6900 email: orders@ntis.fedworld.gov

online ordering: http://www.ntis.gov/ordering.htm
\end{abstract}

This document was printed on recycled paper. 
PNNL-19196

\section{EMSL 2009 Annual Report}

MA Showalter

LE Kathmann

KL Manke

JG Wiley

JR Reed

February 2010

Prepared for the U.S. Department of Energy's Office of Biological and Environmental Research under Contract DE-AC05-76RL01830

Pacific Northwest National Laboratory

Richland, Washington 99352 


\section{Contents}

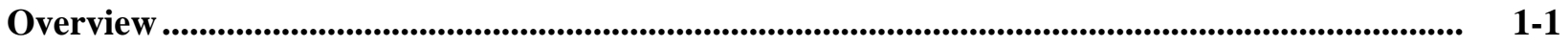

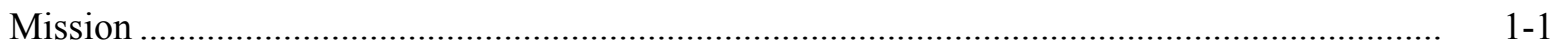

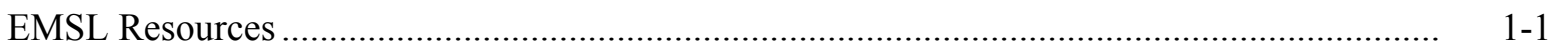

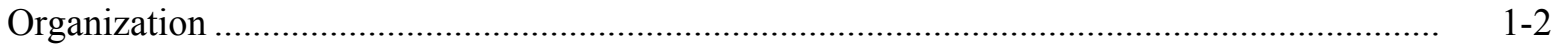

Science Advisory Committee ………………………………......................................... $1-2$

User Advisory Committee ............................................................................................

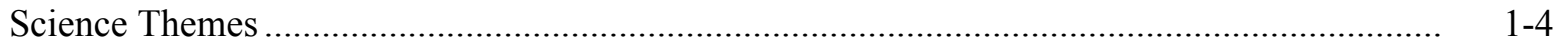

Proposals and Scientific User Access Modes ........................................................................ 1-4

Calls for Proposal Focus .................................................................................... $1-4$

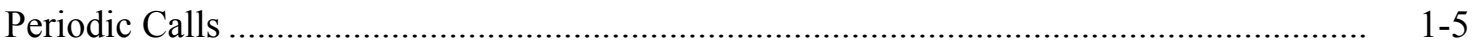

EMSL Highlights ................................................................................................

Major EMSL Activities ........................................................................................... 1-8

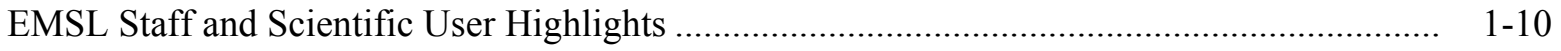

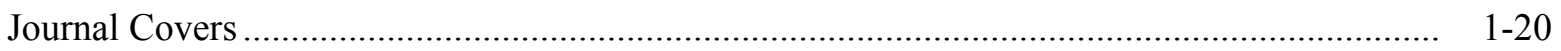

Outreach Activities .................................................................................................... 1-27

Biological Interactions and Dynamics................................................................................................... 2-1-1

De novo Sequencing of Unique Sequence Tags for Discovery of Post-Translational

Modifications of Proteins ...................................................................................... 2-1-2

The Effects of Aging on the Luminescence of P5EG-Coated Water-Soluble ZnO

Nanoparticles Solutions........................................................................................... 2-1-3

Automated Gain Control Ion Funnel Trap for Orthogonal Time-of-Flight Mass

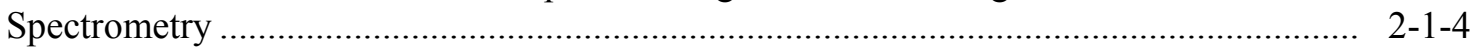

Transport Functions Dominate the SAR11 Metaproteome at Low Nutrient Extremes in the Sargasso Sea

MR Imaging of Apparent ${ }^{3} \mathrm{He}$ Gas Transport in Narrow Pipes and Rodent Airways ................ 2-1-6

A Solution NMR Investigation into the Early Events of Amelogenin Nanosphere SelfAssembly Initiated with Sodium Chloride or Calcium Chloride

Enhanced Detection of Low-Abundant Human Plasma Proteins using a Tandem IgY12-

SuperMix Immunoaffinity Separation Strategy

Global Systems-Level Analysis of Hfq and SmpB Deletion Mutants in Salmonella: Implications for Virulence and Global Protein Translation ............................................. 2-1-9

A Targeted Releasable Affinity Probe (TRAP) for in vivo Photo-Crosslinking ......................... 2-1-11

An Integrated Top-Down and Bottom-Up Strategy for Broadly Characterizing Protein Isoforms and Modifications ...................................................................................... 2-1-13

Platelet Proteome Changes Associated with Diabetes and during Platelet Storage for Transfusion.

Engineering an Ultra-Stable Affinity Reagent Based on Top7 ............................................ 2-1-15 
Identification of a Putative Protein Profile Associating with Tamoxifen Therapy

Resistance in Breast Cancer

Syndecan-1 Mediates the Coupling of Positively Charged Submicrometer Amorphous

Silica Particles with Actin Filaments across the Alveolar Epithelial Cell Membrane

Calmodulin Mediates DNA Repair Pathways Involving H2AX in Response to Low-Dose Radiation Exposure of RAW 264.7 Macrophages

Proteomic Investigation of the Time Responses of RAW 264.7 Macrophages to Infection with Salmonella enterica

Geochemistry/Biogeochemistry and Subsurface Science

Enhanced Remedial Amendment Delivery through Fluid Viscosity Modifications:

Experiments and Numerical Simulations

Kinetics of Reduction of Fe(III) Complexes by Outer Membrane Cytochromes MtrC and OmcA of Shewanella oneidensis MR-1

Antibody Recognition Force Microscopy Shows that Outer Membrane Cytochromes

OmcA and MtrC are Expressed on the Exterior Surface of Shewanella oneidensis

MR-1

Surface and Interfacial Properties of Nonaqueous-Phase Liquid Mixtures Released to the Subsurface at the Hanford Site

Electron Donor-Dependent Radionuclide Reduction and Nanoparticle Formation by Anaeromyxobacter dehalogenans Strain 2CP-C.

Reduction of $\mathrm{Hg}(\mathrm{II})$ to $\mathrm{Hg}(0)$ by Magnetite

Oxidative Dissolution Potential of Biogenic and Abiogenic TcO2 in Subsurface Sediments ...

Comparative Analysis of Urban Atmospheric Aerosol by Particle-Induced X-Ray Emission (PIXE), Proton Elastic Scattering Analysis (PESA), and Aerosol Mass Spectrometry (AMS)

Understanding How Surface Morphology and Hydrogen Dissolution Influence Ethylene Hydrogenation on Palladium

Vacancy-Assisted Diffusion of Alcoxy Species on Rutile $\mathrm{TiO}_{2}(110)$.

Self-Assembly of Cerium Oxide Nanostructures in Ice Molds

Intrinsic Diffusion of Hydrogen on Rutile $\mathrm{TiO}_{2}(110)$

Photoelectron Spectroscopic and Theoretical Study of $\mathrm{B}_{16}{ }^{-}$and $\mathrm{B}_{16}{ }^{2-}$ : An All-Boron Naphthalene

Imaging Consecutive Steps of $\mathrm{O}_{2}$ Reaction with Hydroxylated $\mathrm{TiO}_{2}(110)$ : Identification of $\mathrm{HO}_{2}$ and Terminal $\mathrm{OH}$ Intermediates

Two Pathways for Water Interaction with Oxygen Adatoms on $\mathrm{TiO}_{2}(110)$

Macrophage Responses to Silica Nanoparticles are Highly Conserved Across Particle

Sizes

Electronic Effects on the Surface Potential at the Vapor-Liquid Interface of Water

Self-Assembled $\mathrm{TiO}_{2}$-Graphene Hybrid Nanostructures for Enhanced Li-Ion Insertion $2-3-16$

Molecular Characterization of Nitrogen Containing Organic Compounds in Biomass Burning Aerosols Using High Resolution Mass Spectrometry.... 
Symmetry-Driven Spontaneous Self-Assembly of Nanoscale Ceria Building Blocks to Fractal Superoctahedra

Clay Nanoparticles-Supported Single-Molecule Fluorescence Spectroelectrochemistry

Line Intensities for the $\mathrm{v} 1, \mathrm{v} 3$, and $\mathrm{v} 1+\mathrm{v} 3$ Bands of ${ }^{34} \mathrm{SO}_{2}$

Co-Ordinatively Unsaturated $\mathrm{Al}^{3+}$ Centers as Binding Sites for Active Catalyst Phases on $\gamma-\mathrm{Al}_{2} \mathrm{O}_{3}$

Observation of a Remarkable Temperature Effect in the Hydrogen Bonding Structure and Dynamics of the $\mathrm{CN}^{-}\left(\mathrm{H}_{2} \mathrm{O}\right)$ Cluster

Single Wall Diesel Particulate Filter (DPF) Filtration Efficiency Studies using LaboratoryGenerated Particles

No Confinement Needed: Observation of a Metastable Hydrophobic Wetting Two-Layer Ice on Graphene

Defect-Enhanced Charge Transfer by Ion-Solid Interactions in SiC using Large-Scale $A b$ Initio Molecular Dynamics Simulations.

On the Phase Diagram of Water with Density Functional Theory Potentials: The Melting Temperature of Ice $\mathrm{I}_{\mathrm{h}}$ with the Perdew-Burke-Ernzerhof and Becke-Lee-Yang-Parr Functionals

In Situ Studies of Soft- and Reactive Landing of Mass-Selected Ions Using Infrared Reflection Absorption Spectroscopy....

An Isotropic Chemical Shift-Chemical Shift Anisotropic Correlation Experiment using Discrete Magic Angle Turning

Defining the Active Catalyst Structure and Reaction Pathways from Ab initio Molecular Dynamics and Operando XAFS: Dehydrogenation of Dimethylaminoborane by Rhodium Clusters

Low-Energy Networks of the T-Cage $\left(\mathrm{H}_{2} \mathrm{O}\right)_{24}$ Cluster and Their Use in Constructing Periodic Unit Cells of the Structure I (sI) Hydrate Lattice $2-3-36$

Fiscal Year 2009 User Projects

Fiscal Year 2009 Publications

Journal Articles

Book Chapters 



\section{Overview}

This Fiscal Year 2009 Annual Report describes the research and accomplishments of staff and scientific users of EMSL - the Environmental Molecular Sciences Laboratory, located in Richland, Washington.

\section{Mission}

EMSL, a national scientific user facility at Pacific Northwest National Laboratory, provides integrated experimental and computational resources for discovery and technological innovation in the environmental molecular sciences to support the needs of DOE and the nation.

\section{EMSL Resources}

EMSL is a national scientific user facility available to researchers worldwide from academia, industry, and national laboratories. EMSL scientific users pursue the understanding of molecular systems essential to scientific breakthroughs and discoveries for a broad set of DOE missions in energy, environment, climate, and national security. Staff at EMSL develop and maintain extensive advanced research and development capabilities that are used to generate new scientific knowledge. EMSL delivers substantial value to its scientific users by understanding their needs, creating responsive new ideas and capabilities, and delivering exceptional results - all achieved through the expertise of staff, demonstrated excellence in management and laboratory operations, and high-value partnerships with its scientific users.

The operating budget for EMSL is provided by DOE Office of Biological and Environmental Research (BER), while the research conducted within the facility is beneficial to the DOE Office of Science and many funding agencies, including other offices within DOE, the National Institutes of

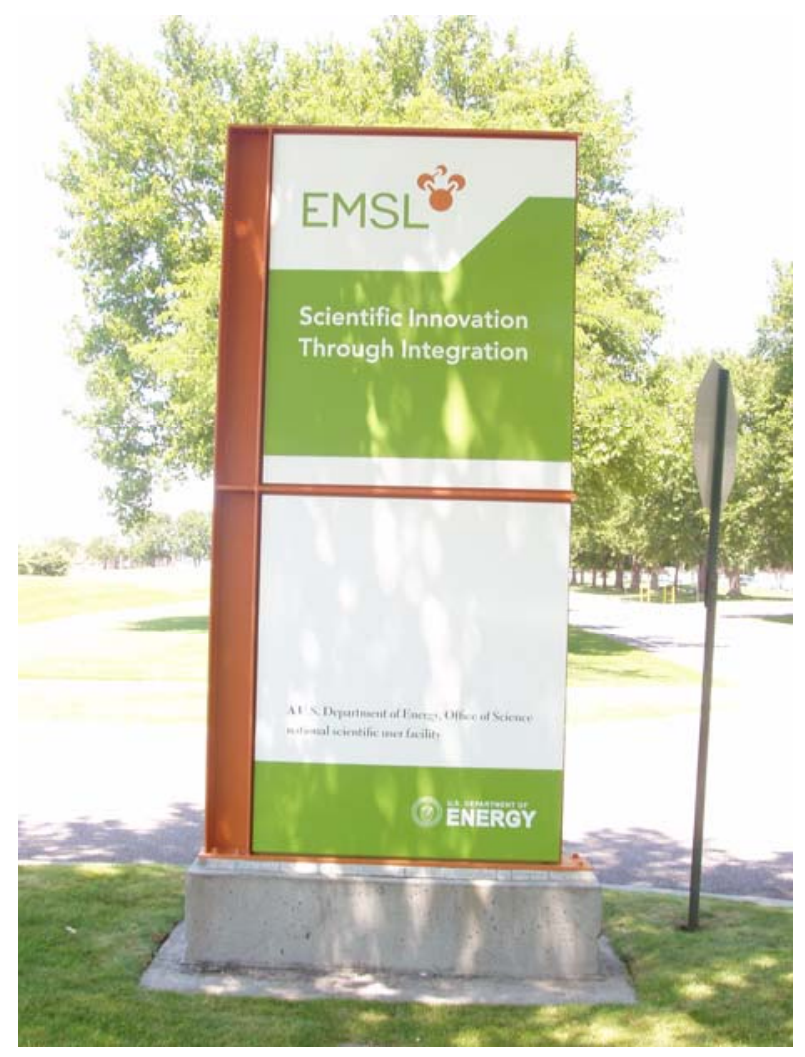

EMSL is a DOE national scientific user facility located at Pacific Northwest National Laboratory in Richland, Washington. Health, the National Science Foundation, and the Department of Defense.

Since beginning operations in October 1997, EMSL has provided advanced and one-of-a-kind integrated experimental and computational resources to scientists engaged in fundamental research in the physical, chemical, and biological processes that underpin environmental remediation and other important scientific 
issues facing DOE and the nation. In addition to physical resources, EMSL provides unprecedented technical support and expertise, providing its scientific users a highly efficient and focused resource that enhances their work.

\section{Organization}

In Fiscal Year 2009, EMSL was managed by a Director, an Associate Director for Scientific Resources, an interim Associate Director for Molecular Science Computing, a Chief Operations Officer, a Chief Scientist and Lead Scientists, a Chief Technology Officer, and Capability Stewards in the areas of Cell Isolation and Analysis, Deposition and Microfabrication, Mass Spectrometry, Microscopy, Molecular Science Computing, NMR and EPR, Spectroscopy and Diffraction, and Subsurface Flow and Transport.

In addition, two advisory committees - the Science Advisory Committee (SAC) and the User Advisory Committee (UAC) - provide advice to the EMSL Director on longer-term scientific direction and shortterm, user-related issues, respectively.

\section{Science Advisory Committee}

The independent SAC provides objective advice on long-term science direction to the EMSL Director related to topics such as:

- EMSL's focus on national priorities and science challenges

- strategy for accomplishing EMSL's vision

- potential opportunities for transferring science to applications

- stewardship.

The SAC is comprised of distinguished scientists from academia, national laboratories, and research institutions across the United States who provide expertise in EMSL's major capability areas of biology, environmental science, molecular science, and theory. The committee reports to the Director, PNNL. Responsibilities and activities of the SAC include:

- providing advice on scientific direction for the EMSL user program

- recommending appropriate balance of instrument time for Grand Challenges, general scientific users, and capability development activities

- assisting EMSL with formulating policies related to facility scientific output, scientific impact of the EMSL user program, user access, and other issues, as needed

- performing periodic reviews of scientific impact from the various elements of the EMSL scientific user program.

The committee meets annually at EMSL and the 15 members serve staggered 4-year terms. The current members of the SAC are as follows:

- Len Spicer, Duke University (Chair)

- Mark A. Barteau, University of Delaware 
- Gordon E. Brown, Jr., Stanford University

- Charles T. Campbell, University of Washington

- Marvin Cassman, Consultant

- Gregory Choppin, Florida State University

- Thom Dunning, University of Illinois at Urbana-Champaign

- Ian Farnan, Cambridge University

- Barbara J. Finlayson-Pitts, University of California, Irvine

- George W. Flynn, Columbia University

- Gary Hieftje, Indiana University

- Ian Jardine, Thermo Fisher Scientific, Inc.

- Samuel Kaplan, University of Texas-Houston Medical Center

- Julia Rice, IBM Almaden Research Center

- James M. Tiedje, Michigan State University

- Mary F. Wheeler, University of Texas at Austin.

\section{User Advisory Committee}

The UAC is an independent body of experts from academia, industry, and the national laboratory system that is charged with providing objective, timely advice and recommendations to EMSL leadership. The Committee reports directly to the EMSL Director and serves as the official voice of EMSL's scientific user community in its interactions with EMSL management.

The responsibilities of the User Advisory Committee include:

- providing a clear channel for the exchange of information and advice between EMSL scientific users and management

- providing a formal vehicle for EMSL scientific users to transmit concerns and recommendations to the EMSL Director

- designing and overseeing the EMSL User Meetings

- providing advice and recommendations to the EMSL Director on how to facilitate the effective use of EMSL

- nominating active scientific users for future membership to the committee.

The UAC members are:

- Kerry Hipps, Washington State University (Chair Elect)

- Valérie Copié, Montana State University

- Mary Gilles, Lawrence Berkeley National Laboratory 
- Vicki H. Grassian, University of Iowa

- Anne M. Johansen, Central Washington University

- Sarah C. Larsen, University of Iowa

- Brian Lower, The Ohio State University

- Karl T. Mueller, Pennsylvania State University

- Lisa Porter, Carnegie Mellon University

- Sudipta Seal, University of Central Florida

- Paul G. Tratnyek, Oregon Health \& Science University

- Angela K. Wilson, University of North Texas

- Theresa L. Windus, Iowa State University (Chair).

\section{Science Themes}

EMSL science themes are areas where EMSL can make significant scientific contributions. Current science themes build upon and expand the historical scientific expertise of EMSL. They were evaluated against key criteria including DOE and national relevance, contribution to impactful environmental molecular science, strong national science and technology programs, and the ability to grow a vibrant national user program, and they were selected with broad input and support from the scientific community and EMSL advisory committees. As scientific advances are made, the nature and elements of EMSL's science themes will evolve to reflect both scientific gains and changing societal needs. New areas will be explored and evaluated during annual business planning. EMSL's three science themes are outlined in more detailed with relevant highlights given in each theme in Section 2, are (1) Biological Interactions and Dynamics; (2) Geochemistry/Biogeochemistry and Subsurface Science; and (3) Science of Interfacial Phenomena.

\section{Proposals and Scientific User Access Modes}

Users are encouraged to submit proposals in response to specific calls for proposals at defined times of the year, or in response to the Open Call at any time during the year.

\section{Calls for Proposal Focus}

To maximize the impact of EMSL research, the following proposal types are available for users. Preference is given to user proposals submitted in response to periodic calls for proposals, although user proposals requesting one year of access or less may be submitted at any time during the year. Although a limited amount of access may be available for proprietary research, most research conducted at EMSL is nonproprietary, with results disseminated to the scientific community through publications in the open literature or conference presentations and papers. Authors of nonproprietary proposals may retain rights to intellectual property (IP) resulting from the use of EMSL, but the government is granted a nonexclusive license to use the IP. All proposals are submitted through EMSL's User Portal and undergo management and peer reviews. 


\section{Periodic Calls}

EMSL solicits user proposals in response to periodically published calls for proposals. This enables us to collectively review, prioritize, and accept user proposals that are competing for access to limited resources. EMSL's calls for proposals emphasize research opportunities that will advance EMSL's Science Themes or will utilize EMSL's unique research tools for novel applications. In addition, details from previous calls for proposals provide insight about the how the research focus at EMSL has evolved over time.

Science Theme Users are particularly encouraged to submit proposals that fit into major areas of current and growing user activity associated with scientific challenges that address topics of societal importance. Science Theme proposals are valid for one year and may be extended twice for a total period of three years. Some EMSL resources may be principally dedicated to Science Theme proposals.

Computationally Intensive Research in Environmental Science Computationally Intensive Research (CIR) proposals focus on one or more of the topics highlighted in the published call as well as on research of an algorithmic nature that might have a broad application to environmental research. CIRs are expected to utilize EMSL's computing resources predominantly for large-scale parallel calculations that scale efficiently with both the number of processors and size of problem. Project proposals are expected to comprise multiple investigators working collaboratively as a team. A collaborative team approach provides an excellent mechanism to engage the expertise of researchers from a variety of institutions to be focused on the basic and applied research projects needed to solve the environmental problems of interest to DOE and the nation. Computer allocations for CIRs are valid for one year and may be extended twice for a total access of three years, with the computer allocation appropriate for the scope of research to be performed.

Capabilities-Based Capabilities-based proposals are sought when capabilities are not fully subscribed or when new capabilities are brought online. Although the details of access can vary according to the Call, capability-based proposals are typically awarded access for one year with one extension possible, for a total access of two years.

\section{General}

Use of existing EMSL resources typically falls into the general use category. General use proposals may be submitted to EMSL at any time throughout the year, and they are evaluated by peer review before research can begin. Proposals may be from individuals or groups who need access to EMSL to carry out their research, using existing equipment at EMSL. The scope of a general use proposal can vary from a single experiment proposal to full project proposal (valid for multiple visits and substantial access to a range of equipment). Individual and group proposals, including collaborative proposals with EMSL staff, are encouraged.

\section{Rapid Access}

In limited cases, users may need access to EMSL capabilities where rapid turnaround of data is required for a specific deadline (e.g., response to requested data for finalizing thesis work or paper publication, or preliminary data needed for proposal preparation). If approved, a rapid access proposal will be valid for no more than one month of EMSL use. To be considered under this category, proposal descriptions 
must clearly justify why rapid access is needed and provide the working deadline. All proposals will undergo peer review, although work can be started as soon as management and safety approvals are completed and subject to instrument and resource availability.

\section{Proprietary Research}

EMSL facilities can be used for proprietary research. DOE requires that such work pay full-cost recovery of the facilities used, which includes, but is not limited to, labor, equipment usage, consumables, materials, and EMSL staff travel. User proposals containing proprietary information will be reviewed under a restricted access process to maintain confidentiality.

\section{Partner}

Partner proposals are submitted by individuals or groups who wish to partner with EMSL staff to enhance an existing capability or develop and build unique new capabilities that enhance EMSL's user program. Capability development efforts that support environmental molecular sciences and which utilize collaborative multidisciplinary teams, pooled or leveraged resources, unique operating environments, or other resources which may be beyond those available to individual researchers or teams are encouraged. Partner proposals are intended to leverage expertise, capability, and resources that maximize impact for EMSL, the partner, and future users. In return for co-development, EMSL partner users may have priority access to the new capability for a negotiated and specified period (subject to EMSL Advisory Committees review and approval). Proposals may be in response to a specific call or submitted at any time. The award and timing of EMSL Partner Projects are contingent upon EMSL strategic needs and the availability of EMSL resources.

A two-page Letter of Intent (LOI) is used to initiate a dialog with EMSL's Chief Technology Officer on suitability, interest, and strategic need for the capability. A Partner user is encouraged to work with appropriate EMSL Capability Stewards or other technical contacts in preparing the Letter of Intent, which should include initial discussion of need, approach, resources, partner contributions, impact, and proposed team.

A Letter of Intent may be submitted by a partner user at any time to emsl@pnl.gov, and will be reviewed by a panel consisting of the Chief Technology Officer, the Chief Science Officer, the Associate Director for Scientific Resources, and the Lead Scientists. Review criteria will include strategic alignment, user/scientific impact and need, and resource and time requirements. Interaction, deliberation, and refinement of concepts with the Committee and/or EMSL staff should be expected during the LOI review process. Upon review and approval, the Chief Technology Officer or delegate will contact the partner user and request a full proposal, along with specific needs, considerations, or contacts to be addressed.

Full Partner Proposals (6-page maximum) are to be submitted to the EMSL User Portal, and will detail specific needs, considerations, or contacts to be addressed. Partner users are encouraged to work with EMSL staff in refining and developing a full proposal that meets identified EMSL capability needs and is consistent with EMSL strategy, science themes, and technology thrusts.

Full proposals will be reviewed by (selected) members of EMSL's advisory committees (Scientific Advisory Committee, User Advisory Committee) and an EMSL panel consisting of the Chief Technology Officer, the Chief Science Officer, the Associate Director for Scientific Resources, selected Lead 
Scientists, and other ad-hoc members as may be required for technical evaluation. Review criteria will include strategic alignment, user/scientific impact and need, and resource and time requirements.

Additionally, all meritorious proposals will be reviewed by the EMSL Chief Operations Officer as part of the approval process. The Chief Technology Officer will be responsible for communicating final approval decisions to the proposal authors.

\section{Calls for Proposals in Fiscal Year 2009}

In Fiscal Year 2009, EMSL received 308 user proposals, including 20 requests for Computationally Intensive Research proposals and 7 partner proposals based on approved Letters of Intent. Of the 308 proposals, 54 involved teams that had not previously used the facility. 


\section{EMSL Highlights}

\section{Major EMSL Activities}

Chinook is accepted. EMSL's new supercomputer, Chinook, achieved final acceptance on March 20, 2009. Chinook is a \$20M system with peak performance of 164 teraflops, nearly 15 times faster than EMSL's previous 11.2 teraflop system named MPP2. Chinook can perform more 160 trillion calculations per second, ranking it in the top 20 fastest computers in the world. Speed is not its focus though: Chinook's designers made sure the supercomputer can handle the kinds of complex scientific problems the researchers who use it tackle. Researchers studying complicated worldly questions - from climate

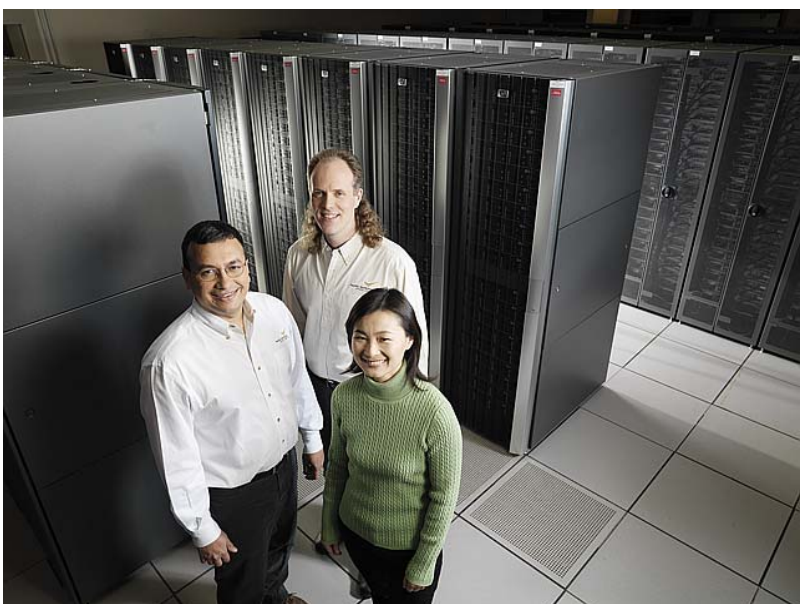

EMSL's Chinook received final acceptance on March 20, 2009. scientists who are trying to reproduce the tiniest particles in the atmosphere to chemists watching how atoms tug at each other in a molecule - need a different kind of supercomputer than physicists studying the big bang.

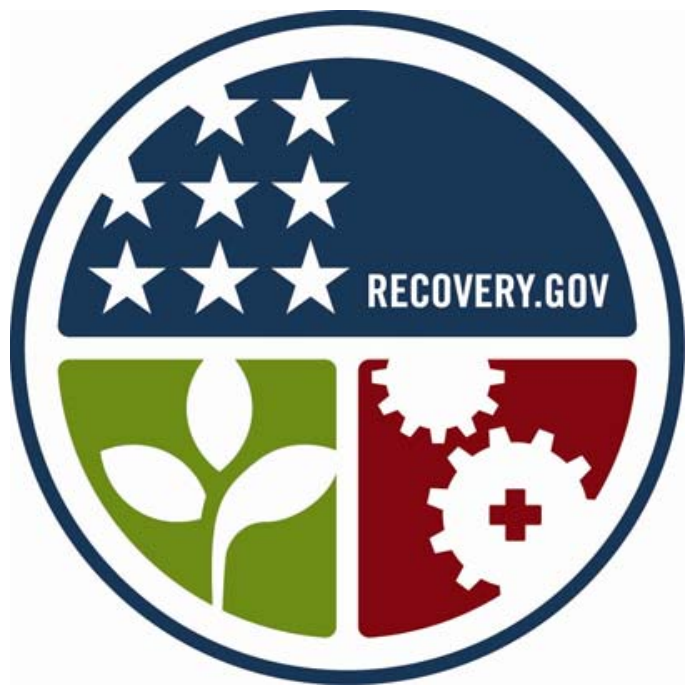

EMSL has received $\$ 60$ million in Recovery Act funds for accelerating existing plans to enhance its user program.
EMSL receives Recovery Act funding. DOE is investing in Pacific Northwest National Laboratory through the American Recovery and Reinvestment Act. EMSL has been awarded $\$ 60 \mathrm{M}$. These funds are allowing EMSL to accelerate existing plans to enhance its user program, developing and deploying systems and capabilities that maintain EMSL's position as a state-of-the-art user facility. This funding will enable high-end scientific capabilities, including nuclear magnetic resonance spectrometry, mass spectrometry, and high-powered microscopy. All instruments will be received by EMSL no later than December 31, 2010. 


\section{Energy Secretary Chu visits EMSL. On \\ August 10, 2009, Energy \\ Secretary Steven Chu visited Pacific Northwest National Laboratory and toured EMSL's NMR capabilities, where he learned about the carbon sequestration and catalysis work that EMSL enables. The visit was followed by arrival of Deputy Energy Secretary Daniel Poneman on August 18.}

EMSL's supercomputer featured in SciDAC Review. EMSL's new supercomputer, Chinook, was featured in the Summer 2009 issue of SciDAC Review (http://www.scidacreview.org/0903/html/hardware.html).

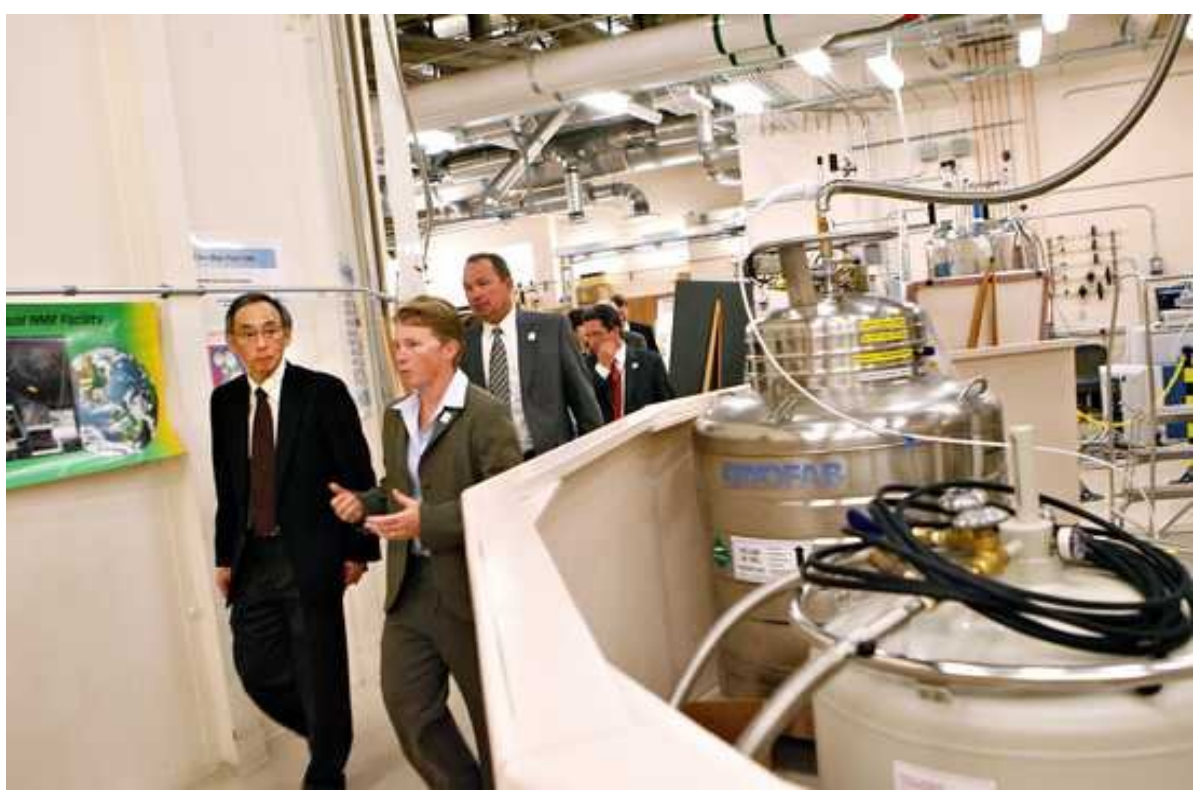

EMSL Director Allison Campbell (right) explains EMSL's NMR capabilities to Energy Secretary Steven Chu on a visit to PNNL on August 10. Following is PNNL Director Mike Kluse. Photo courtesy of the Tri-City Herald. 


\section{EMSL Staff and Scientific User Highlights}

In 2009, EMSL staff and scientific users accomplished the following:

Baer receives AAAS, AVS, editorial honors. Don Baer, EMSL's Lead Scientist for Interfacial Chemistry, was named Fellow of the American Association for the Advancement of Science. At EMSL, Baer specializes in the use of spectroscopy and other advanced techniques to reveal the behavior of atoms and molecules at or near the surfaces of materials. AAAS honored him "for research and capability development that significantly advance molecular-level understanding of environmentally important interfacial processes relevant to nanoparticle reactivity, mineral dissolution and stress corrosion cracking." Baer joined 485 other high-caliber researchers who were elected as Fellows in November, including EMSL users Cindy Bruckner-Lea, Michel Dupuis, Chuck Peden, and Yong Wang. He was honored with his fellow recipients at the AAAS Annual Meeting to be held in February in Chicago.

Baer also received the 2009 Albert Nerken Award from the AVS. The award recognizes individuals who have made

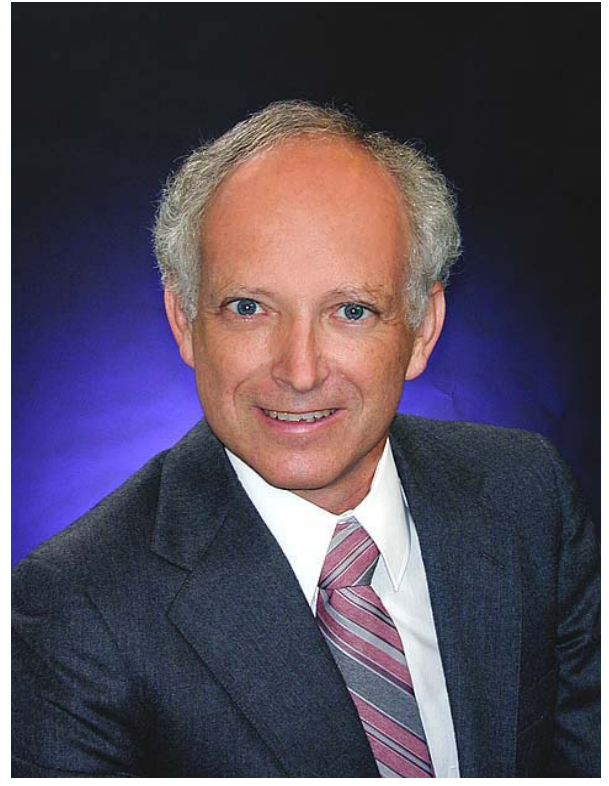

Don Baer outstanding contributions to the solution of technological problems in areas of interest to AVS. Baer was cited "for seminal contributions towards advancing the application of surface-sensitive techniques to understand environmentally important materials and interfacial processes." Baer largely attributes the award to Pacific Northwest National Laboratory's rich history of multidisciplinary, team-oriented research that enables significant progress on complex problems. "Although the award is presented to an individual, much of my research has involved participating on and leading teams," he says. "These teams have made important advances in understanding stress corrosion cracking, oxide and mineral surface chemistry, and dynamic behaviors of nanoparticles, as well as in developing and applying interfacial tools in EMSL that facilitate such advances." The award was established in 1984 by Veeco Instruments, Inc., in recognition of Albert Nerken for his role as a founding member of AVS, his early work in the area of high vacuum and leak detection, and his contributions to the commercial development of that instrumentation. Presentation of the award took place at the AVS International Symposium, in San Jose, California, in November.

Finally, Baer was selected to serve as the Reviews Editor for Surface and Interface Analysis. This international, refereed journal is devoted to publishing papers on developing and applying techniques for characterizing surfaces, interfaces, and thin films. As the Reviews Editor, Baer will work with worldleading scientists to obtain articles on topics of interest to the journal's audience of materials scientists, physicists, physical chemists, and others. In addition to traditional review articles, he will encourage leading scientists to prepare perspectives on current research directions and technology advances and tutorial articles. Baer is internationally known for applying surface analysis methods to examine the reactive properties of oxides and mineral surfaces, determining behaviors of nanostructured materials, and 
understanding corrosion processes. He specializes in using surface sensitive techniques to study surface interphase reactions and materials surface chemistry.

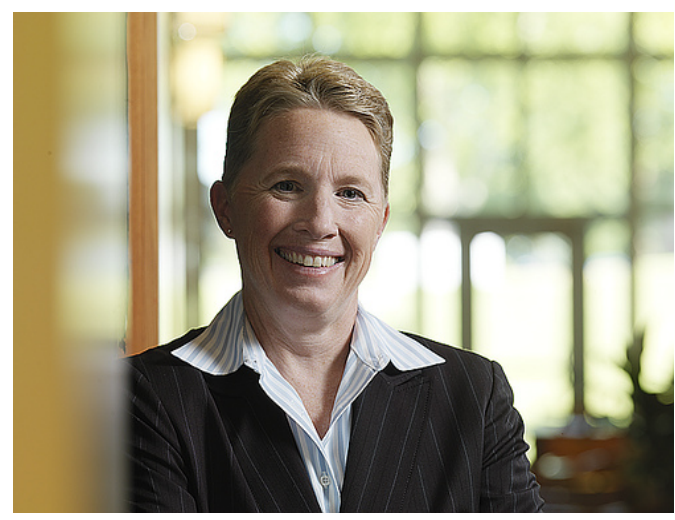

Campbell
Campbell testifies before House Committee. Allison A. Campbell, Director of EMSL, testified on September 10, $\underline{2009}$, before the Subcommittee on Energy and Environment of the House Committee on Science and Technology about how EMSL's unique facilities and instruments help DOE meet its mission goals through biological research. See the PNNL news release and video at http://www.pnl.gov/news/release.aspx?id=400.

\section{Zhang named to journal's advisory editorial} board, invited to serve guest professorship. Congratulations to EMSL researcher Yanwen Zhang, who was invited to serve a 3-year term on the advisory editorial board of Nuclear Instruments \& Methods in Physics Research, Section $B$. This journal, published by Elsevier, covers all aspects of the interaction of energetic beams, such as ion, electron, and photon beams, with solids. Related topics, such as applying ion beam analysis to biological, archaeological and geological samples, are covered. Notable conferences publish their proceedings in this peerreviewed journal.

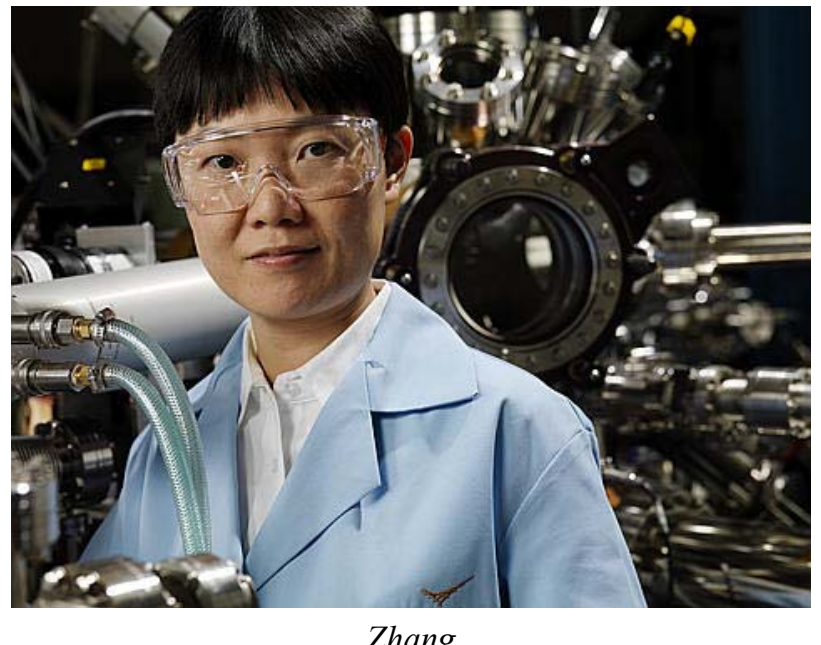

Zhang

Zhang has accepted an invitation to serve a two-year term as Guest Professor of Peking University. In this capacity, she will monitor Ph.D. students at EMSL who are mainly supported by the Chinese government and help strengthen materials research at the university, until March 2011. Zhang will visit Peking University approximately twice yearly, and the university professors, researchers and students will also visit EMSL as part of the collaboration. The invitation was extended by Professor J. F. Zhou, the President of Peking University. 
Wiley invited to join National Research Council Review Panel. Steven Wiley, EMSL Lead Scientist for Biology, was selected to serve as a reviewer on the 2009 National Research Council Research Associateship Programs. The programs fund postdoctoral fellows, senior scientists and engineers, and faculty to work on research problems of their choice in federal laboratories. The Council receives around 800 applications every year for these programs. The NRC selects about 300 of these applications for funding based on a competitive process that includes a review by one of five NRC panels. One of the five panels is the Life Sciences panel, where Wiley will serve. On this panel, he will evaluate the impact and the approach of the research proposed in the applications four times a year. Also, he will discuss his recommendations with his colleagues on the panel, working to promote only the best possible research. Wiley will serve on the panel for three years. He was chosen because of his scientific expertise in molecular and systems biology, such as the research into large-scale protein-protein interactions and cell signaling networks. In

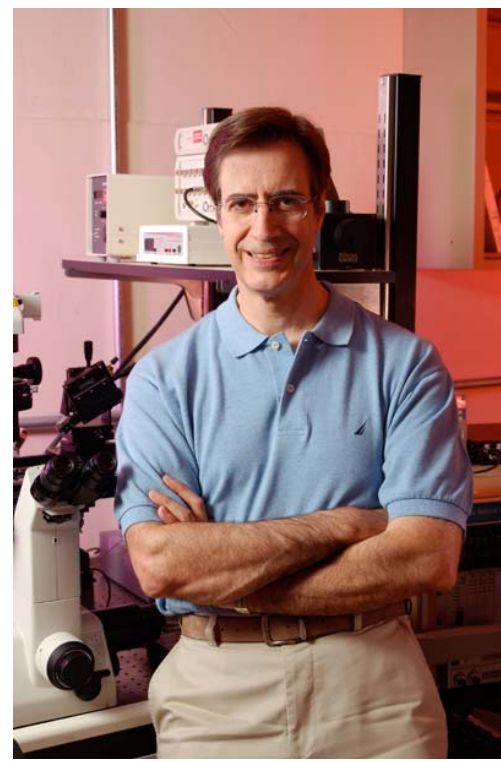

Wiley addition, he is an experienced leader, currently working as the steward for EMSL's Biological Interactions and Dynamics science theme and as Director of Pacific Northwest National Laboratory's Biomolecular Systems Initiative. Further, he has experience with advisory committees, including the National Institutes of Health and Burroughs Wellcome Fund.

\section{Koppenaal invited to serve on journal international advisory board,} elected division chair of ACS. David Koppenaal, EMSL's Chief Technology Officer, was invited to serve a 3-year term on the International Advisory Board for the new journal Metallomics: Integrated Biometal Science. The journal is a Royal Society of Chemistry publication that will be launched in January 2009. Metallomics is the integrated study of metals and metal species and their interactions, transformations, and functions in biological systems. The subject is receiving attention as a new frontier in the investigation of trace elements in biology. Metallomics is expected to develop as an interdisciplinary science complementary to metabolomics and proteomics. At EMSL, Koppenaal leads numerous biological studies. His research is primarily focused on characterizing inorganic compounds and isotopes with innovative mass spectrometry and other analytical techniques. For example, Koppenaal and his colleagues at Pacific Northwest National Laboratory pioneered the application of

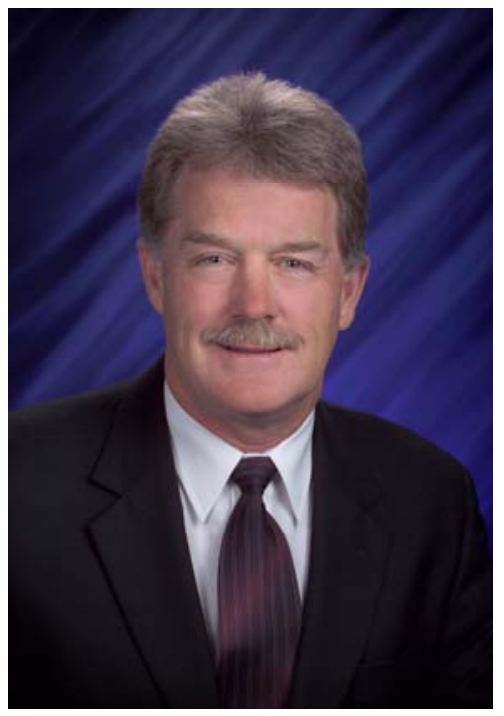

Koppenaal inductively coupled plasma/mass spectrometry as a powerful and relevant radioanalytical tool and demonstrated its use for radioactive waste characterization, ultra-trace nuclear forensics, and, most recently, metallomics applications. He is also responsible for leading the development of world-leading capabilities for EMSL, such as a current collaboration with the National High-Field Magnetic Laboratory to develop a 21-Tesla magnet that will lead to the highest resolving power and mass accuracy possible in mass spectrometry. His more than 75 publications include several invited review articles, and he is a frequent keynote and plenary lecturer at major conferences. Koppenaal recently completed a 7-year term as Editorial Board member for the RSC publication Journal of 
Analytical and Atomic Spectrometry. Koppenaal is a fellow of the American Association for the Advancement of Science and the Royal Society of Chemistry. He is a member of the American Chemistry Society and the American Society for Mass Spectrometry.

Koppenaal was also elected to a national society-level position for the American Chemical Society. He will serve as Chair-Elect for the Analytical Chemistry Division of the society starting October 1, 2009, and succeed to Program Chair in 2010 and Chair in 2011. The Analytical Chemistry Division is one of the largest of 34 divisions of the ACS, the world's largest scientific society. The division has approximately 9500 members, and its vision is "Improving people's lives through the transforming power of analytical chemistry and promoting the community of analytical scientists."

Qian named Rising Young Star. Wei-Jun Qian, an EMSL user from Pacific Northwest National Laboratory, was named one of the 30 rising young stars of science by Genome Technology magazine in its third annual "Tomorrow's PIs" special edition. The magazine offers readers a chance to see large-scale biological research through the eyes of some of the best and the brightest young scientists who are poised to make significant contributions in advancing the scientific frontiers. Qian was selected for this honor because of his work in developing and applying innovative techniques that have enabled large-scale, quantitative investigations of proteins in challenging clinical samples. Currently, he is developing and applying novel mass spectrometrybased approaches to measure changes in proteins from mammalian cells, tissues, and biofluids. His work is helping other scientists better understand cell signaling and discover mechanistic or diagnostic protein biomarkers for human diseases. The magazine's interview with Qian, titled "Step by Step, a Better Mass Spec," appeared in the December 2008/January 2009 issue.

\section{Smith named PNNL Inventor of the Year, Editor in Chief of} Journal of Proteomics \& Bioinformatics. EMSL user Richard D. Smith (Pacific Northwest National Laboratory) was named the PNNL Inventor of the Year for Fiscal Year 2008. At EMSL, Smith has taken part in developing and applying advanced analytical methods and instrumentation, with an emphasis on high-resolution separations and mass spectrometry, to environmental, biological and biomedical research. Current applications include studies related to carbon sequestration, as well as the development of biomarkers for breast and brain cancer, neurological diseases, and diabetes and kidney diseases, among others. In addition, Smith has accepted an invitation to become Editor-in Chief of the Journal of Proteomics \& Bioinformatics. Smith sets the direction for the open-access journal and gives final approval for each issue. Founded in 2008, the Journal of Proteomics \& Bioinformatics
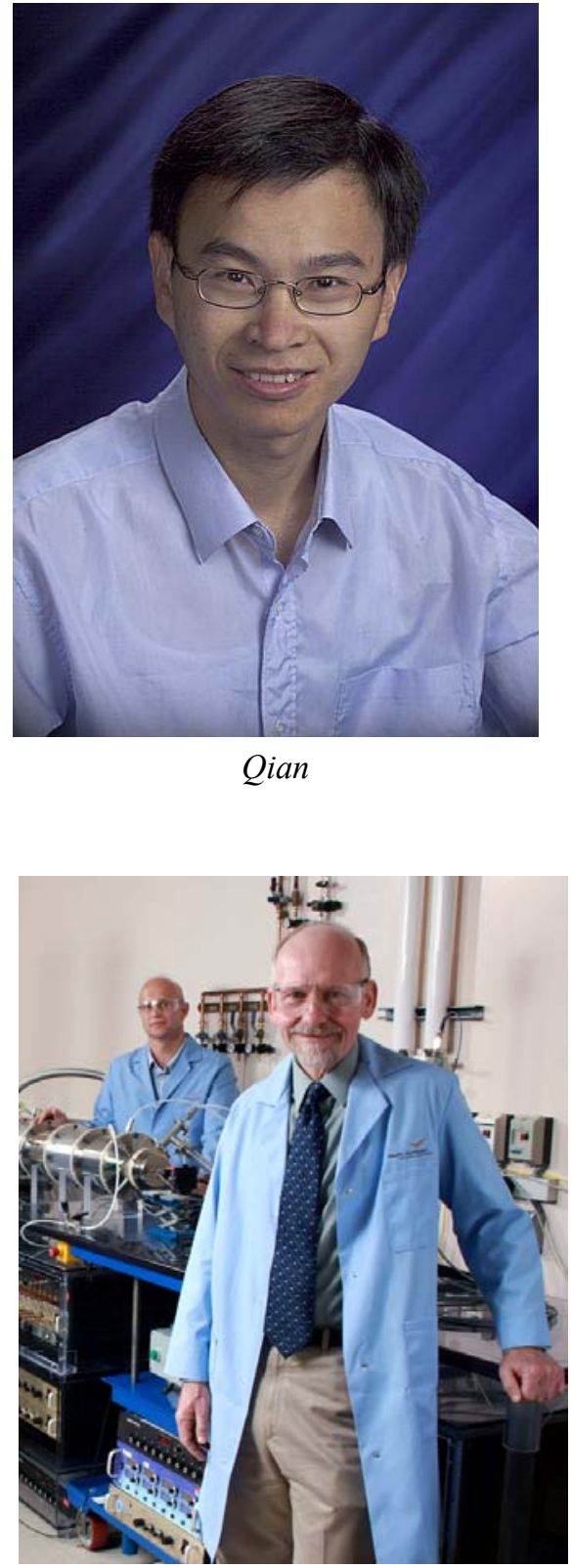

Smith 
strives to provide rapid review and publishing of research in the proteomics and bioinformatics fields. The journal disseminates its articles freely for research, teaching and reference purposes.

Rosso selected as Virginia Tech Outstanding Alumnus. EMSL user Kevin Rosso (Pacific Northwest National Laboratory) was honored by Virginia Tech for his outstanding research and contributions to the scientific community. Each year the university's alumni association selects, from each academic college, a gifted person who has graduated in the past decade. Rosso received the award from the College of Science, where he earned a doctoral degree in geochemistry in 1998. Rosso leads internationally recognized research in environmental spectroscopy and biogeochemistry. He is an expert in mineral-water interface geochemistry, combining scanning probe microscopy and ab initio molecular modeling. He is known for his insightful models of electron transfer kinetics in environmental systems. His recent studies illuminate the relationship between bacteria and environmental metals, such as iron and uranium. Rosso has written or co-written more than 80 peer-reviewed journal articles, including a recent article

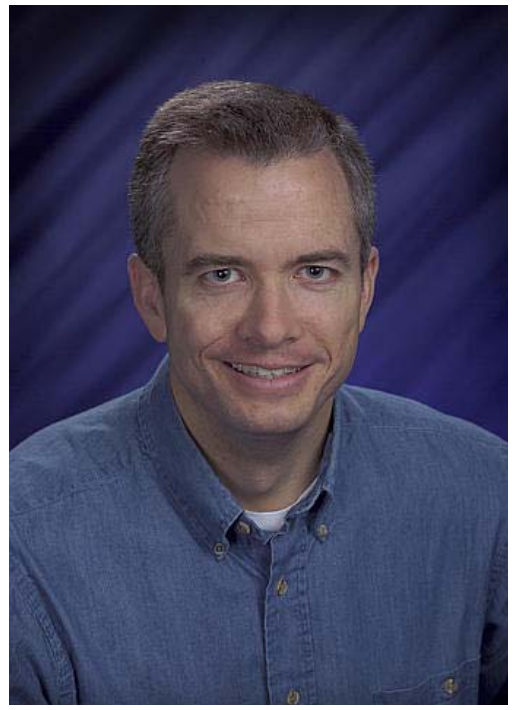

Rosso in Science. He is frequently invited to lecture at universities and symposia, in the United States and abroad. In addition, he is a life fellow of the Mineralogical Society of America, and he served for two years as an associate editor for American Mineralogist. He is a member of the American Chemical Society and has organized topical symposia for the society's national meetings.

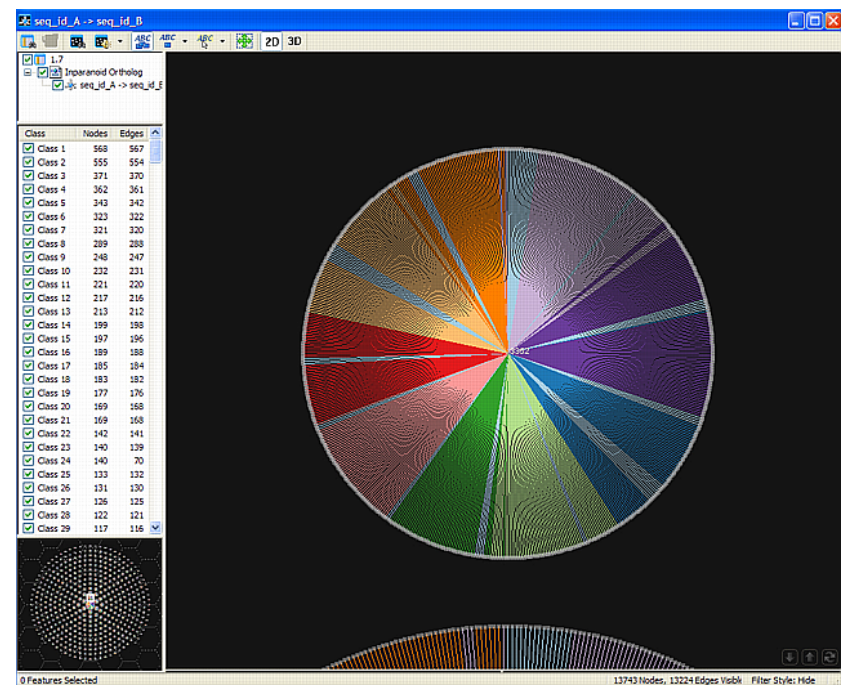

A computer program developed by EMSL users with help of EMSL computational resources shows that a graphical cluster compares 42,000 unknown proteins from 10 species of Shewanella against a single known protein at the center of the pie. The user has zoomed in on this cluster from the panoply of clusters at the bottom left of the screen.

\section{EMSL users earn top honors at}

Supercomputing 08. DNA sequencing is easier than ever, but the amount of data to be analyzed is piling up. An award-winning computer program now shows that genome sequence analysis can be made interactive and intuitive, helping researchers find hidden relationships in massive amounts of data.

Researchers from the Pacific Northwest National Laboratory captured "Best Overall" for their entry at the Supercomputing '08 High Performance Computing Analytics Challenge in Austin, Texas, on November 20.

In the competition, scientists were judged on solving real world problems using comprehensive computational approaches, large data sets, and high-end visualization technology to display results - which means it had to look good and be easy to use. 
PNNL's Chris Oehmen led a multidisciplinary team composed of Scott Dowson, Chandrika Sivaramakrishnan, Justin Almquist, Lee Ann McCue, Bobbie-Jo Webb-Robertson, and Jason McDermott to the win. Resources the team used included computing capabilities housed in EMSL to develop the interactive program.

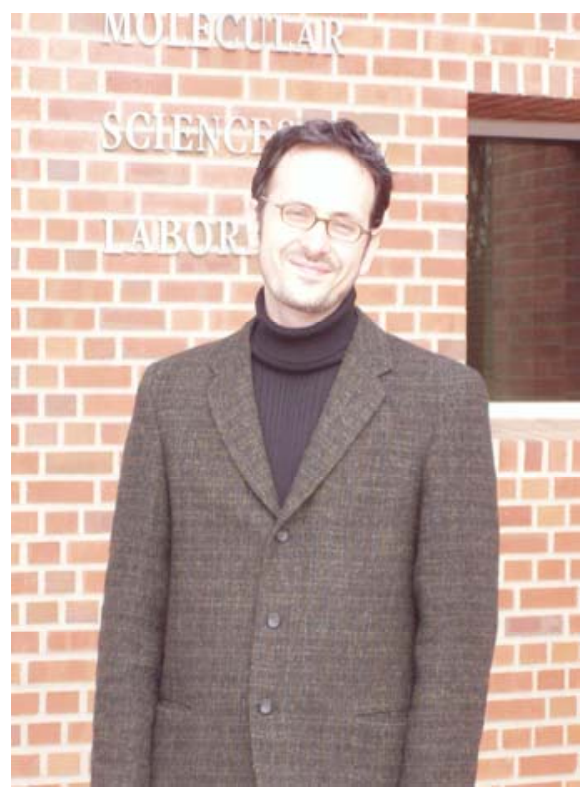

Raff

Raff receives prestigious NSF award. Jonathan Raff, an EMSL user from the University of California Irvine, was awarded a prestigious American Competitiveness in Chemistry Postdoctoral Fellowship from the National Science Foundation. The award is designed to support post-doctoral associates in chemistry and seeks to build ties between academia and industry/the national laboratory system while also involving beginning scientists in efforts to broaden participation in chemistry. Raff is one of four researchers nationwide who received the award, which is in its inaugural year. A research associate at the university's Environmental Molecular Sciences Institute, AirUCI - or Atmospheric Integrated Research for Understanding Chemistry at Interfaces, he received the award in the amount of $\$ 200,000$ from the NSF's Division of Chemistry for his proposal to study the heterogeneous reactions of nitrogen oxides under atmospherically relevant conditions. Nitrous acidwhich results from the heterogeneous reaction of nitrogen dioxide and water - is the most important daytime source of reactive hydroxyl free radicals, an important oxidant that removes pollutants and drives important radical reaction pathways in the atmosphere. However, there is currently very little understanding about how nitrous acid forms on surfaces, so Raff's goal is to use EMSL's surface science instruments, such as infrared spectroscopy, X-ray photoelectron spectroscopy, time-of-flight secondary ion mass spectrometry, and Auger electron spectroscopy to help identify its precursors on surfaces and gain insight and understanding of how the chemistry leading to nitrogen dioxide adsorption and photochemical nitrous acid formation affects the composition of aerosol and thinlayer film surfaces.

\section{Bill Weber named Distinguished Alumnus by University of Wisconsin Oshkosh. EMSL user Bill Weber (Pacific Northwest National Laboratory) was selected for the Distinguished Alumni Award, the highest University of Wisconsin Oshkosh alumni honor given. Weber and eight others will be honored for their professional and civic achievements during homecoming weekend, October 16-17, 2009. Weber is an internationally recognized expert in defects and radiation effects in materials. His research, often done at EMSL, is essential for advanced electronic devices that operate in extreme conditions, developing radiation-tolerant materials for nuclear power, as well as for space exploration and research.}

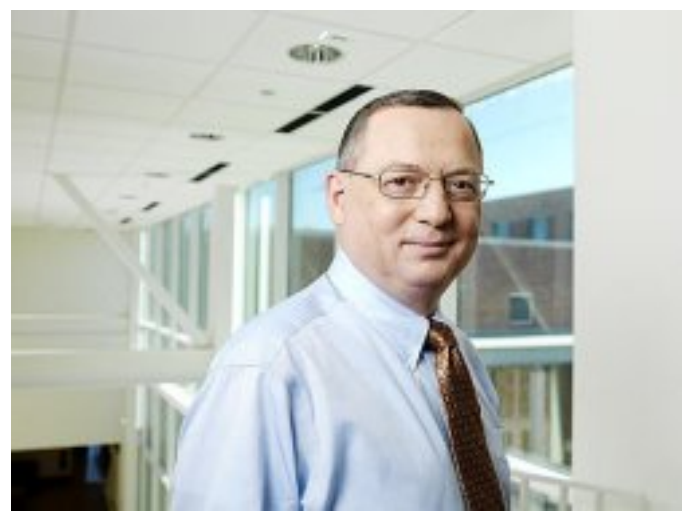

Weber 
Julia Laskin honored with special issue of Prestigious Spectroscopy

Journal. EMSL user Julia Laskin (Pacific Northwest National

Laboratory) was honored with a special issue of the Journal of the American Society of Mass Spectrometry in June 2009, now available online. The issue, containing invited articles by several experts, recognizes the 2008 Biemann Medal for her early career work in mass spectrometry. In the scientific community, Laskin is internationally known for her work on gas-phase ion chemistry and mass spectrometry of large, complex molecules. With her colleagues from around the world, she is designing techniques to better characterize complex molecules in biological samples, atmospheric aerosols, and biofuels, work critical for a secure energy future.

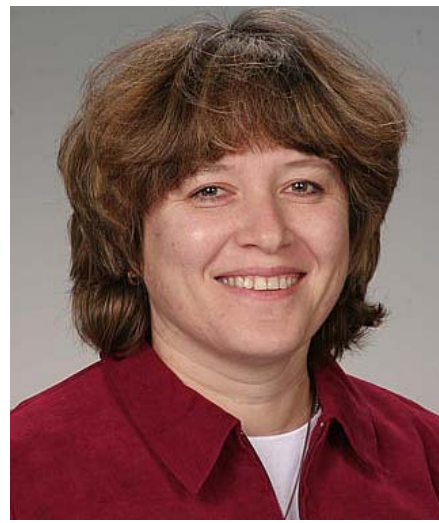

Laskin

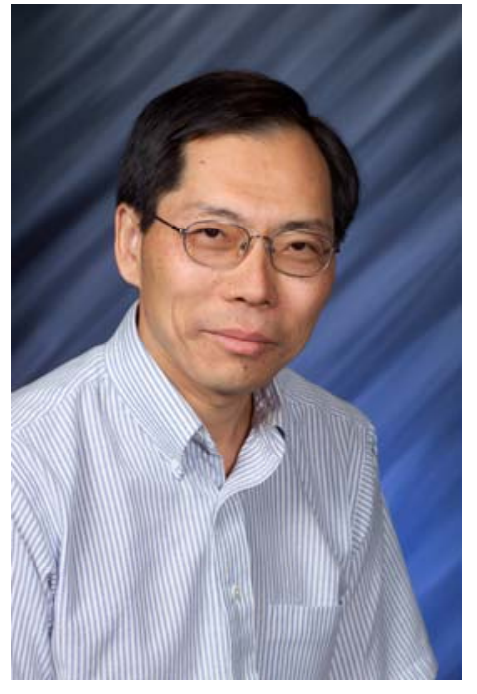

Wang
Wang accepts faculty position at Brown University. Lai-Sheng Wang (Washington State University/Pacific Northwest National Laboratory), a long-time EMSL user, has accepted a faculty position in Chemistry at Brown University. In his role at Brown University, Wang will be teaching physical chemistry and mentoring undergraduate and graduate students. Wang was selected for the faculty position based on his distinguished and innovative contributions to the field of chemistry, specifically his work on atomic clusters and gaseous multiply charged anions.

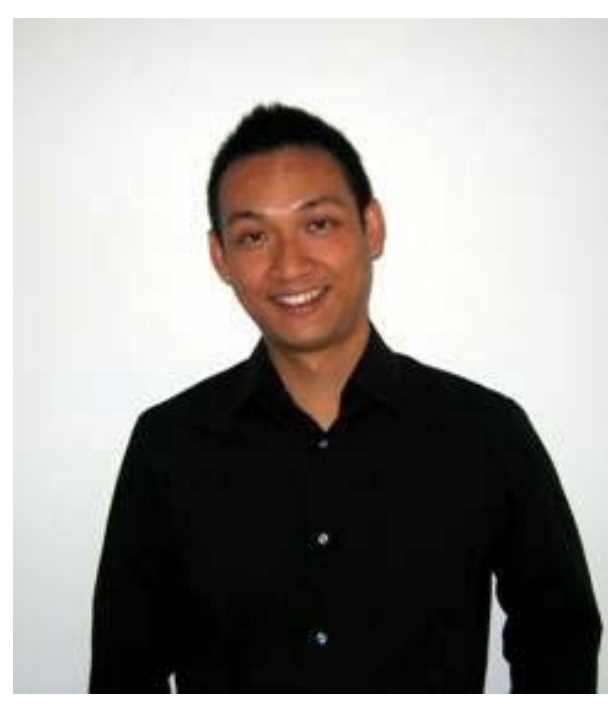

Yee
Yee garners geochemistry honors. Geochemistry research by EMSL user Nathan Yee (Rutgers University) has garnered two honors this year. First, it was selected and cited in July 2009 in Science as an Editor's Choice in the area of Geochemistry. Also, his research earned him the 2009 Houtermans' medal from the European Association for Geochemistry. Using sophisticated instruments, including electron and Mössbauer spectrometry at the Department of Energy's EMSL, Yee leads studies on how microbes reduce or mediate the toxicity of mercury and other metals. These studies are providing information to aid in designing effective contaminant sequestration technologies, key to halting toxins movement toward rivers and other waterways. The Houterman medal honors a young researcher, no more than 35 years of age, who has made exceptional contributions to geochemistry. Yee received this honor at the Goldschmidt Conference in Davos, Switzerland. 
Kukkadapu recognized as international leader in Mossbauer spectroscopy. EMSL researcher Ravi Kukkadapu recently received two international honors in the Mössbauer spectroscopy community. At the International Conference on the Applications of the Mössbauer Effect in Vienna, Austria, he was featured as an emerging leader in the Mössbauer community. He received this honor from distinguished Professor John Stevens, Director of the Mossbauer Effect Data Center, a research institute at the University of North Carolina. In addition, Kukkadapu was invited to give a keynote talk at the upcoming Clay Minerals Group of the Mineralogical Society in the United Kingdom. At this annual conference, he will discuss the effect of iron-mineral (bio)transformations on remediating contaminated aquifers that contain radioactive metals such uranium, technetium, and plutonium. Contaminated aquifers, which can occur during nuclear material production and weapons development, are a problem worldwide.

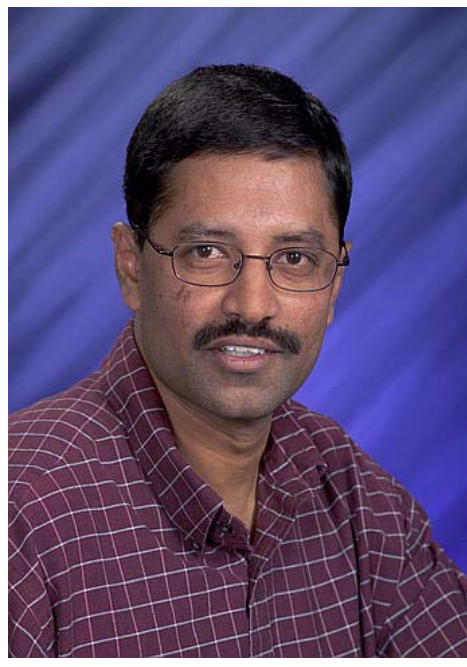

Kukkadapu

\section{Tartakovsky earns} PECASE Award. EMSL user Alexandre Tartakovsky, a computational mathematician from Pacific Northwest National Laboratory, has been recognized with a Presidential Early Career Award for Scientists and Engineers. The award honors his research on subsurface flow that addresses past and future

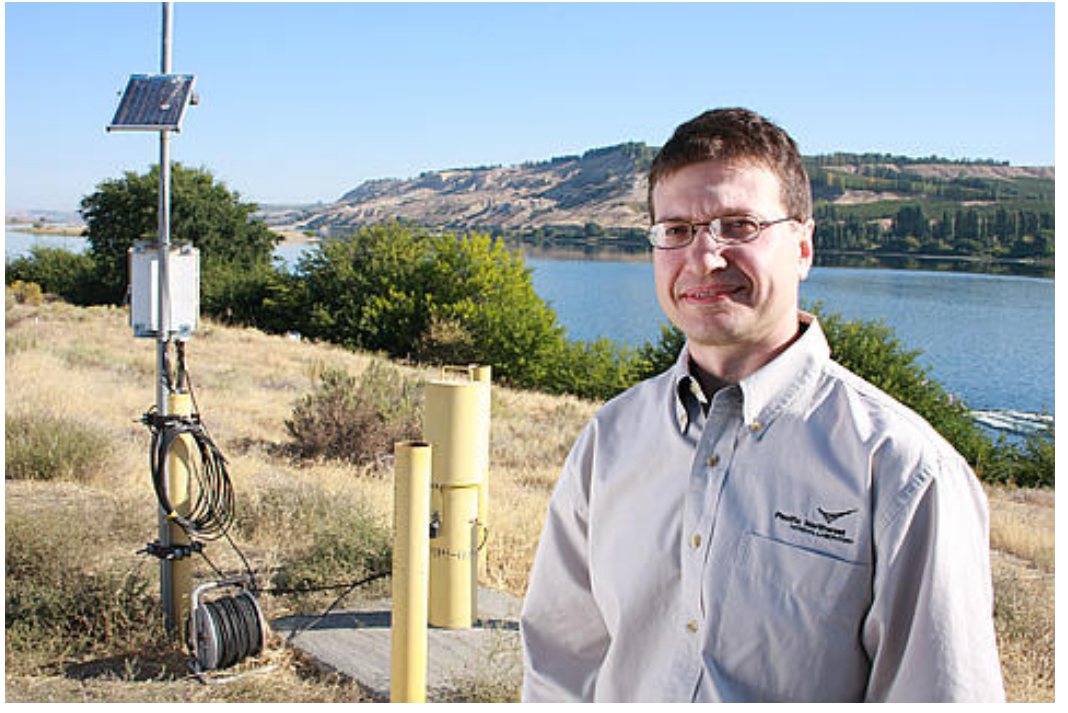

Tartakovsky energy needs: cleaning up buried nuclear or toxic contaminants and storing carbon dioxide from fossil fuels underground. Announced by the White House, the PECASE is the highest honor given by the U.S. government to scientists and engineers who are at the start of their careers. Tartakovsky earned this award for his work trying to understand how contaminants move through the subsurface, that subterranean environment made of rocks, air, liquids like water or oil, and bacteria. Ultimately, such work will help reduce the impacts that nuclear and fossil fuel energy use have on the environment. Tartakovsky develops mathematical models to help researchers clean up nuclear or toxic contaminants from past practices or help future waste managers store carbon in the subsurface. 


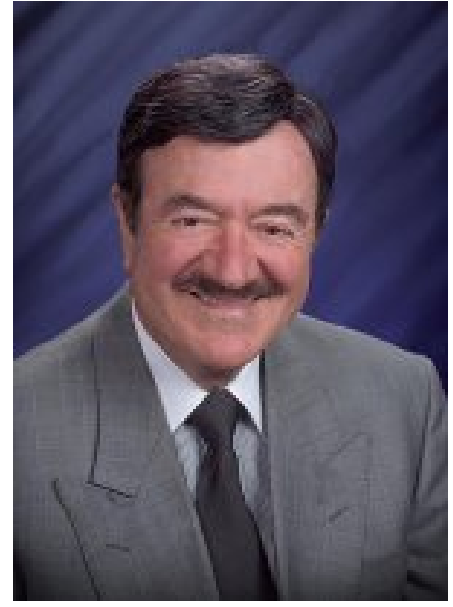

Futrell

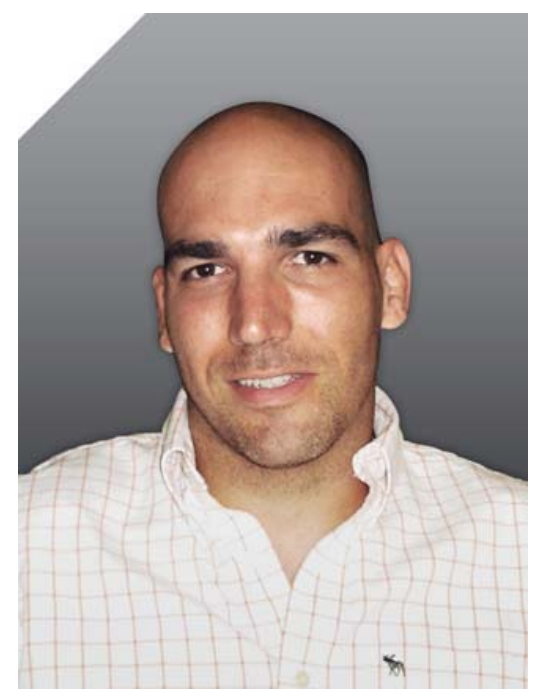

López-Ferrer

Futrell elected Fellow of ACS. Former EMSL Director Jean Futrell was selected for the inaugural class of American Chemical Society Fellows. The American Chemical Society, with more than 150,000 members, is one of the world's largest scientific organizations. This prominent honor, given to only $0.1 \%$ of the society's members, reflects Futrell's scientific achievements and his contributions to the research community. His research focuses on the theory and practice of mass spectroscopy. His work has answered fundamental questions about mass spectrometry, including the behavior of ions. He has developed or modified instruments for specialized research, creating high-pressure and chemical-ionization mass spectrometers. His invention of tandem mass spectrometry is deployed in nearly every commercial mass spectrometer.

\section{López-Ferrer receives 2008 M.T. Thomas Award. Daniel López-} Ferrer, a postdoctoral fellow in the Pacific Northwest National Laboratory's Biological Separations and Mass Spectrometry Group, was selected as the 2008 recipient of EMSL's M.T. Thomas Award for Outstanding Postdoctoral Achievement. This award acknowledges outstanding accomplishments by postdoctoral fellows who have conducted their research at EMSL during the past year. It is named in honor of Dr. M. Tom Thomas, a former PNNL staff member who played an integral role in the conceptualization of EMSL.

Dr. López-Ferrer was chosen for his remarkable contributions to high-throughput proteomics. He developed and characterized a highpressure enzymatic digestion system that can be fully integrated in an on-line process. His discovery, aided by EMSL's unmatched mass spectrometry capabilities, is opening a new realm of research possibilities for EMSL users. The methodologies he developed for processing complex biological samples have been successfully demonstrated among other EMSL projects and documented in several journal publications.

The high-pressure enzymatic digestion system developed by Dr. López-Ferrer and his colleagues in EMSL was licensed by Pressure Biosciences, Inc., a company that provides technology to control biomolecular interactions. A patent is pending, and PNNL has submitted this technology for a 2009 R\&D 100 Award. According to Battelle Fellow Dr. Richard D. Smith, who nominated Dr. López-Ferrer for the award, Daniel "has been the creative force behind a number of technical advances that benefit EMSL's high-throughput proteomics capability by overcoming the bottleneck in throughput at the front end of the workflow." Dr. López-Ferrer received his Ph.D in Biochemistry from the Autonomous University of Madrid, Madrid, Spain, in 2006. He has been a PNNL postdoctoral research associate since 2006. 


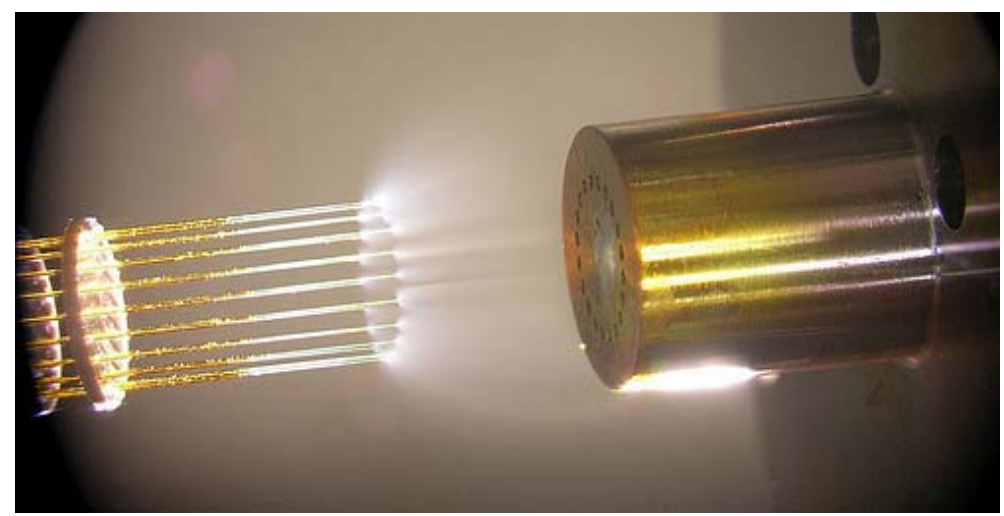

Figure 1. The Ultrasensitive ESI-MS Source and Interface developed at EMSL provides at least a 40-fold increase in mass spectrometry sensitivity relative to commercially available instruments. This ultrasensitive instrument can aid in biological and environmental studies, among others.
Integrating resources at EMSL helps scientists win R\&D 100

Award. When characterizing small, hard-to-acquire samples, scientists need sensitive instruments that can provide highly accurate data. By integrating a combination of inventions and approaches at EMSL, scientists achieved at least a 40 -fold increase in the sensitivity of mass spectrometers and earned an R\&D 100 Award for their efforts. Known as the Oscars of the research world, $\mathrm{R} \& \mathrm{D}$ magazine presents the awards annually to the 100 most innovative scientific and technical breakthroughs

of the year. The Ultrasensitive Electrospray Ionization-Mass Spectrometry (ESI-MS) Source \& Interface was developed by EMSL users Dick Smith, Ryan Kelly, Jason Page, and Keqi Tang of Pacific Northwest National Laboratory. The ESI-MS can measure amounts of compounds in a sample very precisely, even when very little material is available. This sensitivity is especially important when sample sizes are limited, such as from microbiopsies of human tissue or proteins extracted from small sediment samples. Scientists at PNNL are using the improved sensitivity in fundamental biological and environmental studies, to develop biomarkers for early disease diagnosis, and to discover drug targets. Further, they are collaborating with a major vendor of mass spectrometry instruments to further explore the benefits of the new ESI-MS system. 


\section{Journal Covers}

During Fiscal Year 2009, research by EMSL users and staff was highlighted on 14 scientific journal covers. The following describe the research that led to these covers.
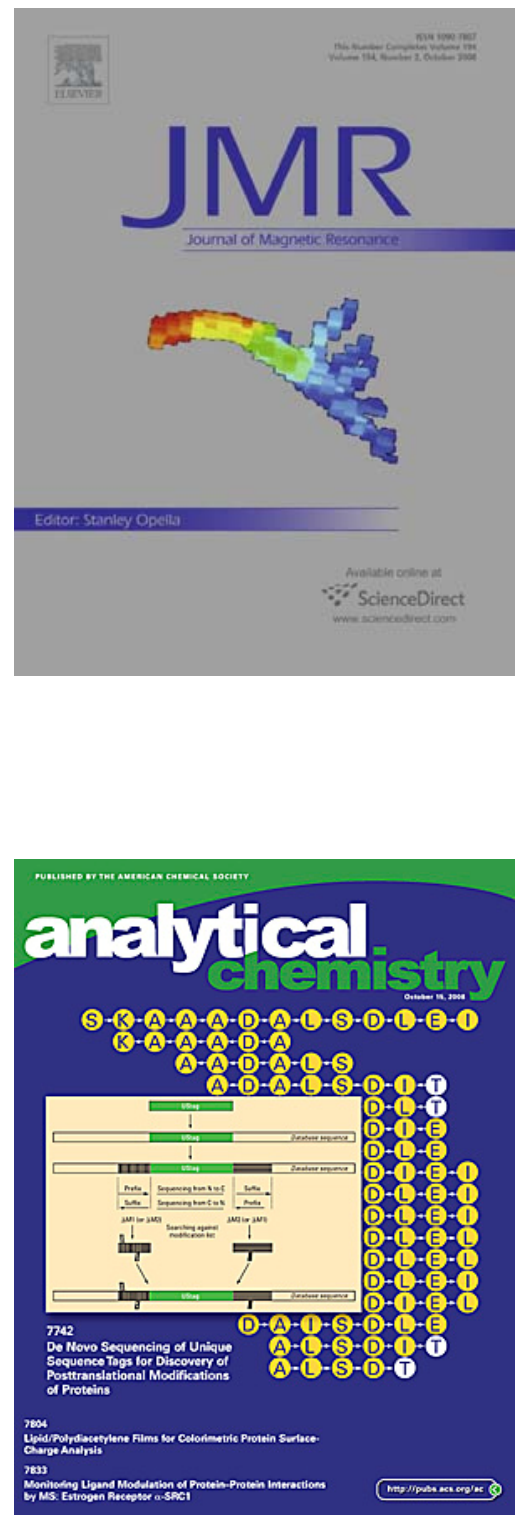

Airflow patterns in the lung not only determine how well you breathe but also how inhaled materials such as airborne pollutants or aerosolized drugs get distributed inside your body. EMSL users from Pacific Northwest National Laboratory and the University of Utah have pioneered a new Magnetic Resonance Imaging method for visualizing inhaled airflow patterns. The method uses hyperpolarized ${ }^{3} \mathrm{He}$ (helium) gas as an inert tracer for visualizing inhaled air speed and direction at each location within the complex, three-dimensional airways of live laboratory rat lungs.

Funding Source: National Institutes of Health

Journal of Magnetic Resonance, 194(1):182-191. October 2008. "MR Imaging of Apparent ${ }^{3} \mathrm{He}$ Gas Transport in Narrow Pipes and Rodent Airways."

Authors: Kevin R. Minard, Richard E. Jacob, Daniel R. Einstein, Andrew P. Kuprat, Richard A. Corley, Pacific Northwest National Laboratory

Gernot Laicher, University of Utah

EMSL researchers and users have developed a novel strategy that expands protein identifications beyond those predicted from genomic sequences. Their approach can be used to probe posttranslational —or chemical — modifications of proteins, adding knowledge to studies of heart disease, cancer, neurodegenerative diseases, diabetes and essentially any other area of biology.

Funding Source: EMSL Intramural Research Program, DOE Office of Biological and Environmental Research, National Institutes of Health National Center for Research Resources

Analytical Chemistry, 80(20):7742-7754. October 15, 2008. "De novo Sequencing of Unique Sequence Tags for Discovery of PostTranslational Modifications of Proteins."

Authors: Yufeng Shen, Gordon A. Anderson, Richard D. Smith, Pacific Northwest National Laboratory

Nikola Tolić, Kim K. Hixson, Samuel O. Purvine, EMSL 


\section{ChemComm}
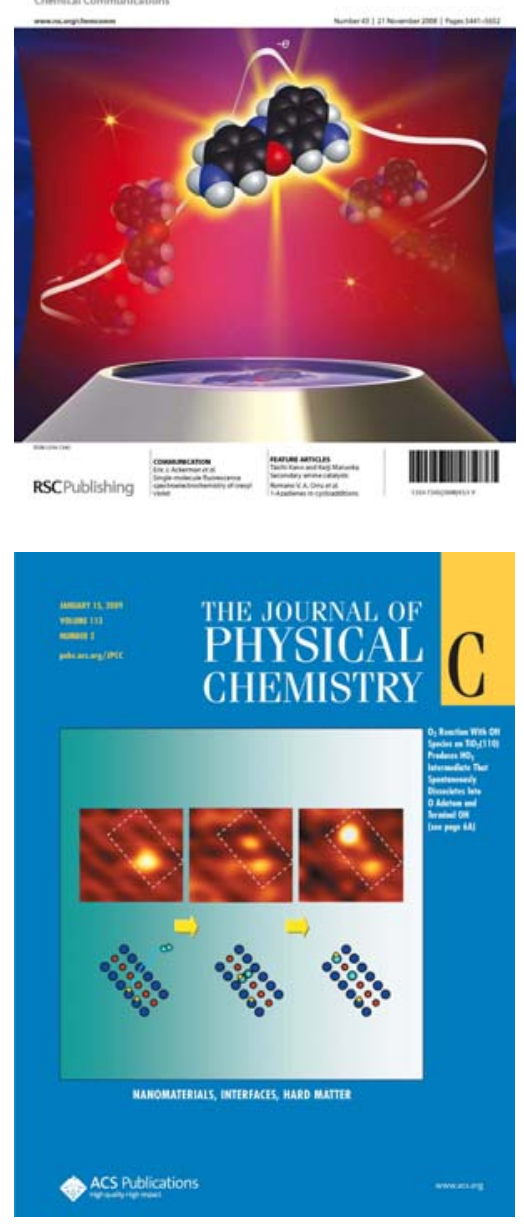

EMSL users from Pacific Northwest National Laboratory report a new path to study electron transfer dynamics by coupling singlemolecule microscopy with an electrochemical instrument-a potentiostat. This enables them to better understand electron transfer reactions, which in turn could lead to understanding reactions common to biofuel production and solar energy conversion.

Funding Source: DOE Office of Basic Energy Sciences

Chemical Communications, 43:5490-5492. November 21, 2008.

"Single-Molecule Fluorescence Spectroelectrochemistry of Cresyl Violet."

Authors: Chenghong Lei, Dehong Hu and Eric J. Ackerman, Pacific Northwest National Laboratory

Expected but elusive - that's how scientists described two intermediates that may play an important role in the reaction that turns water into hydrogen. Now, thanks to the work of EMSL researchers and users from Pacific Northwest National Laboratory, these intermediates are no longer a mystery. In a recent article featured on the cover of the Journal of Physical Chemistry C, the researchers provided images of two intermediates that occur during the reaction that changes water into hydrogen.

Funding Source: DOE Office of Basic Energy Sciences

Journal of Physical Chemistry C, 113(2):666-671. January 15, 2009. "Imaging Consecutive Steps of $\mathrm{O}_{2}$ Reaction with Hydroxylated $\mathrm{TiO}_{2}(110)$ : Identification of $\mathrm{HO}_{2}$ and Terminal $\mathrm{OH}$ Intermediates."

Authors: Yingge Du, Igor Lyubinetsky, EMSL N. Aaron Deskins, Zhenrong Zhang, Zdenek Dohnálek, Michel Dupuis, Pacific Northwest National Laboratory 

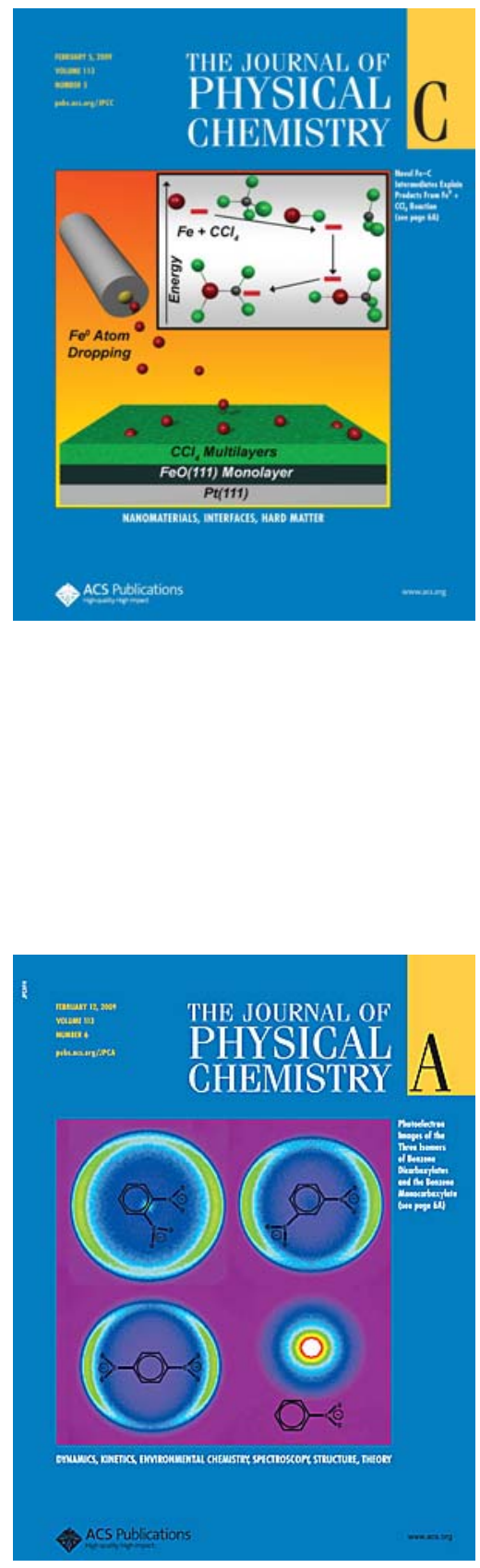

Novel $\mathrm{Fe}-\mathrm{C}$ intermediates explain products from $\mathrm{Fe}^{0}+\mathrm{CCl}_{4}$ reaction. The environmentally important reaction between $\mathrm{Fe}^{0}$ and $\mathrm{CCl}_{4}$ has been studied experimentally using an "atom dropping" technique and theoretically via density functional theory calculations. Temperature programmed desorption and X-ray photoelectron spectroscopy results show that the reactivity is strongly size dependent with isolated $\mathrm{Fe}$ atoms and small clusters significantly more reactive than larger particles. The product branching ratio depends strongly on the $\mathrm{Fe}$ coverage with $\mathrm{FeCl}_{3}$ and $\mathrm{OCCl}_{2}$ dominant for isolated $\mathrm{Fe}$ atoms and $\mathrm{FeCl}_{2}$ and $\mathrm{CO}$ dominant for Fe nanoparticles. The experimentally observed trends are consistent with the theoretical calculations that predict novel intermediate species featuring $\mathrm{Fe}-\mathrm{C}$ bonds. The calculations support a reaction mechanism for the formation of $\mathrm{FeCl}_{3}$ and $\mathrm{CO}$, products that cannot be explained by a simple $\mathrm{Cl}$ abstraction model.

Funding Source: DOE Office of Basic Energy Sciences

Journal of Physical Chemistry C, 113(5):1818-1829. February 5, 2009. "Reactivity of $\mathrm{Fe} 0$ Atoms, Clusters, and Nanoparticles with $\mathrm{CCl}_{4}$ Multilayers on $\mathrm{FeO}(111)$."

Authors: Gareth S. Parkinson, Zdenek Dohnalek, R. Scott Smith, Bruce D. Kay, Pacific Northwest National Laboratory

Photoelectrons emitted from multiply charged anions (MCAs) carry information of the intramolecular Coulomb repulsion (ICR), which is dependent on molecular structures. Using photoelectron imaging, we observed the effects of ICR on photoelectron angular distributions (PAD) of the three isomers of benzene dicarboxylate dianions $\mathrm{C} 6 \mathrm{H} 4(\mathrm{CO} 2) 2$ 2- (o-, m- and p-BDC2-). Photoelectrons were observed to peak along the laser polarization due to the ICR, but the anisotropy was the largest for p-BDC2-, followed by the $\mathrm{m}$ and o-isomer. The observed anisotropy is related to the direction of the ICR or the detailed molecular structures, suggesting that photoelectron imaging may allow structural information to be obtained for complex multiply charged anions.

Funding Source: DOE Office of Basic Energy Sciences, National Science Foundation.

Journal of Physical Chemistry A, 113(6):945-948. February 12, 2009. "Photoelectron Angular Distribution and Molecular Structure in Multiply Charged Anions."

Authors: Xiao-Peng Xing, Xue-Bin Wang and Lai-Sheng Wang, Washington State University/Pacific Northwest National Laboratory 

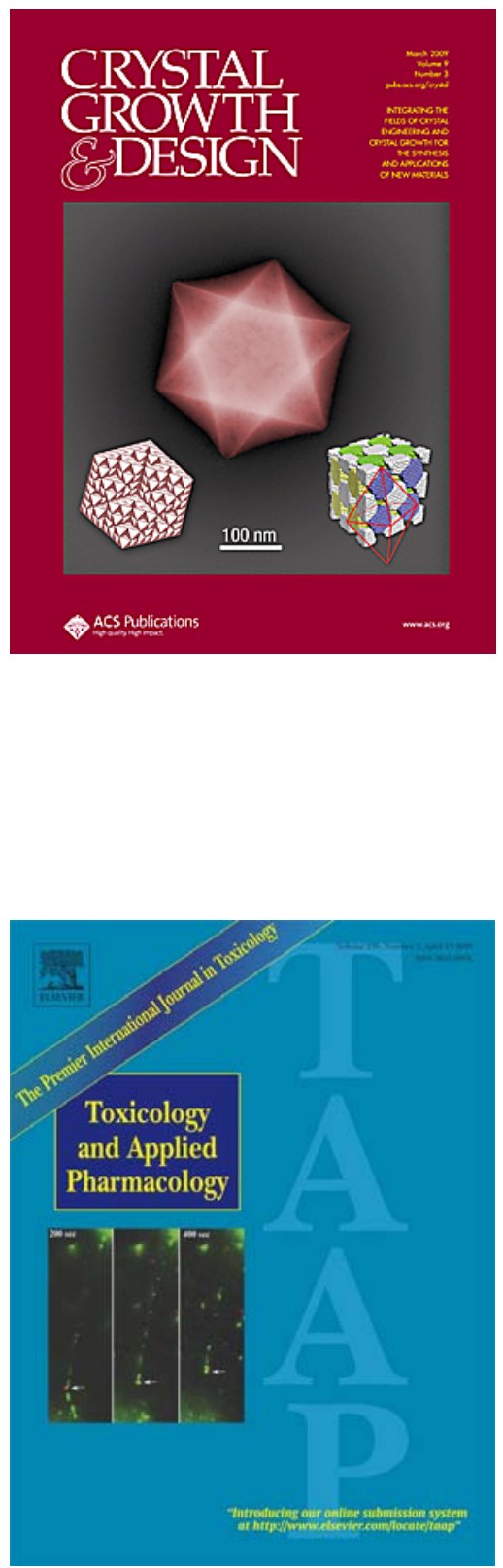

The evolution of a self-assembled, oxide superoctahedral structure has been elucidated for the first time, and this unique finding was featured on the cover of Crystal Growth and Design - a journal ranked first in the field based on impact factor. For these studies, researchers at the Department of Energy's EMSL and their collaborators from the University of Central Florida and the Defence Academy of the United Kingdom used a combination of experimental tools at UCF and EMSL as well as theoretical tools at the Cambridge-Cranfield HPC facility to study cerium oxide. Because cerium oxide is crucial for solid oxide fuel cells, solar cells, catalysis, and biomedicine, understanding its nanostructures is vital to improve their performance in those applications.

Funding Source: National Science Foundation

Crystal and Growth and Design, 9(3):1614-1620. March 2009. "Symmetry-Driven Spontaneous Self-Assembly of Nanoscale Ceria Building Blocks to Fractal Superoctahedra."

Authors: Satya Kuchibatla, EMSL, University of Central Florida AS Karakoti, H Heinrich, S Seal, University of Central Florida DC Sayle, Defence Academy of the United Kingdom

By combining single-molecule microscopy and nanoparticle engineering, EMSL users from Pacific Northwest National Laboratory showed how nano-sized particles interact with lung cells. Their results, which were published in a recent cover article in Toxicology and Applied Pharmacology, provide insights about the mechanisms underlying of inhaled nanomaterials.

Funding Source: EPA STAR grant, the Air Force Research Laboratory grant FA8650-05-1-5041 to ONAMI Safer Nanomaterials and Nanomanufacturing Initiative, and the Environmental Biomarkers Initiative at the Pacific Northwest National Laboratory.

Toxicology and Applied Pharmacology, 236(2):210-220. April 15, 2009. "Syndecan-1 Mediates the Coupling of Positively Charged Submicrometer Amorphous Silica Particles with Actin Filaments across the Alveolar Epithelial Cell Membrane."

Authors: Galya Orr, David J. Panther, Kaylyn J. Cassens, Jaclyn L. Phillip, Barbara J. Tarasevich, Joel G. Pounds, Pacific Northwest National Laboratory 

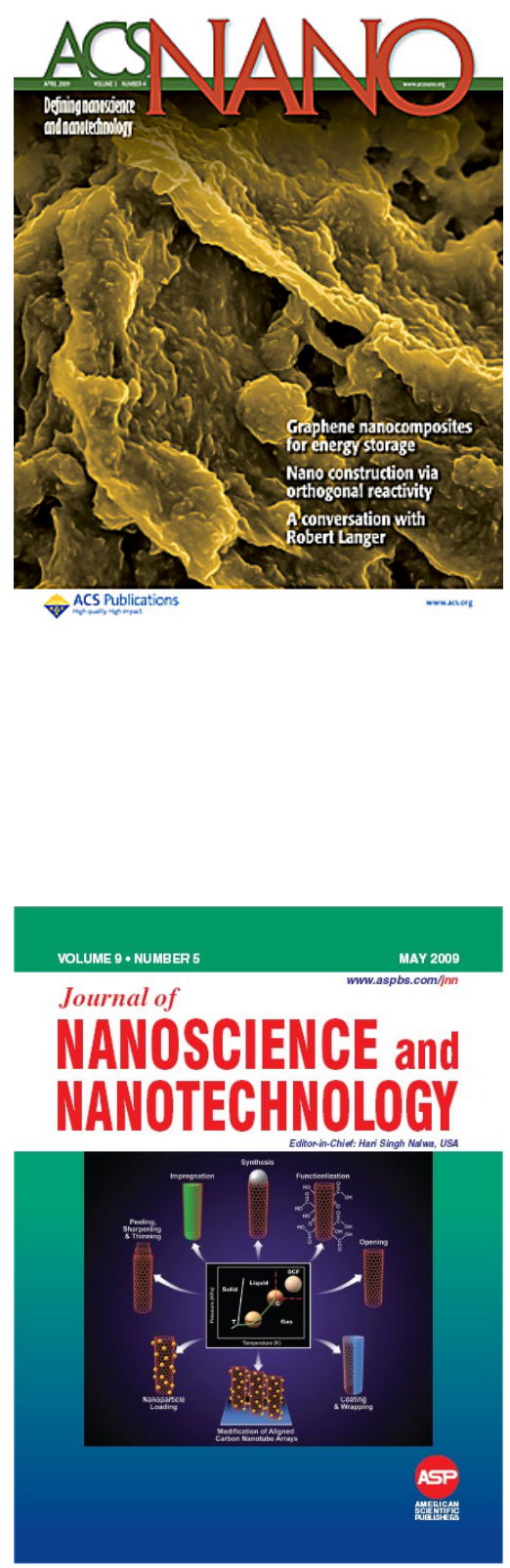

High capacity, safe batteries are needed for efficient hybrid or electrical vehicles and for storing and releasing electricity from intermittent power sources like wind turbines and solar panels. That's where an innovation by scientists at Pacific Northwest National Laboratory, EMSL, and Princeton University comes in. The researchers devised a method for building tiny titanium oxide and carbon structures that greatly improve the performance of lithium ion batteries. This new material stores twice as much electricity at high charge/discharge rates as batteries that don't use it.

Funding Source: PNNL Laboratory-Directed Research and Development funds, DOE Office of Basic Energy Sciences

ACS Nano, 3(4):907-914. April 28, 2009. "Self-Assembled $\mathrm{TiO}_{2}-$ Graphene Hybrid Nanostructures for Enhanced Li-Ion Insertion."

Authors: Donghai Wang, Daiwon Choi, Juan Li, Zhenguo Yang, Zimin Nie, Rong Kou, Dehong Hu, Jiguang Zhang, Jun Liu, Pacific Northwest National Laboratory

Chongmin Wang, Laxmikant V. Saraf, EMSL

Ilhan A. Aksay, Princeton University

Building carbon tubes so small that they make red blood cells look like blimps provides a unique set of challenges, but these difficulties may be outweighed by the innovative properties these structures offer. Researchers at Jinhua College of Profession and Technology, Zhejiang Normal University, and Pacific Northwest National Laboratory reviewed studies of supercritical fluids, which exhibit the properties of gas and a liquid at the same time, and carbon nanotubes. This invited review was published in the May 2009 issue of the Journal of Nanoscience and Nanotechnology. Artwork from the review graced the cover.

Funding Source: PNNL Laboratory Directed Research and Development Program. In addition, Dr. Ye received funds from a PNNL fellowship program to work at PNNL.

Journal of Nanoscience and Nanotechnology, 9(5):2781-2794. May 2009. "Supercritical Fluid Assisted Synthesis and Processing of Carbon Nanotubes."

Authors: Sufang Ye, Jinhua College of Profession and Technology Fengmin $\mathrm{Wu}$, Zhejiang Normal University Yuehe Lin, Pacific Northwest National Laboratory 

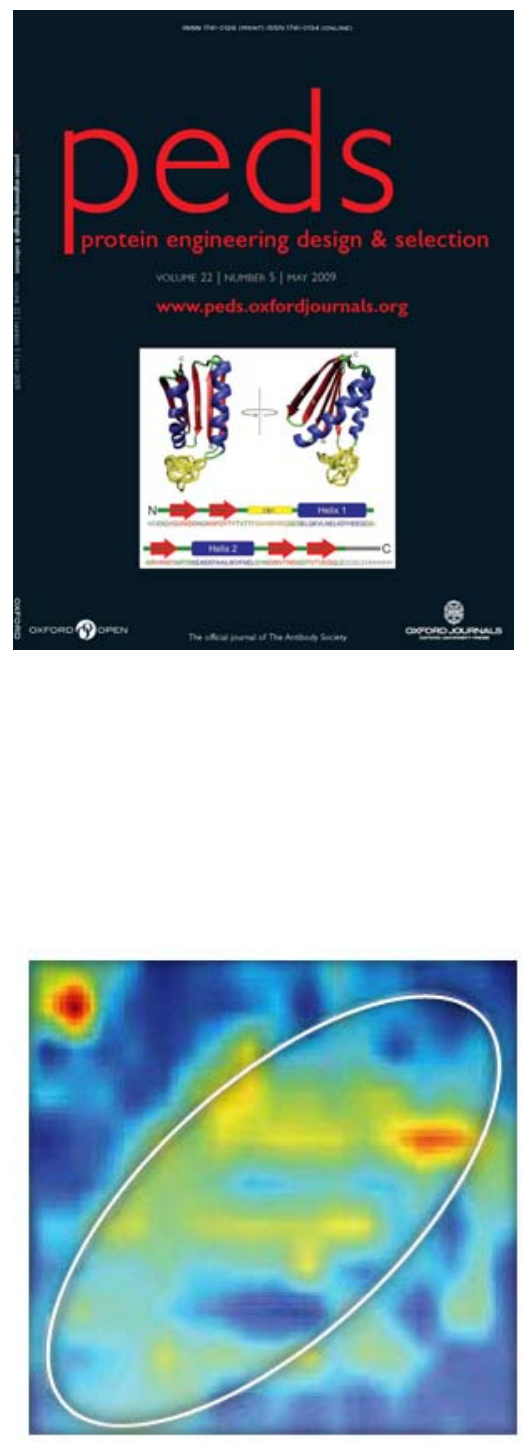

Move over, Tommy Hilfiger. Top $7_{\mathrm{CB} 1}$, designed by EMSL users from Pacific Northwest National Laboratory, is grabbing the spotlight. This synthetic protein can specifically bind a protein targeted by the human immunodeficiency virus, or HIV, that can lead to AIDS. Top $7_{\mathrm{CB} 1}$ can also be used as an inexpensive and effective alternative to antibodies.

Funding Source: U.S. Department of Energy, Laboratory Directed Research and Development funding through the Biomolecular Systems Initiative

Protein Engineering Design and Selection, 22(4):325-332. May 2009. "Engineering an Ultra-Stable Affinity Reagent Based on Top7."

Authors: Curt B. Boschek, Thereza A. Soares, TP Straatsma, Heather E. Engelmann, Cheryl L. Baird, Pacific Northwest National Laboratory

David O. Apiyo, Pacific Northwest National Laboratory, Immunoassay Business Center

Noah B. Pefaur, Pacific Northwest National Laboratory, ZymoGenetics

An international team used surface science and imaging capabilities at the Department of Energy's EMSL to determine the location, with nanoscale resolution, of MtrC and $\mathrm{OmcA}$ - two Shewanella oneidensis MR-1 surface proteins that can affect environmental quality. Carried out, in part, as a contribution to EMSL's Biogeochemistry Grand Challenge, the team's imaging studies offer a deeper understanding of the role MtrC and OmcA play in electron exchange and may lead to enhanced bioremediation methods.

Funding Source: DOE Office of Basic Energy Sciences Geosciences Research Program, DOE Office of Biological and Environmental Research Genomics-Genomes to Life Program, National Science Foundation. Performed in part under EMSL's Biogeochemistry Grand Challenge.

Applied and Environmental Microbiology, 75(9):2931-2935. May 1, 2009. "Antibody-Recognition Force Microscopy Shows that Outer Membrane Cytochromes OmcA and MtrC are Expressed on the Exterior Surface of Shewanella oneidensis MR-1."

Authors: Brian H. Lower, Steven K. Lower, The Ohio State University

Ruchirej Yongsunthon, Corning, Inc.

Liang Shi, Catherine L. Reardon, Grigoriy E. Pinchuk, Timothy C. Droubay, Pacific Northwest National Laboratory

Linda Wildling, Hermann J. Gruber, Johannes Kepler University of Linz

Nicholas S. Wigginton, Ecole Polytechnique Federale de Lausanne Jean-Francois Boiley, Umea University 

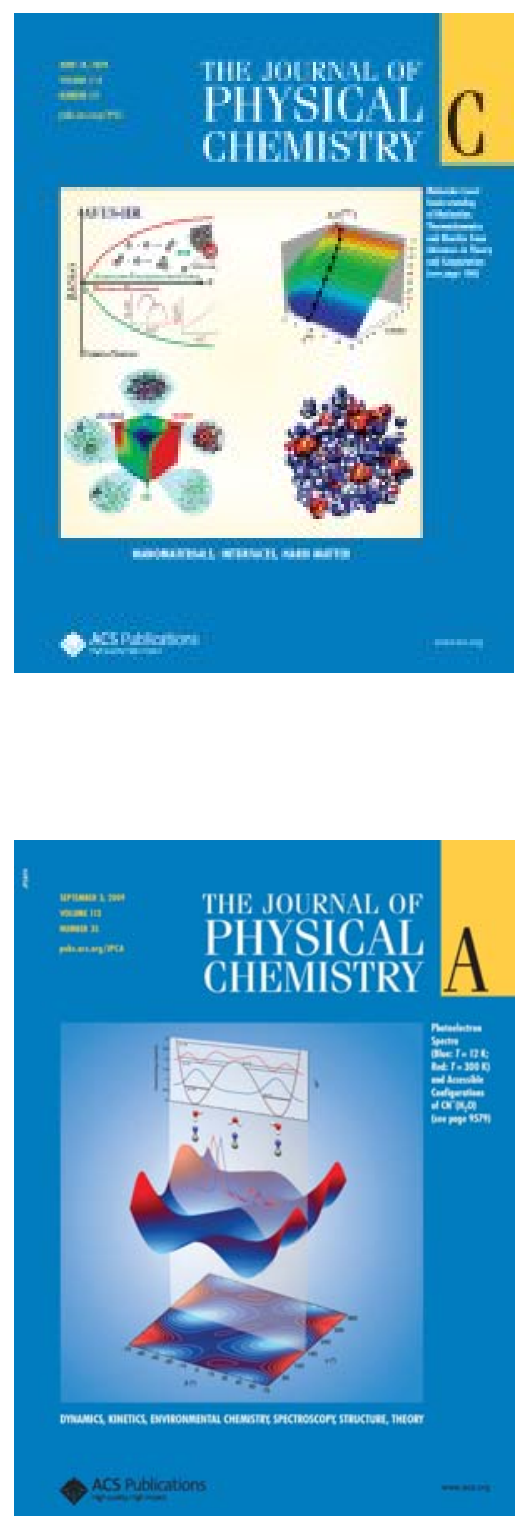

Solving the nation's energy crisis requires designer materials, such as crystals and catalysts, with very specific properties. Creating these properties requires scientists to design new materials molecule by molecule. To make designing these materials a reality, scientists at Pacific Northwest National Laboratory, Louisiana State University, and the University of Minnesota developed methods to determine how fast clusters of molecules form and their corresponding stability.

\section{Funding Source: DOE's Office of Basic Energy Sciences}

Journal of Physical Chemistry C, 113(24):10354-10370. June 18, 2009. "Thermodynamics and Kinetics of Nanoclusters Controlling Gas-to-Particle Nucleation."

Authors: Shawn Kathmann, Greg Schenter, Bruce C. Garrett, Pacific Northwest National Laboratory

Bin Chen, Louisiana State University

J Ilja Siepmann, University of Minnesota

Scientists have long known that molecules dance about as the temperature rises, but now researchers know the exact steps that water takes with a certain molecule. Results with small, electrically charged cyanide ions and water molecules reveal that water zips around ions to a greater extent than expected. The findings improve our understanding of a chemical interaction important in atmospheric sciences.

Funding Source: DOE's Office of Basic Energy Sciences

Journal of Physical Chemistry C, 113:9579-9584. September 3, 2009. "Observation of a Remarkable Temperature Effect in the Hydrogen Bonding Structure and Dynamics of the $\mathrm{CN}^{-}\left(\mathrm{H}_{2} \mathrm{O}\right)$ Cluster. "

Authors: Xue-Bin Wang, Lai-Sheng Wang, Washington State University, Pacific Northwest National Laboratory Jasper C. Werhahn, Alfred Laubereau, Technische UniVersita" $t$ Munchen

Karol Kowalski, EMSL

Sotiris S. Xantheas, Pacific Northwest National Laboratory 


\section{Outreach Activities}

The following outreach activities were conducted by EMSL staff in Fiscal Year 2009:

Three users named to Visiting Scientist Program. Three longtime EMSL users have been appointed as participants of the Wiley Visiting Scientist Program, which is designed to recognize, reward, and encourage distinguished scientists to come to EMSL for extended periods of time and make significant contributions to the EMSL user program by providing input to and recommendations on the path forward for EMSL.

- Walter Ermler is professor of chemistry at the University of Texas at San Antonio. At EMSL, he will work to integrate his CRENB spin-orbit operators with EMSL's NWChem software suite basis set and the EMSL Basis Set Exchange Library for the entire periodic system. The availability of these spin-orbit potentials in NWChem will directly support geochemistry and subsurface science research by enabling calculations that can provide more accurate results for those studying the structures and reaction mechanisms of heavy-element contaminants at various interfaces at the molecular scale. He will also develop collaborations in the area of modeling the electronic structure and spectroscopic properties of trans-uranium molecules and clusters using highly accurate relativistic methodologies.

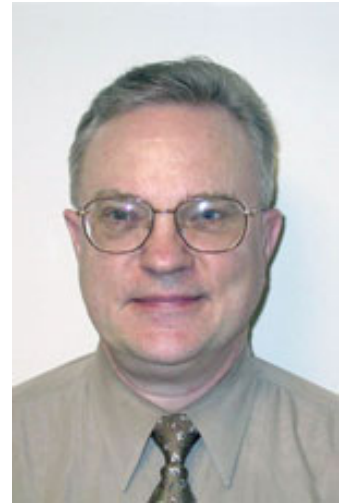

Ermler

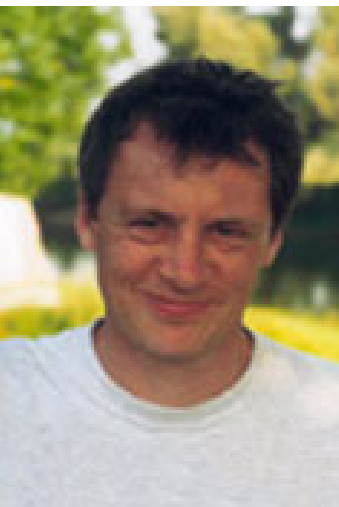

Farnan

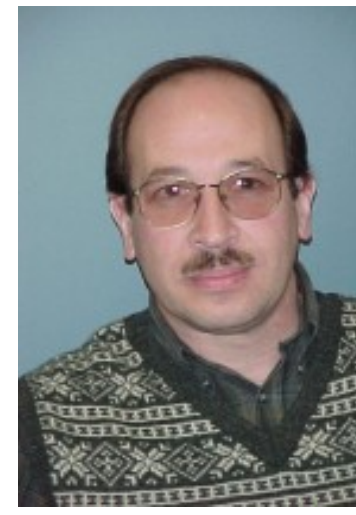

Shluger

Applications for the Wiley Visiting Scientist Program are accepted quarterly, and appointments include a stipend for travel and per diem. For more information, see http://www.emsl.pnl.gov/news/awards/visiting_scientist.jsp. 
Chambers and Cho named Wiley Research Fellows. EMSL users Scott Chambers and Herman Cho (Pacific Northwest National Laboratory) are the first Wiley Research Fellows. The program recognizes scientists who make significant contributions to EMSL outside of their individual research efforts. As Fellows, Chambers and Cho will contribute to EMSL decision-making processes, including serving on advisory committees and participating on new capability proposals. In recognition of their work, they can request time on high-demand instruments normally reserved for EMSL staff.

Chambers was selected for his scientific and technological leadership in growing well-defined oxide films and surfaces. Such materials are of interest in photocatalysis, surface geochemistry, and other scientific areas. As a thin films expert, Chambers spends time assisting EMSL users with designing experiments and preparing samples. As a Wiley Research Fellow, he will lead the design and construction of EMSL's third generation oxygen plasma assisted molecular beam epitaxy system. The system will enable growing oxide films of increasing complexity. Chambers is a Laboratory Fellow at Pacific Northwest National Laboratory.

Cho was chosen for his leadership in developing EMSL's radiological nuclear magnetic resonance (NMR) capabilities. These capabilities are critical in studies such as radioactive waste storage forms and radionuclide migration in the subsurface. As a Wiley Research Fellow, he will provide advice and support to the design and construction of EMSL's radiological annex. The annex will provide specialized instruments, including NMR spectroscopy, for the characterization and analysis of radiological

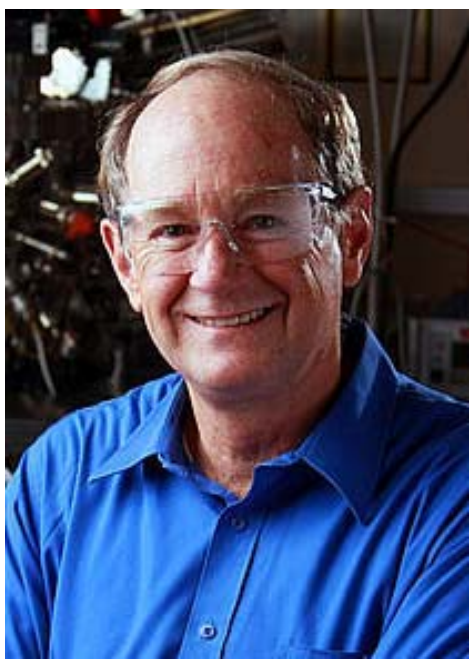

Chambers

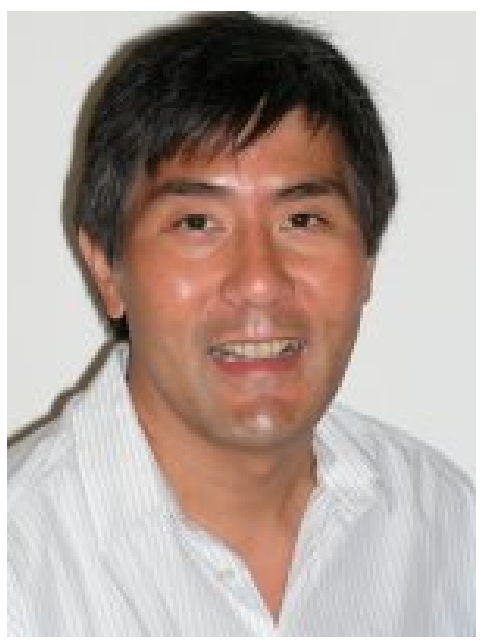

Cho materials. Cho is a senior research scientist at PNNL. The Fellows program is named after William R. Wiley. A Laboratory Director at PNNL, he helped take EMSL from an idea to a world-class facility. Wiley felt that EMSL would help the world respond to scientific challenges that depended on fundamental science. 
EMSL co-hosts Migration '09, the $12^{\text {th }}$ International Conference on the Chemistry and Migration Behaviour of Actinides and Fission Products in the Geosphere, with PNNL and Washington State University. Nearly 300 researchers from around the world attended the conference, held September $20-25,2009$. They attended sessions on topics such as Geochemical and Transport Modeling, Solid Solution and Secondary Phase Formation, SolidWater Interface Reactions, and International Research Programs, as well as a special Hanford Site

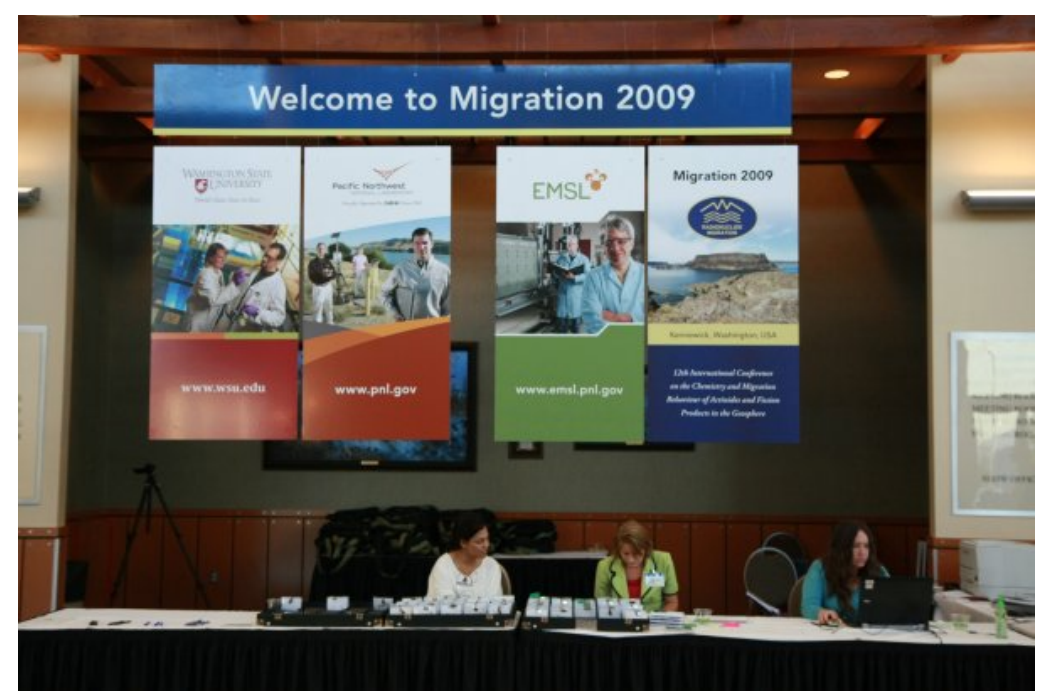

EMSL co-hosted Migration '09 on September 20-25, 2009. Session. The participants also toured EMSL as part of the conference. The conference has previously been held in venues such as Munich, Korea, France, and Japan.

National meeting presence. EMSL had a "booth" presence at the GTL:Genomes to Life Contractor's Meeting in February 2009. Outreach targeted BER principal investigators and the research/capabilities available at EMSL. EMSL also sponsored refreshments at the $51^{\text {st }}$ Annual Rocky Mountain Conference on Analytical Chemistry in July 2009 and had a presentation and posters at the ERSP PI Meeting in April 2009.

\section{EMSL launches a} Facebook page designed to target the $18-35$ yearold demographic. The page is designed to provide updates about science conducted at EMSL as well as provide a forum for discussion. EMSL currently has nearly 500 fans and growing.

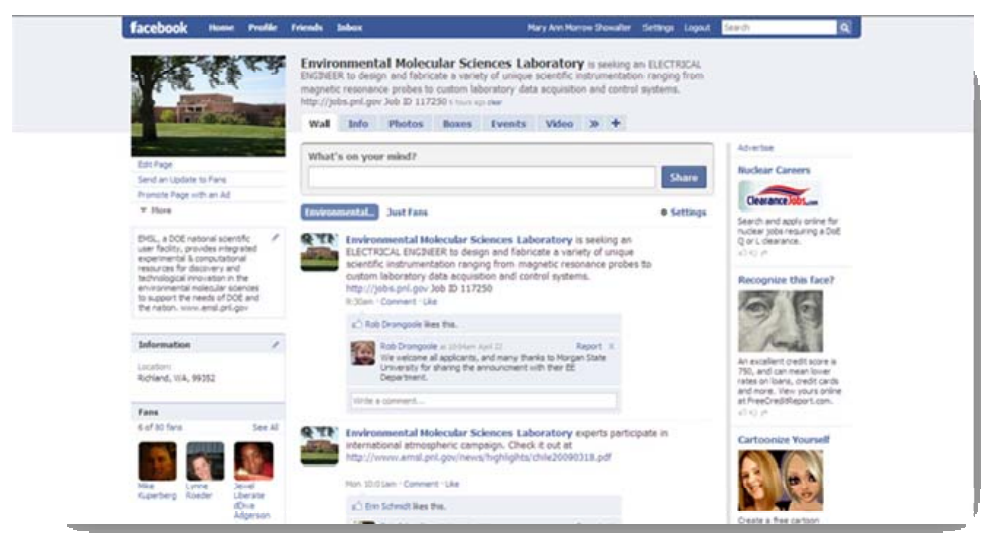

EMSL launched its Facebook page in April 2009. 
EMSL launches a series of videos, including a video outlining the capabilities and science enabled by the Focused Ion Beam-Scanning Electron Microscope, and four "Interview with an EMSL User" videos where users discuss their research and how EMSL enables their research. The videos were leveraged on EMSL's website, EMSL's Youtube channel and Facebook page, as well as the 5,000+ member list serve.

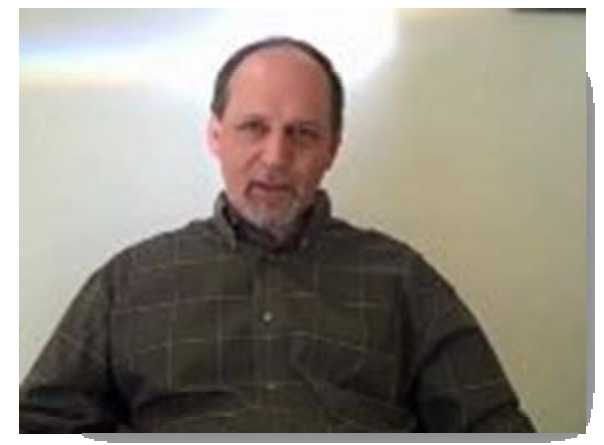

EMSL user Geoff Marcy discusses his research on one of EMSL's Interview with a User videos.

\section{A total of 160 tours were given in EMSL, ranging from local students to high-level political and DOE officials. Stops on the tour include the supercomputer, the NMR lab, laser and surface science capabilities, and the mass spectrometry lab. This includes Take Your Sons and Daughters to Work Day on April 30, 2009.}

\section{User Surveys}

In Fiscal Year 2009, EMSL conducted two user surveys to determine satisfaction with availability and performance of facilities and equipment, assistance of technical and

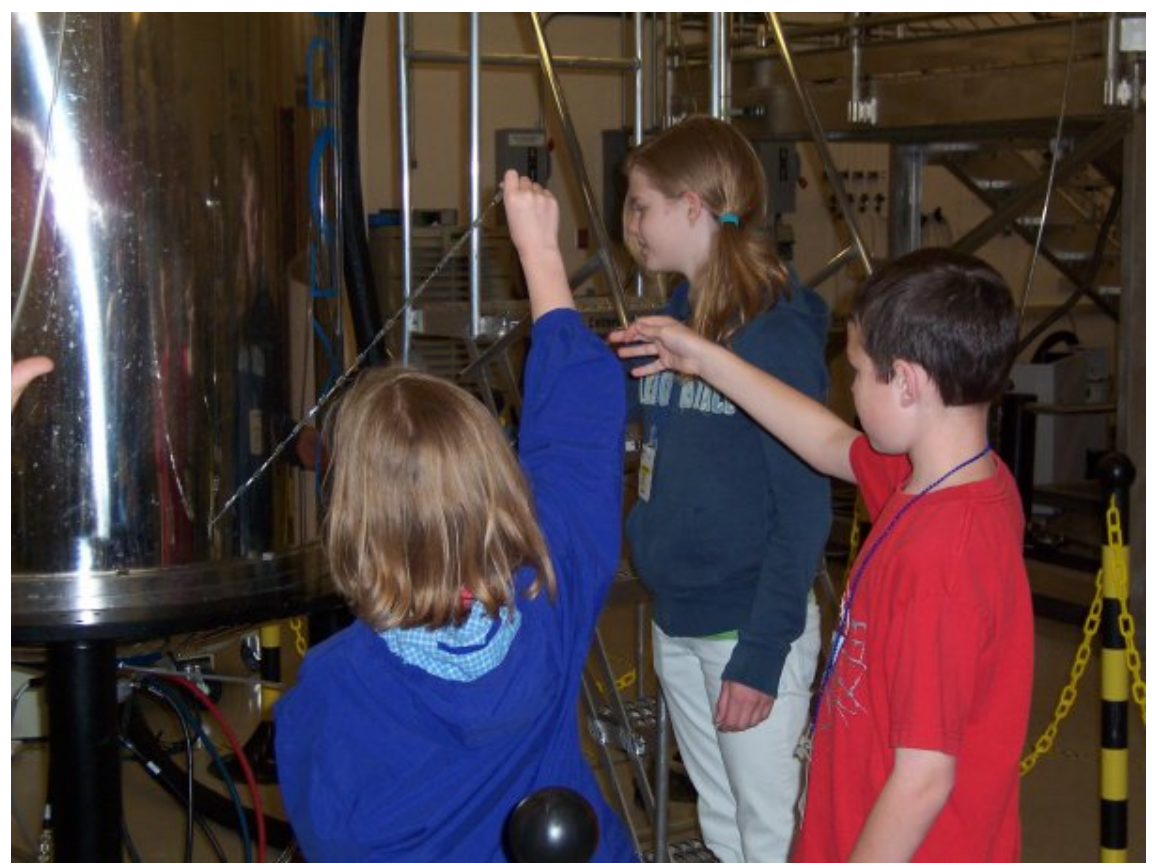

EMSL NMR capabilities and their magnetic properties "wow" some of the kids who attended Take Your Sons and Daughters to Work Day on April 30.

administrative staff, training and safety procedures, and the user proposal process.

- In October 2009 (results not yet published), 211 responses (35.9\%) were received out of 588 surveys sent out. The responses constituted a $92.4 \%$ satisfaction rate.

- In April 2009 (http://www.emsl.pnl.gov/access/surveys/), 248 responses (47.6\%) were received out of 521 surveys sent out. The responses constituted a $93.1 \%$ satisfaction rate.

Specific comments from users and responses by EMSL to those comments are available at the web addresses listed above. 


\section{Biological Interactions and Dynamics}

Understanding and optimizing the response of biological systems to their environment can have a significant impact on achieving viable solutions to several problems of national concern. For example, a deep knowledge of anaerobic microbial metabolism can improve national efforts in environmental cleanup and site stewardship, and help provide clean and secure energy. Molecular-level measurements and insights into biochemical processes are also foundational to building predictive computational models that would improve our ability to use microbes effectively and safely as an approach to mitigate the impacts of energy-production activities on the environment and human health and to extend basic scientific research.

Recent advances in whole-genome sequencing of a variety of organisms and improvements in highthroughput instrumentation have contributed to a rapid transition of the biological research paradigm towards understanding biology at a systems level. As a result, biology is evolving from a descriptive to a quantitative, ultimately predictive science where the ability to collect and productively use large amounts of biological data is crucial. Understanding how the ensemble of proteins in cells gives rise to biological outcomes is fundamental to systems biology. Advances in systems-level research will require new technologies and approaches to measure and track the temporal and spatial disposition of proteins in cells and how protein complexes give rise to specific activities.

To help facilitate the transition of biology to a more quantitative science, EMSL encourages user proposals with a focus on the following key topical areas:

- Understanding the protein and metabolite composition of cells as well as the activities, structures and chemical modifications of individual proteins and protein complexes.

- The dynamics of cellular protein composition and well as the localization and assembly of multiprotein complexes.

- The properties and dynamics of biological membranes

- The interaction of cells with each other and with the extracellular environment.

Work in these topical areas can utilize current EMSL capabilities as well as extend capabilities into new technical areas. For example, a full understanding of the structure, function, and dynamics of multiprotein complexes and a detailed metabolite profiling of many cells will require extending current EMSL capabilities in high-throughput mass spectrometry and NMR. Work is currently underway to enhance EMSL capabilities in the analysis of microbial membranes and cellular interactions by the development of new techniques, such as cryo-TEM, and multimodal and multispectral microscopy. Efforts are also underway to integrate and analyze the large amounts of data generated by EMSL instruments through an integrated data management system. Proposals to use these developing capabilities are particularly encouraged. 


\title{
De novo Sequencing of Unique Sequence Tags for Discovery of Post- Translational Modifications of Proteins
}

\author{
Y Shen, ${ }^{(a)}$ N Tolic, ${ }^{(b)}$ KK Hixson, ${ }^{(b)}$ So Purvine, ${ }^{(b)}$ GA Anderson, ${ }^{(a)}$ and RD Smith ${ }^{(a)}$ \\ (a) Pacific Northwest National Laboratory, Richland, Washington \\ (b) EMSL, Richland, Washington
}

EMSL researchers and users have developed a unique strategy that allows for unambiguous and highly confident protein identifications, which can help better identify changes that signal disease, potentially improving techniques for early disease detection and monitoring changes in environmentally important organisms.

To improve proteomics studies of heart disease, cancer, neurodegenerative diseases, and diabetes, EMSL researchers and users developed a novel strategy that expands protein identifications beyond those predicted from genomic sequences. Their approach combines de novo sequencing and the UStag approach to discover post-translational modifications in proteins. De novo sequencing derives a peptide sequence without the help of a database of known protein sequences. The UStag approach uses a peptide sequence to unambiguously and uniquely identify a single protein.

The combined de novo - UStag approach, funded in part by EMSL's 2007 Intramural Research Program, allows scientists to discover protein posttranslational modifications, including complex multiple unknown/unexpected modifications on single protein sequences, and discover sequence mutations and genome-predicted database sequence errors. This research and other discoveries are advancing EMSL's goal of being able to predict biological functions from molecular and chemical data.

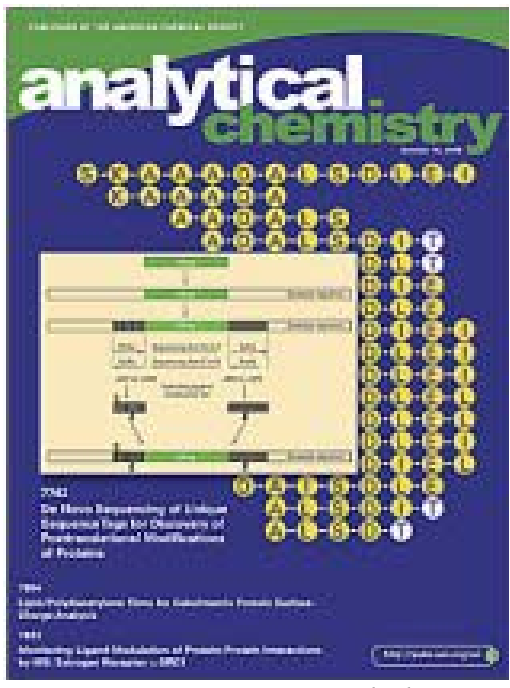

Figure 1. A new approach that characterizes previously unknown protein modifications was featured on the cover of Analytical Chemistry.

This research, which was funded by EMSL's Intramural Research Program, DOE's Office of Biological and Environmental Research, and the National Institutes of Health's National Center for Research Resources, was featured on the cover of the October 15, 2008, issue of Analytical Chemistry (Figure 1).

\section{Citation}

Shen Y, N Tolic, KK Hixson, SO Purvine, GA Anderson, and RD Smith. 2008. "De novo Sequencing of Unique Sequence Tags for Discovery of Post-Translational Modifications of Proteins." Analytical Chemistry 80(20):7742-7754. 


\title{
The Effects of Aging on the Luminescence of P5EG-Coated Water- Soluble ZnO Nanoparticles Solutions
}

\author{
BK Woo, ${ }^{(a)}$ W Chen, ${ }^{(a)}$ AG Joly, ${ }^{(b)}$ and R Sammynaiken ${ }^{(c)}$ \\ (a) University of Texas at Arlington, Arlington, Texas \\ (b) Pacific Northwest National Laboratory, Richland, Washington \\ (c) University of Saskatchewan, Saskatoon, Saskatchewan, Canada
}

A team of EMSL users has conducted basic research that is furthering the understanding of the interactions of nanoparticles and their surroundings, and is providing insights into biomedical uses of nanoparticles, such as treating malignant tumors.

EMSL users from the University of Texas at Arlington, Pacific Northwest National Laboratory, and the University of Saskatchewan used EMSL expertise and instrumentation at to characterize the light-emitting properties of coated, water-soluble zinc oxide nanoparticles potentially useful in killing cancer cells. These particles could be targeted at malignant cells, followed by administration of X-rays that would cause the particles to glow. The light would activate a photosensitive chemical, also on the particles, that, in turn, would create energized oxygen that could destroy nearby cancer cells.

The team found that the particles' coating of polyethylene glycol biscarboxymethyl increased the particles luminescence intensity over a period of 50+ days (Figure 1). The resulting nanoparticles displayed increased luminosity relative to the uncoated water-soluble particles.

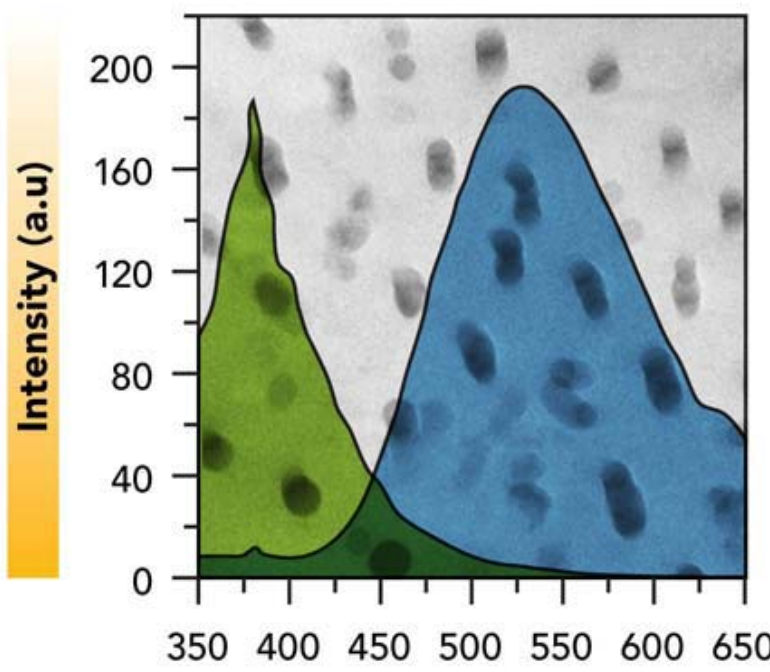

\section{Wavelength (nm)}

Figure 1. The coating on the water-soluble zinc oxide particles allows them to deliver bright light for more than 7 weeks.

The uncoated particles luminesce because of defects on the surface. The coating fills in these defects, allowing the particles to rely on a different method of luminescence. This pathway is known as bandedge states. This characterization was done using the laser-based fluorescence spectrometer facilities at EMSL. The light from these particles can be used as part of an innovative treatment for deep tissue cancers, such as breast cancer.

This research, supported by the University of Texas at Arlington, National Science Foundation, Department of Homeland Security, and Department of Defense, was featured in the Journal of Physical Chemistry $C$.

\section{Citation}

Woo BK, W Chen, AG Joly, and R Sammynaiken. 2008. "The Effects of Aging on the Luminescence of P5EG-Coated Water-Soluble ZnO Nanoparticles Solutions.” Journal of Physical Chemistry C 112(37):14292-14296. doi: 10.1021/jp803649k 


\title{
Automated Gain Control Ion Funnel Trap for Orthogonal Time-of- Flight Mass Spectrometry
}

\author{
YM Ibrahim, ${ }^{\text {(a) } M E ~ B e l o v, ~}{ }^{\text {(a) }}$ AV Liyu, ${ }^{\text {(b) }}$ and RD Smith ${ }^{(a)}$ \\ (a) Pacific Northwest National Laboratory, Richland, Washington \\ (b) EMSL, Richland, Washington
}

EMSL users have developed improved mass spectrometry (MS) tools that detect peptides with ten times more sensitivity. Diverse applications of this new technology to environmental and biological research include early disease detection, identification of new pharmaceutical targets, and testing for agents that may threaten homeland security.

A research team comprised of scientists from Pacific Northwest National Laboratory and EMSL upped the ante for mass spectrometry (MS) by adding an electrodynamic ion funnel trap (IFT) with automated gain control (AGC) to a conventional time-of-flight (TOF) MS system, allowing for optimized ion control. The first step to MS analysis is to generate ions by electrospray ionization; ions are then fed to a TOF MS for mass and charge analysis. Because the MS is typically coupled with another analytical tool (reversed-phase capillary liquid chromatography), ions are not generated at

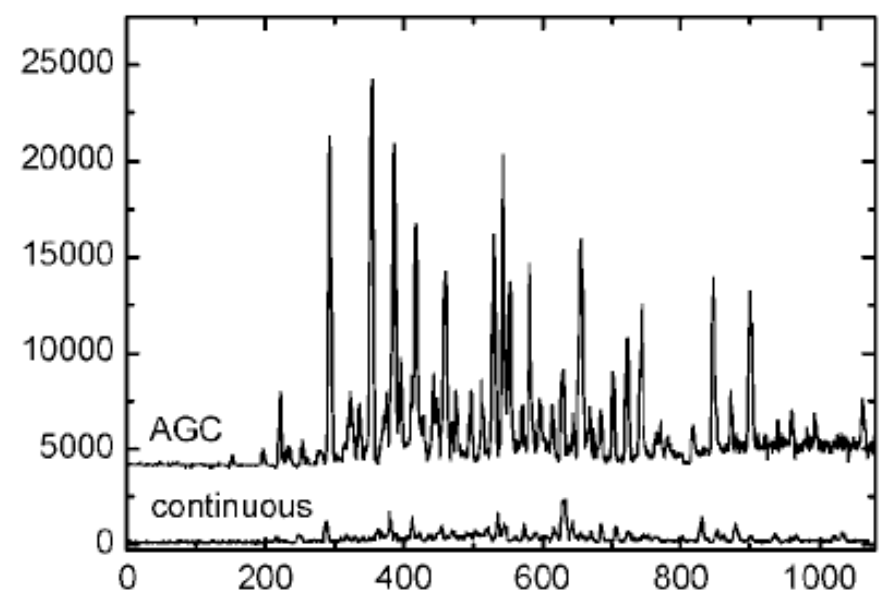

Figure 1. Peak intensity for S.oneidensis MS measurements is greater in the AGC mode, demonstrating higher sensitivity of detection.

a constant rate, and the concentrations of ions passed to a TOF MS from an ion trap may exceed the TOF MS optimal detection range. By adding IFT with AGC to a conventional TOF MS system, the team was able to control the concentration of ions, minimizing the possibility of TOF MS detector saturation. In addition, ion accumulation in the IFT with AGC drastically improved signal-to-noise ratios for lowconcentration analytes as compared to conventional TOF MS analysis.

To test the effectiveness of the IFT AGC modification, the team analyzed the proteome of Shewanella oneidensis, a bacterium that can chemically modify and neutralize toxic metals without apparent harm to itself. Compared to conventional techniques, the team's new and improved MS tool gave rise to ten times more sensitivity and greater mass measurement accuracy for analysis of low-concentration samples, yielding five times as many $S$. oneidensis unique peptides with the AGC mode (383 peptides) than with the conventional continuous mode of operation (73 peptides) (Figure 1).

The research is supported by the DOE Office of Biological and Environmental Research's Genomes:GtL Program and the National Institutes of Health's National Center for Research Resources and National Institute of Allergy and Infectious Diseases. It was featured in Analytical Chemistry.

\section{Citation}

Ibrahim YM, ME Belov, AV Liyu, and RD Smith. 2008. "Automated Gain Control Ion Funnel Trap for Orthogonal Time-of-Flight Mass Spectrometry.” Analytical Chemistry 80(14):5367-5376. 


\title{
Transport Functions Dominate the SAR11 Metaproteome at Low Nutrient Extremes in the Sargasso Sea
}

\author{
SM Sowell, ${ }^{(a)}$ LF Wilhelm, ${ }^{(a)} A D$ Norbeck, ${ }^{(b)}$ MS Lipton, ${ }^{(b)}$ CD Nicora, ${ }^{(b)}$ DF Barofsky, ${ }^{(a)}$ \\ CA Carlson, ${ }^{(c)}$ RD Smith, ${ }^{(b)}$ and SJ Giovanonni ${ }^{(a)}$ \\ (a) Oregon State University, Corvallis, Oregon \\ (b) Pacific Northwest National Laboratory, Richland, Washington \\ (c) University of California, Santa Barbara, California
}

EMSL proteomics resources were critical to pioneering research in which scientists, for the first time, measured protein expression in microbial communities from the Sargasso Sea. The insight afforded by this research into oceanic microbial communities is important because such bacteria heavily influence biogeochemical cycles, affecting the concentrations of elements such as carbon-and therefore the greenhouse gas, carbon dioxide - in the Earth's air, water, and soil.

The team of EMSL users from Oregon State University, Pacific Northwest National Laboratory, and the University of California conducted these experiments using a new proteomics technique, metaproteomics, which allows identification of proteins in mixed cultures even without a complete genome to help. Metaproteomics analyses revealed that the lion's share of peptides detected in Sargasso Sea microbial communities were unique to Prochlorococcus, Synechococcus, or SAR 11 (Peligobacter ubique) (Figure 1), which is one of the most abundant organisms on earth. Further, these bacteria have adapted to their harsh environment, in which they are subjected to damage by light and oxidative stress, by expressing an abundance of transport proteins for nutrient uptake. This

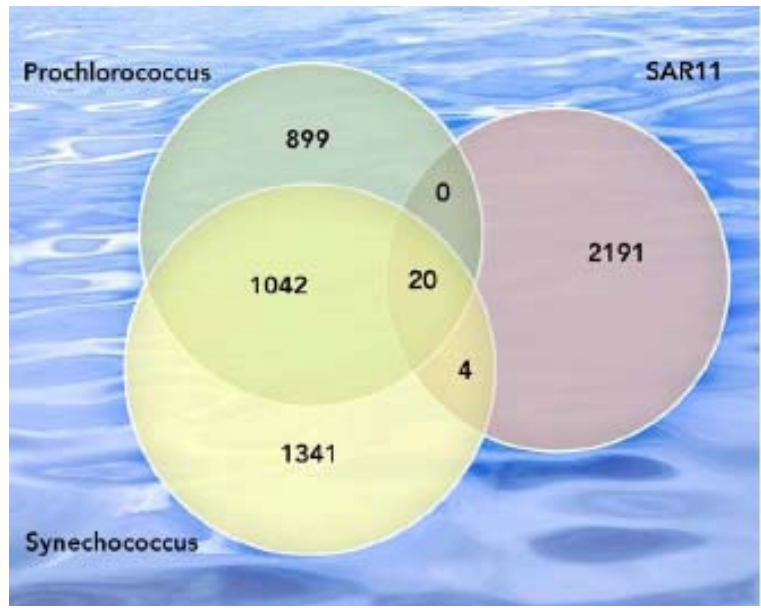

Figure 1. A large proportion of the peptides detected in samples from the Sargasso Sea were unique to Prochlorococcus, Synechococcus, or SAR 11. mechanism allows them to sequester the very limited essential nutrients needed for their survival from the waters that surround them.

The team's work applies new proteomics methods to complex microbial community research and, furthermore, crosses the boundaries of many disciplines, connecting proteomics, microbiology, and climate research. In addition, these studies support EMSL's goal to predict biological functions from molecular and chemical data. In addition, understanding how microbial communities use transport proteins to adapt to seasonal changes and fluctuations in the levels of atmospheric carbon yields insight into global carbon cycling and could help in the design of strategies to address global warming.

The research, supported by a Marine Microbiology Initiative Investigator Award from the Gordon and Betty Moore Foundation and, in part, by the DOE Office of Biological and Environmental Research, was published in International Society for Microbial Ecology (ISME) Journal. 


\title{
Citation
}

Sowell SM, LF Wilhelm, AD Norbeck, MS Lipton, CD Nicora, DF Barofsky, CA Carlson, RD Smith, and SJ Giovanonni. 2009. "Transport Functions Dominate the SAR11 Metaproteome at Low Nutrient Extremes in the Sargasso Sea." International Society for Microbial Ecology (ISME) Journal 3:93-105. DOI:10.1038/ismej.2008.83

\section{MR Imaging of Apparent ${ }^{3} \mathrm{He}$ Gas Transport in Narrow Pipes and Rodent Airways}

\author{
KR Minard, ${ }^{(a)}$ RE Jacob, ${ }^{(a)}$ G Laicher, $^{(b)}$ DR Einstein, ${ }^{(a)}$ AP Kuprat, ${ }^{(a)}$ and RA Corley ${ }^{(a)}$ \\ (a) Pacific Northwest National Laboratory, Richland, Washington \\ (b) University of Utah, Salt Lake City, Utah
}

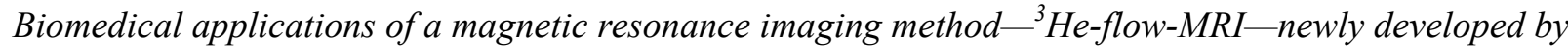
users at EMSL range from improving inhaled drug delivery to monitoring therapeutic response in patients with breathing disorders like asthma or Chronic Obstructive Pulmonary Disease. The ability to measure alterations in regional lung ventilation also provides a unique opportunity for assessing the subtle effects of inhaled pollutants, and for improving assessment of their potential health risks.

Airflow patterns in the lung not only determine how well you breathe but also how inhaled materials like airborne pollutants or aerosolized drugs are distributed inside the human body. At EMSL, users from Pacific Northwest National Laboratory and the University of Utah have pioneered a new magnetic resonance imaging method for visualizing inhaled airflow patterns. The method uses hyperpolarized ${ }^{3} \mathrm{He}$ gas as an inert tracer for visualizing inhaled air speed and direction at each location within the complex, threedimensional airways of pulmonary anatomy.

Published results highlighted on the cover of the Journal of Magnetic Resonance (Figure 1) reveal a common aerodynamic phenomenon known as air streaming. This was observed as a thin layer of high-speed gas localized along the outside radius of curvature in the trachea. The results also show, for the first time, how air speed is reduced as flowing gas branches along different paths to fill each of the rat's five lung lobes. This was seen as a transition from high gas speed to a slower flow rate.

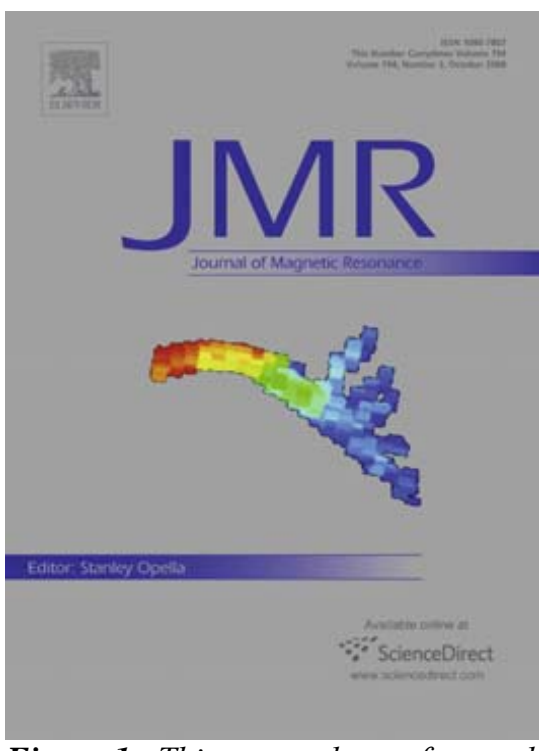

Figure 1. This research was featured on the cover of Journal of Magnetic Resonance.

The new system is being used in conjunction with its advanced computing resources to develop and test state-of-the art computer models of inhaled airflow. These models are important not only for predicting where inhaled materials are deposited in the lung, but also for understanding how their fate ultimately affects human health. This work is part of EMSL's ongoing efforts to predict biological functions from molecular and chemical data. EMSL users are also exploiting the system to help understand the risks associated with common pesticides of interest to the Environmental Protection Agency. They have also begun to exploit ${ }^{3} \mathrm{He}$-flow-MRI for visualizing electrochemistry in operating fuel cells as part of efforts to improve operating efficiency and reliability. 
The research is supported by the National Institutes of Health's National Heart, Lung and Blood Institute.

\title{
Citation
}

Minard KR, RE Jacob, G Laicher, DR Einstein, AP Kuprat, and RA Corley. 2008. "MR Imaging of Apparent ${ }^{3} \mathrm{He}$ Gas Transport in Narrow Pipes and Rodent Airways." Journal of Magnetic Resonance 194(2):182-191.

\section{A Solution NMR Investigation into the Early Events of Amelogenin Nanosphere Self-Assembly Initiated with Sodium Chloride or Calcium Chloride}

\author{
GW Buchko, ${ }^{(a)}$ BJ Tarasevich, ${ }^{(a)}$ JG Bekhazi, ${ }^{(a)}$ ML Snead, ${ }^{(b)}$ and WJ Shaw ${ }^{(a)}$ \\ (a) Pacific Northwest National Laboratory, Richland, Washington \\ (b) University of Southern California, Los Angeles, California
}

New studies performed using EMSL NMR capabilities reveal how the hardest material in the human body, tooth enamel, starts to form at the molecular level. Such studies give insights into the nature of orthodontic diseases such as amelogenesis imperfecta, which results in defective enamel formation and has been tied to N-terminus defects in amelogenin.

EMSL users from Pacific Northwest National Laboratory and University of Southern California used EMSL nuclear magnetic resonance resources to study the mechanisms behind self assembly of the protein, amelogenin, into nanospheres - an initial step in building enamel. Amelogenin provides a matrix upon which another protein, hydroxyapatite, builds a strong three-dimensional structure to form enamel. Using solution-state NMR spectroscopy and dynamic light scattering, the research team followed the initial steps of amelogenin matrix formation in solutions containing different levels of salt, a variable that triggers protein self-association. Two salts were tested (sodium chloride and calcium chloride), and both yielded similar step-wise results for amelogenin interaction (Figure 1). At low pH with no salt in solution, amelogenin exists as individual protein molecules, (monomers). Upon adding salt to the solution, pairs of amelogenin monomers start to interact at their N-termini to form dimers. As the salt concentration is increased further, the interaction between pairs of monomers grows stronger as the association extends to include interactions between the C-termini. Dimer formation is the first step in amelogenin self-assembly; the full self-assembly process yields amelogenin nanospheres.

The team's research provides a better understanding of the formation of nanospheres thought to have an essential functional role in enamel formation. Their work also offers insight into the nature of orthodontic diseases, such as amelogenesis imperfecta, which results in defective enamel formation. Further, this work supports EMSL's goal to predict biological functions from molecular and chemical data.

The research, supported by the National Institutes of Health's National Institute of Dental and Craniofacial Research, was published in Biochemistry. 

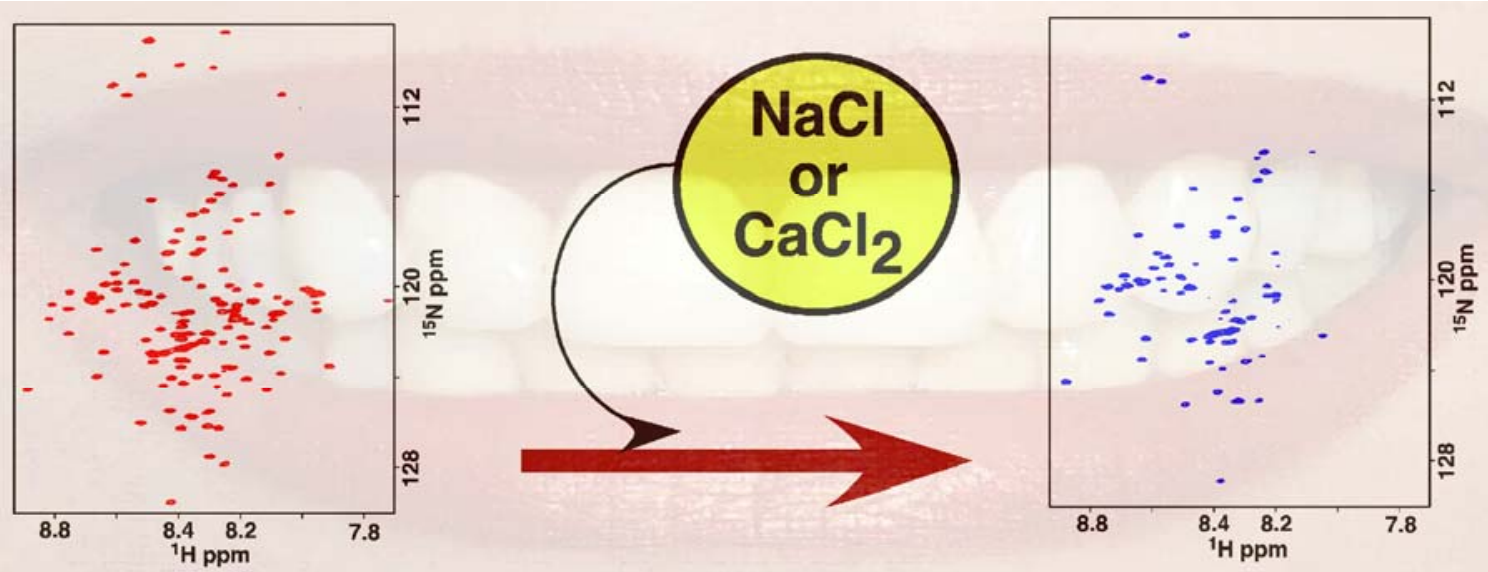

Figure 1. Self-assembly of amelogenin was monitored by following changes to the NMR spectra ( $1 \mathrm{H}-15 \mathrm{~N} H S Q C)$ of amelogenin without salt (left, monomers) and after the addition of salt (right, dimers and larger).

\section{Citation}

Buchko GW, BJ Tarasevich, JG Bekhazi, ML Snead, and WJ Shaw. 2008. "A Solution NMR Investigation into the Early Events of Amelogenin Nanosphere Self-Assembly Initiated with Sodium Chloride or Calcium Chloride." Biochemistry 47(50):13215-13222. doi: 10.1021/bi8018288.

\section{Enhanced Detection of Low-Abundant Human Plasma Proteins using a Tandem IgY12-SuperMix Immunoaffinity Separation Strategy}

\section{W Qian, ${ }^{(a)}$ DT Kaleta, ${ }^{(a)}$ BO Petritis, ${ }^{(a)}$ H Jiang, ${ }^{(a)} T$ Liu, ${ }^{(a)} X Z_{\text {Zhang, }}{ }^{(a)} H M$ Mottaz, ${ }^{(b)}$ SM Varnum, ${ }^{\text {(a) }}$ DG Camp II, ${ }^{\text {(a) }}$ L Huang, ${ }^{\text {(c) }}$ X Fang, ${ }^{(d)}$ W Zhang, ${ }^{(d)}$ and RD Smith ${ }^{(a)}$}

(a) Pacific Northwest National Laboratory, Richland, Washington

(b) EMSL, Richland, Washington

(c) GenWay Biotech, Inc., San Diego, California

(d) Acrotics Corp, San Diego, California

EMSL users have designed a more sensitive protein separations technology, the tandem IgY12-SuperMix, that allows scientists to detect cytokines and other molecules of interest at the low nanogram-permilliliter to sub-nanogram-per-milliliter range in the presence of other proteins that are more than 8 orders of magnitude higher in abundance. The system will help accelerate the discovery of novel biomarkers for improved diagnosis and prognosis of human diseases such as cancer, which will, in turn, impact the biomedical practice and human health.

Using liquid chromatography, mass spectrometry, and other instruments at EMSL, scientists from Pacific Northwest National Laboratory, EMSL, and GenWay Biotech, Inc. designed a new protein separations system - the tandem IgY12-SuperMix - that enhances the detection of hard-to-find proteins in human plasma (Figure 1). Using this new approach with the proteomics technologies developed at PNNL, the scientists removed the $\sim 60$ most abundant proteins from plasma samples. With these proteins removed, the scientists detected numerous low-abundance proteins. This technology makes the discovery of lowabundance disease biomarkers more feasible. The team is developing methodologies to detect breast cancer and other disease state biomarkers. 
Funded by the National Institutes of Health's National Center for Research Resources, the National Institute of General Medical Sciences, the Entertainment Industry Foundation (EIF) and the EIF Women's Cancer Research Fund, this work is part of EMSL's ongoing efforts to predict biological functions from molecular and chemical data. The research was published in Molecular \& Cellular Proteomics. MCP.

\title{
Citation
}

Qian W, DT Kaleta, BO Petritis, H Jiang, T Liu, X Zhang, HM Mottaz, SM Varnum, DG Camp II, L Huang, X Fang, W Zhang, and RD Smith. 2008. "Enhanced Detection of Low-Abundant Human Plasma Proteins using a Tandem IgY12-SuperMix Immunoaffinity Separation Strategy." Molecular \& Cellular Proteomics. MCP 7(10):1963-1973.

\section{Global Systems-Level Analysis of Hfq and SmpB Deletion Mutants in Salmonella: Implications for Virulence and Global Protein Translation}

\author{
C Ansong, ${ }^{(\text {a) }} \mathrm{H}$ Yoon, ${ }^{(b)} \mathrm{S}$ Porwollik, ${ }^{(c)} \mathrm{H}$ Mottaz-Brewer, ${ }^{(d)}$ BO Petritis, ${ }^{(a)} \mathrm{N}$ Jaitly, ${ }^{(a)}$ \\ JN Adkins, ${ }^{(a)} M$ McClelland, ${ }^{(c)}$ F Heffron, ${ }^{(b)}$ and RD Smith ${ }^{(a)}$ \\ (a) Pacific Northwest National Laboratory, Richland, Washington \\ (b) Oregon Health and Sciences University, Portland, Oregon \\ (c) The Sidney Kimmel Cancer Center, San Diego, California \\ (d) EMSL, Richland, Washington
}

One of the most complete analyses of global post-transcriptional regulatory mechanisms in any organism indicates that post-transcriptional regulation plays an unexpectedly prominent role in the bacteria Salmonella's ability to infect its host. Post-transcriptional regulation is the control of protein synthesis by genes after RNA synthesis has begun. 
The analyses, performed by scientists from

Pacific Northwest

National Laboratory, Oregon Health Sciences University and The Sidney Kimmel Cancer Center were described in the online journal PLoS One. The team investigated the proteome of Salmonella Typhimurium mutant strains lacking specific RNA-binding proteins that mediate the translation of RNA into protein (posttranscriptional control)

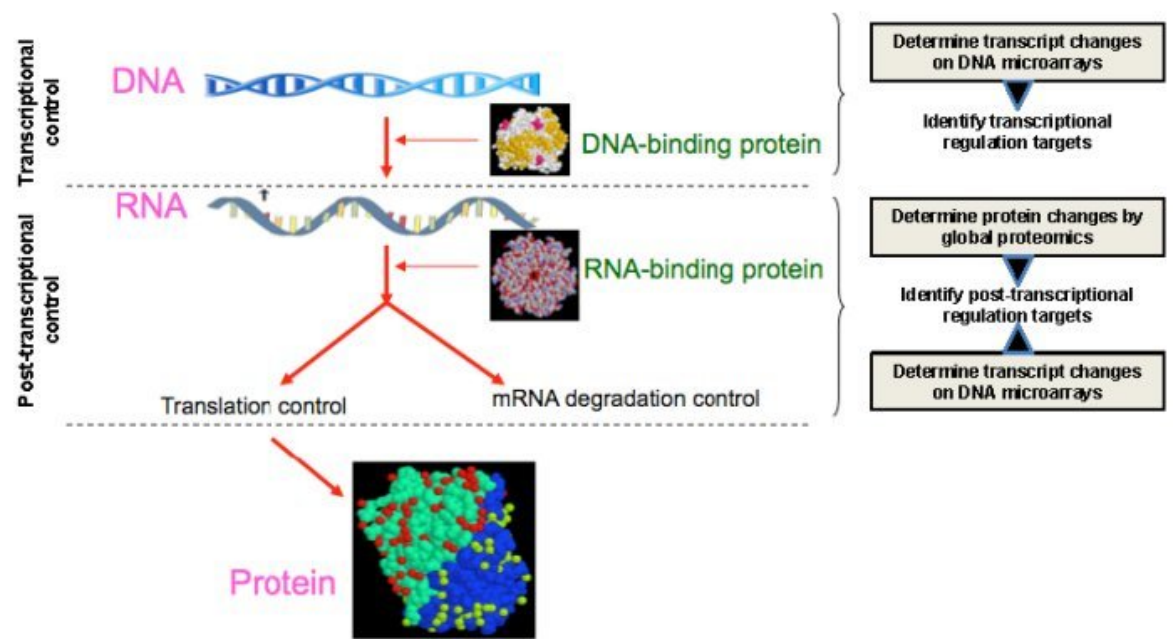

Figure 1. Sample matched proteomics-transcriptomics measurement capabilities now make it possible to quantitatively characterize global post-transcriptional control mechanisms. RNA-binding proteins mediate post-transcriptional control. Quantitative global proteomics measurements determine the protein-dependent changes in protein expression and sample-matched transcriptomics analysis reveals if the change in protein expression is mediated post-transcriptionally. and the parent strain. Specifically, the team investigated the Salmonella RNA-binding virulence proteins Hfq and SmpB. The team then compared the proteomics data to sample-matched transcriptomics data to characterize the global control of gene expression at the post-transcriptional level (Figure 1). "Samplematched" means that RNA for transcriptomics analysis and protein for proteomics analysis were prepared from the same sample.

The comparison revealed that a relatively high percentage of all the annotated Salmonella genes ( $\geq 20 \%)$ are regulated post-transcriptionally. The extent of post-transcriptional regulation observed is much greater than previously thought with profound effects in all stages of Salmonella's life cycle. These include known and novel virulence factors.

Hfq and SmpB are proteins essential for virulence in a wide range of pathogenic bacteria including Salmonella, a leading cause of foodborne illness. Salmonella Typhimurium was the bacterium of interest in the recall of peanut butter crackers in 2008 and 2009. Understanding how Hfq and SmpB impact Salmonella's virulence is important for protecting human health and agriculture.

While the role that DNA-binding proteins play in regulating gene expression at the transcriptional level is well studied, how RNA-binding proteins control gene expression at the post-transcriptional level is less clear. The team's work provides one of the first quantitative global analyses of the role RNA-binding proteins play in regulating gene expression and makes a significant contribution to knowledge of this regulatory process.

The researchers used capillary liquid chromatography-mass spectrometry analyses to analyze the proteomes of Salmonella mutant strains lacking specific RNA-binding proteins that mediate the translation of RNA into protein (post-transcriptional control) and the parent strain grown under four different conditions. They then applied the accurate mass and time tag strategy developed at PNNL to quantitate proteins in the samples. They employed whole genome microarray analysis to perform samplematched transcriptome analyses. Bioinformatics approaches were then used to compare the proteomics 
data to sample-matched transcriptomics data to identify targets of post-transcriptional control and provide quantitative information on changes in the expression pattern of targets at the level of translation.

The genomes of most living organisms including all animals, plants and bacteria encode a large number of RNA-binding proteins with increasing evidence pointing to their extensive involvement in posttranscriptional regulatory events. The work described here has established the framework for the future systematic characterization of the global program and molecular mechanisms of post-transcriptional regulation of gene expression in any genetically tractable organism.

This research was supported by the National Institute of Allergy and Infectious Diseases, and the National Center for Research Resources.

\section{Citation}

Ansong C, H Yoon, S Porwollik, H Mottaz-Brewer, BO Petritis, N Jaitly, JN Adkins, M McClelland, F Heffron, and RD Smith. 2009. "Global Systems-Level Analysis of Hfq and SmpB Deletion Mutants in Salmonella: Implications for Virulence and Global Protein Translation.” PLoS ONE 4(3):e4809.

\section{A Targeted Releasable Affinity Probe (TRAP) for in vivo Photo- Crosslinking} $P$ Yan, ${ }^{(a)} T$ Wang,,$^{(b)}$ GJ Newton, ${ }^{(c)}$ TV Knyushko, ${ }^{(d)}$ Y Xiong, ${ }^{(b)}$ DJ Bigelow, ${ }^{(b)}$ TC Squier, $^{(b)}$
and M Uljana Mayer

(a) Novozymes, Inc., Davis, California

(b) Pacific Northwest National Laboratory, Richland, Washington

(c) The University of Tokyo, Tokyo, Japan

(d) Harris IT Services, Bethesda, Maryland

DNA might be the blueprint for living things, but proteins are the builders. Researchers trying to understand how and which proteins work together have developed a new cross-linking tool that is small and unobtrusive enough to use in live cells. Using the new tool, the scientists have discovered new details about a well-studied complex of proteins known as RNA polymerase. The results suggest the method might uncover collaborations between proteins that are too brief for other techniques to pinpoint.

Proteins are the workhorses in an organism's cells. Whole fields of research are dedicated to teasing out which proteins work together to make cells function. For example, drug researchers seek chemicals that disrupt or otherwise change how proteins interact to combat diseases; environmental scientists need to understand how proteins collaborate in ecosystems to make them thrive or fail.

To learn about protein networks, scientists start with a familiar one and use it as bait to find others that work alongside it. To pin down the collaborators, researchers make physical connections between old and new proteins with chemicals called crosslinkers. The sticky crosslinkers will only connect proteins close enough to work together, the thinking goes. But most crosslinkers are too large to squeeze into living cells, are harmful to cells, or link proteins that are neighbors but not coworkers.

To address these issues, the research team developed a crosslinking method that uses small crosslinkers whose stickiness can be carefully controlled. To find coworkers of a protein of interest, they built a tiny molecule called a tag into the initial protein. They then add a small molecule called TRAP to the living 
cell, which finds and fits into the tag like two pieces in a puzzle. TRAP waves around, bumping into nearby proteins. The scientists control TRAP with a flash of light, causing it to stick to coworkers it bumps into. The researchers then identify the new "TRAPped" proteins in subsequent analyses (Figure 1).

To demonstrate how well this method works, the team tested it out on RNA polymerase, a well-studied machine in cells. The polymerase is made up of many proteins that cooperate to translate DNA. One of the polymerase proteins has a tail that is known to touch the DNA and some helper proteins just before the polymerase starts translating. No one knew if this tail -- also known as the C-terminus of the alpha subunit -touches anything else in the core of the RNA polymerase complex.

The team engineered a tag in the $\mathrm{C}$ terminus and cultured bacteria with the tagged RNA polymerase. After adding

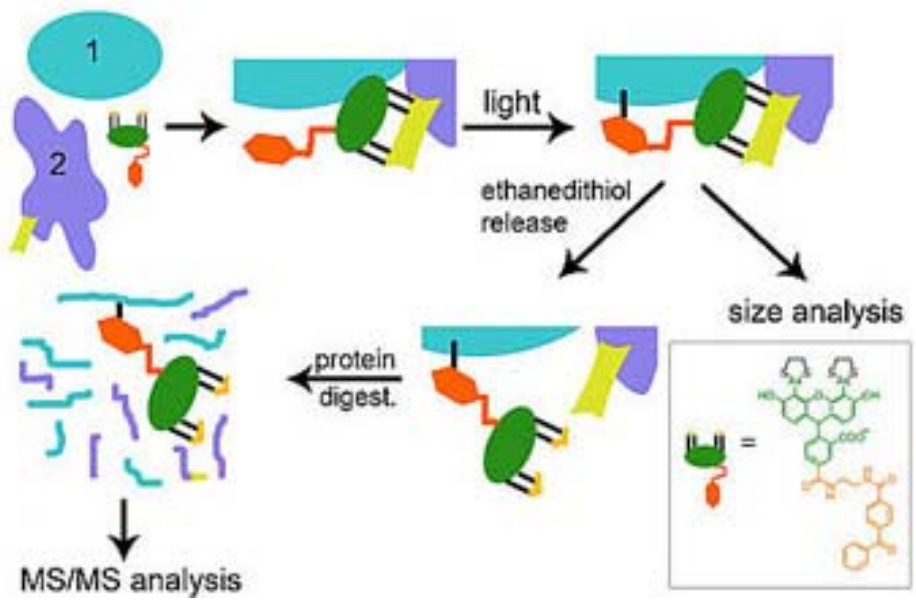

Figure 1. TRAP (in green, orange and yellow) binds to a tag on known protein (\#2). Light crosslinks TRAP's benzophenone to mystery protein (\#1). Subsequent biochemical analysis reveals clues to unknown protein (\#1).

TRAP to the cells and giving it time to find the C-terminus tag, the team shined a light on the cultures.

The team then identified the proteins marked with TRAP using instruments in EMSL. They found that the tagged protein, as expected, interacts with many other proteins, for example previously identified helper proteins, so-called transcription factors. But they also found it on another core protein called the beta subunit, suggesting the tail of the alpha subunit makes contact with the beta subunit as it plugs along. This interaction had never been seen before.

The team reported their results in the journal ChemBioChem. The tag in their unique method is made up of a "tetracysteine motif" -- two pairs of the amino acid cysteine separated by two other amino acids that doesn't interfere with the normal function of the protein of interest. TRAP includes a small "biarsenical" probe, which fluoresces so the team can find the proteins to which it has become attached. TRAP can also be easily unlinked from the tag with a simple biochemical treatment, allowing researchers to piece out the coworker from their original protein of interest.

The team also tested the method on other proteins, such as those found in young muscle cells. Mayer said they will use the method in the future to understand how environmental conditions affect how proteins work together in large networks.

The research is funded by DOE's Office of Biological and Environmental Research.

\section{Citation}

Yan P, T Wang, GJ Newton, TV Knyushko, Y Xiong, DJ Bigelow, TC Squier, and MU Mayer. 2009. "A Targeted Releasable Affinity Probe (TRAP) for in vivo Photo-Crosslinking." ChemBioChem 10:1507 1518. 


\title{
An Integrated Top-Down and Bottom-Up Strategy for Broadly Characterizing Protein Isoforms and Modifications
}

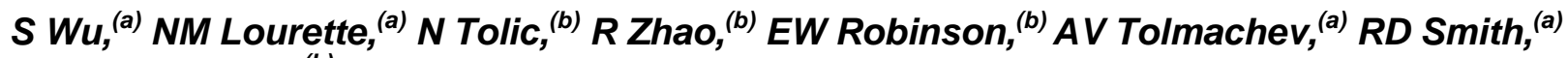 \\ and L Pasa-Tolic ${ }^{(b)}$ \\ (a) Pacific Northwest National Laboratory, Richland, Washington \\ (b) EMSL, Richland, Washington
}

An integrated top-down bottom-up proteomics strategy, as developed by a team of EMSL users and researchers, could play a critical role in identifying and characterizing biomarkers of cancer, cardiovascular disease, neurological disease, diabetes, and autism.

Researchers at Pacific

Northwest National Laboratory

and EMSL used EMSL

resources to develop an

innovative approach that

integrates two fundamental

proteomic strategies for protein

identification and

characterization by mass

spectrometry (Figure 1). The

approach overcomes the

limitations of the traditional

top-down and bottom-up

strategies, allowing for high-

throughput analysis of protein

isoforms and genetic variance

through amino acid

modifications, such as

acetylation and

phosphorylation.

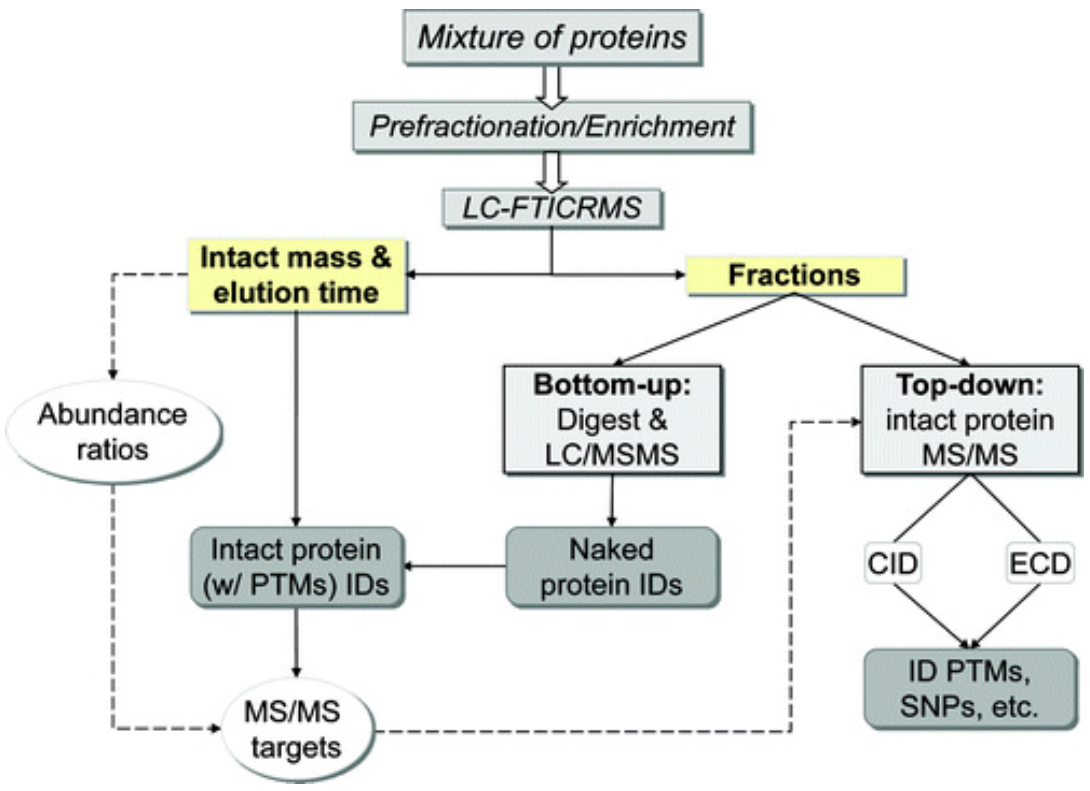

Figure 1. Integrated top-down and bottom-up strategy for characterizing protein isoforms and modifications

Bottom-up sequencing is the analysis of trypsin-digested proteins and is the basis for contemporary proteomics. However, researchers are limited by the bottom-up approach because complete sequence coverage of proteins is rarely achieved. Top-down analysis involves direct analysis of intact proteins without digestion. But it suffers from limited throughput and has a low success rate when used with online liquid chromatography-tandem mass spectrometry and online fraction collection. By combining top-down and bottom-up approaches, scientists can achieve more precise identification and characterization of protein isoforms and combinatorial post-translational modifications. The researchers demonstrated their integrated strategy by coupling reversed-phase liquid chromatography with a 12-Tesla Fourier transform ion cyclotron resonance mass spectrometer and online fraction collection. They identified modified proteins by applying intact protein masses with "bare" proteins from the bottom-up analysis of the collected fractions. Integrated proteomics opens up new areas in the profiling of potential biomarkers that may be used to diagnose disease and is a more comprehensive approach to protein identification and profiling, allowing for high-throughput characterization of intact proteins and their modifications. 
This work was supported by the National Center for Research Resources, the National Institute of Allergy and Infectious Diseases, the National Institute of General Medical Sciences, and DOE's Office of Biological and Environmental Research. It was featured in Journal of Proteome Research.

\title{
Citation
}

Wu Si, M Lourette, N Tolic, R Zhao, E Robinson, AV Tolmachev, RD Smith, and L Pasa-Tolic. 2009. "An Integrated Top-Down and Bottom-Up Strategy for Broadly Characterizing Protein Isoforms and Modifications.” Journal of Proteome Research 8(3):1347-1357. DOI: 10.1021/pr800720d

\section{Platelet Proteome Changes Associated with Diabetes and during Platelet Storage for Transfusion}

\author{
DL Springer, ${ }^{(a)} J_{H}$ Miller, ${ }^{(b)} S L$ Spinelli, ${ }^{(c)}$ L Pasa-Tolic, ${ }^{(d)}$ SO Purvine, ${ }^{(d)} D S$ Daly,,$^{(a)} R C$ \\ Zangar, ${ }^{(a)} S$ Jin, ${ }^{(b)} \mathrm{N}$ Blumberg, ${ }^{(c)} \mathrm{CW}$ Francis, ${ }^{(c)} \mathrm{MB}$ Taubman, ${ }^{(c)}$ AE Casey, ${ }^{(c)}$ SD Wittlin, ${ }^{(c)}$ \\ and RP Phipps ${ }^{(c)}$ \\ (a) Pacific Northwest National Laboratory, Richland, Washington \\ (b) Washington State University-Tri-Cities, Richland, Washington \\ (c) University of Rochester School of Medicine and Dentistry, Rochester, New York \\ (d) EMSL, Richland, Washington
}

About 18 million people in the United States have type 2 diabetes, and the disease is spreading with the epidemic of obesity. Amid emerging concerns that blood platelets donated for transfusion by individuals with Type 2 diabetes may be unsafe, scientists are reporting the first detailed identification and quantification of proteins in the platelets from diabetic donors. The study could lead to screening tests to detect and monitor these "high risk platelet preparations," say EMSL users from Pacific Northwest National Laboratory, Washington State University-Tri-Cities, and the University of Rochester. Their study appeared in the May issue of the Journal of Proteome Research.

Thousands of patients receive potentially lifesaving transfusions of platelets each year to treat bleeding from trauma and for a wide range of medical conditions. Scientists have known that activated platelets in the blood of diabetics may predispose these individuals to heart disease. This led to the concern that platelets from these individuals stored for transfusion may be less effective and even unsafe. However, scientists know little about how diabetic platelets differ from those of healthy people.

In this study, Dr. David Springer, a biochemist at PNNL, and colleagues identified 122 proteins whose abundances differ in the platelets of individuals with diabetes compared to the platelets of non-diabetics. They also found that freshly collected platelets from diabetics show almost as many changes (more than 100) in protein abundance as healthy donor platelets stored for up to 5 days. These findings could lead to new tests for detecting and monitoring abnormal platelets to improve the outcome of blood transfusions from both diabetic and healthy individuals.

The researchers used EMSL mass spectrometry-based proteomics on platelets from healthy and type 2 diabetics collected at the University of Rochester Blood Bank. They also investigated the role of transfusion storage, including diabetes effects on the platelet proteome. Their approach enabled highly sensitive detection of a variety of molecular species in platelets from diabetics.

This work was supported by the National Institutes of Health. 


\title{
Citation
}

Springer DL, JH Miller, SL Spinelli, L Paša-Tolic, SO Purvine, DS Daly, RC Zangar, S Jin, N Blumberg, CW Francis, MB Taubman, AE Casey, SD Wittlin, and RP Phipps. 2009. "Platelet Proteome Changes Associated with Diabetes and during Platelet Storage for Transfusion " Journal of Proteome Research $8(5): 2261-2272$.

\section{Engineering an Ultra-Stable Affinity Reagent Based on Top7}

\author{
CB Boschek, ${ }^{\text {(a) }}$ DO Apiyo, ${ }^{\text {(a) }}$ TA Soares, ${ }^{\text {(a) }}$ HE Engelmann, ${ }^{\text {(a) }}$ NB Pefaur, ${ }^{\text {(a) }}$ TP Straatsma, ${ }^{\text {(a) }}$ \\ and CL Baird ${ }^{(a)}$
}

(a) Pacific Northwest National Laboratory, Richland, Washington

Top $7_{C B 1}$, designed by EMSL users from the Pacific Northwest National Laboratory, is a synthetic protein that can specifically bind a protein targeted by the human immunodeficiency virus, or HIV, that can lead to AIDS. Top $7_{C B 1}$ can also be used as an inexpensive and effective alternative to antibodies. The work is the cover story in the May 2009 issue of Protein Engineering Design \& Selection (Figure 1).

Antibodies are one of the weapons used to fight disease or detect harmful substances. These proteins are one of the most commonly used reagents in the laboratory because they can be used to bind, recognize and quantify specific targets, such as toxins or proteins that specify different disease states.

Antibodies can be generated against virtually any target by immunization or by in vitro selection. However, they are large and frequently unstable, which makes them difficult to use for many practical applications. As a result, scientists are working to engineer smaller antibody fragments that remain specific and stable while being easy to produce. But again, designing these engineered fragments to be structurally robust while retaining their binding specificity has been difficult.

An emerging alternative is the use of intrinsically stable proteins as scaffolds for the generation of novel binding agents instead of generating natural or engineered antibodies. In fact, these novel scaffolds could be used in adverse environments such as those found in parts of the human body or in industry, where

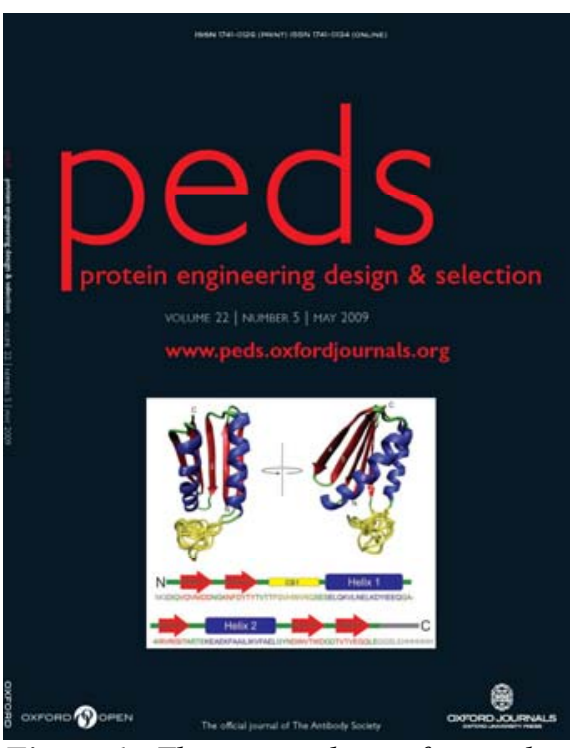

Figure 1. This research was featured on the cover of the May 2009 issue of Protein Engineering Design \& Selection. antibodies fail.

The aim of the study was to design a highly stable affinity reagent — a specific binding molecule — based on the synthetic protein Top 7 to assess its viability as a general affinity scaffold. Top7 is a small protein computationally designed by University of Washington scientists to be extremely stable. Its small size, known structure and very stable configuration make it an ideal scaffold for an affinity reagent.

The researchers selected a site in Top7 to insert CB1, a peptide constructed from a well-characterized peptide, PDP-CB1, that comes from a region of an anti-CD4 antibody. CD4 is a protein on the surface of immune cells that helps protect against infections such as HIV. Inserting this peptide resulted in the variant called Top $7_{\mathrm{CB} 1}$. Team members then evaluated the structural effect of the variant using molecular 
dynamics simulations that suggested that $\mathrm{Top}_{\mathrm{CB} 1}$ retains conformational stability at temperatures greater than $100^{\circ} \mathrm{C}$ - hotter than boiling water.

The modified Top7 also bound CD4 and, consistent with simulations, was extremely resistant to thermal and chemical structural change - retaining its secondary structure up to at least $95^{\circ} \mathrm{C}$. This CD4-specific protein demonstrates the functionality of Top7 as a viable scaffold for use as a general affinity reagent that could serve as a robust and inexpensive alternative to antibodies.

The PNNL team is currently using complementary determining region loops harvested from antibody libraries at PNNL as diversity elements for building libraries of Top7 variants for selection through yeast surface display. Based on suggestions that the destabilization effects of multiple loop addition are not synergistic, they are also exploring the use of multiple binding sequences at other regions within Top 7 to further increase specificity and affinity.

This work was supported by the U.S. Department of Energy with Laboratory Directed Research and Development funding through the Biomolecular Systems Initiative.

\title{
Citation
}

Boschek CB, DO Apiyo, TA Soares, HE Engelmann, N Pefaur, TP Straatsma, and CL Baird. 2009. "Engineering an ultra-stable affinity reagent based on Top7." Protein Engineering, Design \& Selection 22(5):1-8, doi:10.1093/protein/gzp007.

\section{Identification of a Putative Protein Profile Associating with Tamoxifen Therapy Resistance in Breast Cancer}

\author{
A Umar, ${ }^{(a)}$ H Kang,,$^{(b)}$ AM Timmermans, ${ }^{(a)}$ MP Look, ${ }^{(a)}$ ME Meijer-van Gelder, ${ }^{(a)}$ MA den \\ Bakker, ${ }^{(a)}$ N Jaitly, ${ }^{(b)}$ JWM Martens, ${ }^{(a)}$ TM Luider, $^{\left({ }^{(a)}\right.}{ }^{\text {JA Foekens, }},{ }^{(a)}$ and L Pasa-Tolic ${ }^{(b)}$ \\ (a) Erasmus Medical Center, Rotterdam, The Netherlands \\ (b) EMSL, Richland, Washington
}

The drug tamoxifen is used to treat breast cancer; however only about 50 in 100 people taking tamoxifen respond. Of those that respond, the majority eventually develop a resistance to the drug. This study provides data that could aid in isolating a protein or set of proteins that would predict if a patient will respond to the drug or if a different treatment is needed.

At EMSL, researchers from Erasmus Medical Center Rotterdam and EMSL studied cancerous cells and identified 55 proteins that vary in abundance between patients responsive to the breast cancer treatment tamoxifen and those that are not. The cells were microdissected from larger tumors. This exacting process, done by scientists at the Rotterdam center, results in pure samples of cancerous cells. The samples, constituting only about 3000 cells, were then packed by experts at EMSL into small innerdiameter capillaries and processed through EMSL's liquid chromatography/Fourier transform mass spectrometer. The data were analyzed using the accurate mass and tag approach. Using this process, the team identified 2000 proteins from each sample procured by laser microdissection.

Of these proteins, 55 were different in abundance between those that responded to tamoxifen and those that did not, indicating that a biomarker for resistance to this drug might exist (Figure 1). 
This study shows the effectiveness of using the accurate mass and tag approach with relatively small clinical samples. With this approach, 2000 proteins were identified that differed between tumors extracted from patients resistant to tamoxifen and those that were not. Previous studies that employed a small number of cells $(<10,000$ cells $)$ typically identified 20 times fewer proteins.

This research, funded by the National Institutes of Health's National Center for Research, was featured in Molecular \& Cellular Proteomics. MCP.

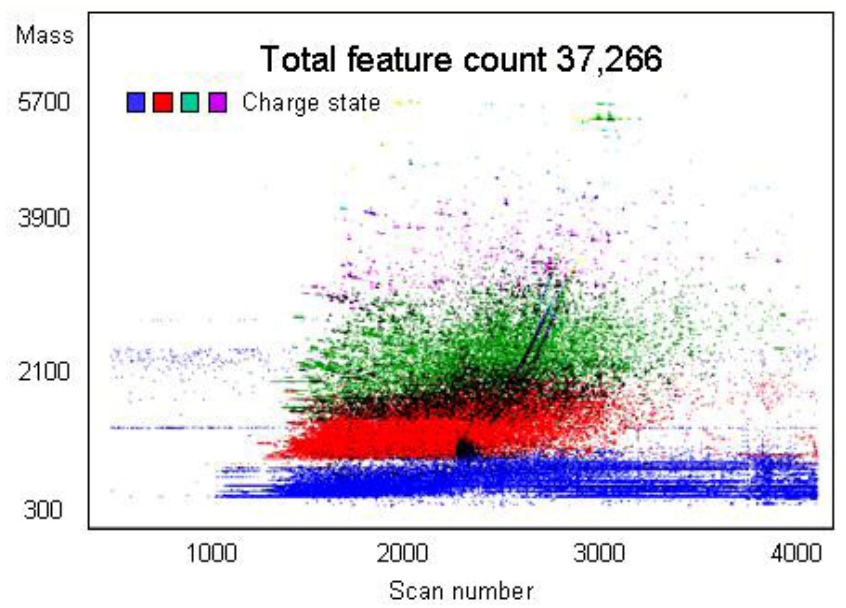

Figure 1. EMSL users determined the relative abundance of thousands of peptides, which led to the identification of 55 proteins that were different in abundance between the patients that responded to the drug and those that did not.

\title{
Citation
}

Umar AN, H Kang, AM Timmermans, MP Look, ME Meijer-van Gelder, MA den Bakker, N Jaitly, JW Martens, TM Luider, JA Foekens, and L Pasa-Tolic. 2009. "Identification of a Putative Protein Profile Associating with Tamoxifen Therapyresistance in Breast Cancer." Molecular \& Cellular Proteomics. MCP. doi:10.1074/mcp.M800493-MCP200

\section{Syndecan-1 Mediates the Coupling of Positively Charged Submicrometer Amorphous Silica Particles with Actin Filaments across the Alveolar Epithelial Cell Membrane}

\author{
G Orr, ${ }^{(a)}$ DJ Panther, ${ }^{\text {(a) }}$ KJ Cassens, ${ }^{\text {(a) }}$ JL Phillips, $^{\text {(a) }}$ BJ Tarasevich, ${ }^{\text {(a) }}$ and JG Pounds ${ }^{(a)}$ \\ (a) Pacific Northwest National Laboratory, Richland, Washington
}

By combining single-molecule microscopy and nanoparticle engineering at EMSL, scientists from Pacific Northwest National Laboratory showed how nano-sized particles interact with lung cells. Their results, which were published on the cover of Toxicology and Applied Pharmacology, provide insights about the mechanisms underlying of inhaled nanomaterials.

Dr. Galya Orr and her colleagues found that some positively charged nanomaterials seek out a specific cellular protein that gives them a free pass into alveolar type II epithelial cells in the lungs. The protein, known as syndican-1, is an integral plasma membrane protein that participates in cell proliferation, migration and organization.

Alveoli are tiny air sacs within the lungs where oxygen and carbon dioxide are exchanged. The alveolar type II cell regulates surfactant (wetting agent) metabolism, ion transport and alveolar repair in the lung. These cells are a potential target for inhaled engineered nanoparticles, which can cause inflammation and lead to respiratory diseases.

The researchers showed that positively charged nanomaterials attach to syndican- 1 on the plasma membrane of cells. This specific attachment is critical to internalizing nanoparticles in alveolar cells, 
which do not accumulate larger particles. The consequence of this interaction may be inflammation and, eventually, disease or migration of the nanoparticles into the bloodstream.

Sand-like synthetic amorphous silica particles at the submicron scale $\left(<10^{-6}\right.$ or 1 millionth of a meter) and nanoscale $\left(<10^{-9}\right.$ or 1 billionth of a meter) are being explored for drug delivery and medical imaging and sensing. These particles have been also used in a wide array of industrial applications, such as the food, cosmetic and paint industries, creating a significant source of potential human exposure through inhalation.

Much is already known about how microscale particles such as bacteria or combustion byproducts get into and affect cells. And, in fact, the human body has evolved cells and molecular processes to deal with these larger particles.

According to Orr, "Knowing more about the cellular interactions and fate of the particles, which drive the cellular response, and ultimately determine the impact on human heath, will help us to understand what makes a particle toxic or biocompatible."

The scientists used time-lapse fluorescence imaging at EMSL

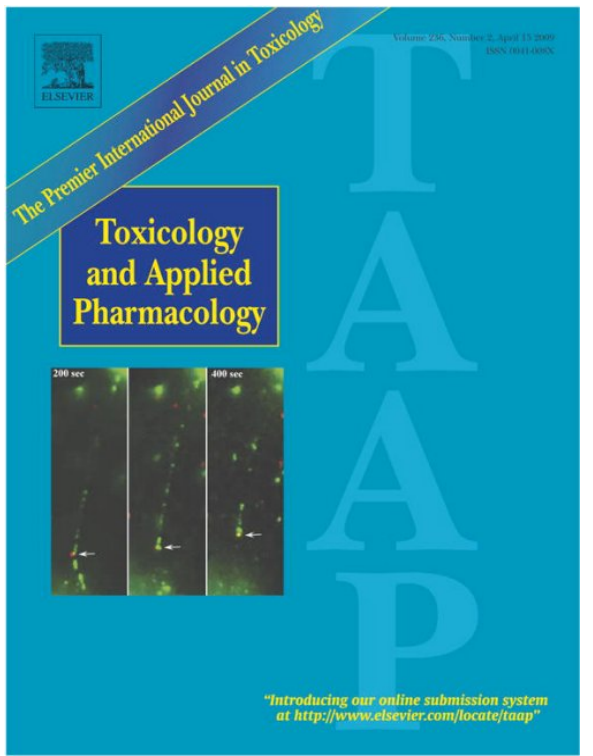

Figure 1. This research was featured on the cover of the April 15, 2009, issue of Toxicology and Applied Pharmacology. and materials science capabilities at PNNL to follow one particle at a time as it interacts with the living cell. This approach allowed them to identify cellular processes and molecular interactions that could not be otherwise observed.

Because nanomaterials come in many sizes, shapes, and physical properties, more research is needed to understand how cells process materials with other properties, and the intracellular fate. Because it is impossible to study each and every particle, the PNNL team is working to develop prediction approaches for nanotoxicity.

This work was supported by an Environmental Protection Agency STAR grant and the Air Force Research Laboratory through the Oregon Nanoscience and Microtechnologies Institute-Safer Nanomaterials and Nanomanufacturing Initiative and the Environmental Biomarkers Initiative at PNNL.

\section{Citation}

Orr G, DJ Panther, KJ Cassens, JL Phillips, BJ Tarasevich, and JG Pounds. 2009. "'Syndecan-1 Mediates the Coupling of Positively Charged Submicrometer Amorphous Silica Particles with Actin Filaments across the Alveolar Epithelial Cell Membrane." Toxicology and Applied Pharmacology 236(2):210-220. 


\title{
Calmodulin Mediates DNA Repair Pathways Involving H2AX in Response to Low-Dose Radiation Exposure of RAW 264.7 Macrophages
}

\author{
HS Smallwood, ${ }^{(a)}$ D Lopez-Ferrer, ${ }^{(b)}$ PE Eberlein, ${ }^{(a)}$ DJ Watson, ${ }^{(a)}$ and TC Squier ${ }^{(b)}$ \\ (a) Washington State University-Tri-Cities, Richland, Washington \\ (b) Pacific Northwest National Laboratory, Richland, Washington
}

Students taught by Dr. Heather Smallwood at Washington State University working side-by-side with scientists at Pacific Northwest National Laboratory identified a new pathway that cells take when exposed to low levels of radiation, such as those used to shrink tumors. The newly identified cellular pathway upregulates, or increases the cellular components of, DNA repair pathways in response to lowdose radiological exposures. Cell survival is enhanced through pathways involving phosphorylated histone $H 2 A X$ - the addition of phosphorous to the chief protein components of the complex combination of DNA, RNA, and protein that makes up chromosomes. These DNA repair pathways are distinct from other protein complex pathways tested that enhance cell death in an attempt to remove damaged cells in response to radiation. Such removal systems prevent the damaged cells from sapping further nutrients from an organism and act to halt further spread of infection.

As part of the mammalian immune system, macrophages, or white blood cells, normally recognize and remove dead cells and pathogens while further stimulating an immune response. This capability makes them a first line of defense against disease. Identifying a dose-dependent increase in the expression level of the calcium signaling protein, calmodulin $(\mathrm{CaM})$, in irradiated mouse macrophage cells indicates that such increases are part of a specific radiation-dependent cellular response that can help expedite DNA damage repair (Figure 1).

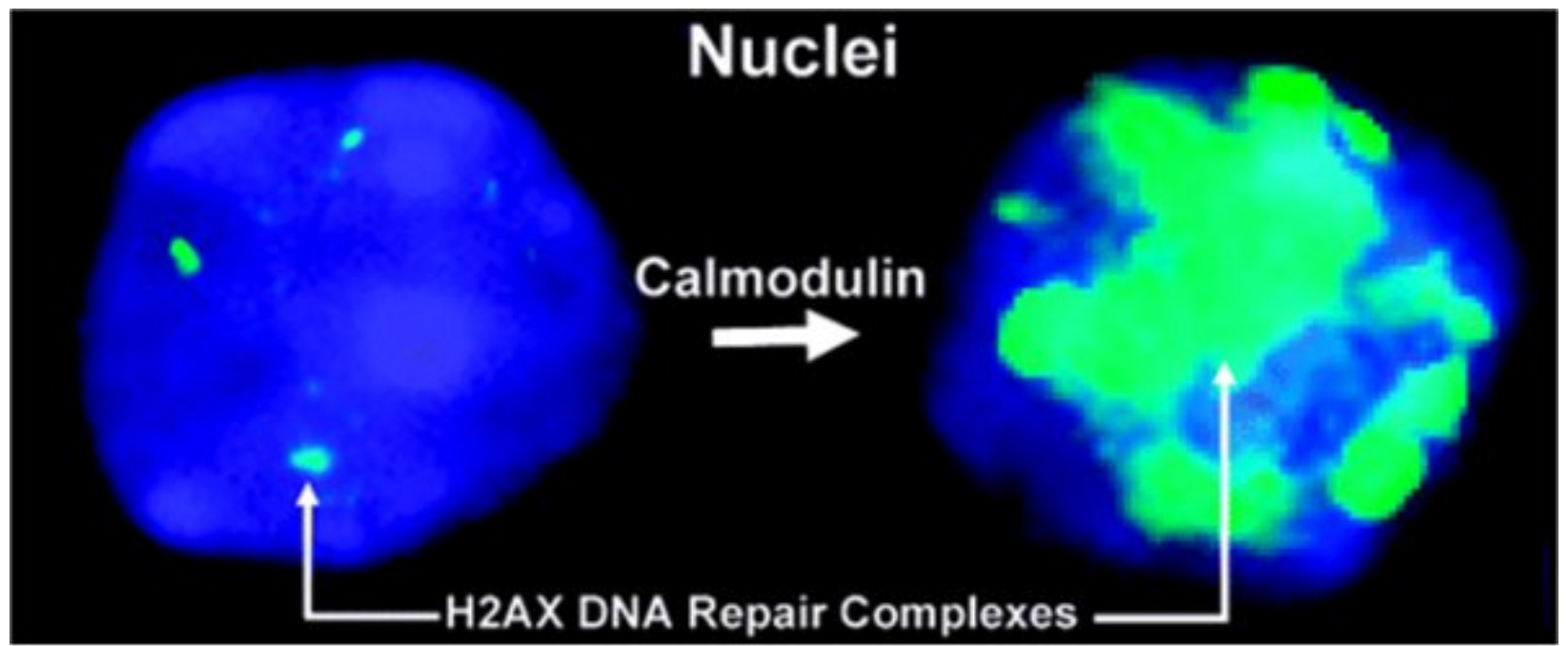

Figure 1. Visualization of DNA repair complexes (green) within DAPI-stained nucleus (blue) of macrophage cell, demonstrating augmentation of repair pathways associated with the upregulation of the regulatory protein calmodulin.

Understanding the molecular mechanisms that modulate macrophage resistance to radiation is necessary for developing effective radiation therapies, because tumor-associated macrophages promote processes that enhance the spread of cancer. The increases in CaM abundance in response to the lower radiation doses used in the study suggest a possible role of $\mathrm{CaM}$ in mediating cellular response pathways to 
clinically relevant doses in radiation therapy. Because phosphorylated histone $\mathrm{H} 2 \mathrm{AX}$ acts as a universal organizing center that both anchors chromosomal ends and mediates DNA repair, these results are broadly significant for understanding the radioresistance mechanisms of macrophages and other bloodgenerating cell types in response to radiation exposure.

The results suggest that therapeutic treatments that target $\mathrm{CaM}$ in conjunction with traditional radiotherapies may help kill tumor-associated macrophages, thereby restricting cancer cell proliferation and tumor metastisis. Such treatments are likely to have other beneficial effects because CaM antagonists are known to prevent cancer invasiveness.

To better understand the molecular basis for the sensitivity of macrophages to low therapeutic doses of ionizing radiation, the research team investigated the possible role of CaM in modulating double-stranded DNA damage. CaM is suggested to be a principal mechanism of radiation-induced cellular death and transformation. They grew and irradiated mouse macrophage leukemia cells (i.e., RAW 264.7), then assayed cell survival to identify cells undergoing programmed death, or apoptosis, and to assess DNA damage and repair pathways, changes in the expression level of $\mathrm{CaM}$, and total protein content.

After macrophage irradiation, increases in $\mathrm{CaM}$ abundance resulted in an increase in the number of phosphorylated histone H2AX foci, associated with DNA repair, with no change in the extent of doublestranded DNA damage. Measurement of the radiation sensitivity of RAW 264.7 macrophages, using a well-established survival assay for studying the effectiveness of specific agents on the survival and proliferation of cells, showed that macrophages are more resistant to high radiation doses than low. Altering CaM levels and protein complex NFkB-dependent pathways was found to have multiple effects, some of which may disrupt DNA damage response pathways and cellular apoptosis. CaM overexpression reduced radiation-dependent cell killing and disrupted the adaptive cellular response to low-dose radiation, while radiation-induced DNA damage was shown to be insensitive to CaM. Upregulation of CaM abundance enhanced DNA repair pathways after irradiation. Hence, Laboratory Fellow Thomas Squier emphasized that "the medical significance of the finding is that specifically inhibiting repair mechanisms in tumor cells and tumor associated macrophages before radiation damage occurs will enable more rapid and targeted killing of these cells upon treatment, which is the main challenge in successful radiation therapies."

Future measurements will need to identify the components of the CaM-dependent pathway that leads to histone H2AX phosphorylation, thereby activating DNA repair and enhancing macrophage survival following radiation exposure.

This work was completed as part of the student radiobiology laboratory associated with Washington State University Tri-Cites, Richland. A portion of the research was performed using an LTQ-Orbitrap mass spectrometer and a custom-built liquid chromatography system at EMSL. Results were published in Chemical Research in Toxicology.

\section{Citation}

Smallwood HS, D Lopez-Ferrer, PE Eberlein, DJ Watson, and TC Squier. 2009. "Calmodulin Mediates DNA Repair Pathways Involving H2AX in Response to Low-Dose Radiation Exposure of RAW 264.7 Macrophages." Chemical Research in Toxicology 22(3):460-470. doi:DOI: 10.1021/tx800236r 


\title{
Proteomic Investigation of the Time Responses of RAW 264.7 Macrophages to Infection with Salmonella enterica
}

\author{
L Shi, ${ }^{(a)}$ SM Chowdhury, ${ }^{(a)} H S$ Smallwood, ${ }^{(a)}$ H Yoon, ${ }^{(b)} H M$ Mottaz-Brewer, ${ }^{(c)}$ AD Norbeck, ${ }^{(a)}$ \\ JE McDermott, ${ }^{\text {(a) }}$ TRW Clauss, ${ }^{(a)}$ F Heffron, ${ }^{\text {(b) }}$ RD Smith, $^{(a)}$ and JN Adkins ${ }^{(a)}$ \\ (a) Pacific Northwest National Laboratory, Richland, Washington \\ (b) Oregon Health Sciences University, Portland, Oregon \\ (c) EMSL, Richland, Washington
}

EMSL researchers and users from Pacific Northwest National Laboratory and Oregon Health Sciences University applied a novel global proteomic approach to better understand the extent to which macrophages respond to infection by a form of Salmonella. Recognized as the first global proteomic analyses of time course responses of mouse macrophages to S. enterica serotype Typhimurium (S. Typhimurium) infection, the study may lead to new strategies for diagnosing, treating, and vaccinating against infectious disease.

Macrophages, from the Greek for "eating cells," are white blood cells that act as cellular scavengers by ingesting dying and invading bacterial cells, but they recognize and refuse to eat their own kind. The macrophages swallow the bacterial cells and then release powerful anti-bacterials that prevent bacteria from further dividing and infecting host cells. S. Typhimurium, which can be transmitted by ingesting contaminated food or water, is a pathogen that grows in the gastrointestinal tract of many animal species.

By infecting susceptible mouse macrophages with S. Typhimurium and using global proteomics to analyze the proteins at various time points following infection, researchers identified 1,006 macrophage proteins (Figure 1). The peptide abundances of 244 macrophage proteins, or 24 percent of the total macrophages identified, changed significantly after infection. The functions of the Salmonella-affected macrophage proteins were diverse, including production of antibacterial nitric oxide, production of prostaglandin $\mathrm{H}_{2}$, and regulation of intracellular traffic. The diversity in functions demonstrated a global macrophage response to Salmonella infection.

The researchers used Western Blot analysis to confirm the proteomic results. The analysis also

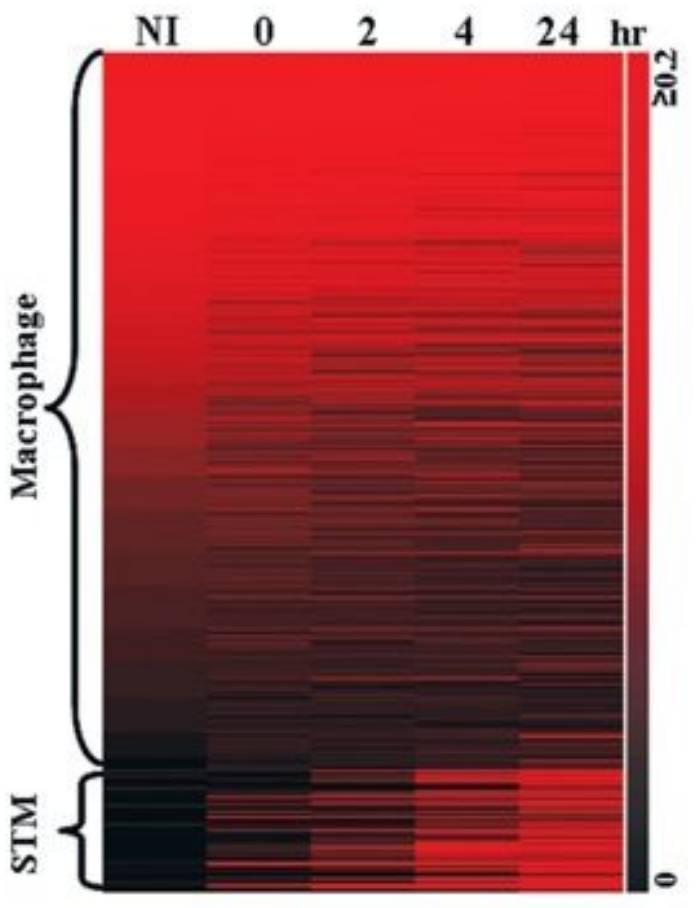

Figure 1. Peptide abundances of all identified macrophage and S. Typhimurium (STM) proteins. A total of 1121 proteins are shown, which include 1006 macrophage and 115 STM proteins. Peptide abundances for all identified macrophage and STM proteins in the non-infection (NI) control and at different time points of infection are indicated by colors that range from black (peptide abundance $=$ $0 \%$ ) to red (peptide abundance $\geq 0.2 \%$ ). revealed that Salmonella infection increases mitochondrial abundance of the enzyme superoxide dismutase and decreases the abundance of the sorting nexin protein SNX6, most likely through the 
bacterial virulence factor SopB. Superoxide dismutase acts like a natural antioxidant, repairing cells and reducing the damage caused by superoxide. Sorting nexin proteins help cells move proteins to the correct location within the endomembrane system.

Macrophages are critical in autoimmune diseases such as rheumatoid arthritis, diabetes, and multiple sclerosis. They also play a role in HIV infection and can stimulate cancer cells. Researchers gain valuable information about the strengths and weaknesses of our immune system using mouse cell models such as used here. This study leads to a better understanding of macrophage responses to infection, provides insight into disease development, and suggests targets for therapeutic intervention.

Salmonella-infected macrophages are regularly used to show the molecular mechanisms essential for the interaction between macrophage and intracellular pathogens. Macrophages are important in controlling Salmonella-mediated systemic infection in susceptible mice. They also help control morbidity and mortality in unvaccinated infected mice, while helping eliminate $\mathrm{S}$. Typhimurium in vaccinated mice.

Using a liquid chromatography-mass spectrometry proteomic approach, the researchers analyzed the time course responses of the cell lysate of mouse RAW 264.7 macrophages after infection with $S$. Typhimurium. They studied samples collected at four different time points following infection $(0,2,4$, and 24 hours). Future work will focus on understanding the roles of Salmonella-affected macrophage proteins that could lead to improved host-based therapeutics to intracellular pathogens.

This work was supported by the Laboratory Directed Research and Development program at PNNL and the National Institute of Allergy and Infectious. The research was featured in Infection and Immunity.

\section{Citation:}

Shi L, SM Chowdhury, HS Smallwood, H Yoon, HM Mottaz-Brewer, AD Norbeck, JE McDermott, TRW Clauss, F Heffron, RD Smith, and JN Adkins. 2009. "Proteomic Investigation of the Time Responses of RAW 264.7 Macrophages to Infection with Salmonella enterica." Infection and Immunity 77(8):3227-3233 


\section{Geochemistry/Biogeochemistry and Subsurface Science}

One of the most challenging issues confronting DOE is the safe and cost-effective management of environmental pollutants and the remediation of hazardous waste sites. DOE is responsible for managing some 40 million cubic meters of contaminated soils and 1.7 trillion gallons of contaminated groundwater. At the Hanford Site in Richland, Washington, alone, DOE invests $\sim \$ 2$ billion a year in cleanup activities related to storing and processing radioactive waste in underground tanks and extensive remediation of soils and groundwater contaminated by past disposal practices and tank waste leaks. These issues are also national problems. For example, across the United States, thousands of Superfund sites exist with various levels and types of contamination such as organics, heavy metals, inorganics, and radionuclides. In the future, the subsurface migration of the byproducts of energy production, including nuclear waste and carbon dioxide, represents one of the most daunting challenges to DOE, and the safe and cost-effective disposal of these byproducts could be a limiting factor in deploying new energy technologies for the nation.

Molecular-level processes, such as aqueous complexation, adsorption to different mineral phases, or microbial reduction of redox active metals, often control the transport and fate of contaminants in the environment. These processes occur in chemically and physically heterogeneous subsurface environments. Understanding the structure, chemistry, and nanoscale geometric properties of mineral/water and microbe/mineral interfaces are critical to a mechanistic understanding of subsurface reactivity and contaminant transport. As a result, molecular-level studies of interfacial geochemistry and biogeochemical reactions have been an active area of research for more than a decade. Unraveling these phenomena at the molecular level and determining their impact on contaminant migration and transformation is a key objective of this science theme.

This science theme will focus EMSL's scientific resources on the following key topical areas:

- Fundamental studies of interfacial molecular geochemistry and biogeochemistry

- Unraveling the chemistry of radionuclides in the subsurface

- Developing predictive transport models of chemical and microbial species in the subsurface.

Research in the area of biogeochemistry and subsurface science is well established in EMSL. EMSL proposes to build on its strength in this area by advancing our capabilities to determine the chemical form of contaminants in complex subsurface materials, develop a fundamental understanding of dynamic interfacial processes and their impact on observed reactivity, and to improve our linkage of fundamental studies of molecular geochemistry/biogeochemistry to field scale transport processes.

Highlights of research performed in Fiscal Year 2008 under EMSL's Geochemistry/Biogeochemistry and Subsurface Science theme are shown below. 


\title{
Enhanced Remedial Amendment Delivery through Fluid Viscosity Modifications: Experiments and Numerical Simulations
}

\author{
L Zhong, $^{(a)}$ M Oostrom, ${ }^{(a)}$ TW Wietsma, ${ }^{(b)}$ and MA Covert ${ }^{(a)}$ \\ (a) Pacific Northwest National Laboratory, Richland, Washington \\ (b) EMSL, Richland, Washington
}

Remediation efforts are often incomplete and laborious because of subsurface contaminants located in hard-to-reach places, such as areas of low permeability in aquifer systems. Using resources at EMSL, a Pacific Northwest National Laboratory and EMSL research team found a way to enhance cleanup effectiveness and efficiency by incorporating the inexpensive and readily available polymer, Xanthan gum, into the remediation process. Advances in remediation research may lead to improved remediation techniques, positively affecting the environment and human health.

Using flow cell experiments, the Pacific Northwest National Laboratory and EMSL research team simulated the remediation of contaminated areas. The team found that adding Xanthan gum to remediating solutions increased their viscosity, thus helping deliver the remediating agent to areas in which the contaminant may otherwise have been left behind and increasing the portion of the contaminated volume touched by the remediating agent (Figure 1). Further, a version of the Subsurface Transport over Multiple Phases simulator - a general-purpose tool developed by PNNL scientists for simulating subsurface flow and transport -that was modified to account for using the polymer in the system accurately predicted the results of the experiments. The modified version of STOMP may be used to predict subsurface
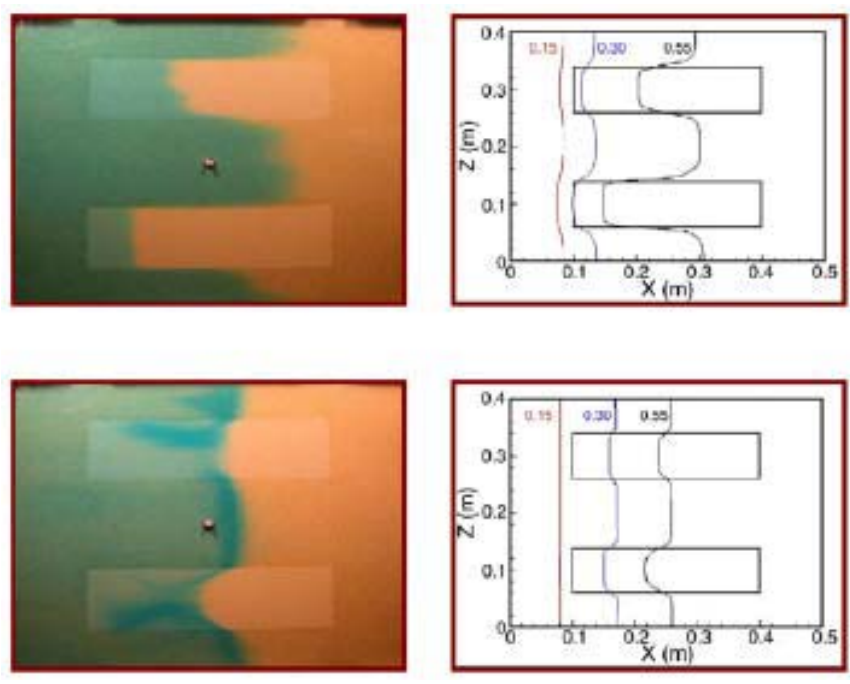

Figure 1. Xanthan gum (lower left) helps remediating agents reach areas of low permeability as compared to a control (upper left) in flow cell experiments (permeated volume shown in blue). STOMP predicts the experimental results well (right). remediation performance in similar systems at larger scales, and the Xanthan gum additive may prove useful in real-world scenarios such as cleanup of uranium-contaminated areas.

Such advances in remediation research demonstrate the feasibility of an inexpensive alternative remediation technique and of using computational tools to predict the effectiveness of such techniques under real-world conditions. In addition, this research supports EMSL's goal to link theory with experiment.

The team's work has led to a Department of Defense Environmental Security Technology Certification Program project, Enhanced Amendment Delivery to Low Permeability Zones of Chlorinated Solvent Source Area Bioremediation. For this project, PNNL researchers are collaborating with colleagues from GSI Environmental. 
The research, supported by PNNL's Laboratory Directed Research and Development program, was published in Journal of Contaminant Hydrology.

\title{
Citation
}

Zhong L, M Oostrom, TW Wietsma, and MA Covert. 2008. "Enhanced remedial amendment delivery through fluid viscosity modifications: Experiments and numerical simulations" Journal of Contaminant Hydrology 101:29-41.

\section{Kinetics of Reduction of Fe(III) Complexes by Outer Membrane Cytochromes MtrC and OmcA of Shewanella oneidensis MR-1}

\author{
Z Wang, ${ }^{(a)}$ L Shi, ${ }^{(a)} C$ Liu, ${ }^{(a)} X$ Wang, ${ }^{(a)} M J$ Marshall, ${ }^{(a)} J M$ Zachara, ${ }^{(a)} K M$ Rosso, ${ }^{(a)} M$ Dupuis, ${ }^{(a)}$ \\ JK Fredrickson, ${ }^{(a)}$ and SM Heald ${ }^{(b)}$ \\ (a) Pacific Northwest National Laboratory, Richland, Washington \\ (b) Argonne National Laboratory, Argonne, Illinois
}

New details about how bacteria and metals interact highlight the importance of considering metal-ligand complexes as part of bioremediation strategies. Bacteria such as Shewanella oneidensis MR-1 hold promise as a bioremediation tool because they exchange electrons with metals, affecting their solubility and thus their level of danger to the environment and human health. This work may lead to enhanced bioremediation strategies to remedy contaminated environments, such as the DOE's Hanford Site in Richland, Washington.

As part of EMSL's Biochemistry Grand Challenge, scientists have made significant progress toward understanding electron exchange between bacteria and metals. A research team led by EMSL users from the Pacific Northwest National Laboratory carried this Grand Challenge further by using spectroscopy and computational tools at EMSL to determine the kinetics of electron exchange when the metal, iron, is coupled to ligands of geological and environmental significance.

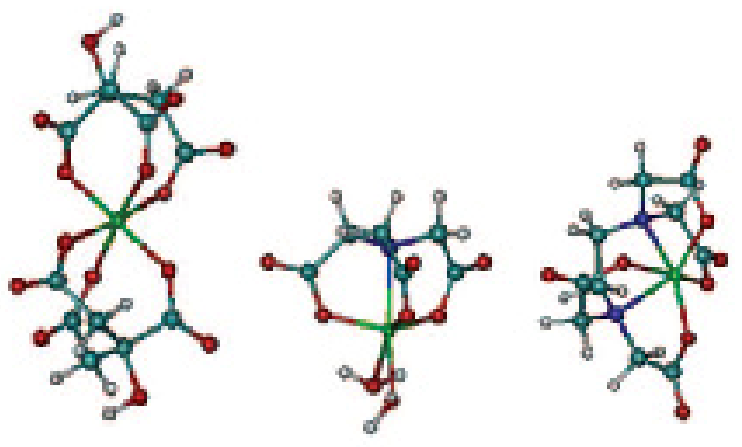

Figure 1.Computed structures of $\mathrm{Fe}$-(citrate) ${ }_{2}^{3-}$ (left), $\mathrm{FeOH}$ NTA' (middle), and Fe-EDTA' (right).

In particular, the research team determined how Fe(III) complexes with the ligands citrate, nitrilotriacetic acid (NTA), and ethylenediaminetetraacetic acid (EDTA) were reduced by two Shewanella surface proteins known to be involved in electron transfer: MtrC and OmcA (Figure 1). The team's results were surprising: even though electron transfer from the surface proteins to the Fe(III) EDTA complex is thermodynamically unfavorable compared to reactions involving $\mathrm{Fe}(\mathrm{III})$-citrate and $\mathrm{Fe}(\mathrm{III})-\mathrm{NTA}$, it happened most quickly. For the EDTA reactions, the reaction rate was influenced by the relatively large reorganization energies of the reactants - so much so as to override the strong thermodynamics. This and the geometry of the molecules in the reactions favored the electron transfer kinetics involving EDTA. The team's work demonstrates the importance of metal complexation to bioremediation. For 
contaminated sediments where radioactive metals are co-disposed with organic chelating agents, any effective bioremediation strategy should take into consideration the ligand complexation effect.

Experimental and computational studies such as these refine the understanding of the fundamental biological process of bacterial electron transfer and contribute to EMSL's goal to rapidly link theory and experiment. Further, such studies contribute heavily to EMSL's Biochemistry Grand Challenge, which supported this research.

This research was published in Applied and Environmental Microbiology.

\title{
Citation
}

Wang Z, L Shi, C Liu, X Wang, MJ Marshall, JM Zachara, KM Rosso, M Dupuis, JK Fredrickson, and SM Heald. 2008. "Kinetics of Reduction of Fe(III) Complexes by Outer Membrane Cytochromes MtrC and OmcA of Shewanella oneidensis MR-1." Applied and Environmental Microbiology 74(21):67466755.

\section{Antibody Recognition Force Microscopy Shows that Outer Membrane Cytochromes OmcA and MtrC are Expressed on the Exterior Surface of Shewanella oneidensis MR-1}

\author{
BH Lower, ${ }^{(a)} R$ Yongsunthon, ${ }^{(b)}$ L Shi, ${ }^{(c)}$ L Wildling, ${ }^{(d)} H G$ Gruber, ${ }^{(d)} N S$ Wigginton, ${ }^{(e)}$ \\ CL Reardon, ${ }^{(c)}$ GE Pinchuk, ${ }^{(c)}$ TC Droubay, ${ }^{(c)}$ JF Boiley, ${ }^{(f)}$ and SK Lower ${ }^{(a)}$ \\ (a) The Ohio State University, Columbus, Ohio \\ (b) Corning, Inc., Corning, New York \\ (c) Pacific Northwest National Laboratory, Richland, Washington \\ (d) Johannes Kepler University of Linz, Linz, Austria \\ (e) Ecole Polytechnique Federale de Lausanne, Lausanne, Switzerland \\ (f) Umea University, Umea, Sweden
}

Electron exchange is a basic function carried out by bacteria such as Shewanella. Understanding the mechanism of this exchange is of fundamental importance and may lead to advanced bioremediation strategies.

An international team used EMSL surface science and imaging capabilities to determine the location, with nanoscale resolution, of MtrC and OmcA - two Shewanella oneidensis MR-1 surface proteins that can affect environmental quality. Bacteria such as Shewanella exchange electrons with minerals, yielding effects such as changes in the migration of environmental contaminants and water purity. MtrC and OmcA are cytochromes, or surface-bound iron-containing proteins that facilitate this exchange between Shewanella and iron. Carried out, in part, as a contribution to EMSL's Biogeochemistry Grand Challenge, the team's imaging studies offer a deeper understanding of the role MtrC and OmcA play in electron exchange and may lead to enhanced bioremediation methods.

Comprised of participants from The Ohio State University; Pacific Northwest National Laboratory; Corning Incorporated, Johannes Kepler University of Linz, Austria; Ecole Polytechnique Fédérale de Lausanne, Switzerland; and Umeå University, Sweden, the research team used EMSL's oxygen plasmaassisted molecular beam epitaxy capability to grow hematite $\left(\mathrm{Fe}_{2} \mathrm{O}_{3}\right)$ thin films. Shewanella cells were allowed to attach to the thin films; the iron in the hematite serves as an electron acceptor. EMSL's 
dynamic force-scanning probe microscope for single-molecule force spectroscopy as well as antibody-recognition force microscopy (Ig-RFM) were then used to map the locations of $\mathrm{MtrC}$ and OmcA on the live Shewanella surface (Figure 1). A relatively new technique, IgRFM uses a nanometer-scale, flexible antibody-coated tip that is moved across a sample surface. When the antibody, in
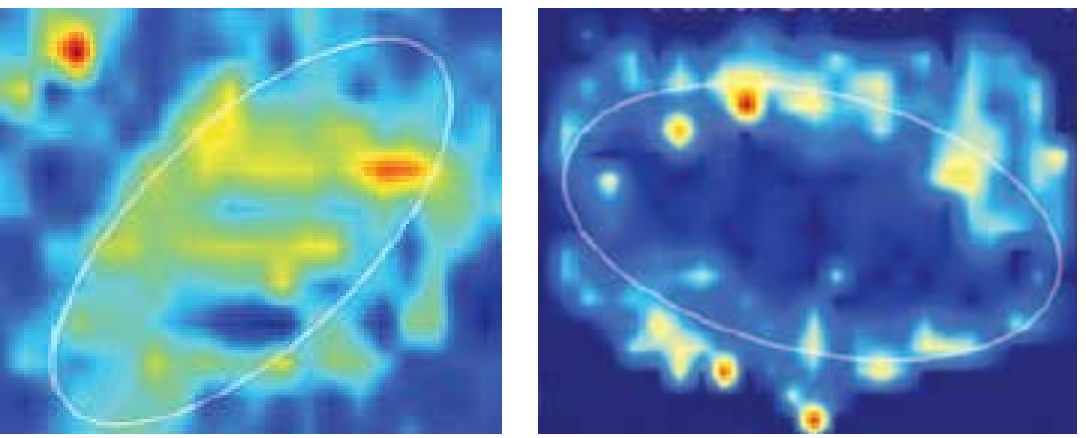

Figure 1. Ig-RFM showed that MtrC is distributed across the Shewanella surface (left). OmcA is localized to the cell-hematite interface (right). Both $M \operatorname{tr} C$ and $O m c A$ are in the EPS (white lines indicate the bacterium boundary).

this case anti-MtrC or anti-OmcA, comes in contact with its binding partner, a measurable force is required to separate the two. Force measurements indicate that MtrC is distributed rather uniformly on the bacterial surface, and OmcA is localized at the cell-mineral interface. Both cytochromes locate to the extracellular polymeric substance, which is made up of the secretions that help bacteria bind to surfaces.

Understanding microbe-mineral electron exchange is important to developing enhanced bioremediation methods for contaminated environments, such as the DOE's Hanford Site in Richland, Washington.

This work was supported by DOE's Office of Basic Energy Sciences' Geosciences Research Program, EMSL's Biogeochemistry Grand Challenge, DOE Office of Biological and Environmental Research's Genomics-Genomes to Life Program, and the National Science Foundation. It was featured on the cover of the May 1, 2009, issue of Applied and Environmental Microbiology.

\title{
Citation
}

Lower BH, R Yongsunthon, L Shi, L Wildling, HJ Gruber, NS Wigginton, CL Reardon, GE Pinchuk, TC Droubay, JF Boily, and SK Lower. 2009. "Antibody Recognition Force Microscopy Shows that Outer Membrane Cytochromes OmcA and MtrC Are Expressed on the Exterior Surface of Shewanella oneidensis MR-1." Applied and Environmental Microbiology 75(9)2931-2935.

DOI:10.1128/AEM.02108-08.

\section{Surface and Interfacial Properties of Nonaqueous-Phase Liquid Mixtures Released to the Subsurface at the Hanford Site}

\author{
SR Nellis, ${ }^{\left({ }^{a}\right)} \mathrm{H}$ Yoon, ${ }^{(a)}$ CJ Werth, ${ }^{(a)}$ M Oostrom, ${ }^{(b)}$ and AJ Valocchi ${ }^{\left({ }^{(a)}\right.}$ \\ (a) University of Illinois, Urbana, Illinois \\ (b) Pacific Northwest National Laboratory, Richland, Washington
}

Models developed by EMSL users that incorporate data from real-world scenarios will predict the fate and transport of environmental contaminants more accurately, enabling the development of better remediation techniques and the protection of human health and the environment. 
New results afforded by use of EMSL resources demonstrate the importance of accounting for complex organic liquid mixtures in flow and transport models. Basing its experiments on a real-world scenario, a research team led by Professor Charlie Werth from the University of Illinois and involving participants from the Pacific Northwest National Laboratory measured the surface and interfacial tensions of carbon tetrachloride $\left(\mathrm{CCl}_{4}\right)$ based nonaqueousphase liquids and a wastewater solution both composed of chemicals known to have been discharged at the DOE's Hanford Site in Richland, Washington (Figure 1). The surface and interfacial tensions of liquids contaminating the environment are of particular importance because they affect the ability of those solutions to migrate in the subsurface.

For their experiments, the team prepared six $\mathrm{CCl}_{4}$-based mixtures primarily containing

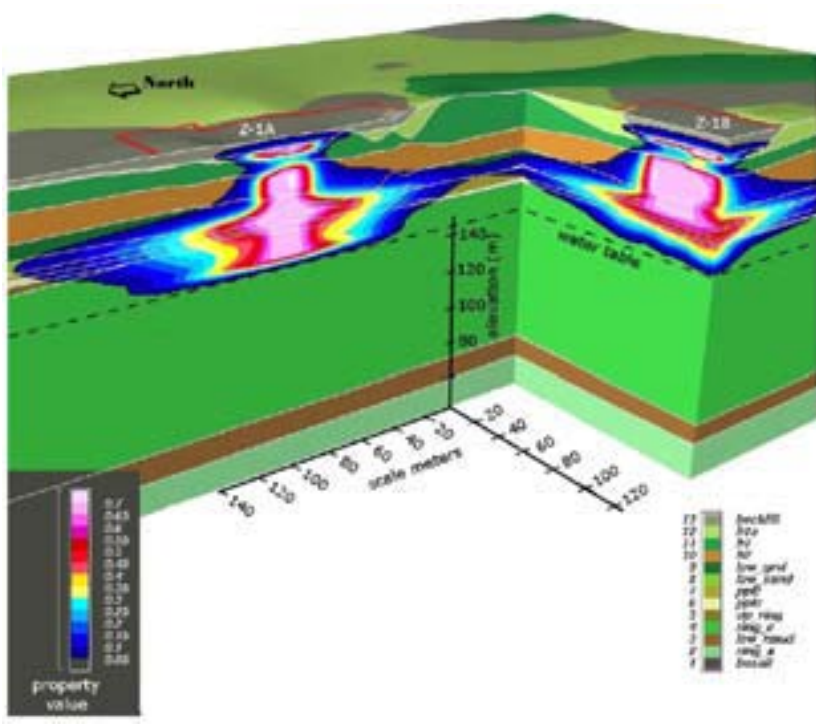

Figure 1. Modeling studies conducted by co-author Mart Oostrom to predict gaseous CCl4 concentrations for two Hanford Site disposal areas show that simulations using the Werth team's data (above, pink indicates highest CCl4 levels) yield larger contaminant plumes than simulations using single-component fluid properties. tributyl phosphate, dibutyl butyl phosphonate, and lard oil as well as a representative wastewater solution containing nitrates and metals. In comparison to pure $\mathrm{CCl}_{4}$, the $\mathrm{CCl}_{4}$-based mixtures had a significantly lower interfacial tension upon interacting with wastewater; the surface tensions of the $\mathrm{CCl}_{4}$-based mixtures compared to pure $\mathrm{CCl}_{4}$ were minimally affected. The chemicals added to $\mathrm{CCl}_{4}$ in solution also increased the mixtures' spreading coefficients such that if the mixtures were released to an uncontaminated area, they would spread quickly and contaminate larger areas of the subsurface than would pure $\mathrm{CCl}_{4}$. Adding another complication, the mixtures change over time; for example, $\mathrm{CCl}_{4}$ will volatize more quickly than the other components, leaving those components more concentrated in the mixture, thus lowering the mixture's interfacial tension. Per these results, the properties and behavior of chemical mixtures need to be accounted for in flow and transport models to provide accurate predictions and be most effectively used for remediation studies.

Real-world-based experiments such as these will lead to more enhanced fate and transport models for use by the remediation sciences and geochemistry communities. This work, primarily supported by DOE's Office of Biological \& Environmental Research's Environmental Remediation Sciences Program, was featured in Vadose Zone Journal.

\section{Citation}

Nellis SR, H Yoon, CJ Werth, M Oostrom, and AJ Valocchi. 2009. "Surface and Interfacial Properties of Nonaqueous-Phase Liquid Mixtures Released to the Subsurface at the Hanford Site" Vadose Zone Journal 8(2):343-351. 


\title{
Electron Donor-Dependent Radionuclide Reduction and Nanoparticle Formation by Anaeromyxobacter dehalogenans Strain 2CP-C
}

\author{
MJ Marshall, ${ }^{(a)}$ AC Dohnalkova, ${ }^{(b)}$ DW Kennedy, ${ }^{(a)}$ AE Plymale, ${ }^{(a)}$ SH Thomas, ${ }^{(c)}$ \\ FE Loffler, ${ }^{(c)}$ RA Sanford, ${ }^{(d)}$ JM Zachara, ${ }^{(a)}{ }^{\text {JK Fredrickson, }},{ }^{(a)}$ and AS Beliaev ${ }^{(a)}$ \\ (a) Pacific Northwest National Laboratory, Richland, Washington \\ (b) EMSL, Richland, Washington \\ (c) Georgia Institute of Technology, Atlanta, Georgia \\ (d) University of Illinois, Urbana, Illinois
}

Recent scientific findings show that the bacterium Anaeromyxobacter dehalogenans can efficiently use either organic carbon or hydrogen as an electron donor-or energy source - to reduce the solubility of uranium. This could benefit cleanup efforts at the U.S. Department of Energy's Hanford Site, where subsurface sediments have electron donor variability that may work with versatile bacteria such as Anaeromyxobacter to make uranium less risky to the environment.

A research team led by EMSL users from Pacific Northwest National Laboratory investigated mechanisms involved in reducing radionuclide contaminants by $A$. dehalogenans. At the Hanford Site, uranium and technetium are contaminants whose migrations in the subsurface are anticipated being a major risk factor to the Columbia River in the future. Biotransformation of uranium and technetium can reduce their mobility in the subsurface by converting them to less soluble forms, thus decreasing their eventual threat to the river.

Previously, scientists showed that $A$. dehalogenans coupled cellular growth with uranium reduction; however, they knew little about the biotransformation product of this reaction or the interaction of this bacterium with other radionuclides. In this study, scientists

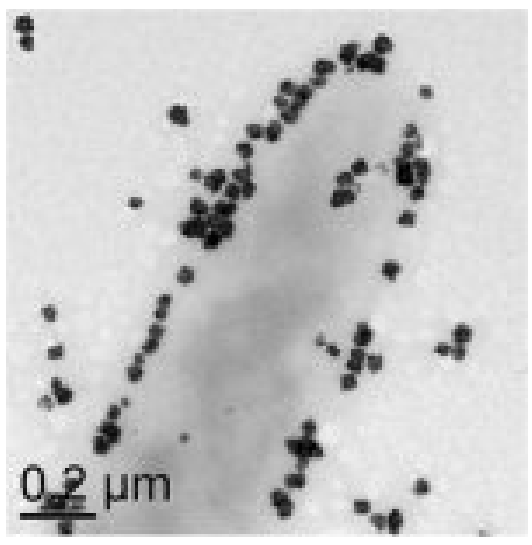

Figure 1. Transmission electron micrograph image of $A$. dehalogenans $2 C P-C$. observed that the electron donor source provided for uranium reduction influenced the reduction rates and the extent of aggregation of extracellular uraninite, a solid form of uranium resulting from microbial reduction. They saw that when Anaeromyxobacter cells were provided with an organic electron donor source, distinct nanoparticle aggregates formed that were ten times larger in diameter than individual nanoparticles produced during hydrogen oxidation. Similarly, individual extracellular technetium dioxide nanoparticles aggregated in clusters greater than $50 \mathrm{~nm}$ in diameter, although this was not linked to the electron donor source.

Using high-resolution electron microscopy (Figure 1), the scientists determined the localization, size and degree of aggregation of the nascent nanoparticles. These findings provide a better understanding of the importance of electron donor type on radionuclide reduction by Anaeromyxobacter and the nature of the insoluble oxide precipitates. They then used kinetic studies and transmission electron microscopy to determine the localization, size and degree of aggregation of the less-soluble technetium and uraninite nanoparticles. 
The results of this work provide the scientific community important insights into Anaeromyxobacter electron transfer to contaminants found at DOE sites that will aid in finding remediation solutions. Recent findings indicate that members of the Anaeromyxobacter genus contribute to metal and radionuclide reduction in contaminated groundwater and sediments at DOE's Oak Ridge Field Research Center in Tennessee. They may also be present at other contaminated DOE sites, including Hanford.

The results produced in this study suggest that Anaeromyxobacter uses biomolecular mechanisms similar to other model metal-reducing bacteria such as Geobacter and Shewanella to transform radionuclide contaminants. Further investigations will be needed to consider the biomolecular mechanisms contributing to metal reduction by Anaeromyxobacter and the physiochemical properties of the nanoparticulate end products and their long-term behavior in porous subsurface such as that at the Hanford Site.

This work, supported by DOE's Environmental Remediation Sciences Program, was featured in Environmental Microbiology.

\title{
Citation
}

Marshall MJ, AC Dohnalkova, DW Kennedy, AE Plymale, SH Thomas, FE Löffler, RA Sanford, JM Zachara, JK Fredrickson, and AS Beliaev. 2009. "Electron donor-Dependent Radionuclide Reduction and Nanoparticle Formation by Anaeromyxobacter dehalogenans Strain 2CP-C." Environmental Microbiology 11:534-543 (doi:10.1111/j.1462-2920.2008.01795.x).

\section{Reduction of $\mathrm{Hg}(\mathrm{II})$ to $\mathrm{Hg}(0)$ by Magnetite}

\author{
HA Wiatrowski,,$^{(a)(b)} S$ Das, ${ }^{(a)(c)}$ R Kukkadapu, ${ }^{(d)}$ ES Ilton, ${ }^{(e)} T$ Barkay, ${ }^{(a)}$ and N Yee ${ }^{(a)}$ \\ (a) Rutgers University, New Brunswick, New Jersey \\ (b) Clark University, Worcester, Massachusetts \\ (c) University of Saskatchewan, Saskatchewan, Canada \\ (d) EMSL, Richland, Washington \\ (e) Pacific Northwest National Laboratory, Richland, Washington
}

Mercury was spilled and released as waste to the soil and groundwater at nuclear weapons production sites. In sediments with low levels of oxygen, bacteria can add a methyl group to the mercury, creating the neurotoxic substance methylmercury. To understand the formation of methylmercury, scientists need accurate information from this and related studies on the geochemistry of mercury.

Used in separating isotopes at U.S. nuclear weapons production sites, tons of mercury were released to the soil and groundwater. In anoxic or low-oxygen sediments, bacteria transform this silvery element when in the form of $\mathrm{Hg}(\mathrm{II})$ into a neurotoxin by adding a methyl group $\left(-\mathrm{CH}_{3}\right)$. The resulting methylmercury is a potent neurotoxic substance that accumulates in fish, which are harvested for food.

In a recent study, published in Environmental Science \& Technology and highlighted in Science, researchers from Rutgers University, EMSL, and Pacific Northwest National Laboratory showed that a simple iron mineral transforms $\mathrm{Hg}(\mathrm{II})$ to gaseous $\mathrm{Hg}(0)$. The mineral known as magnetite or $\mathrm{Fe}(\mathrm{II}) / \mathrm{Fe}(\mathrm{III})$ mixed valence iron oxide turns $\mathrm{Hg}(\mathrm{II})$ into $\mathrm{Hg}(0)$ in just minutes. Subsurface bacteria cannot add a methyl group to the gaseous mercury, so it does not become the neurotoxic methylmercury (Figure 1). 
The researchers conducted kinetic experiments at Rutgers University. These experiments showed that the $\mathrm{Hg}(\mathrm{II})$ gains electrons within minutes of entering an environment with magnetite. At EMSL, the scientists did Mössbauer spectroscopic analysis of reacted magnetite samples. The analysis revealed decreased $\mathrm{Fe}(\mathrm{II})$ in the magnetite. This decrease corresponds to the oxidation of $\mathrm{Fe}$ (II) to $\mathrm{Fe}(\mathrm{III})$ coupled to the reduction of $\mathrm{Hg}(\mathrm{II})$ to $\mathrm{Hg}(0)$. Finally, they used X-ray photoelectron spectroscopy to detect $\mathrm{Hg}$ (II) on magnetite surfaces. This analysis implied that adsorption is involved in the electron transfer process.

The results of these analysis suggest that the reaction of $\mathrm{Hg}$ (II) with the $\mathrm{Fe}(\mathrm{II})$-containing oxide is a favorable pathway. This research helps scientists understand and eventually control the creation of toxic methyl mercury. This research

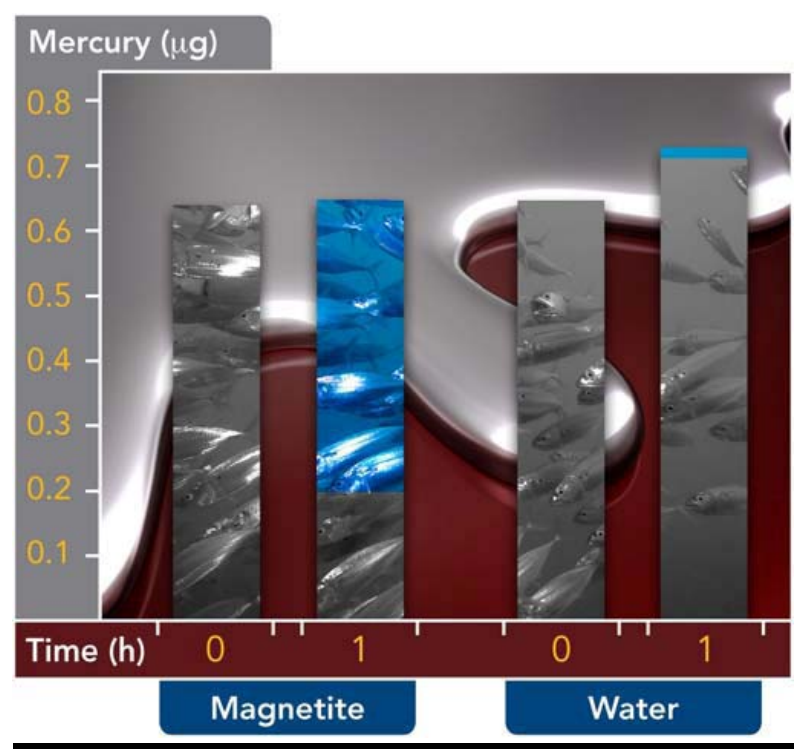

Figure 1. Magnetite reduces the amount of mercury in solution. This transformation makes the mercury unavailable for conversion by bacteria to a toxin. suggests that mineral-associated processes, such as adsorption and phase transformation, may provide stiff competition in mediating mercury's fate and mobility. This research was funded by DOE's Office of Biological and Environmental Research's Environmental Remediation Sciences Program.

\title{
Citation:
}

Wiatrowski HA, S Das, R Kukkadapu, ES Ilton, T Barkay, and N Yee. 2009. "Reduction of $\mathrm{Hg}(\mathrm{II})$ to $\mathrm{Hg}(0)$ by Magnetite.” Environmental Science \& Technology 43(14):5307-5313. DOI: 10.1021/es9003608

\section{Oxidative Dissolution Potential of Biogenic and Abiogenic $\mathrm{TcO}_{2}$ in Subsurface Sediments}

\author{
JK Fredrickson, ${ }^{\text {(a) }}$ JM Zachara, ${ }^{\text {(a) }}$ AE Plymale, ${ }^{\text {(a) }}$ SM Heald, ${ }^{\text {(b) }}$ JP McKinley, ${ }^{\text {(a) }}$ DW \\ Kennedy, ${ }^{\text {(a) }}$ C Liu, ${ }^{\text {(a) }}$ and P Nachimuthu ${ }^{(c)}$ \\ (a) Pacific Northwest National Laboratory, Richland, Washington \\ (b) Argonne National Laboratory, Argonne, Illinois \\ (c) EMSL, Richland, Washington
}

Subsurface contaminants such as ${ }^{99}$ Tc pose serious health risks. A study conducted by EMSL users and researchers helps provide a better understanding of the biogeochemical processes influencing the fate and transport of ${ }^{99}$ Tc and may lead to better predictions of its migration in the subsurface and new insights into remediation strategies at nuclear facilities worldwide.

Technetium-99 is a metal formed during nuclear reactions and can be released into the environment during nuclear fuels reprocessing. It is highly mobile in its most oxidized state, $\mathrm{Tc}(\mathrm{VII})$, and much less mobile in its reduced state, Tc(IV). Technetium contamination of groundwater is a chief concern at the Department of Energy's Hanford Site, where ${ }^{99} \mathrm{Tc}$ has leaked from nuclear waste storage tanks to depths of 60 meters in the vadose zone, which is the region above the water table. Using resources at the DOE's 
EMSL, researchers found that ${ }^{99} \mathrm{Tc}$ in natural subsurface sediments that has been reduced by Fe(II) associated with certain mineral phases may be exceedingly resistant to oxidation and, therefore, remobilization.

The research team, comprising scientists from Pacific Northwest National Laboratory and Argonne National Laboratory, examined two sediments that differed in mineralogy and aggregation state. One was obtained from the upper Ringold Formation near the Hanford Site and the other from the Field Research Center (FRC) at the Oak Ridge Site. Both sediments contained Fe(III) and Mn(III/IV) as redox active phases, although the FRC sediment also contained different Fe-phyllosilates minerals. The researchers used a Shewanella, a metal-reducing bacterium, to reduce manganese and iron oxides in the sediments. Iron in the bioreduced sediments, in turn, reduced Tc(VII) to Tc(IV). During the subsequent oxidation phase, the researchers measured Tc release from the sediments and found that $\mathrm{Tc}(\mathrm{IV})$ in the Ringold Formation sediment was oxidized and released much more rapidly and extensively than in the FRC sediment. The researchers used tools at EMSL and the Advanced Photon Source at Argonne National Laboratory to characterize sediment-associated ${ }^{99} \mathrm{Tc}$ and then used EMSL's X-ray microprobe, electron microprobe, X-ray absorption spectroscopy, and micro X-ray diffraction to analyze the FRC sediment and Tc-containing particles isolated from the sediment (Figure 1). These analyses revealed an oxidationresistant Tc(IV) phase that exists with $\mathrm{Fe}(\mathrm{III})$ within iron-containing micas in the FRC sediment. The mica particles were a mineral phase called celadonite.
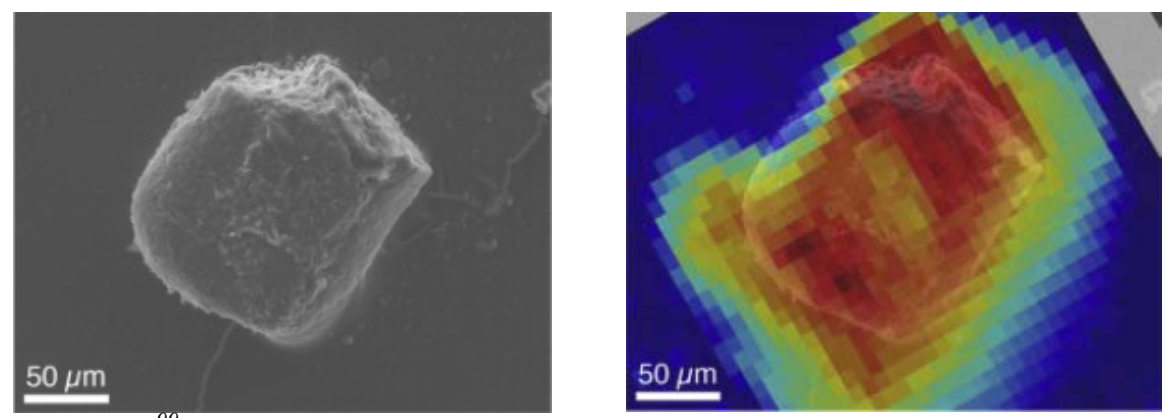

Figure 1. ${ }^{99}$ Tc-containing particle isolated from oxidized FRC sediment:

backscattered electron micrograph (left) and electron microprobe energy dispersive $X$-ray analysis (right).

This work offers insights into electron exchange at the microbe-mineral interface and its effect on the fate and transport of subsurface contaminants. This research was performed as part of EMSL's

Biogeochemistry Grand Challenge and was supported by DOE's Office of Biological and Environmental Research Program. It was featured in Geochimica et Cosmochimica Acta.

\section{Citation:}

Fredrickson JK, JM Zachara, AE Plymale, SM Heald, JP McKinley, DW Kennedy, C Liu, and P Nachimuthu. 2009. "Oxidative Dissolution Potential of Biogenic and Abiogenic TcO2 in Subsurface Sediments.” Geochimica et Cosmochimica Acta 73(8):2299-2313. 


\section{Science of Interfacial Phenomena}

Interfaces control many chemical and physical properties of natural and engineered materials critical to environmental- and energy related research and technologies important to the mission of DOE and to society in general. The importance of interfaces has been highlighted in DOE workshops covering topics including geosciences, solid-state lighting, solar energy, and advanced nuclear energy systems.

Tailored or designed surfaces and interfaces are important as model systems for detailed study of processes that occur on natural heterogeneous materials in the environment and for developing materials with new properties for energy production, catalysis, or other uses.

The behaviors of complex heterogeneous materials in the environment may never be fully understood without model systems that allow specific aspects of that complexity to be examined in detail. Likewise, material systems with interfaces optimized with specific properties are essential for the technologies needed for a secure environment and a stable energy future. Understanding these often complex interfaces requires methods to characterize naturally complex materials and minerals found in the environment and to understand increasingly complex materials designed and synthesized to have a desired functionality.

The Science of Interfacial Phenomena science theme is focused on understanding and gaining control of structure-function relationships at the atomic level to allow the design of new energy technologies and to understand the behaviors of natural systems. Topics of major emphasis in this science theme include the following:

- Catalytic structure-function relationships to allow precise control of catalytic activity and selectivity.

- Gaining critical knowledge of photocatalysis and photochemistry.

- Design material systems with specialized charge and mass transport properties.

The science and technological issues that need to be addressed to achieve these goals complement and to some degree naturally intersect those of the other science themes. The ability to characterize surfaces and small particles also provides a natural pathway for addressing issues of atmospheric aerosol chemistry. Developing technologies that rely on improved understanding and control of molecular-level structural, dynamic, and transport properties of interfaces include the following:

- New generations of selective catalysts

- Thin-film solar cells

- Solid-state lighting

- Hydrogen production and storage

- Solid-oxide fuel cells

- Chemical sensors and radiation detectors

- Materials for next-generation nuclear reactors. 


\title{
Comparative Analysis of Urban Atmospheric Aerosol by Particle- Induced X-Ray Emission (PIXE), Proton Elastic Scattering Analysis (PESA), and Aerosol Mass Spectrometry (AMS)
}

\author{
KS Johnson, ${ }^{(a),(b)}$ A Laskin, ${ }^{(c)}$ JL Jiminez, ${ }^{(d)}$ V Shutthanandan, ${ }^{(c)}$ LT Molina, ${ }^{(a),(e)}$ \\ $D$ Salcedo, ${ }^{(f)} K$ Dzepina, ${ }^{(d)}$ and MJ Molina ${ }^{(a),(g)}$ \\ (a) Massachusetts Institute of Technology, Cambridge, Massachusetts \\ (b) Finnegan, Henderson, Farabow, Garrett, and Dunner, LLP, Washington, D.C. \\ (c) EMSL, Richland, Washington \\ (d) University of Colorado, Boulder, Colorado \\ (e) Molina Center for Energy and the Environment, La Jolla, California \\ (f) Universidad Autonoma del Estado de Morelos, Cuernavaca, Morelos, Mexico \\ (g) University of California, San Diego, La Jolla, California
}

EMSL researchers and users have conducted the first comparison of data sets obtained from protoninduced X-ray emission (PIXE) and proton elastic scattering analysis (PESA) to aerosol mass spectrometry (AMS) aerosol measurements, constituting, to the researchers' knowledge, the first report of PESA hydrogen fraction measurements in urban organic aerosols. This multidisciplinary research can help researchers more accurately interpret data from large, expensive aerosol studies and is vital in devising strategies to reduce the impact of aerosols on the climate.

A multidisciplinary team from Massachusetts Institute of Technology, EMSL, University of Colorado, Molina Center for Energy and the Environment, and Universidad Autonoma del Estado de Morelos in Mexico published the first comparison of data sets obtained from proton-induced X-ray emission (PIXE) and proton elastic scattering analysis (PESA) to aerosol mass spectrometry (AMS) measurements. The team compared the results of PIXE and PESA, both at EMSL, with AMS results (Figure 1). PIXE, PESA, and AMS use different methods of analysis yet provided complementary data sets. By utilizing the strengths of each instrument, scientists can more accurately interpret the results of studies on air pollution, an important issue for human health and environmental protection.

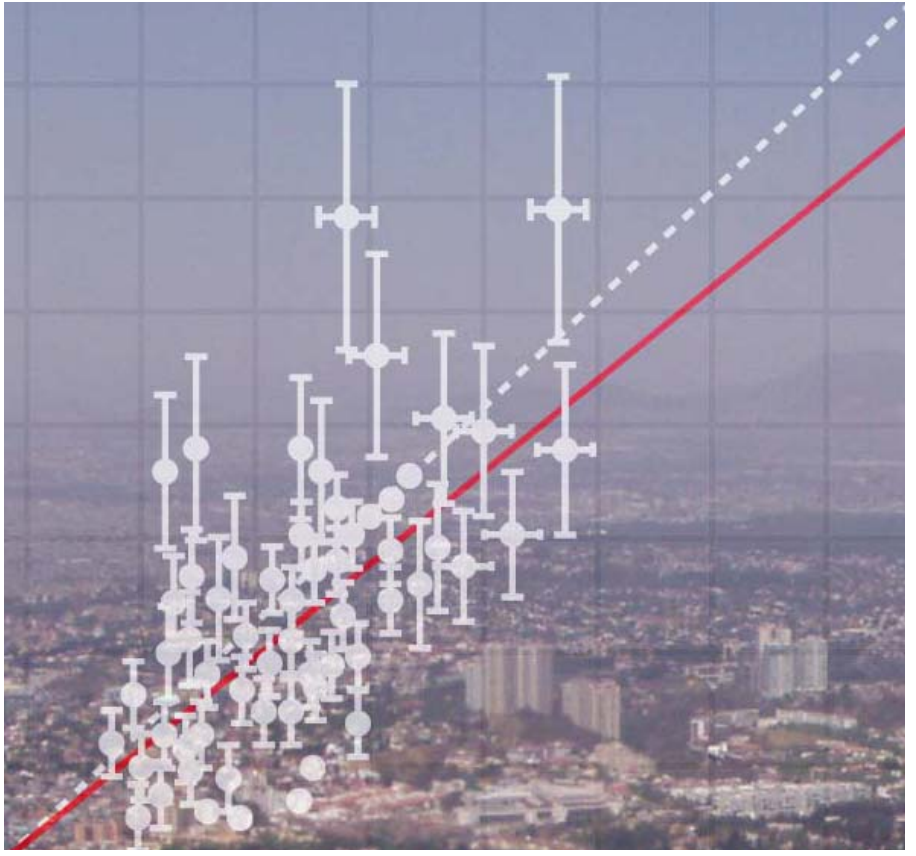

Figure 1. Complementary capabilities of PIXE, PESA, and AMS measurements were used to provide comprehensive data for analysis of atmospheric aerosols in Mexico City.

As part of the novel study, the team found good agreement between the sulfur measurements from PIXE and AMS. This comparison will allow scientists to vet field results with laboratory measurements and vice versa. Also, they found discrepancies between the hydrogen mass fractions assessed from PESA and AMS measurements. The discrepancy was attributed to the presence of nonvolatile organic compounds in 
aerosol samples. These compounds were not vaporized in the PESA and therefore were detected by the PESA method, while AMS detects organic aerosols of relatively high volatility. The study highlights the fact that complementary aerosol analysis capabilities can be used in the same environmental setting to provide comprehensive information about the amount of volatile and nonvolatile particles in the air.

This research, funded by the Mexican Metropolitan Environmental Commission, National Science Foundation, Environmental Protection Agency, and DOE, was published in Environmental Science and Technology.

\title{
Citation
}

Johnson KS, A Laskin, JL Jiminez, V Shutthanandan, LT Molina, D Salcedo, K Dzepina, MJ Molina. 2008

"Comparative Analysis of Urban Atmospheric Aerosol by Particle-Induced X-ray Emission (PIXE), Proton Elastic Scattering Analysis (PESA), and Aerosol Mass Spectrometry (AMS)." Environmental Science and Technology 42(17):6619-6624 .

\section{Understanding How Surface Morphology and Hydrogen Dissolution Influence Ethylene Hydrogenation on Palladium}

\author{
Z Dohnálek, ${ }^{(a)} \mathbf{J ~ K i m , { } ^ { ( a ) }}$ and BD Kay ${ }^{(a)}$ \\ (a) Pacific Northwest National Laboratory, Richland, Washington
}

EMSL users have discovered that the arrangement of palladium atoms can improve the metal's ability to speed reactions. The team from Pacific Northwest National Laboratory designed a catalyst so that the palladium atoms on the catalyst surface had plenty of hydrogen atoms nearby but few other palladium atoms. When they tested the catalyst, it added hydrogen to 50 percent of the target hydrocarbons, an order-of-magnitude improvement over unmodified palladium.

Palladium (Figure 1, palladium surface) is an expensive industrial catalyst, costing about $\$ 180$ an ounce. Designing the catalyst so that it performs better could mean less palladium is needed and more of the desired products are generated. This atom-by-atom design of the catalyst could open doors for controlling the physical and chemical properties of this important material.

At EMSL, the team began by depositing palladium atoms at -420 Fahrenheit. To deposit the atoms, they placed a substrate at an oblique angle to make an extremely porous palladium film. They characterized the surface morphology using a combination of scanning electron microscopy and low-temperature physisorption techniques afforded by EMSL's suite of state-of-the-art instrumentation. The team saw that the deposition caused the atoms to be arranged in peaks and valleys.

They tested the catalyst with a hydrogenation reaction, converting ethylene to ethane by adding hydrogen. They discovered that the tailored catalyst was more efficient than previous studies suggested. Typically, when palladium catalyzes the hydrogenation reaction, less than 1 percent of the ethylene is converted. Now, 50 percent of the ethylene was converted. 
Based on their observations of high catalytic activity, the team embarked on additional experiments. They grew thin layers of palladium on a nonporous substrate, creating a smooth surface. Then, they added a few extra palladium atoms on top.

Again, the researchers noted the catalyst's improved efficiency. They analyzed the results and determined the catalyst worked for two reasons. First, the palladium atoms on the surface were unencumbered by nearby palladium atoms. Second, the thin films prevented the hydrogen atoms from

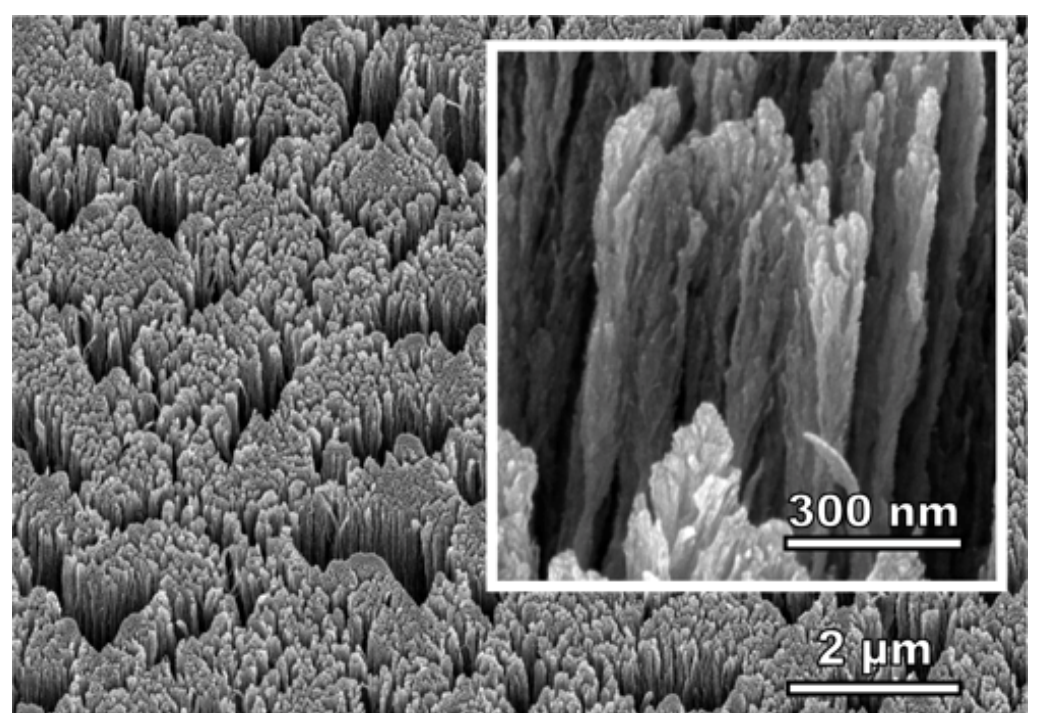

Figure 1. Palladium surface. diffusing into the bulk of the material. The thin films kept the hydrogen at the surface, where it could be used. Past studies did not see these results because thicker crystals soaked up the hydrogen.

The researchers are now continuing to provide new insights into the behavior of catalysts that are of value to industrial and energy applications.

This research, funded by DOE's Office of Basic Energy Sciences, Chemical Sciences Division and which is part of PNNL's Institute for Interfacial Catalysis, was featured in the Journal of Physical Chemistry C.

\title{
Citation
}

Dohnálek Z, J Kim, and BD Kay. 2008. "Understanding How Surface Morphology and Hydrogen Dissolution Influence Ethylene Hydrogenation on Palladium." Journal of Physical Chemistry C 112(40):15796-15801.

\section{Vacancy-Assisted Diffusion of Alcoxy Species on Rutile $\mathrm{TiO}_{2}(110)$}

\author{
Z Zhang, ${ }^{(a)}$ R Rousseau, ${ }^{(a)} \mathrm{J} \mathrm{Gong}^{\left({ }^{(b)}\right.} \mathrm{SC} \mathrm{Li,}{ }^{(b)} \mathrm{BD} K a y,{ }^{(a)} \mathrm{Q} \mathrm{Ge},{ }^{(c)}$ and Z Dohnálek ${ }^{(a)}$ \\ (a) Pacific Northwest National Laboratory, Richland, Washington \\ (b) University of Texas at Austin, Austin, Texas \\ (c) Southern Illinois University, Carbondale, Illinois
}

This study is the first to show how the vacancies on the surface of a titanium dioxide catalyst can aid in moving molecules. Learning how atoms behave on the surface of the catalyst could help tailor molecular delivery systems. For example, scientists could design a vacancy-rich system to lead the desired portions of an alcohol to sites where they are converted into hydrogen fuel and carbon dioxide. In addition, this atom-by-atom understanding can help in designing or refining technologies that use titanium dioxide, such as water purifiers, self-cleaning glass, and air purifiers. 
On the surface of a common catalyst, alcohol molecules do the unexpected: they hop with the help of defects, according to a team of EMSL users from the Pacific Northwest National Laboratory, University of Texas at Austin, and Southern Illinois University. The catalyst's surface has holes or vacancies where an oxygen atom should be, but isn't. Instead of ignoring these vacancies, the alcohol discards the hydrogen from its only oxygen and jumps into the vacancy. Then, the molecule hops from one vacancy to the next as they come nearby.

The researchers combined experiment and theory at EMSL to study the behavior of alcohol on rutile titanium dioxide (Figure 1). For the experiments, they used imperfect titanium dioxide with holes or vacancies on the surface where oxygen atoms should be. Then, they added a 4-carbon alcohol, called 2butanol.

Using a state-of-the-art scanning tunneling microscope, they found that the alcohol did not behave as expected. The alcohol shed its hydroxyl hydrogen (see sidebar), leaving the oxygen exposed. The oxygen, still attached to the alcohol's carbons and hydrogens, jumped into the nearest hole. But, the oxygen did not stay put for long. It hopped from vacancy to vacancy as the vacancies came nearby.

Next, the team analyzed these results with detailed theoretical calculations. They found that this travelling behavior at low temperatures required less energy than the other route: breaking off the whole hydroxyl group and moving the hydrocarbon along the surface. To confirm these results, the team studied a range of alcohols, one to eight carbons in length. They found the same pattern of movement.

The team is now moving on to determine how other molecules would break and diffuse on more active oxides, such as tungsten trioxide.

The research was supported by DOE's Office of Basic Energy Sciences, Chemical and Material Sciences Division, the Robert A. Welch Foundation, the National Science Foundation, and PNNL's Summer Research Institute. Results were featured in Physical Review Letters.

\section{Citation}

Zhang Z, R Rousseau, J Gong, SC Li, BD Kay, Q Ge, and Z Dohnálek. 2008. "Vacancy-assisted Diffusion of Alkoxy Species on Rutile $\mathrm{TiO}_{2}(110)$.” Physical Review Letters 101:156103. 


\title{
Self-Assembly of Cerium Oxide Nanostructures in Ice Molds
}

\author{
AS Karakoti, ${ }^{(a)}$ SVNT Kuchibhatla, ${ }^{(a),(b)}$ DR Baer, ${ }^{(b)} S$ Thevuthasan, ${ }^{(b)}$ DC Sayle, ${ }^{(c)}$ and \\ S Seal ${ }^{(a)}$ \\ (a) University of Central Florida, Orlando, Florida \\ (b) EMSL, Richland, Washington \\ (c) Defence Academy of the United Kingdom, Swindon, United Kingdom
}

Defining the conditions under which nanoparticles can be made to self-assemble into desired geometries opens new doors for nanomaterials research. In addition, many existing processes to manufacture nanomaterials use high molecular weight surfactants and solvents such as toluene, often generating chemical waste. EMSL staff and collaborators have demonstrated a new method to produce nanostructures that minimizes harm to the environment in a cost-effective manner. Nanomaterials produced with the research teams' new water-based method are less expensive and could drive technological advancements in a variety of nano-fields.

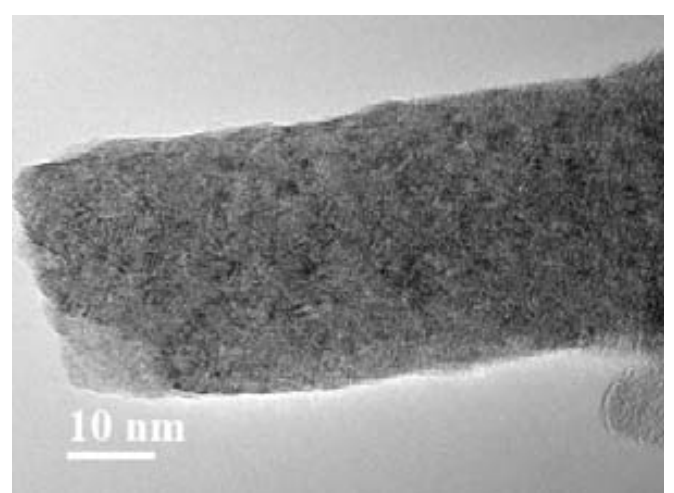

Figure 1. Transmission electron microscopy image of a cerium oxide nanorod formed in an ice channel.

EMSL researchers and their collaborators have tested a new way to build nanostructures that is "green" and elegantly simple. Taking a cue from nature, collaborators from EMSL, the University of Central Florida, and Defence Academy of the United Kingdom grew cerium oxide nanostructures inside the tiny voids that form in aqueous solutions upon freezing. By controlling solution freezing rate, nanoparticle concentration, and storage temperature, the team's ice mold method may be used to produce nanostructures with tailored shapes and sizes that have a myriad of applications - from biology to electronics.

The team's green chemical method was made possible by two natural phenomena: solute rejection and self-assembly. Upon freezing, aqueous solutions force out, or reject, impurities because they cannot be accommodated in the rigid lattice structure of ice. Depending on the freezing conditions, ice does not form a perfect lattice throughout. Rather, it has tiny pockets and channels. It is into these voids that impurities, in this case cerium oxide nanoparticles, become trapped together and can self-assemble.

Upon freezing cerium oxide nanoparticles in solution at different temperatures and rates and storing the frozen solution for days to weeks, the research team used transmission electron microscopy and other tools to characterize the resulting nanostructures (Figure 1). They observed that cerium oxide nanoparticles trapped in channels in the ice formed nanorods, and those trapped in larger voids formed octahedral superstructures. The team's experimental observations were consistent with molecular dynamics simulations of nanoparticle behavior under geometrically constrained conditions.

This research, supported by the National Science Foundation, was featured in Small.

\section{Citation}

Karakoti AS, SVNT Kuchibhatla, DR Baer, S Thevuthasan, DC Sayle, and S Seal. 2008. "Self-Assembly of Cerium Oxide Nanostructures in Ice Molds." Small doi:10.1002/smll.200800219. The work was also highlighted in Nature Nanotechnology, doi:10.1038/nnano.2008.259. 


\section{Intrinsic Diffusion of Hydrogen on Rutile $\mathrm{TiO}_{2}(110)$}

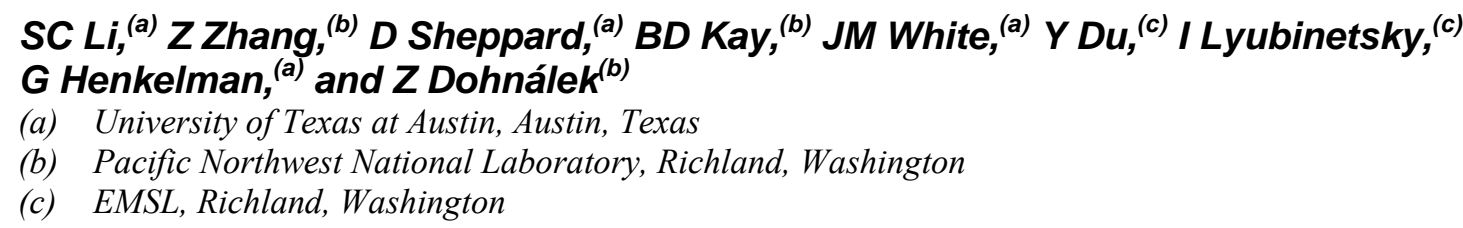

(b) Pacific Northwest National Laboratory, Richland, Washington

(c) EMSL, Richland, Washington

Understanding what happens on the surface of rutile titanium dioxide could help researchers tailor this material to use sunlight to speed the reaction that splits water into hydrogen and oxygen. The resulting hydrogen can be used to power fuel cells that could replace gasoline-powered engines in cars and trucks.

When water breaks apart on a well-known catalyst, the once-close hydrogen atoms quickly part company in a complicated process, according to scientists at Pacific Northwest National Laboratory and the University of Texas at Austin. Their detailed study shows the hydrogen atoms are slightly repelled by each other on the surface of the rutile titanium dioxide catalyst. The atoms move apart by first sliding an electron over to their new home. Then, the rest of the atom follows.

The research team took a two-prong approach to studying hydrogen movement or diffusion. They began with experimental studies and followed up with theoretical calculations. For the experiments, they used temperature-dependent measurements to track hydrogen movement on the catalyst's surface. They performed these measurements using one of EMSL's scanning tunneling microscopes that can resolve single atoms on a surface (Figure 1). For the theoretical studies, they used density functional theory calculations and other models.
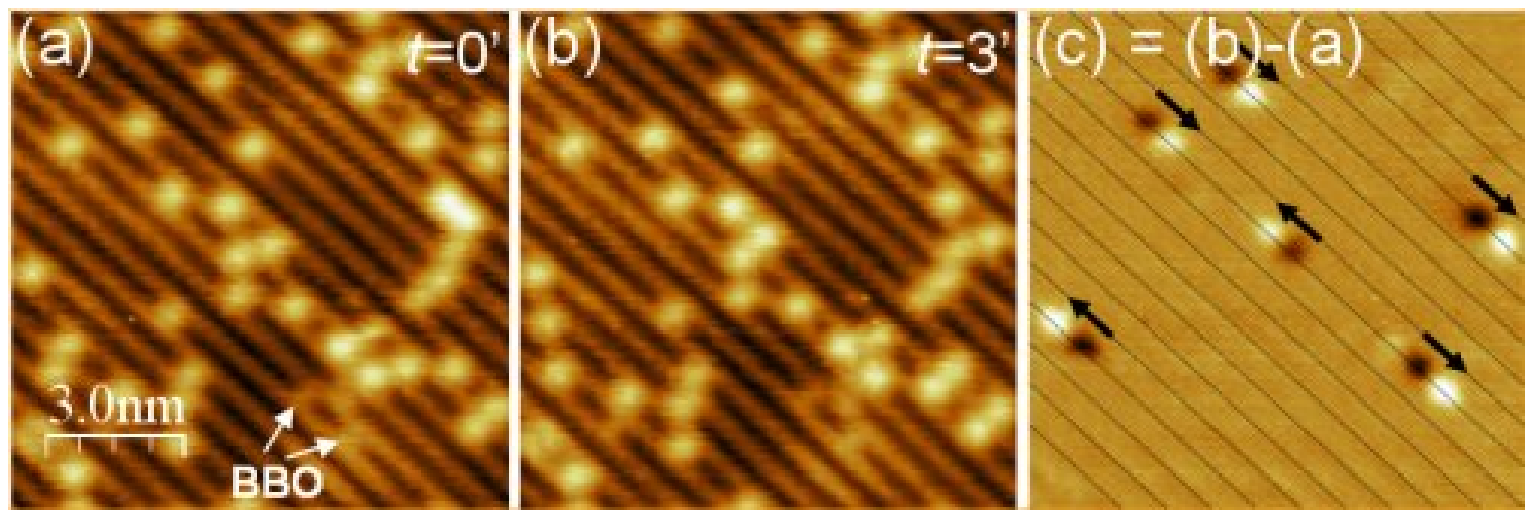

Figure 1. Hydrogen movement shown by the scanning tunneling microscope. By taking snapshots before (left) and after (middle) of the motion of the hydrogen atoms on the catalyst, the scientists can determine where and how fast the atoms moved (final).

The results from the experimental and theoretical studies did not agree. While both show hydrogen diffusing across the surface at the same rates, the underlying parameters controlling the rates differed.

The researchers believed the differing results were because of how the hydrogen moves. They speculated that the hydrogen diffused via a two-step process. A hydrogen atom has two parts: a positive nucleus and a negative electron. In diffusion, the lone electron nimbly hops over to the new location first. Then, the larger nucleus hefts itself to the new location. The first step, the electron moving, is not accounted for in the theoretical calculations. 
The team is continuing to study water on rutile titanium dioxide, focusing on its reactions with oxygen to get one step closer to understanding titanium dioxide as a water splitting catalyst.

The research was funding by DOE's Office of Basic Energy Sciences, Chemical Sciences; the Robert A. Welch Foundation; and National Science Foundation. It was featured in the Journal of the American Chemical Society.

\title{
Citation
}

Li SC, Z Zhang, D Sheppard, BD Kay, JM White, Y Du, I Lyubinetsky, G Henkelman, and Z Dohnálek. 2008. "Intrinsic Diffusion of Hydrogen on Rutile $\mathrm{TiO}_{2}(110)$." Journal of the American Chemical Society

130(28):9080-9088.

\section{Photoelectron Spectroscopic and Theoretical Study of $\mathrm{B}_{16}{ }^{-}$and $\mathrm{B}_{16}{ }^{2-}$ : An All-Boron Naphthalene}

\author{
AP Sergeeva, ${ }^{(a)}$ DY Zubarev, ${ }^{(a)}$ HJ Zhai, ${ }^{(b)(c)}$ Al Boldyrev, ${ }^{(a)}$ and LS Wang ${ }^{(b)(d)}$ \\ (a) Utah State University, Logan, Utah \\ (b) Washington State University-Tri-Cities, Richland, Washington \\ (c) EMSL, Richland, Washington \\ (d) Pacific Northwest National Laboratory, Richland, Washington
}

Engineering technologies to solve energy or security issues benefit from knowledge of the atomic-level structure of materials such as highly reactive boron. Researchers from Utah State University, Washington State University, and Pacific Northwest National Laboratory have discovered the atomic structure of two boron clusters: $B_{16}{ }^{-}$and $B_{16}{ }^{2-}$.

Using EMSL's laser vaporization and time-of-flight mass spectrometry capabilities, a researcher team from Utah State University, Washington State University, and Pacific Northwest National Laboratory produced $\mathrm{B}_{16}$ clusters and examined them using photoelectron spectroscopy. They did theoretical calculations to compare with the experimental data and determined the cluster's structure and chemical bonding. Molecular orbital analysis indicated that $\mathrm{B}_{16}{ }^{2-}$ possesses $10 \pi$ electrons and a $\pi$ bonding pattern similar to naphthalene, and it can be viewed as an all-boron version of the aromatic organic molecule used in mothballs (Figure 1).

This research provides detailed knowledge about the structure and chemical bonding in $\mathrm{B}_{16}$ clusters, information that was not known previously. This work furthers the scientific basis for the development of novel boron nanostructures. In addition, fundamental insights into the structure of highly reactive boron
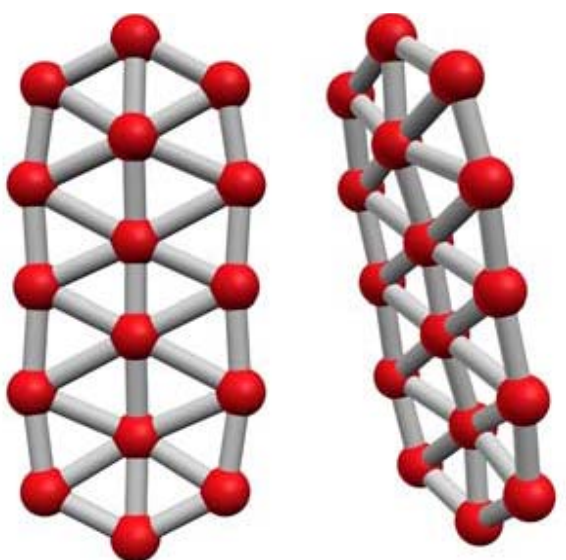

Figure 1. The structures of two 16-atom boron clusters were recently discovered by EMSL users. The $B_{16}{ }^{2-}$ structure (right) has $10 \pi$ electrons and $a \pi$ bonding pattern similar to naphthalene. clusters provide foundational information that other researchers can build on. This information could help the design of new boron-based nanomaterials for energy or security applications. 
The research, supported by the National Science Foundation, was published in the Journal of the American Chemical Society.

\title{
Citation
}

Sergeeva AP, DY Zubarev, HJ Zhai, AI Boldyrev, and LS Wang. 2008. "Photoelectron Spectroscopic and Theoretical Study of $\mathrm{B}_{16}{ }^{-}$and $\mathrm{B}_{16}{ }^{2-}$ : An All-Boron Naphthalene." Journal of the American Chemical Society 130(23):7244-7246.

\section{Imaging Consecutive Steps of $\mathrm{O}_{2}$ Reaction with Hydroxylated $\mathrm{TiO}_{2}(110)$ : Identification of $\mathrm{HO}_{2}$ and Terminal $\mathrm{OH}$ Intermediates}

\author{
Y Du, ${ }^{(a)}$ NA Deskins, ${ }^{(b)} Z$ Zhang, ${ }^{(b)} Z$ Dohnalek, ${ }^{(b)}$ M Dupuis, ${ }^{(b)}$ and I Lyubinetsky ${ }^{(a)}$ \\ (a) EMSL, Richland, Washington \\ (b) Pacific Northwest National Laboratory, Richland, Washington
}

Expected but elusive-that's how scientists described two intermediates (Figure 1) that may play an important role in the reaction that turns water into hydrogen. Now, thanks to the work of six researchers at EMSL and Pacific Northwest National Laboratory, these intermediates are no longer a mystery. In a recent article featured on the cover of the Journal of Physical Chemistry $\mathrm{C}$, the researchers provided images of two intermediates that occur during the reaction that changes water into hydrogen.
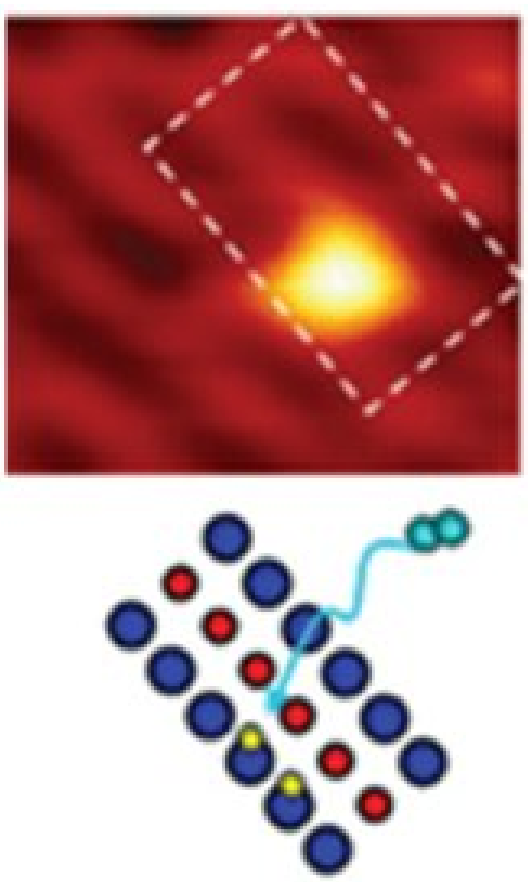
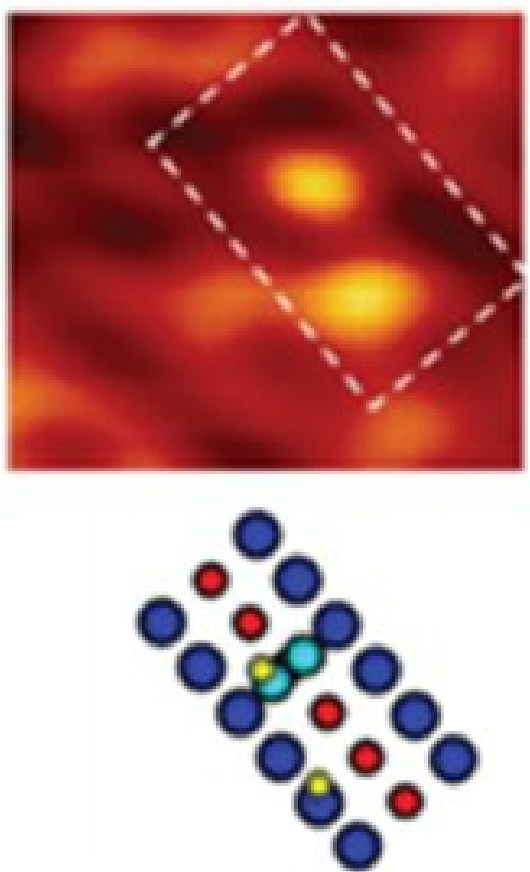
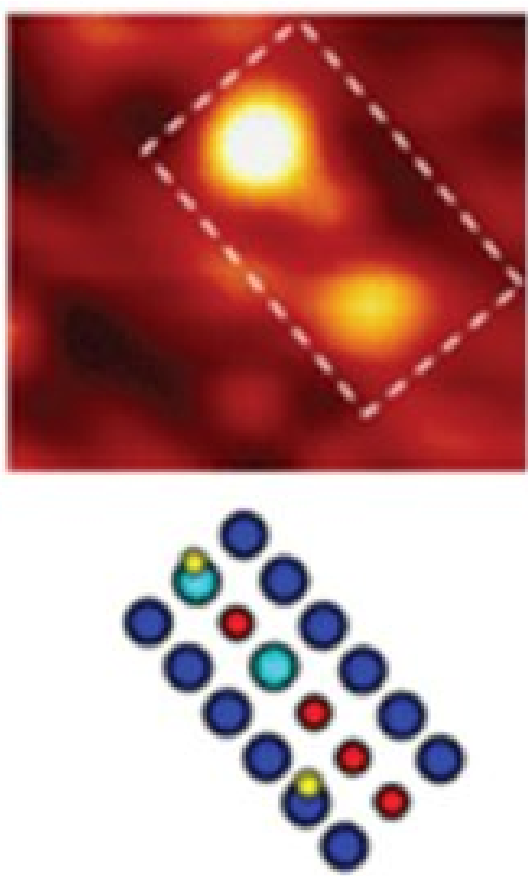

Figure 1. Two intermediates were discovered in the conversion of water to hydrogen (top: scanning tunneling microscope images). Forming these intermediates begins with oxygen (aqua). It snuggles in between the raised oxygen rows (blue), pulling a nearby hydrogen (yellow) on top of it. Later, this intermediate can split apart, creating oxygen and hydrogen (aqua and yellow) bound together and bound to a titanium atom (red).

An intermediate is a transitory product created as a chemical reaction moves from its starting materials to the final product. Some reactions have a few intermediates, others hundreds. 
For a long time, people expected some intermediate should be formed during the reaction of oxygen and hydrogen on titanium dioxide. It was expected, but nobody had seen it. Until now. Also, the team found that they could follow all of the steps that occur during oxygen and hydrogen transformation into water.

Discovering the intermediates and uncovering the intricate five-step process may shed light on the catalyzed reactions necessary to mass produce hydrogen. This simple molecule could power fuel cells that provide cleaner, quiet sources of energy.

Fuel cells are in use today, powering large buildings and tiny sensors and computers. Large stationary fuel cells provide backup power to hospitals, nursing homes, and other buildings. They also power remote outposts that do not have access to electricity. In vehicles, smaller cells are replacing or supplementing fossil fuel in cars, trucks, planes, trains, and boats.

The intricacy of the reactions and an exceptionally small number of molecules involved has prevented finding the intermediates until now. To combat these challenges, the team combined experimental techniques with theoretical techniques and computations. They prepared a partially hydroxylated, reduced titanium dioxide catalyst at room temperature so that the surface contained both hydroxyl groups and oxygen vacancies. Next, they exposed the catalyst to oxygen and recorded the reactions. These reactions were imaged using the state-of-the-art scanning tunneling microscope at EMSL. This instrument, available to users from around the world, can show what single molecules and atoms are doing on catalytic surfaces.

Once the team's experimental experts acquired the images, the theorists went to work. Using EMSL computational resources as well as those at the National Energy Research Scientific Computing Center, they performed density-functional-theory calculations to help interpret the images. Based on the theory and experimentation, the team reported the first observed adsorbed hydroperoxyl. Hydroperoxyl is two oxygen atoms bound together and also containing a single hydrogen atom. The hydroperoxyl is attached to the surface, with a single bond forming between one of the oxygen atoms and a titanium atom.

The hydroperoxyl can then break apart. One of the oxygen atoms stays bound to the titanium atom. The remaining oxygen and hydrogen, still bound together, bounce down to bind to another titanium atom. This oxygen-hydrogen combination is the second elusive intermediate: a terminal hydroxyl group on the catalyst surface.

The team will continue to explore catalysts and the reactions involved in producing hydrogen from water and sunlight. Their next paper, to be published soon in a prominent journal, looks at another variation, when the starting catalyst surface has oxygen adatoms.

The research is supported by DOE's Office of Basic Energy Sciences.

\section{Citation}

Du Y, NA Deskins, Z Zhang, Z Dohnalek, M Dupuis, and I Lyubinetsky. 2009. "Imaging Consecutive Steps of $\mathrm{O}_{2}$ Reaction with Hydroxylated $\mathrm{TiO}_{2}(110)$ : Identification of $\mathrm{HO}_{2}$ and Terminal $\mathrm{OH}$ Intermediates." Journal of Physical Chemistry C 113(2):666-671. 


\title{
Two Pathways for Water Interaction with Oxygen Adatoms on $\mathrm{TiO}_{2}(110)$
}

\author{
Y Du, ${ }^{(a)}$ NA Deskins, ${ }^{(b)} Z_{\text {Zhang, }}{ }^{(b)} Z$ Dohnalek, ${ }^{(b)}$ M Dupuis, $^{(b)}$ and I Lyubinetsky ${ }^{(a)}$ \\ (a) EMSL, Richland, Washington \\ (b) Pacific Northwest National Laboratory, Richland, Washington
}

Single oxygen atoms dancing on a metal oxide slab, glowing brighter here and dimmer there, have helped chemists better understand how water splits into oxygen and hydrogen. In the process, the scientists have visualized a chemical reaction that had previously only been talked about. The new work improves our understanding of the chemistry needed to generate hydrogen fuel from water or to clean contaminated water.

A research team from EMSL and Pacific Northwest National Laboratory made the discovery while trying to determine the basics of how titanium dioxide -- a compound sometimes found in sunscreen -- breaks down water. The chemical reactions between water and oxygen are central to such varied processes as hydrogen production, breaking down pollutants, and in solar energy.

While exploring titanium dioxide as a way to split water into its hydrogen and oxygen pieces, researchers can use scanning tunneling microscopy to watch the chemical reaction. The surface of a slab of titanium dioxide is like a corn field: rows of oxygen atoms rise from a patch of titanium atoms. The alternating oxygen and titanium rows look like stripes.

Scientists can also see some atoms and molecules that come to rest on the surface as bright spots. One such visible atom is a single oxygen atom that comes to rest on a titanium atom, called an "adatom". Chemists can only see water molecules if they drop the temperature dramatically -- at ambient temperature, water moves too fast for the method to pick them up.

In this work, the research team studied water's reactions with titanium dioxide at ambient temperature at EMSL. Starting with a surface plated with a few oxygen adatoms, they added water -- and the adatoms started to dance, moving back and forth along the titanium row.

Remarkably, the adatoms didn't just slide up and down the stripes. They also bounced out of them and landed in others. Calculating how much energy it would take for the adatoms to move by themselves, much less hop over an oxygen row, the chemists suspected the adatoms were getting help -- most likely from the invisible water molecules.

To make sense of the dancing adatoms, the team calculated how much energy it would take to move adatoms with the help of water molecules. If a water molecule sits down next to an adatom, one of the water's hydrogen atoms can jump to the adatom, forming two oxygen-hydrogen pairs.

These pairs are known as hydroxyls and tend to steal atoms from other molecules, including each other. One of the thieving hydroxyls can then nab the other's hydrogen atom, turning back into a water molecule. The water molecule floats off, leaving behind an adatom. Half the time, that adatom is one spot over -- which makes the original appear to have moved.

The chemists determined that water can help the adatom jump a row as well: If a water molecule and an adatom are situated on either side of a raised oxygen row, a row oxygen can serve as the middleman, 
handing over a hydrogen from the water molecule to the adatom. Again, two hydroxyls form, one ultimately stealing both hydrogens (with the help of the middleman) and zipping away as water. If the incoming water molecule has been stripped, the adatom appears to have hopped over.

The calculated energy required for these different scenarios fit well with the team's experimental data. When a row oxygen serves as a middleman, the process is known as "pseudo-dissociation", a reaction suggested by chemists but until now, never verified experimentally.

In the future, the team plans on determining if water can make the adatoms move other species and more than one space at a time. In addition, they will investigate how light affects the reaction.

The research, supported by the Department of Energy's Office of Science, was published in Physical Review Letters.

\section{Citation}

Du Y, NA Deskins, Z Zhang, Z Dohnálek, M Dupuis, and I Lyubinetsky. 2009. "Two Pathways for Water Interaction with Oxygen Adatoms on $\mathrm{TiO}_{2}(110)$." Physical Review Letters DOI 10.1103/PhysRevLett.102.096102

\section{Macrophage Responses to Silica Nanoparticles are Highly Conserved Across Particle Sizes}

\section{KM Waters, ${ }^{(\text {a) }}$ LM Masiello, (a) $R C$ Zangar, ${ }^{(a)} B J$ Tarasevich, ${ }^{(a)} N J$ Karin, ${ }^{(a)}$ RD Quesenberry, ${ }^{(a)}$ S Bandyopadhyay, ${ }^{\text {(a) }}$ JG Teeguarden, ${ }^{\text {(a) }}$ JG Pounds, ${ }^{\text {(a) }}$ and BD Thrall ${ }^{(a)}$}

(a) Pacific Northwest National Laboratory, Richland, Washington

The increasing use of nanomaterials in products from semiconductors to sunscreen is revolutionizing products in many industries. This popularity results from significant changes in the chemical reactivity and physical properties of many materials significantly change at the nanoscale - that is, having at least one dimension of 100 nanometers or less. However, the same properties that make nanomaterials attractive for commercial and medical use may also enhance their biological reactivity, raising concerns about their potential toxicity. For example, materials classically considered biologically inert, such as amorphous silica, titanium dioxide and gold have been reported to cause exacerbated biological responses when used at the nanoscale.

Scientists are now closer to determining what a "safe dose" of nanomaterials is to humans, thanks to a comprehensive study published by EMSL users from the Pacific Northwest National Laboratory. Using an in vitro model of macrophage cells, they demonstrated that the ability of amorphous silica particlesakin to sand - to stimulate inflammation and toxicity in cells scales closely with the total particle surface area.

To the research team's knowledge, this is the first study to use genome-scale measurements to evaluate whether the cellular effects induced by nanomaterials are dependent on particle size. The results suggest that the surface chemistry properties of amorphous silica that are responsible for its bioactivity do not significantly change as particle size decreases to the nanoscale. 
The molecular mechanisms by which nanoparticles stimulate cellular responses are still poorly understood. Yet it is clear that particle surface chemistry is an important driving factor. By identifying the early transcriptional events and pathways activated by amorphous silica in macrophages - white blood cells that surround and kill invaders to the tissues - the research team's results significantly extend previous studies. Because of their role in immune surveillance, macrophages are important sentinels for biological response to pathogens.

To identify hazards and assess human risk from nanoparticle exposure, researchers must extrapolate dose-response data from animal studies to human
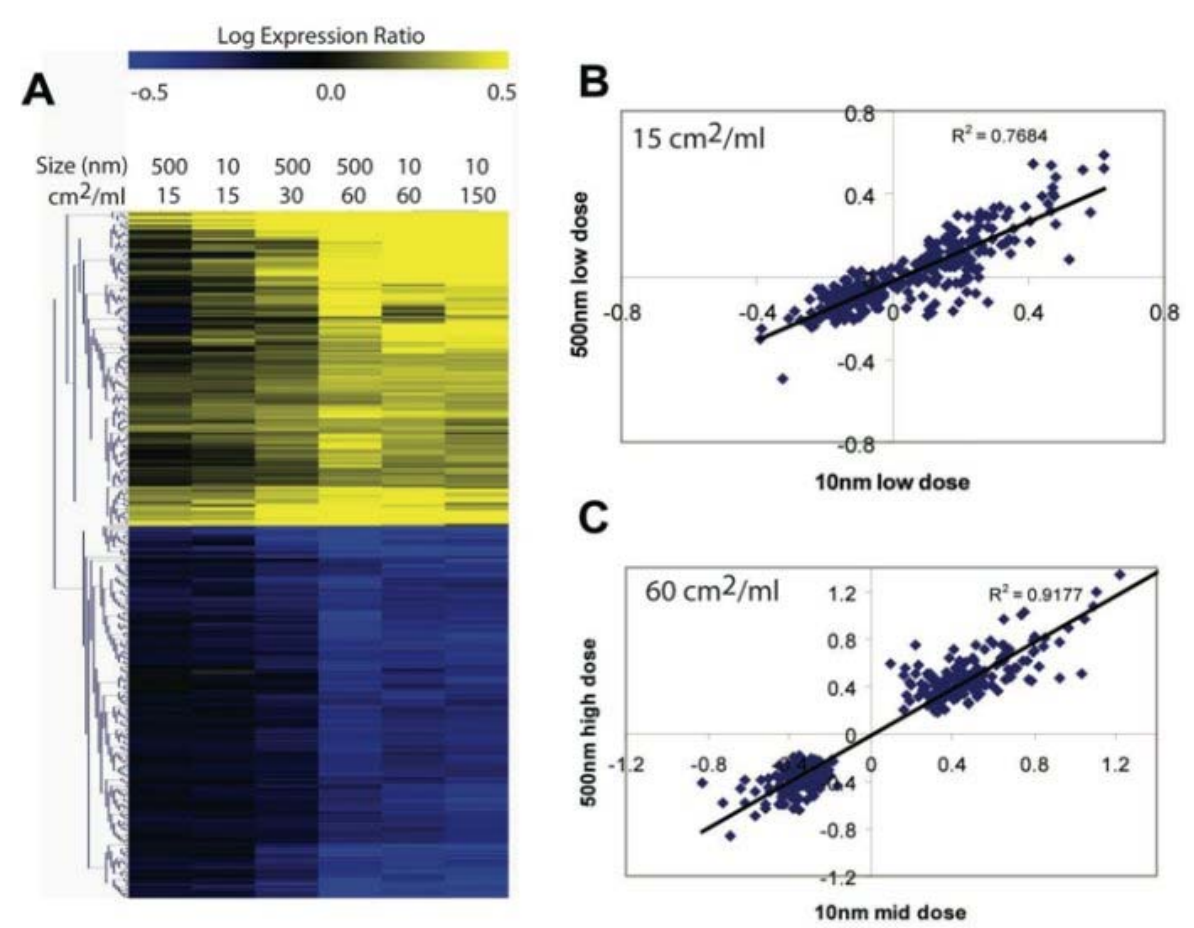

Figure 1. The relationship between $m R N A$ abundance changes and particle surface area dose for 404 genes regulated in response to 10- and 500-nanometer amorphous silica particles. Genes shown in the majority pattern A naturally clustered according to the particle surface area dose. In contrast, the genes for patterns $B$ and $C$ continued to separate by particle size and mass dose (data not shown). Correlation analysis for the majority pattern A genes indicated a strong overall correlation between the gene expression ratio and particle surface area dose across all treatment groups.

systems. Therefore, accurate interpretation of size-dependent biological responses to nanoparticles depends on the dose metric used for comparison - in this case, particle size. This is because for a given mass of particles, the total surface area increases with decreasing particle diameter, making it an essential determinant of the fraction of reactive groups on particle surfaces.

Using EMSL's particle characterization capability and transmission electron microscopy, this study demonstrated that the particle surface properties that dictate biocompatibility of amorphous silica are essentially the same at the nanoscale as a function of surface area. The generalized strategy described in this study can be extended to other nanomaterials both to identify mechanism of action and to prioritize more expensive toxicity studies in animals.

The team used a genome-wide approach to investigate whether the cellular pathways activated by amorphous silica are size dependent. Amorphous silica was chosen because of its increasingly important role in nanotechnology. It is used in production of high-efficiency photovoltaics and tires, and in consumer products such as drug delivery vehicles, toothpaste, sunscreen, cosmetics and nutraceuticals.

They performed whole genome microarray analysis of the early gene expression changes induced by 10 and 500-nm particles. These showed that the magnitude of change for the majority of genes affected 
correlated more tightly with particle surface area than either particle mass or number. The researchers also identified particle size-specific gene expression changes (Figure 1).

Using advanced bioinformatics approaches, the team was able to demonstrate that the biological pathways represented by the size-specific gene expression changes were nearly identical, irrespective of particle size. Direct comparison of the cell processes represented in the 10- and 500-nm particle gene sets using gene set enrichment analysis revealed that among 1009 total processes represented in the data, none were statistically enriched for one particle size group over the other.

The key mechanisms involved in silica nanoparticle-mediated gene regulation and cytotoxicity have yet to be established. For example, scientists don't know whether macrophage cell responses are initiated by particle contact with the cell membrane or if receptor binding or particle internalization is required. Such studies should help in developing strategies for enhancing the beneficial properties of nanomaterials while reducing potential adverse health effects mediated through macrophage interactions.

The research, sponsored by the National Institutes of Health and internal funding at PNNL, was published in Toxicological Sciences.

\title{
Citation
}

Waters KM, LM Masiello, RC Zangar, BJ Tarasevich, NJ Karin, RD Quesenberry, S Bandyopadhyay, JG Teeguarden, JG Pounds, and BD Thrall. 2009. "Macrophage Responses to Silica Nanoparticles are Highly Conserved Across Particle Sizes.” Toxicological Sciences 107(2):553-569.

\section{Electronic Effects on the Surface Potential at the Vapor-Liquid Interface of Water}

\author{
SM Kathmann, ${ }^{(a)}$ IFW Kuo, ${ }^{(b)}$ and CJ Munday ${ }^{(a)}$ \\ (a) Pacific Northwest National Laboratory, Richland, Washington \\ (b) Lawrence Livermore National Laboratory, Livermore, California
}

One of the toughest challenges in molecular simulation is to construct computational models that contain enough of the right physics to produce results scientists can trust. EMSL users from the Pacific Northwest National Laboratory and Lawrence Livermore National Laboratory have solved part of that problem. They have produced the first model to faithfully depict the electric field present at the point, or interface, where the liquid and vapor forms of water meet. The model also comes closer than other simulations to matching calculations derived from experiment.

In molecular simulation, a model is only as good as the physics that goes into it. More physics leads to better models. The effects of electrons and how to include them in models can make a big difference in the accuracy of a model. A research team from Pacific Northwest National Laboratory and Lawrence Livermore National Laboratory found that researchers have to consider the electrons individually if they want an accurate representation of an electric field.

A new model developed by the team provides an improved tool for acquiring that information (Figure 1). The model calculates the molecules' full electronic charge distribution-all of the water electrons and how they interact with other electrons. The type of information this model can provide is significant in 
many areas of chemical physics research, including energy, biology, materials science and global warming.

Scientists know that the dominant interaction among molecules is electrical and that very interesting chemistry occurs at interfaces, where one form of matter meets another. From electricity on a child's balloon that makes your hair stand up, to electron transport in transistors, electric fields are what make things move. Yet calibrating an electric field is immensely complex, even for a molecule as simple as water.

Surface potential is the term given to the measurement of an electric field at an interface. Scientists use surface potential calculations to describe and predict chemical reactions at a vapor-liquid interface. Many scientists have attempted to determine the surface potential of the vapor-liquid interface of water. But, they do not agree on the importance of the field or if the charge distribution is positive or negative.

The primary obstacle has been lack of computing power. The high-performance computing resources enabled the research team to apply quantum physics to the problem. These resources are located at EMSL and Lawrence Livermore National Laboratory.

The new model revealed that the strength of the electric field is much smaller than previously estimated and that the charge is always negative.

The team started by calculating the behavior of the electronic cloud that envelops every water molecule at the vapor-liquid interface. In water that is either all liquid or all gas, it is the intermingling of these electronic clouds that holds the molecules together. However, electrons behave differently where the liquid and vapor meet. This interface is a sort of no-man's-land, where electrons move from one state to the other and back again, aiding or restricting chemical processes and other molecular interactions.

The team then compared the new model with previous models and experiments. Earlier models viewed the electrons in water as set along certain pathways or gathered at the nuclei of the atoms. Dealing with the electrons as a cloud confirmed predictions that the density of the electrons significantly influences the electronic properties of the vapor-liquid interface of water. 
Future studies will seek to further quantify explicit inclusion of the electronic charge cloud. For example, researchers will address the surface potential and electric field at the interface between a salt crystal and liquid water, as well as electronic effects on the reaction path of various ions through the vapor-liquid interface of water.

This research was supported by the DOE Office of Basic Energy Sciences and was published in the Journal of the American Chemical Society.

\title{
Citation
}

Kathmann SM, IFW Kuo, and CJ Mundy. 2008 "Electronic Effects on the Surface Potential at the VaporLiquid Interface of Water." Journal of the American Chemical Society, 130:16556-16561.

\section{Self-Assembled $\mathrm{TiO}_{2}$-Graphene Hybrid Nanostructures for Enhanced Li-Ion Insertion}

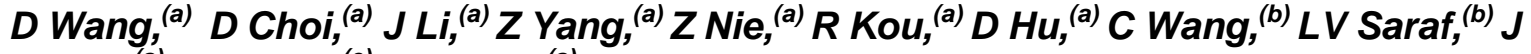 \\ Zhang, ${ }^{(a)}$ IA Aksay, ${ }^{(c)}$ and $\mathrm{J}$ Liu $^{(a)}$ \\ (a) Pacific Northwest National Laboratory, Richland, Washington \\ (b) EMSL, Richland, Washington \\ (c) Princeton University, Princeton, New Jersey
}

Many ideas to reduce the nation's oil addiction require an effective battery. For example, plug-in hybrid electrical vehicles need better batteries to drive longer distances. Solar farms only produce electricity when the sun shines. Rechargeable batteries are needed to capture electricity for use on cloudy days. Lithium ion batteries, used in camcorders and other devices, are a popular option, because pound for pound, they are some of the most energetic rechargeable batteries available. However, these batteries need to store more energy. New titanium oxide structures on carbon sheets devised by EMSL users and researchers could help the batteries hold more energy.

High capacity, safe batteries are needed for efficient hybrid or electrical vehicles and for storing and releasing electricity from intermittent power sources like wind turbines and solar panels. That's where an innovation by scientists at Pacific Northwest National Laboratory, EMSL, and Princeton University comes in. The researchers devised a method for building tiny titanium oxide and carbon structures that greatly improve the performance of lithium ion batteries. This new material stores twice as much electricity at high charge/discharge rates as batteries that don't use it.

Rechargeable lithium ion batteries, popular in cell phones, camcorders, and other devices, are based on the movement of a lithium ion - a lithium atom minus an electron. The lithium ion begins its journey attached to a metal cylinder or sheet, known as an electrode. The ion pushes off the electrode, moves through a liquid, and attaches itself to an electrode on the other side. The ion's movement generates electricity, powering the battery. The researchers' new material, titanium dioxide crystals attached to a thin carbon sheet called graphene, is incorporated into the battery's negative electrode. The carbon/titanium material greatly improves the ion's ability to move in the electrode to provide a high capacity at high charge/discharge rate. 
The challenge in designing this material was water. The researchers used water to reduce the cost of manufacturing. The precursors for the titanium dioxide crystals mixed well in water, easily dispersing. However, the graphene is hydrophobic or water fearing. Like oil or grease, it does not mix in water. The solution? The active ingredient in many types of detergents: sodium dodecyl sulfate. This long, chain-like molecule contains a cluster of chemicals, or a head at one end, that mixes well with water. It has a long tail that grabs hold of hydrophobic materials. So, adding sodium dodecyl sulfate allows the graphene to evenly mix in the water with the precursors for the oxide crystals.

The sodium dodecyl sulfate not only solves the hydrophobic/hydrophilic incompatibility problem, it also provides a molecular template for the crystals to form and grow. Using the template, the titanium oxides form tiny crystals on the graphene sheets.

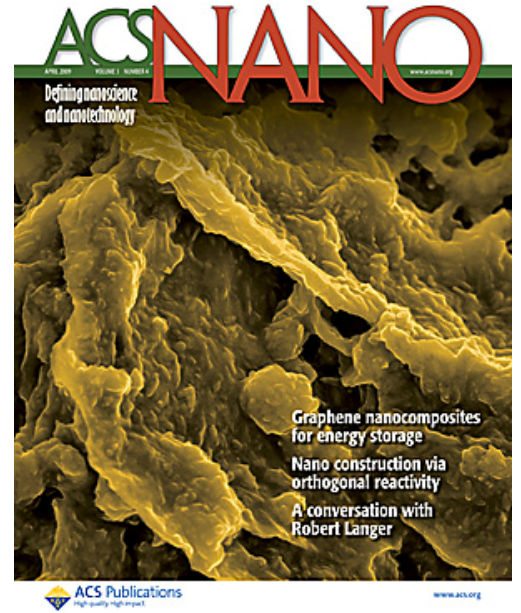

Figure 1. This research was featured on the April 2009 cover of ACS Nano.

The researchers studied the resulting materials using EMSL's transmission electron microscopy. The resulting images showed the desired titanium dioxide crystals formed on the graphene sheets. This process is being used by PNNL researchers in the Transformational Materials Science Initiative to precisely design other materials to further increase the capacity and stability of batteries.

This research was funded by Laboratory-Directed Research and Development Program at PNNL and by DOE's Office of Basic Energy Sciences. In addition support was given from the Defense Advanced Research Projects Agency and Army Research Office/Multidisciplinary University of Research Initiative. The research was featured on the cover of ACS Nano (Figure 1).

\title{
Citation
}

Wang D, D Choi, J Li, Z Yang, Z Nie, R Kou, D Hu, C Wang, LV Saraf, J Zhang, IA Aksay, and J Liu. 2009. "Self-Assembled $\mathrm{TiO}_{2}$-Graphene Hybrid Nanostructures for Enhanced Li-Ion Insertion." ACS Nano 3(4):907-914.

\section{Molecular Characterization of Nitrogen Containing Organic Compounds in Biomass Burning Aerosols Using High Resolution Mass Spectrometry}

\author{
A Laskin, ${ }^{(a)}$ JS Smith, ${ }^{(b)}$ and J Laskin ${ }^{(b)}$ \\ (a) EMSL, Richland, Washington \\ (b) Pacific Northwest National Laboratory, Richland, Washington
}

Every year, ponderosa wildfires occur along the nation's West Coast. While some fires are accidental, others are prescribed land management events. Using EMSL resources, researchers found that smoldering fires such as those in controlled burns produce more alkaloids than blazing fires. Because some plant alkaloids might be harmful, the result could affect planned fires upwind of human populations. 
Smoke from burning ponderosa pines contains previously undetected alkaloids, according to a study published by scientists working at EMSL (Figure 1). The alkaloids are potent mutagens that can affect human health and ecosystems in areas near or downwind of the fires. Researchers have long suspected the presence of alkaloids in smoke particles produced in forest fires, but no direct measurements had been made.

With the help of the Forest Service Fire Sciences Laboratory, a team from Pacific Northwest National Laboratory and EMSL sampled the smoke from smoldering fires of ponderosa pine and underbrush. Then, they devised a method that provides highly detailed information about the smoke's composition. This method included using EMSL's LTQ-OrbitrapTM high-resolution mass spectrometer to characterize the smoke. Compared to other studies, the team found that $70 \%$ of the molecules detected in the smoke had not been previously reported. More than $30 \%$ of newly detected species were alkaloids.

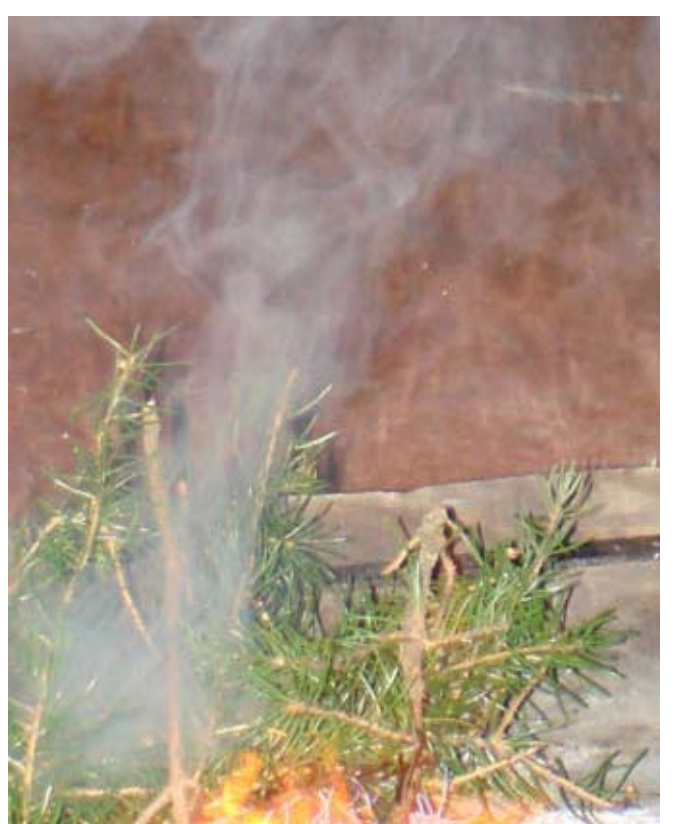

Figure 1. Previously undetected compounds were detected in smoke from a smoldering fire with a new method devised at EMSL.

This and earlier studies by the team will aid in understanding the possible effects of smoke particles emitted during forest fires on the environment and human health. The results suggest that smoke might carry biologically useful nitrogen in the form of alkaloids. In addition, alkaloids may present a considerable source of basic compounds in smoke particles, which can impact cloud formation processes important to agriculture and water supplies.

The DOE Office of Basic Energy Science and Office of Biological and Environmental Research as well as the Science Undergraduate Laboratory Internship program funded the research. Results of this research were published in Environmental Science and Technology and Analytical Chemistry.

\section{Citations}

Smith JS, A Laskin, and J Laskin. 2009. "Molecular Characterization of Biomass Burning Aerosols Using High Resolution Mass Spectrometry." Analytical Chemistry 81:1512-1521. doi: 10.1021/ac8020664

Laskin A, J Smith, and J Laskin. 2009. "Molecular Characterization of Nitrogen Containing Organic Compounds in Biomass Burning Aerosols Using High Resolution Mass Spectrometry." Environmental Science and Technology. doi: 10.1021/es803456n 


\title{
Symmetry-Driven Spontaneous Self-Assembly of Nanoscale Ceria Building Blocks to Fractal Superoctahedra
}

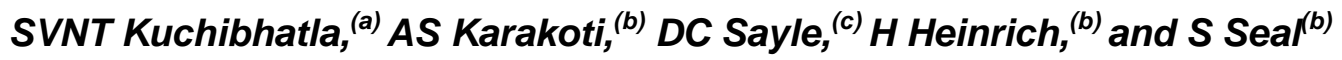 \\ (a) EMSL, Richland, Washington \\ (b) University of Central Florida, Orlando, Florida \\ (c) Defence Academy of the United Kingdom, Swindon, United Kingdom
}

The evolution of a self-assembled, oxide superoctahedral structure has been elucidated for the first time, and this unique finding was featured on the cover of Crystal Growth and Design-a journal ranked first in the field based on impact factor. Through this research, the ability to understand and tailor nanomaterials with better properties can lead to sustainable, greener energy prospects; environmental protection; and the detection, treatment, and prevention of diseases using nanomedicine.

Researchers at EMSL and their collaborators from the University of Central Florida and the Defence Academy of the United Kingdom used a combination of experimental tools at UCF and EMSL as well as theoretical tools at the Cambridge-Cranfield HPC facility to study cerium oxide. Because cerium oxide is crucial for solid oxide fuel cells, solar cells, catalysis, and biomedicine, understanding its nanostructures is vital to improve their performance in those applications.

To characterize the superstructures, the research team applied high-resolution transmission electron microscopy and fastFourier transform analysis. HRTEM yielded structural and morphological information (Figure 1). FFT analysis allowed the researchers to study a specific sample area of interest such that crystallographic information from even single nanoparticles could be derived. Integrating the team's experimental data with atomistic modeling revealed a stepwise process for superstructure formation. Importantly, the process occurred naturally - at
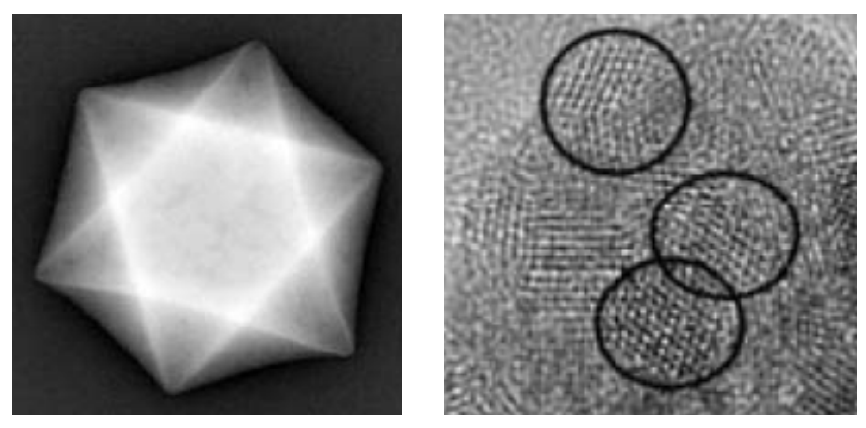

Figure 1. Ceria oxide forms octohedral superstructures (TEM image at left) composed of octahedral nanoparticles (HRTEM image at right; circles indicate individual nanoparticles). room temperature and pressure and without surfactants. First, cerium oxide molecules form individual octahedral nanoparticles that are shaped like two pyramids stuck together at their bases. This step was successfully predicted by molecular dynamics simulations, and that prediction was confirmed experimentally. Next, the octahedra stack tightly together via fractal self-assembly to yield superoctahedral structures. Understanding the orientation of the interfaces between two adjoining octahedral nanoparticles is particularly important. These interfaces may have a low or high energy conformation - in other words, the surfaces of the nanoparticles may lie against one another more or less comfortably. A high-energy interface is prone to defects that may act as potential reaction sites, making the defects favorable for improved performance.

Understanding the factors that control oxide nanostructure size, shape, and self-assembly enable the design of new materials with improved properties for energy, catalytic, or biomedical applications. The types of information obtained in these studies demonstrate the importance of EMSL's capability 
development to enable the design and growth of increasingly complex materials and their characterization with unprecedented (atomic level) resolution.

This work was supported by the National Science Foundation and the Cambridge-Cranfield HPC facility.

\title{
Citation
}

Kuchibhatla SVNT, AS Karakoti, DC Sayle, H Heinrich, and S Seal. 2009. "Symmetry-Driven Spontaneous Self-Assembly of Nanoscale Ceria Building Blocks to Fractal Superoctahedra." Crystal Growth \& Design 9(3)1614-1620. DOE:10.1021/cg801358z

\section{Clay Nanoparticles-Supported Single-Molecule Fluorescence Spectroelectrochemistry}

\author{
C Lei, ${ }^{(a)} \mathrm{D} \mathrm{Hu},{ }^{(a)}$ and EJ Ackerman ${ }^{(a)}$ \\ (a) Pacific Northwest National Laboratory, Richland, Washington
}

Scientists at Pacific Northwest National Laboratory devised a method to hold certain types of molecules still and measure if they blink; that is, gain or lose electrons. Mother Nature is very good at making catalysts: proteins that quickly add and remove electrons to create the desired products. Scientists would like to design molecules that mimic Mother Nature's catalysts, but do not yet know how. This research provides an important tool to learn if, when and why single molecules transfer electrons. By understanding electron transfers, scientists can gain the insights needed to design, atom by atom, catalysts that effectively turn water into hydrogen fuel or other reactions.

The scientists combined two existing methods to study immobilized individual molecules of the dye cresyl violet. The first technique is electrochemistry, which allows scientists to add and remove electrons to molecules at specific intervals. The second method is to use a single-molecule fluorescence microscope to record the light emission of the molecules. It uses a dye that gives off light under specific conditions. For example, when cresyl violet loses an electron, it gives off a red glow. When it gains an electron, it goes dark.

So, the researchers built an electrochemical cell that can measure electron movement on a type of conductive glass surface. Then, they placed a few drops of a clay solution on the glass. The liquid dried onto the glass, forming a transparent clay film. Then, they added cresyl violet. The dye attached to the clay film, holding the dye in place. This left the scientists with a piece of glass with a few dye molecules securely attached to it.

Next, they added to and removed electrons from the molecules at regular intervals by changing the electrochemical voltages. Simultaneously, they recorded the light flashes using a single-molecule fluorescence microscope at EMSL.

The method worked well, providing information about single-molecule effectiveness; that is, single molecules regularly gained and lost electrons while the molecules underwent cycles of blinking, dark and then bright. 
The researchers plan to use this method to interrogate other molecules and gain the knowledge necessary to design catalysts that mimic Mother Nature's most effective molecules.

DOE's Office of Basic Energy Sciences funded this research, which was featured in Nano Letters.

\title{
Citation
}

Lei C, D Hu, and EJ Ackerman. 2009. "Clay Nanoparticles-Supported Single-Molecule Fluorescence Spectroelectrochemistry.” Nano Letters 9(2):655-658.

\section{Line Intensities for the $\mathrm{v}_{1}, \mathrm{v}_{3}$, and $\mathrm{v}_{1}+\mathrm{v}_{3}$ Bands of ${ }^{34} \mathrm{SO}_{2}$}

\author{
JM Flaud, ${ }^{(a)}$ WJ Lafferty, ${ }^{(b)}$ and RL Sams ${ }^{(c)}$ \\ (a) Laboratoire Inter-Universitaire des Systemes Atmospheriques, Paris, France \\ (b) National Institute for Standards and Technology, Gaithersburg, Maryland \\ (c) Pacific Northwest National Laboratory, Richland, Washington
}

Acid rain and respiratory problems are related to sulfur dioxide emissions from coal-fired power plants, volcanic eruptions, and other sources. To design cost-effective sulfur dioxide remediation tools and to effectively regulate emissions, scientists must be able to monitor sulfur dioxide at specific sources and in the atmosphere. The infrared crosssection values of ${ }^{34} \mathrm{~S}_{16 \mathrm{O}_{2}}$ determined at EMSL allow field measurements to be done with greater accuracy and source discernment.

Using EMSL resources, an international team completed a detailed infrared spectral analysis of ${ }^{34} \mathrm{~S}^{16} \mathrm{O}_{2}$. This analysis of this lesser studied sulfur isotope allows researchers to more accurately quantify the total amount of sulfur dioxide in the atmosphere. Measuring the ratio ${ }^{34} \mathrm{SO}_{2}$. and ${ }^{32} \mathrm{SO}_{2}$ may provide insights into discerning between specific natural and human-made sources of sulfur dioxide that contributes to, for example, acid rain (Figure 1). With infrared spectroscopy, atmospheric sensing can be done over meters to many kilometers, from the ground, a balloon, aircraft, or a satellite.

The team began by studying the isotopic sample using EMSL's high-resolution Fourier transform infrared spectrometer. After a preliminary analysis, additional analysis was done at the National Institute for Standards and Technology. The analysis revealed new information about the structure of the molecule, the rotational and

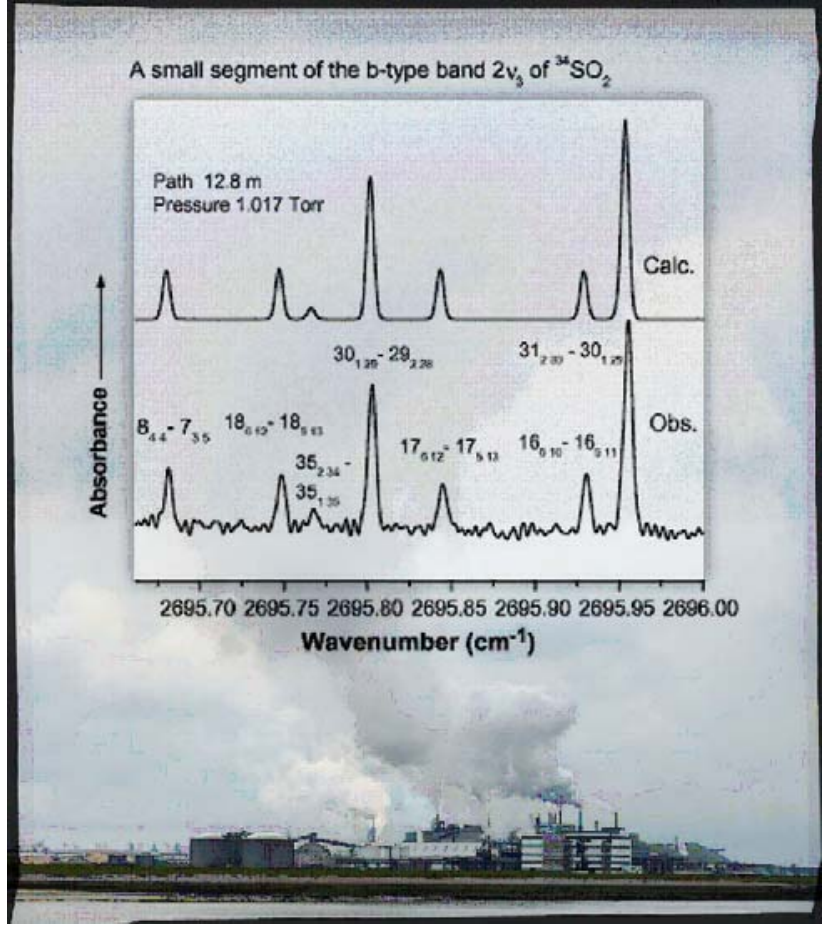

Figure 1. EMSL users obtained detailed data on the industrial pollutant $34 \mathrm{SO}_{2}$. vibrational energies of the molecule, and the interactions of these energies within the molecule. This work also measured, for the first time, the infrared absorption cross-sections for individual rotationalvibrational transitions of ${ }^{34} \mathrm{~S}^{16} \mathrm{O}_{2}$., vital for eco-monitoring. 
This study provides a more accurate experimental view of the structure, bonding, and vibrational and rotational energy patterns of ${ }^{34} \mathrm{~S}^{16} \mathrm{O}_{2}$. The results provide detailed information about the potential energy surface of a molecule with a modest number of electrons. The National Aeronautics and Space Administration's Upper Atmosphere Research Program; DOE's Office of Basic Energy Sciences; and DOE's EMSL Operations funded this research. It was featured in the Journal of Quantitative Spectroscopy and Radiative Transfer

\section{Citation}

Lafferty W, JM Flaud, and RL Sams. 2009. "Line intensities for the $v 1, v 3$, and $v 1+v 3$ Bands of ${ }^{34} \mathrm{SO}_{2}$." Journal of Quantitative Spectroscopy and Radiative Transfer 110:669-674.

\section{Co-Ordinatively Unsaturated $\mathrm{Al}^{3+}$ Centers as Binding Sites for Active Catalyst Phases on $\mathrm{Y}-\mathrm{Al}_{2} \mathrm{O}_{3}$}

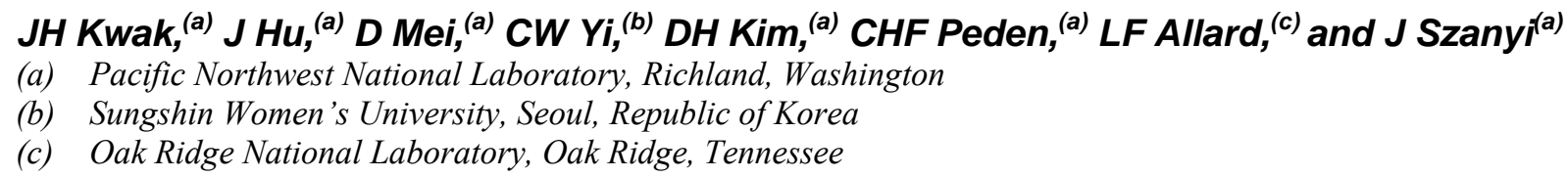

Catalysts convert useless or unwanted chemicals into useful or more desirable ones. Research reported in Science reveals new, important details about a common catalyst: how rafts of chemically reactive platinum form in the catalyst. The new work yields insights into how to improve the industrial catalyst for oil refining, chemicals processing and environmental uses.

The study shows that aluminum atoms in the supporting material thirsty for another bond grab and anchor platinum (Figure 1). The anchors allow platinum atoms to group into rafts that float above the supporting surface, providing ample space for catalytic reactions.

EMSL users in the Institute for Interfacial Catalysis at Pacific Northwest National Laboratory and at Oak Ridge National Laboratory performed the analysis of

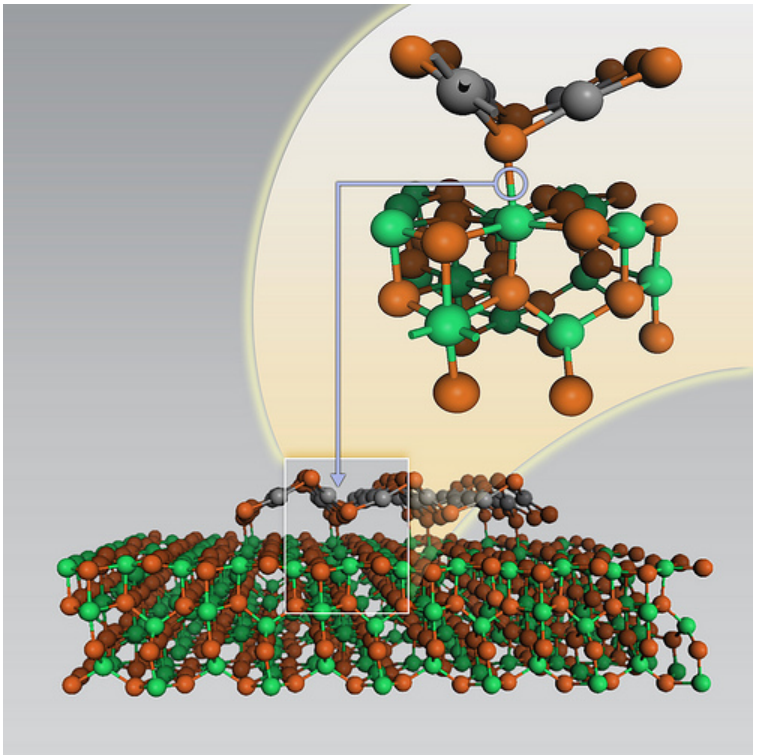

Figure 1. Rafts of catalytic platinum oxide float above a sea of aluminum oxide, anchored by bonds between platinum and aluminum. the industrial catalyst known as aluminum oxide-supported platinum. Such precious metal and oxide combinations are the most common kinds of industrial catalysts. The new work will help engineers control the preparation of the catalyst, which will lead to performance improvements.

The research team has been able to specifically identify an important site for the anchoring of platinum on the aluminum oxide surface that's formed during synthesis. Although platinum rafts have been observed before, this is the first time the team has had a clear molecular-level view of the processes that create them. 
In these catalysts, the oxides are merely a surface on which the precious metals sit while the metals break and form bonds in other molecules, such as those found in automobile exhaust. The most efficient catalysts spread the precious metals evenly over the surface of the oxide. Inefficient ones have precious metal atoms balled up in clumps, with the interior atoms unavailable to do their job on incoming molecules. Chemists working with gamma-aluminum oxide supports and platinum metal knew that under some conditions, rafts of platinum atoms could form on the oxide surface. Unlike balls of atoms, rafts present most of their resident atoms to incoming molecules, making them desirable structures. But to control production of the rafts, the team had to learn how they formed.

To find out, the team used powerful instruments at EMSL and the High Temperature Materials Laboratory at ORNL. The team prepared gamma-aluminum oxide under typical catalyst synthesis conditions and examined the supporting material before and after adding platinum.

First, they examined the chemical nature of the support using EMSL's 900-megahertz NMR spectrometer. The NMR provided unprecedented resolution of the aluminum oxide support, which allowed the team to identify aluminum atoms with certain properties. By their chemical nature, aluminum atoms prefer to be bound to either four or six atoms. The team found, in the absence of platinum, that some were bound to an uncomfortable five. Adding platinum to the mix, however, caused the number of aluminum atoms with five bonds to decrease, and the number of atoms with six bonds to increase. The number of four-bonded atoms stayed constant, suggesting that platinum atoms anchor at sites with comehither, five-bonded aluminum atoms, so-called penta sites. The team found they could increase the number of penta sites by raising the temperature during catalyst synthesis. More penta sites meant more platinum atoms bound to the support.

Having found anchor points, the team zoomed in with the JEOL 2200FS aberration-corrected microscope, which could discern individual platinum atoms, at HTML. At low concentrations of the metal, individual platinum atoms showed up as bright spots scattered across the dark surface. At higher concentrations, the telltale image of platinum rafts could be seen above the aluminum oxide.

Lastly, the team showed that penta-aluminum atoms were needed for the rafts to form. Alpha-aluminum oxide does not contain penta sites. When the researchers looked under the microscope at catalyst material formed with alpha-aluminum oxide, platinum atoms formed balls that tottered around on the surface instead of tidy rafts. Theoretical analysis that took into account all the experimental data yielded a model of how the catalyst material forms. The results provide insight into how they could produce a better performing catalyst.

Finding the conditions that will allow the chemists to control the number and distribution of penta sites will be the subject of future research. This work was supported by DOE's Basic Energy Sciences and Energy Efficiency and Renewable Energy.

\section{Citation:}

Kwak JH, J Hu, D Mei, CW Yi, DH Kim, CHF Peden, LF Allard, and J Szanyi. 2009. "Co-Ordinatively Unsaturated $\mathrm{Al}^{3+}$ Centers as Binding Sites for Active Catalyst Phases on $\gamma-\mathrm{Al}_{2} \mathrm{O}_{3}$." Science DOI 10.1126/science.1176745. 


\title{
Observation of a Remarkable Temperature Effect in the Hydrogen Bonding Structure and Dynamics of the $\mathrm{CN}^{-}\left(\mathrm{H}_{2} \mathrm{O}\right)$ Cluster
}

\author{
XB Wang, ${ }^{(a)(b)}$ JC Werhahn, ${ }^{(c)}$ LS Wang, ${ }^{(a)(b)} K$ Kowalski, ${ }^{(d)}$ A Laubereau, ${ }^{(c)}$ and \\ SS Xantheas ${ }^{(a)}$ \\ (a) Washington State University Tri-Cities, Richland, Washingon \\ (b) Pacific Northwest National Laboratory, Richland, Washington \\ (c) Technische Universitat Munchen, Garching, Germany \\ (d) EMSL, Richland, Washington
}

Scientists have long known that molecules dance about as the temperature rises, but now researchers know the exact steps that water takes with a certain molecule. Results with small, electrically charged cyanide ions and water molecules reveal that water zips around ions to a greater extent than expected. The findings, using EMSL computational and experimental resources, improve understanding of a chemical interaction important in atmospheric sciences.

A team comprised of researchers from Washington State University, Pacific Northwest National Laboratory, Technische Universitatet Munchen, and EMSL have developed a new instrument to probe the dynamics of ions in water. Combining theory and modeling to make sense of those experiments have given the team a deeper fundamental understanding of what is happening with water. The results were published on the cover of the September 3, 2009, issue of Journal of Physical Chemistry A.

Atmospheric scientists want to know how small particles flutter through water vapor in the sky. To get at the basics, they study a simpler interaction: water and ions, small atoms or molecules that have a slight electrical charge and exist everywhere in nature. For example, when common table salt — sodium chloride - dissolves in water, the negatively charged chloride ions $\left(\mathrm{Cl}^{-}\right)$and the positively charged sodium ions $\left(\mathrm{Na}^{+}\right)$each interact separately with the water molecules.

Previous work with chloride ions and water has yielded conflicting results about how a water molecule (which is shaped like a boomerang) and a chloride ion (shaped like a ball) face each other. Other groups study barbell-shaped cyanide ions because many molecules found naturally in the environment contain cyanide. The chemical interactions of water and either chloride or cyanide are influenced by the charge and the shape of the molecules, as well as the temperature in which they find themselves.

But directly observing temperature's role in how water and cyanide ions interact has been difficult. So, the team developed a unique instrument that allowed them to precisely control the temperature down to almost absolute zero, or the temperature at which everything freezes. The team used temperaturecontrolled photoelectron spectroscopy in EMSL to determine how tightly one cyanide ion and one to three water molecules interact at the very low temperature of $-438^{\circ} \mathrm{F}(12 \mathrm{Kelvin})$ and again at ambient temperature of $80^{\circ} \mathrm{F}$ (equivalent to 300 Kelvin).

The team measured the molecules' electron binding energy at low and high temperatures. This energy is an indication of how tightly the molecules hold onto their electrons - the tighter the hold, the stronger the bonds that will form between molecules. The team found that ones at low temperature exhibited higher electron binding energy than the ones at high temperatures, as they had expected. However, the difference between the two scenarios was greater than the team expected. 
To explore the unexpected difference in energy, the researchers ran computer simulations on the Chinook supercomputer in EMSL. This also let them determine how the boomerang-shaped water and barbellshaped cyanide faced each other. First they estimated how much energy the molecules used to take different configurations. Then they compared the computer-based estimates to the data they collected in their unique instrument at different temperatures. The team found that the molecules behaved differently at cold and warm temperatures. At lower temperatures, the boomerang-shaped water held still while the cyanide teetered at the end of one of water's two arms. There, the cyanide flipped, sometimes pointing its carbon $(\mathrm{C})$ atom towards the water's arm, and sometimes pointing its nitrogen $(\mathrm{N})$. At the coldest temperature tested, $-438^{\circ} \mathrm{F}$, the molecules froze, with cyanide pointing its nitrogen end at the water.

At ambient temperatures, however, the barbell-shaped cyanide held steady while the water molecule rocked and flipped around the cyanide. Although the researchers were surprised at how much the water moved, the many positions water could take explained why they saw less electron binding energy than they expected at room temperature: A wiggly water means that the bond between molecules isn't that tight.

The results also explain the conflicting results with chloride ions and water, because of the importance of temperature on that interaction as well.

The researchers plan to follow up with studies that include many water molecules and ions at once, as well as with more complex ions than cyanide. The research was funded by DOE's Office of Basic Energy Sciences.

\section{Citation:}

Wang XB, JC Werhahn, LS Wang, K Kowalski, A Laubereau, and SS Xantheas. 2009. "Observation of a Remarkable Temperature Effect in the Hydrogen Bonding Structure and Dynamics of the $\mathrm{CN}^{-}\left(\mathrm{H}_{2} \mathrm{O}\right)$ Cluster.“ Journal of Physical Chemistry A, DOI 10.1021/jp9034002.

\section{Single Wall Diesel Particulate Filter (DPF) Filtration Efficiency Studies using Laboratory-Generated Particles}

\section{J Yang, ${ }^{(a)}$ M Stewart, $^{(a)}$ G Maupin, ${ }^{(a)}$ D Herling, ${ }^{(a)}$ and A Zelenyuk ${ }^{(a)}$}

(a) Pacific Northwest National Laboratory, Richland, Washington

Studies of how diesel particulate filters (DPFs) trap particles use a fundamental scientific approach to understand issues related to clean energy production and use. Such research has future potential to help manufacturers build better filters, a critical step toward tackling diesel exhaust emissions, leading to less air pollution.

A team of researchers from the Pacific Northwest National Laboratory used integrated computational and experimental resources at EMSL to investigate the ability of two types of diesel particulate filterssilicon carbide and cordierite - to trap small particles that diesel engines emit into our air (Figure 1). Consumers are enamored with the higher fuel efficiency diesel engines afford, but are put off by the higher exhaust particulate matter, or soot, diesel produces. Presently, DPFs are the most efficient means to trap the soot. EMSL researchers conducted a direct comparison between silicon carbide and cordierite DPFs as well as between experimental data and existing models that aim to predict DPF filtration 
efficiency and pressure drop. Nearly spherical laboratory-generated ammonium sulfate particles were used as soot surrogate in this study; they are ideally suited for comparing experimental results to computational simulations that treat soot agglomerates as spherical. To what extent these simplifications impact the accuracy of DPF simulations remains an open question.

The study theoretically modeled and experimentally tested the two ways in which filters block particulate matter. First, particles are captured in the filter holes; second, longer exposure causes the particles to form

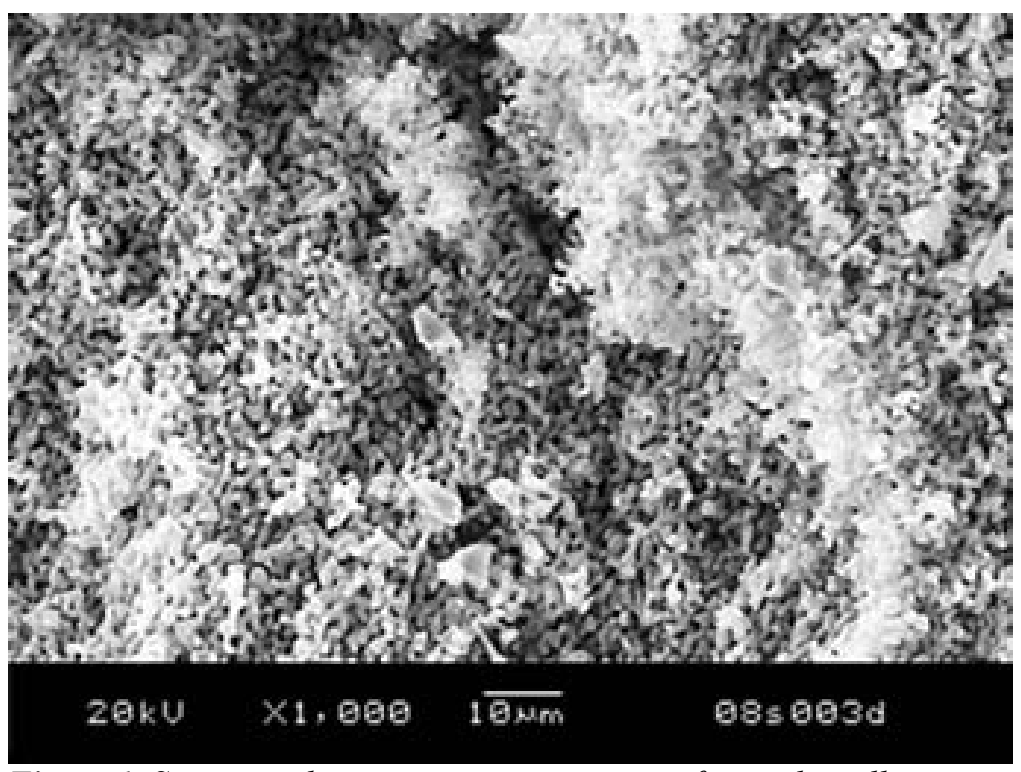

Figure 1. Scanning electron microscopy image of a single wall cordierite filter that was loaded with ammonium sulfate particles for 18 hours. The particles trapped on top of the filter form a thick cake. a dense "soot cake" on which particles are efficiently trapped. The researchers showed that the transition from filter capture "soot cake" can be identified by recording the change in pressure across the filters as a function of particle loading and that the filters trap small and large particles better than particles that are $\sim 80$ to $200 \mathrm{~nm}$ in aerodynamic diameter. Comparing experimental data and a simulation showed that the models overpredict the filter's trapping efficiency, and as a result they also predict shallower penetration than that observed in the experiment. The team will travel to Wisconsin to collaborate with General Motors and the University of Wisconsin researchers to investigate the behavior of DPFs when exposed to laboratory-generated soot particles and particles emitted by diesel engines under various operating conditions. The collaborators plan to characterize the physical and chemical properties of particle matters using EMSL’s Single-Particle Laser Ablation Mass Spectrometer, or SPLAT II.

This work, supported by the DOE Office of Energy Efficiency and Renewable Energy, was featured in Chemical Engineering Science.

\section{Citation:}

Yang J, M Stewart, GD Maupin, DR Herling, and A Zelenyuk. 2009. "Single wall diesel particulate filter (DPF) filtration efficiency studies using laboratory-generated particles." Chemical Engineering Science 64(8):1625-1634. 


\title{
No Confinement Needed: Observation of a Metastable Hydrophobic Wetting Two-Layer Ice on Graphene
}

\author{
GA Kimmel, J Matthiesen, M Baer, CJ Mundy, NG Petrick, RS Smith, Z Dohnalek, and \\ BD Kay \\ (a) Pacific Northwest National Laboratory, Richland, Washington \\ (b) Ruhr-Universitat Bochum, Bochum, Germany
}

For the first time, scientists have built completely flat, two-layer ice. While theoreticians have predicted that such ices are formed by squeezing water molecules between two surfaces, EMSL users from Pacific Northwest National Laboratory and Ruhr-Universitat Bochum are the first to create it. All it took was collaboration, creativity, and the absence of pressure. Creating this type of ice shows the structure of water on a water-fearing, or hydrophobic, surface. The structure of water at these surfaces is vital to understanding protein folding, and the assembly of cellular membranes and intracellular compartments.

Close collaboration between theoreticians and experimentalists from Pacific Northwest National Laboratory and Ruhr-Universitat Bochum, along with well-equipped laboratories and a supercomputer, allowed the team to build a layer of ice two molecules thick and then to determine how the atoms and electrons in that ice arranged themselves.

The experimentalists began with a thin sheet of carbon, known as graphene on top of a layer of platinum. Then, they introduced a small amount of water onto the surface in ultrahigh vacuum (that is, no pressure) and very low temperatures. While ice traditionally forms at 273 Kelvin, for this experiment, the temperature was dropped to $125 \mathrm{~K}$, about the temperature of an evening on the moon.

Next, the team used low-energy diffraction equipment that sends waves of slow-moving electrons at the surface. How those electrons bounce off the surface tells researchers a lot about the structure of the material.

The researchers found a layer of smooth ice had grown on the graphene, not the usual puckered layers of ice seen on water friendly or hydrophilic surfaces. In the new ice, the angles between the atoms in the water molecules were stretched or compressed compared to normal ice. So, the team subjected the ice to infrared spectroscopy, giving them information on how the water molecules were vibrating in the ice film. These results confirmed that the water molecules were in unusual configurations.

With these results, the theoreticians did complex calculations that determined the position of each of the atoms in the ice layer. The calculations were done with the software NW-ICE on a supercomputer at EMSL (Figure 1). The theoreticians found that in each layer of the ice, the water molecules formed slightly larger rings than normal. These six-sided
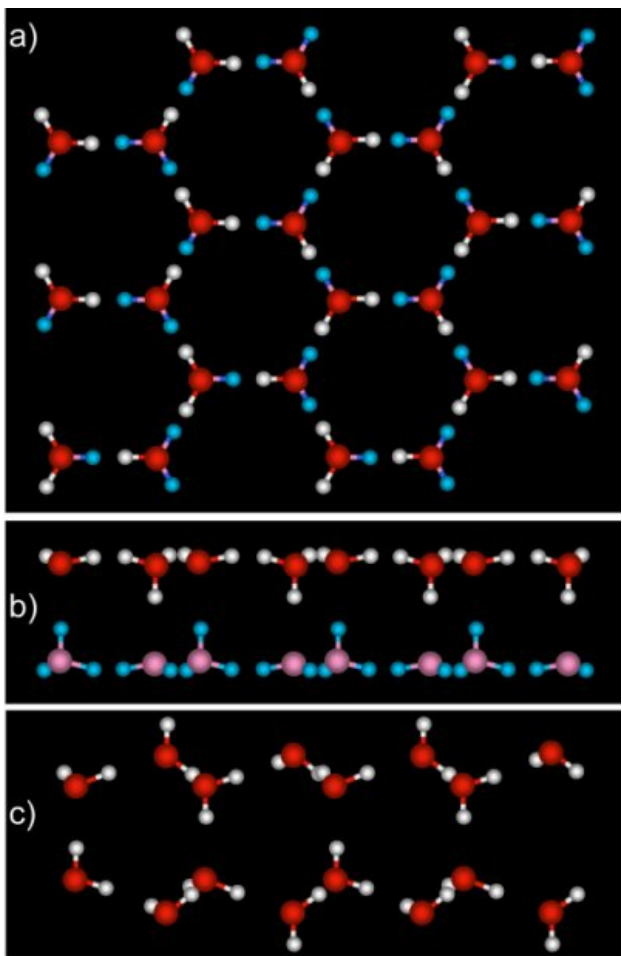

Figure 1. (a) Top view. The two-layer ice has hexagonal symmetry. (b) Side view. Two flat layers of molecules with hydrogen bonds connecting the layers. (c) Side view of normal, puckered hexagonal ice. 
rings stacked on top of each other, when looking down through the layers. They also found that each water molecule formed four hydrogen bonds - three with other molecules in the same layer, and one with water in the other layer. They also found that there are two types of water molecules: One type is parallel to the surfaces of the ice and its $\mathrm{H}-\mathrm{O}-\mathrm{H}$ angle is larger than normal. The other type connects the two layers and its $\mathrm{H}-\mathrm{O}-\mathrm{H}$ angle is smaller than normal.

The team, again integrating experiments and theory, are studying the temperature range over which the two-layer ice is stable and what happens when more water is added on top of the two-layer ice.

The research was supported by DOE's Office of Basic Energy Sciences and partially by Deutsche Forschungsgemeinschaft and by Fonds der Chemischen Industrie. The research was featured in Journal of the American Chemical Society.

\title{
Citation:
}

Kimmel GA, J Matthiesen, M Baer, CJ Mundy, NG Petrick, RS Smith, Z Dohnalek, and BD Kay. 2009. "No Confinement Needed: Observation of a Metastable Hydrophobic Wetting Two-Layer Ice on Graphene." Journal of the American Chemical Society 131(35):12838.

\section{Defect-Enhanced Charge Transfer by lon-Solid Interactions in SiC using Large-Scale $\boldsymbol{A b}$ Initio Molecular Dynamics Simulations}

\author{
F Gao, ${ }^{(a)} \mathrm{HXiao},{ }^{(b)} X \mathrm{Zu},{ }^{(b)} M$ Posselt, ${ }^{(c)}$ and WJ Weber ${ }^{(a)}$ \\ (a) Pacific Northwest National Laboratory, Richland, Washington \\ (b) University of Electron Science and Technology of China, Chengdu, China \\ (c) Forschungszentrum Dresden-Rossendorf, Institute of Ion Beam Physics and Materials Research, Dresden, \\ Germany
}

Radiation can come in a seemingly benign form like sunlight or in a more destructive form to materials, as in nuclear reactors and outer space. Either way, radiation interacts with the materials it touches. Understanding how those interactions damage or affect the properties of the material has challenged traditional computer modeling and simulation approaches. Now a team of EMSL users has shown that how electrons respond to radiation in a solid can alter the way permanent damage is created in the material and radically affect its mechanical, magnetic, electrical, and optical properties. Understanding how defects are formed helps researchers understand how material is changed by radiation, which is important for safe nuclear power and space exploration

Hard materials such as the steel used in nuclear reactors or natural minerals such as diamond look stable, but radiation exposure kicks the material's atoms off their highly organized lattices as ions that move freely inside the lattice. Previous studies led scientists to believe that the ions smashed into each other like cars in a demolition derby, with each ion car being a hard particle with immovable electrons embedded in the car's surface.

The team of EMSL users from Pacific Northwest National Laboratory, University of Electron Science and Technology of China, and Forschungszentrum Dresden-Rossendorf is finding that simply isn't the case. Instead of being embedded in the ion cars, the electrons sit like a cloud of exhaust around each ion, and they can actually leap from ion to ion and back again. The way they leap affects how the ions react to 
each other. Ions may move farther apart or closer, speed up or slow down. The researchers need to understand at a fundamental level how energy is transported by both electrons and ions. They used much higher-level theoretical calculations to look deeper than the level of the atom to understand the response and role of the electrons.

In a relatively new approach, the scientists used a single blast of low-level radiation to create damage, or atomic defects, in a silicon-based material so they could study the movement of both the electrons and silicon ions. Using supercomputers at EMSL and the National Energy Research Supercomputing Center, the researchers found that electrons jumped back and forth from the moving silicon ion to surrounding atoms (Figure 1). This movement ultimately created permanent defects, or damage, in the material. The researchers also found that electrons can be trapped by the defects created.

The work is the first time such intensive calculations could be

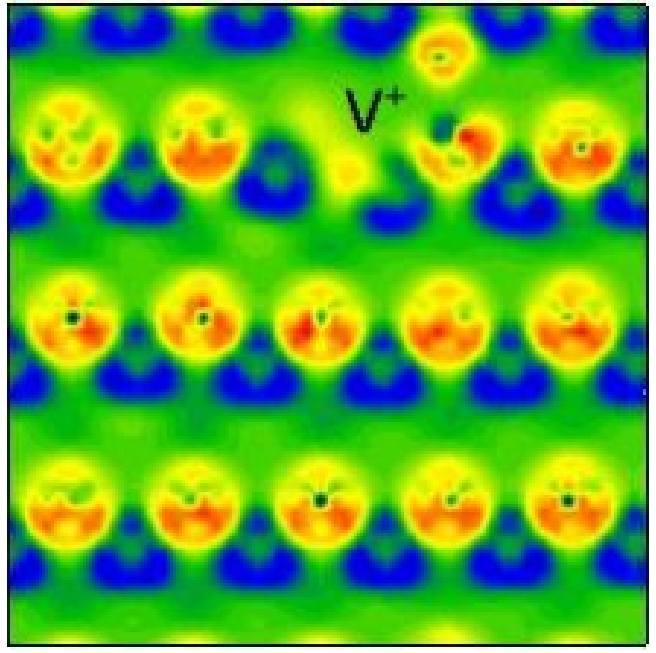

Figure 1. Scientists were surprised to find that electrons jump between ions when material is exposed to low levels of radiation, and how the charged vacancy, shown in yellow and indicated by $V+$, is formed. performed. The team modified state-of-the-art software and took advantage of recent advances in computer hardware to perform the calculations. They now hope to apply the work to more complex materials to look at how trapped electrons affect the way defects move and interact with each other when the material is exposed to more than one blast of radiation over longer times in much larger systems and at higher levels of radiation. Improved understanding and modeling of how electrons and ions respond to radiation will lead to improved materials and performance in safely managing nuclear fuels and waste forms.

This work was supported by the DOE Office of Basic Energy Sciences; the Department of Applied Physics, University of Electronic Science and Technology of China; and the Forschungszentrum DresdenRossendorf, Institute of Ion Beam Physics and Materials Research in Dresden, Germany.

\section{Citation:}

Gao F, H Xiao, X Zu, M Posselt, and WJ Weber. July 2009. "Defect-Enhanced Charge Transfer by IonSolid Interactions in SiC using Large-Scale Ab Initio Molecular Dynamics Simulations." Physical Review Letters 103(2):02745. DOI: 10.1103/PhysRevLett.103.027405 


\title{
On the Phase Diagram of Water with Density Functional Theory Potentials: The Melting Temperature of Ice $I_{h}$ with the Perdew-Burke- Ernzerhof and Becke-Lee-Yang-Parr Functionals
}

\author{
$S$ Yoo, ${ }^{(a)} X C$ Zeng, $^{(b)}$ and SS Xantheas ${ }^{(a)}$ \\ (a) Pacific Northwest National Laboratory, Richland, Washington \\ (b) University of Nebraska, Lincoln, Nebraska
}

Two popular computer models used by chemists and physicists to describe water do not correctly predict that ice melts at 32 degrees Fahrenheit, according to a study by EMSL users from Pacific Northwest National Laboratory and the University of Nebraska, Lincoln, that was compiled with the help of EMSL computing resources. More important, the study suggests that simulations of liquid water at room temperature with those models actually describe a supercooled glassy state, not the regular liquid. The study in the May 2009 Journal of Chemical Physics was the \#3 download paper in June.

The team pointed out the problem in two of the most popular DFT, or density functional theory, models in predicting the correct phase diagram of water. A DFT model describes the underlying interactions between water at the molecular level, incorporating the interactions between the nuclei and the electrons of the system. Both of those DFT models suggest that the melting temperature of ice is way too high, almost $150^{\circ} \mathrm{F}$ higher than the value that nature has settled on. So if you use those models to describe water at room temperature, you do not get the regular liquid but instead a supercooled glassy state that does not look like nature's most ubiquitous solvents. This finding will help researchers fine tune DFT models to achieve a closer match to what's observed during experiments.

Whether studying clouds, cancer, or catalysts, the behavior of water is the key factor. Clouds form from water and act as a testbed for a variety of processes that ultimately affect earth's climate. Cancer treatments must work with the water found in cells. And catalysts both speed reactions in water and use the liquid as a feedstock to create renewable energy sources such as hydrogen.

Scientists in all of these fields need to understand, at the molecular level, how water behaves under different conditions and in different environments. For example, how does the behavior of water molecules and sulfur oxide impact the creation of acid rain? The DFT models are used to understand how changes at the atomic and molecular levels influence behaviors we can see and touch. The more realistic these models are, the easier time scientists have understanding and controlling reactions.

The research, which was supported by DOE's Office of Basic Energy Sciences and the Nebraska Research Initiative, was featured in Journal of Chemical Physics.

\section{Citation:}

Yoo S, XC Zeng, and SS Xantheas. 2009. "On the Phase Diagram of Water with Density Functional Theory Potentials: The Melting Temperature of Ice $\mathrm{I}_{h}$ with the Perdew-Burke-Ernzerhof and Becke-LeeYang-Parr Functionals." Journal of Chemical Physics 130:221102. 


\title{
In Situ Studies of Soft- and Reactive Landing of Mass-Selected lons Using Infrared Reflection Absorption Spectroscopy
}

\author{
Q Hu, ${ }^{(a)} P$ Wang, ${ }^{(a)} P L$ Gassman, ${ }^{(a)}$ and J Laskin ${ }^{(a)}$ \\ (a) Pacific Northwest National Laboratory, Richland, Washington
}

For the first time, researchers can determine if large molecules and other charged particles that softly land on a surface form the desired bonds, thanks to a new approach built by a team of EMSL users at Pacific Northwest National Laboratory. This method, developed using EMSL capabilities, relies on in situ reflection-absorption spectroscopy to monitor the formation of a chemical bond between the ion and the surface. In designing specialized sensors and other technologies, scientists want to prepare customized layers of molecules on specific surfaces. To build these layered materials, scientists must first understand the reactions involved. This new tool provides vital insights on the reactivity between ions and surfaces. Using it, scientists can study bond formation and the impact of the surface on the secondary structure and orientation of large molecules that land on it.

Using this new approach (Figure 1), the team made a startling discovery about the efficiency of bond formation between the ion and the surface. Using diamine, a linear molecule with nitrogen atoms at each end, as a model ion, they found that nearly every ion that hit the surface bonded to it - a very surprising result.

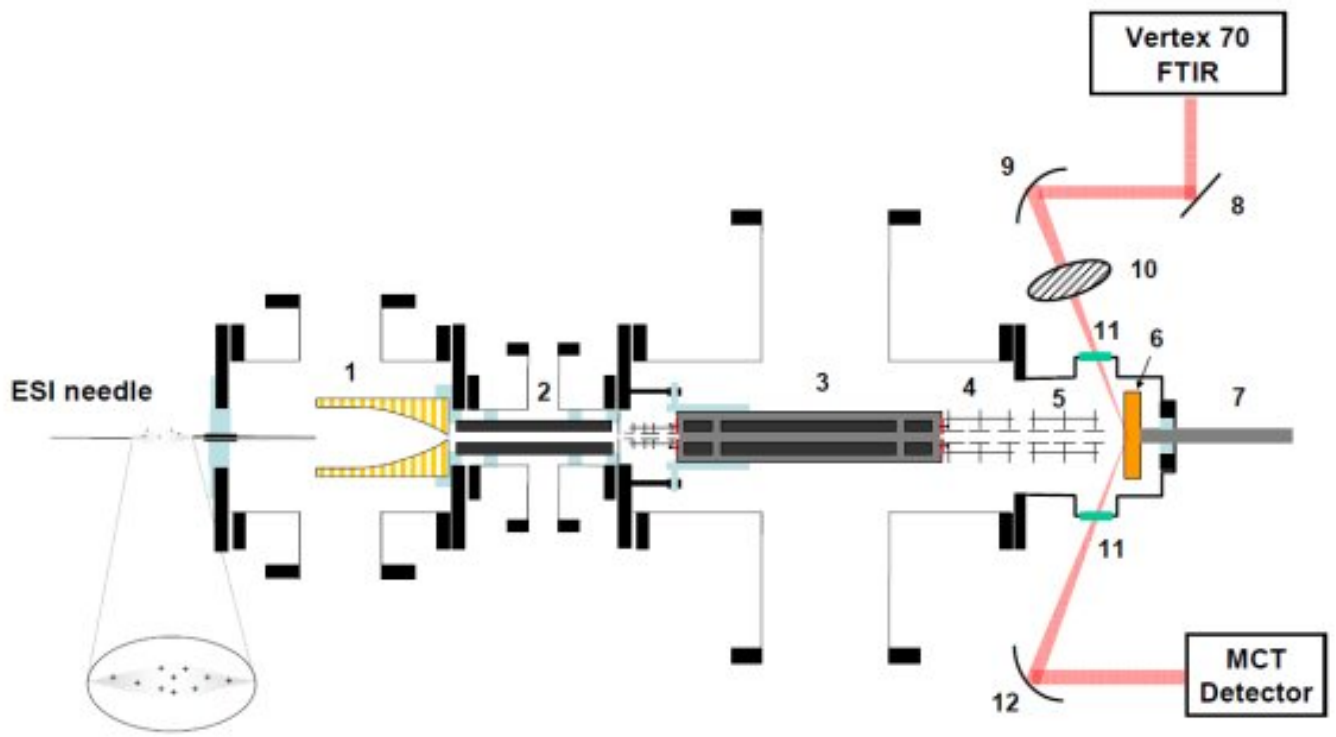

Figure 1. Scientists can now determine if ions form the desired bonds to a surface, thanks to a modified ion soft landing instrument. The schematic shows (1) ion funnel, (2) collisional quadrupole, (3) mass filter, (4) and (5) two lenses, (6) surface, (7) linear motion feedthrough for positioning of the surface, (8) flat mirror, (9) and (12) parabolic mirrors, (10) MIR wire grid polarizer, (11) viewports.

To study bond formation between ions and reactive surfaces, researchers previously had to expose surfaces to a beam of mass selected ions, then take the substrate outside the vacuum system and perform the analysis. But, as a general rule, researchers prefer to study reactions as they happen as exposure of surfaces to laboratory air can alter the structure of deposited molecules and introduce artifacts. 
The researchers selected for their experiments a long chain-like molecule, with 12 carbon atoms in the middle and an amine group on each end. Ions of this molecule were selected using a mass filter and were sent to react with a carbon-based surface by forming an amide bond. This bond involves a carbonyl group $(\mathrm{C}=\mathrm{O})$ on the substrate and an amine group $\left(\mathrm{NH}_{2}\right)$ of the ion each shedding off other atoms and bonding together. Surprisingly, the researchers found that nearly every molecule that hit the surface bonded. In the past, studying bond formation between small proteins or peptides and surfaces using infrared spectrometry was difficult because the signals indicating the formation of a new amide bond between the surface and the protein were buried under strong infrared bands of the protein itself. Now, scientists can study the reactions as they occur. The new approach allows researchers to unambiguously prove that bond formation indeed takes place and quantify the amount of amide bonds formed.

However, initial bond formation just one part of a bigger picture. Researchers can also study how the surface affects the material that land on it. For example, placing a large molecule that is happy in water on a surface that repels water will cause the molecule to reorganize. Because the conformation of a large molecules determines its function, understanding this phenomenon is essential for designing nanomaterials with controlled properties. This new technique will help answer questions about how such rearrangements occur.

In the future studies, the scientists will examine the effect of the substrate on the orientation and conformation of soft-landed peptides and proteins. In addition, they are continuing their work to understand the interactions between molecules and surfaces to arrive at the time when understanding leads to controlled preparation of functional materials. Further, with this new method available at EMSL, scientists from around the world have another tool in their arsenal to study bond formation. Already, a scientist from the University of Arizona, through EMSL's rapid access program, is using the approach for his studies.

This research, funded by DOE's Office of Basic Energy Sciences, was featured in Analytical Chemistry.

\title{
Citation:
}

Hu Q, P Wang, PL Gassman, and J Laskin. 2009. "In Situ Studies of Soft- and Reactive Landing of MassSelected Ions Using Infrared Reflection Absorption Spectroscopy." Analytical Chemistry. 81:7302-7308. DOI: $10.1021 /$ ac901149s.

\section{An Isotropic Chemical Shift-Chemical Shift Anisotropic Correlation Experiment using Discrete Magic Angle Turning}

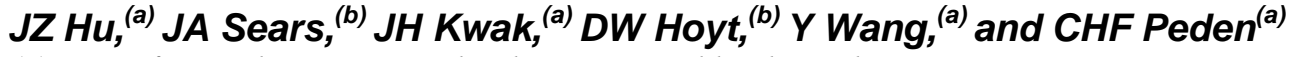 \\ (a) Pacific Northwest National Laboratory, Richland, Washington \\ (b) EMSL, Richland, Washington
}

A new nuclear magnetic resonance capability developed at EMSL—called DMAT—will allow researchers to gain new insights into catalytic reactions, which may lead to an improved ability to tailor catalysts for optimal performance for applications such as alternative energy production and environmental remediation. In addition, DMAT can be used to collect novel data for-and, therefore foster a stronger fundamental understanding of-biological systems, such as dense cell systems and cells attached to solid surfaces. 
The DMAT (Figure 1), or discrete magic angle turning, apparatus and method was developed by researchers at the Pacific Northwest National Laboratory's Institute for Interfacial Catalysis and EMSL, and merited a United States patent.

DMAT combines the advantages of two established NMR technologies: magic angle hopping and magic angle turning. MAH and MAT are built from the cornerstone of NMR, magic angle spinning, which produces high-resolution, high-sensitivity isotropic spectra. But traditional MAS at a sample spinning rate of several $\mathrm{kHz}$ cannot be adapted for in situ investigations that require simultaneous, elevated pressure and flow controls. MAH allows simultaneous control over sample conditions (pressure, flow, and temperature) and generates enhanced structural data, but technical difficulties limit its wide-spread application for in situ experiments. MAT overcomes some of the technological challenges of MAH, but cannot be applied to in situ investigations that require precise pressure control. DMAT combines the best of both worlds of MAH and MAT and showed great potential in preliminary experiments. For example, DMAT structural data on 1,2,3-trimethoxybenzene were significantly enhanced compared to MAH and similar to that obtained with

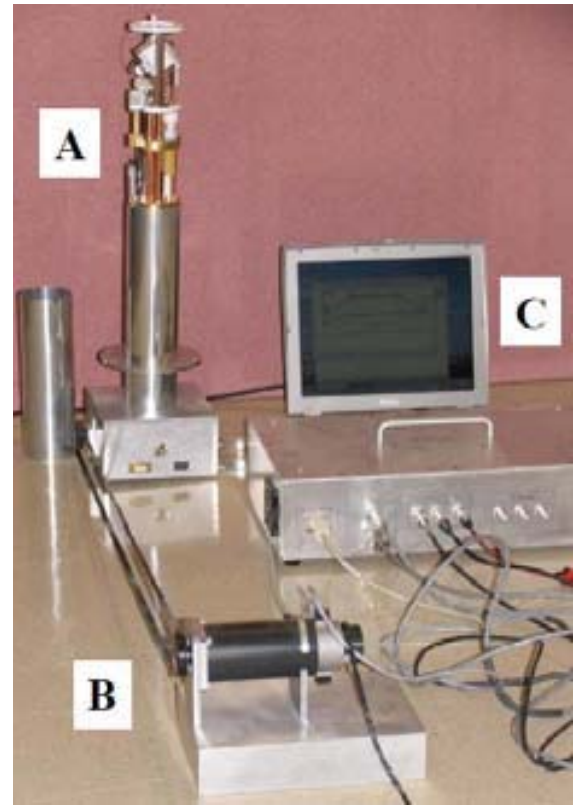

Figure 1. The DMAT apparatus consists of three parts: a specialized NMR probe (A), a DC Servo motor (B), and a computer-programmed box that precisely controls the rotation of the $\operatorname{motor}(C)$. MAT, and this data quality was coupled with the ability to control sample temperature, pressure, flow conditions, and reactant feed composition in situ and simultaneously.

The simultaneous in situ control over sample conditions afforded by DMAT will open new doors for researchers to conduct in situ NMR studies of catalytic reactions and biological systems. The invention garnered a U.S. patent, Discrete Magic Angle Turning System, Apparatus, and Process for in situ Magnetic Resonance Spectroscopy and Imaging, Patent Number US 7,535,224 B2, and supports EMSL's goals to characterize surfaces and interfaces with unprecedented resolution as well as to predict biological functions from molecular and chemical data.

This work was supported by the DOE Office of Basic Energy Sciences and by EMSL's Intramural Research Program. It was featured in Journal of Magnetic Resonance.

\section{Citation:}

Hu JZ, JA Sears, JH Kwak, DW Hoyt, Y Wang, CHF Peden. 2009. “An Isotropic Chemical ShiftChemical Shift Anisotropic Correlation Experiment using Discrete Magic Angle Turning." Journal of Magnetic Resonance 198:105-110. 


\title{
Defining the Active Catalyst Structure and Reaction Pathways from $A b$ initio Molecular Dynamics and Operando XAFS: Dehydrogenation of Dimethylaminoborane by Rhodium Clusters
}

\author{
R Rousseau, ${ }^{(a)}$ GK Schenter, ${ }^{(a)}{ }^{2}$ L Fulton, ${ }^{(a)}$ JC Linehan, $^{(a)}$ MH Engelhard, ${ }^{(b)}$ and T Autrey ${ }^{(a)}$ \\ (a) Pacific Northwest National Laboratory, Richland, Washington \\ (b) EMSL, Richland, Washington
}

To use hydrogen as a clean energy source, some engineers want to pack hydrogen into a larger molecule, rather than compressing the gas into a tank. A gas flows easily out of a tank, but getting hydrogen out of a molecule requires a catalyst. Now, researchers reveal new details about one such catalyst. The results are a step toward designing catalysts for use in hydrogen energy applications such as fuel cells.

EMSL researchers and users

Pacific Northwest National

Laboratory combined experimental and theoretical studies to identify the characteristics of the catalyst, a cluster of rhodium, boron and other atoms. The catalyst chemically reacts with ammonia borane, a molecule that stores hydrogen densely, to release the hydrogen as a gas (Figure 1). Their results, which reveal many molecular details of this catalytic reaction, was featured in Journal of the American Chemical Society.

Researchers and engineers are trying to create a hydrogen fuel system that stores hydrogen safely

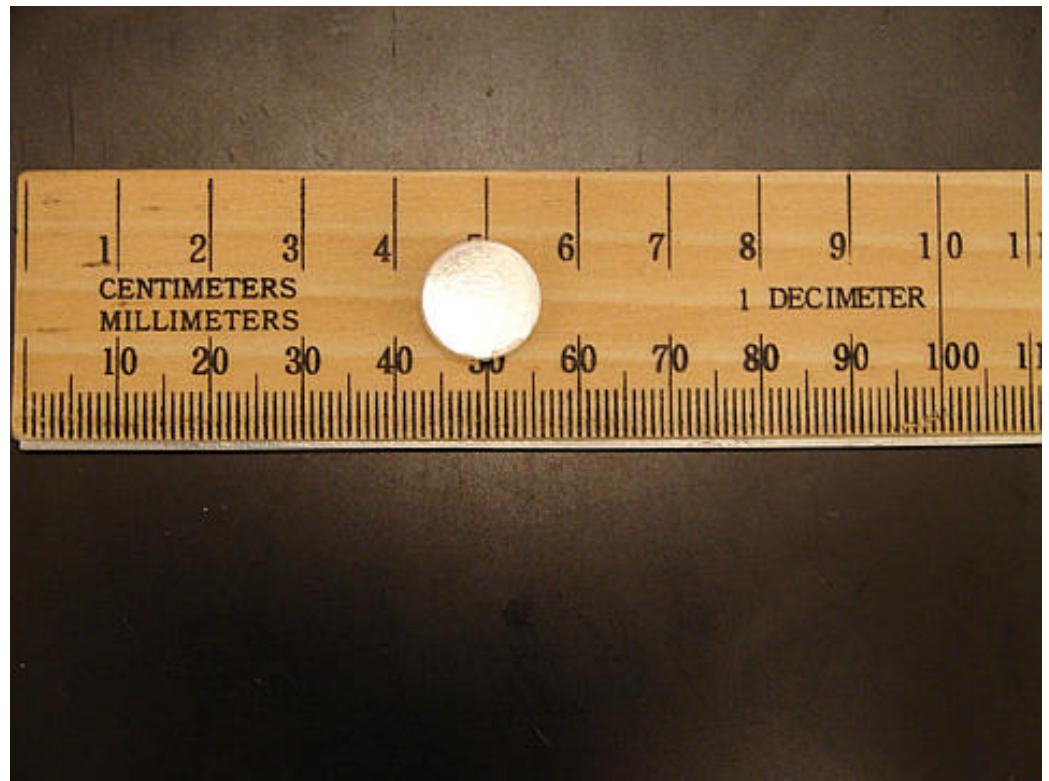

Figure 1. A small pellet of solid ammonia borane $(240 \mathrm{mg})$, as shown, is capable of storing relatively large quantities of hydrogen ( 0.5 liter) in a very small volume.

and discharges hydrogen easily, which can then be used in fuel cells or other applications. One way to achieve such a fuel system is by "storing" hydrogen as part of a larger molecule. The molecule that contains hydrogen atoms, in this case ammonia borane, serves as a sort of structural support. The catalyst plucks the hydrogen from the ammonia borane as needed to run the device.

The researchers study a rhodium-based catalyst that performs this job fairly well, but might have potential for improvement. Their initial work showed that the catalyst worked as a molecule that contained a core of four rhodium atoms in a tetrahedron, or a triangular pyramid, with each corner decorated with boron and other elements. But the rhodium and other atoms could line up in dozens of configurations in the molecule. That wasn't enough information for design improvements - the team wanted to know which of the multitude of structures was the real catalyst, as well as how the atoms worked together to remove the hydrogen from ammonia borane. To find out, the researchers had to combine experimental work with theoretical work, because neither method was sufficient on its own. 
First, the team followed the catalyst-ammonia borane reaction with several technologies. One of the most important is an uncommon technique known as operando XAFS, which allowed them to take X-ray snapshots of the catalyst in action. Most researchers examine a catalyst's structure when the catalyst is at a standstill, but that is like trying to figure out how an athlete performs by watching him sleep. Additional experiments were performed in EMSL. The data from the various experiments were like puzzle pieces that the team had to fit together.

To put the puzzle together, the team used computer models to construct a theoretical molecular configuration that accounted for all the data. These computationally challenging models were calculated on computers at the National Energy Research Scientific Computing Center at Lawrence Berkeley National Laboratory in Berkeley, California. The computer model produced a structure that best incorporated the experimental data. To test whether this structure worked properly, the team performed a computer simulation of an operando XAFS analysis of that catalytic structure reacting with ammonia borane. Then they compared the simulated data with real data gathered about the catalyst. The two sets of data matched very well, suggesting the structure they had come up with was very close to reality.

The chemical nature of the structure, along with additional experimental data, allowed the team to outline the chemical reaction occurring between the catalyst and the ammonia borane. The catalyst does not remain still, making it a good catalyst but, like an active two-year old, also a difficult subject to pin down.

The results suggested that the active catalyst picks off hydrogen from a particular spot on the ammonia borane molecule: a nitrogen atom in the molecule holding onto two hydrogen atoms. First, the catalyst plucks one hydrogen atom off. This is the hardest part of the reaction and it makes the bond between the remaining hydrogen and boron unstable. So the molecule spits off the second hydrogen as well, and the two hydrogen atoms form molecular hydrogen, or $\mathrm{H}_{2}$ which is released as a gas and can be used in engines or fuel cells.

Additional details remain to be drawn out by the team, but this study makes a big dent in what they need to know to design a good, inexpensive catalyst.

This work was supported by DOE's Basic Energy Sciences Program and was featured in the Journal of the American Chemical Society.

\section{Citation:}

Rousseau R, GK Schenter, JL Fulton, JC Linehan, MH Engelhard, and T Autrey. 2009. "Defining the Active Catalyst Structure and Reaction Pathways from Ab initio Molecular Dynamics and Operando XAFS: Dehydrogenation of Dimethylaminoborane by Rhodium Clusters." Journal of the American Chemical Society DOI 10.1021/ja901480u. 


\title{
Low-Energy Networks of the T-Cage $\left(\mathrm{H}_{2} \mathrm{O}\right)_{24}$ Cluster and Their Use in Constructing Periodic Unit Cells of the Structure I (sl) Hydrate Lattice
}

\author{
$S$ Yoo, ${ }^{(a)}$ MV Kirov, ${ }^{(b)}$ and SS Xantheas ${ }^{(a)}$ \\ (a) Pacific Northwest National Laboratory, Richland, Washington \\ (b) Siberian Branch of the Russian Academy of Sciences, Tyumen, Russia
}

There's a lot more to water than meets the eye. Water molecules form threedimensional empty cages organized in a lattice with the potential to store large amounts of natural gases. Gases such as hydrogen, methane, and carbon dioxide can be fit as "guest" molecules inside these "host" cavities. But water molecules can be arranged in millions of ways in the basic unit cell, and scientists need to know those arrangements that are energetically more stable compared to others, because lower-energy, stable arrangements have the greatest potential for storing energy. With millions of possible combinations, finding the ones that are lower in energy becomes a challenge of staggering proportions. Now a computer model built by EMSL users from the Pacific Northwest National Laboratory and the

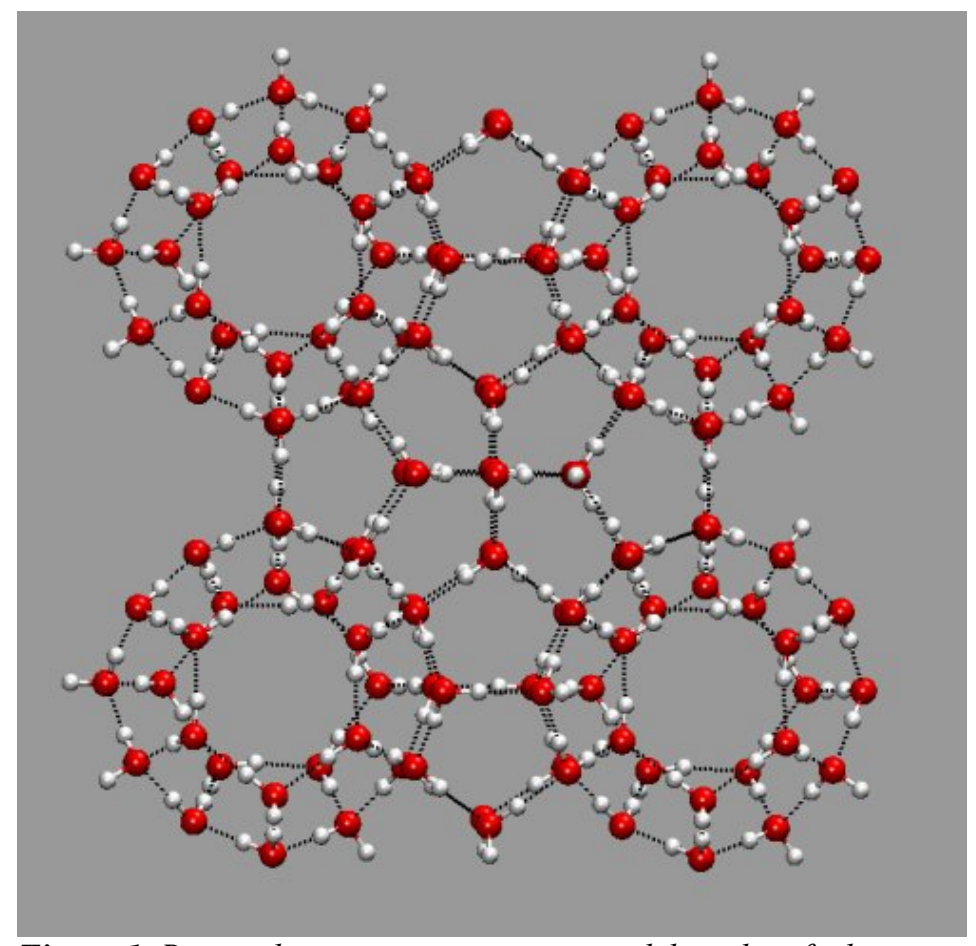

Figure 1. Researchers use an innovative model to identify the most stable water molecule lattices for energy storage.

Russian Academy of Sciences allows scientists to rapidly pinpoint the most stable structures and use them to model those systems that hold promise for energy storage (Figure 1).

At high pressures and low temperatures, like those found in permafrost or on the ocean's floor, water molecules organize into complex hydrate lattices that are held together by hydrogen bonds. Because these lattices are so complex, even sophisticated computer models struggle to find the lattices of most interest to researchers.

Previous methods used to analyze lattices relied on a "top down" approach. Under those methods, researchers started with the known arrangement of the oxygen atoms and randomly selected the positions for the hydrogen atoms to build a network that obeyed certain rules. That approach had several drawbacks. It was based on statistical sampling, which required very large unit cells to be effective. Then too, the resulting structures could be more theoretical than practical, meaning they might not exist in reality. Even more problematic, the model couldn't guarantee that the most appropriate structures would even be found. 
The new approach starts from the bottom, by looking at the building blocks of the hydrate lattices and the characteristics needed for stability. Researchers then use these blocks to build the more complex lattices. This approach ensures that the resulting structures meet all requirements for stability.

The computer model screens quickly through all possible configurations of the water cages that are used to build the hydrate lattice by calculating the energy necessary to hold each collection of water molecules together. Some arrangements require very large amounts of energy to make. These are discarded. Others need less energy to build. These are most likely the arrangements that would actually exist.

PNNL chemist Dr. Sotiris S. Xantheas and colleagues Dr. Soohaeng Yoo, also at PNNL, and Dr. Mikhail Kirov of the Russian Academy of Sciences had previously applied this approach on a smaller arrangement consisting of a hollow cage of 20 water molecules. They narrowed an initial list of 30,026 possible arrangements down to 64 , which were most likely to be the more stable ones. The most recent calculations involved hollow cages of 24 water molecules. The model refined the list of over three million structures down to just 321. With the use of the supercomputer at EMSL, the team performed detailed electronic structure calculations to further refine those arrangements. The researchers were then able to computationally build up the unit cells into a three-dimensional structure I (sI) hydrate lattice that is capable of holding the alternative energy source hydrogen as well as natural gases.

The work provides realistic models for the hydrate lattices and allows researchers to understand the underlying intermolecular interactions that are responsible for storing "guest" molecules in these "host" lattices.

Water molecules form hydrate lattices in three types of structures, labeled sI, sII, and sH. The current work modeled the sI hydrate lattice. In the future, the researchers hope to model the sII hydrate lattice, for which the unit cell is made from 16 units of hollow cages with 20 water molecules each and 8 units of an even larger hollow cage of 28 water molecules that can have over 60 million possible arrangements. The (sII) hydrate has been reported to meet current Department of Energy's target densities for an onboard hydrogen storage system.

DOE's Office of Basic Energy Sciences and the Russian Academy of Sciences funded the research, which was featured in the Journal of the American Chemical Society.

\section{Citation:}

Yoo S, MV Kirov, and S Xantheas. "Low-Energy Networks of the T-Cage $\left(\mathrm{H}_{2} \mathrm{O}\right)_{24}$ Cluster and Their Use in Constructing Periodic Unit Cells of the Structure I (sI) Hydrate Lattice." Communication to the Editor, Journal of the American Chemical Society 131(22):7564-7566. 



\section{Fiscal Year 2009 User Projects}

(gc3564)Nanostructure Formation, Aggregation, and Reactivity

S Irle, Z Wang, B Finck, PN Bobadova-parvanova, A Altun, I Kerkines-Keramidas, J Jakowski, Emory University, Atlanta, Georgia

K Morokuma, Kyoto University, Kyoto, Japan

R Rousseau, M Dupuis, X Wang, SN Kerisit, D Mei, Pacific Northwest National Laboratory, Richland, Washington

F Mehmood, LA Curtiss, P Zapol, SP Adiga, MG Sternberg, H Iddir, JP Greeley, D Torres Rangel, H He, RB Rankin, Argonne National Laboratory, Argonne, Illinois

MA Langell, University of Nebraska, Lincoln, Nebraska

M Valiev, EJ Bylaska, N Govind, Environmental Molecular Sciences Laboratory, Richland, Washington

G Zheng, University of Illinois at Urbana-Champaign, Urbana, Illinois

NA Deskins, Worcester Polytechnic Institute, Worcester, Massachusetts

3D Imaging of Organic Nanoparticle Fillers in Organic Matrices

J Simonsen, Oregon State University, Corvallis, Oregon

3-Dimensional Reconstruction and Modeling of Bacterial Extracellular Polymeric Substances (ExPS)

MJ Marshall, Pacific Northwest National Laboratory, Richland, Washington

A Dohnalkova, Environmental Molecular Sciences Laboratory, Richland, Washington

43Ca NMR Spectroscopy of Ca2+ Dependent Proteins and models

AS Lipton, PD Ellis, R Heck, Pacific Northwest National Laboratory, Richland, Washington

JV Hanna, University of Warwick, Coventry, United Kingdom

51V Solid-State MAS NMR Studies of Vanadium Haloperoxidases

S Bolte, KJ Ooms, TE Polenova, University of Delaware, Newark, Delaware

57Fe-Mossbauer Studies of Sediment Cores From Integrated Field-Scale Subsurface Research Challenge Site at Rifle, Colorado

NP Qafoku, Pacific Northwest National Laboratory, Richland, Washington

R Kukkadapu, Environmental Molecular Sciences Laboratory, Richland, Washington

A Community Modeling Framework to Advance Numerical Treatments of Aerosol Processes WI Gustafson, EG Chapman, JD Fast, Pacific Northwest National Laboratory, Richland, Washington M Shrivastava, PNNL, Richland, Washington

A Hyperbaric AFM for Imaging Mineral Surfaces in Supercritical CO2

AS Lea, JE Amonette, Pacific Northwest National Laboratory, Richland, Washington 
A Metabolomic Approach For Characterizing Methylenetetrahydrofolate Reductase Mutations And Possible Outcomes For The Treatment of Related Diseases

K McAteer, Washington State University, Richland, Washington

HD Hamilton, Pacific Northwest National Laboratory, Richland, Washington

AC Swensen, Washington State University Tri-Cities, Richland, Washington

KM Meyer, PNNL, Richland, Washington

A Model System to Obtain Structural Information From the Bacterial-Mineral Interface by Neutron Reflectometry

A Johs, L Liang, Oak Ridge National Laboratory, Oak Ridge, Tennessee

A Nanoconfined Plasmonic Light Source for Imaging and Spectroscopy With Ultrahigh Spatial and Temporal Resolution

MB Raschke, RL Olmon, EE Josberger, BS Burkholder, University of Washington, Seattle, Washington

A Periodic Density Functional Study of VOx Clusters Supported on the Defected Anatase TiO2 (101) Surface

AT Bell, V Shapovalov, University of California, Berkeley, Berkeley, California

A Proteomic Analysis of the Effects of Environmental Factors on Hydrogen Production in the Cyanobacterium Anabaena Variabilis.

T Thiel, University of Missouri, St. Louis, Saint Louis, Missouri

A Proteomic Dissection of the Hg(II) Toxicity Paradox

SM Miller, University of California, San Francisco, San Francisco, California

AO Summers, University of Georgia, Athens, Georgia

A Proteomic Investigation of Changes in Lipid Droplet-Bound Proteins

SP Gross, University of California, Irvine, Irvine, California

A Proteomic Perspective of Biodiversity

SJ Callister, Pacific Northwest National Laboratory, Richland, Washington

JJ Parnell, ME Pfrender, Utah State University, Logan, Utah

A Systematic Molecular Modeling Study of the Effect of Lipid Bilayer Composition on Resistance to Alcohol-Induced Changes

R Faller, MI Hoopes, JM Vanegas, University of California, Davis, Davis, California

A Systems Biology Approach to Emerging Respiratory Viral Diseases

TO Metz, UK Aryal, RD Smith, Pacific Northwest National Laboratory, Richland, Washington

MG Katze, GL Law, University of Washington, Seattle, Washington

JR Teuton, PNNL, Richland, Washington

A Systems Biology Approach to Infectious Diseases Research

K McAteer, Washington State University, Richland, Washington

JN Adkins, R Heck, JR Cort, GW Buchko, TO Metz, C Ansong, Pacific Northwest National Laboratory, Richland, Washington

VL Motin, University of Texas Medical Branch, Galveston, Texas 
BO Palsson, University of California, San Diego, La Jolla, California

F Heffron, Oregon Health Sciences University/Oregon Graduate Institute, Portland, Oregon

SN Peterson, J. Craig Venter Institute, Rockville, Maryland

\section{A Systems Biology Approach to Infectious Diseases Research for EnteroPathogens}

JN Adkins, R Heck, RN Brown, C Ansong, S Chowdhury, MC Burnet, JE Mcdermott, GW Buchko, DN

Sharp, JL Martin, AC Rutledge, Pacific Northwest National Laboratory, Richland, Washington

VL Motin, University of Texas Medical Branch, Galveston, Texas

HM Brewer, Environmental Molecular Sciences Laboratory, Richland, Washington

BO Palsson, University of California, San Diego, La Jolla, California

F Heffron, Oregon Health Sciences University/Oregon Graduate Institute, Portland, Oregon

SN Peterson, J. Craig Venter Institute, Rockville, Maryland

Abiotic Reduction of U(VI) by Sorbed Fe(II) and the Implications for Field-Scale Bioremediation at the Rifle IFC Project Site

J Davis, KM Campbell, US Geological Survey, Menlo Park, Menlo Park, California

\section{Absorption and Emission of Radiation in Materials}

Y Houndonougbo, Eastern Washington University, Cheney, Washington

SH Garofalini, Rutgers University, Piscataway, New Jersey

SM Kathmann, R Devanathan, RM Van Ginhoven, Pacific Northwest National Laboratory, Richland,

Washington

PA Moreira, Universidade Estadual De Campinas, Sao Paulo, Brazil

JD Musgraves, LR Corrales, OL Monti-masel, B Potter, MH De Silva, H Chandra, LK Schirra, BL

Mooney, EA Randtke, ML Blumenfeld, B Kim, AJ Higgins, University of Arizona, Tucson, Arizona

Accelerated Fuel-Cladding Interaction Study using Surrogate Materials

CH Henager, C Windisch, Pacific Northwest National Laboratory, Richland, Washington

MH Engelhard, C Wang, Environmental Molecular Sciences Laboratory, Richland, Washington

Accurate Ab Initio Determinations of Thermochemistry of Novel Molecular Radicals, Cations and Neutrals of Relevance to Combustion, Environmental, and Atmospheric Chemistry

$\mathrm{C} \mathrm{Ng}$, C Lam, C Houchins, University of California, Davis, Davis, California

K Lau, City University of Hong Kong, Kowloon, Hong Kong, Hong Kong

S Chiu, National Center for Supercomputing Applications, Urbana, Illinois

Accurate Embedded-Cluster Modeling of Insulators: Applications to Metal Oxide Surface Chemistry \& Surface Excitation Processes

KA Lopata, University of California, Los Angeles, Los Angeles, California

PV Sushko, London, University College, London, United Kingdom

Z Dohnalek, AG Joly, R Rousseau, Pacific Northwest National Laboratory, Richland, Washington

N Govind, K Kowalski, Environmental Molecular Sciences Laboratory, Richland, Washington

MJ Wolf, University College London, London, United Kingdom

Activation and Dissociation of Large Molecules in Mass Spectrometry

J Laskin, Q Hu, Pacific Northwest National Laboratory, Richland, Washington

JS Smith, University of Washington, Seattle, Washington 
Z Yang, University of Colorado Boulder, Boulder, Colorado

GE Johnson, PJ Roach, PNNL, Richland, Washington

Active Standoff Detection of Low-Volatility Chemicals on Soils Using Infrared ReflectionAbsorption Spectroscopy (NNSA, PNNL Scope \#25399)

TA Blake, Pacific Northwest National Laboratory, Richland, Washington

PL Gassman, Environmental Molecular Sciences Laboratory, Richland, Washington

Adhesion of Polymer Spheres to Modified Natural Fibers

JD Holbery, D Howe, C Huang, Pacific Northwest National Laboratory, Richland, Washington

PL Gassman, MH Engelhard, B Arey, Environmental Molecular Sciences Laboratory, Richland, Washington

Advanced Biomolecular Simulations - Development and Applications

C Wu, GR Kulkarni, Washington State University, Pullman, Washington

TP Straatsma, TA Soares Da Silva, WR Cannon, MM Rawlins, RD Lins, Pacific Northwest National

Laboratory, Richland, Washington

DJ Baxter, Environmental Molecular Sciences Laboratory, Richland, Washington

RC Wade, R Gabdoulline, P Mereghetti, EML Research gGmbH, Heidelberg, Germany

MW Zacharias, S Kannan, Jacobs University Bremen, Bremen, Germany

Advanced Diagnostic Studies of Lithium Ion Batteries Using In Situ and Ex Situ NMR Spectroscopies

J Hu, L Wang, J Zhang, Z Yang, J Liu, D Choi, dn wang, V Murugesan, Pacific Northwest National

Laboratory, Richland, Washington

J Sears, Environmental Molecular Sciences Laboratory, Richland, Washington

Advanced IMPROVE Studies

DH Lowenthal, Desert Research Institute, Reno, Nevada

Advanced Peta-Scale Molecular Dynamics Simulations

TP Straatsma, J Nieplocha, BJ Palmer, EG Stephan, TA Soares Da Silva, CL Baird, DO Apiyo, Pacific

Northwest National Laboratory, Richland, Washington

MS Sansom, University of Oxford, Oxford, United Kingdom

JW Essex, University of Southampton, Southampton, United Kingdom

RC Wade, V Cojocaru, EML Research gGmbH, Heidelberg, Germany

MW Zacharias, Jacobs University Bremen, Bremen, Germany

Advanced Proteomics and Metabolomics Studies of Type 1 Diabetes

TO Metz, JM Jacobs, DG Camp, GI Mezengie

Pacific Northwest National Laboratory, Richland, Washington

Advances in Understanding Electron Transfer Kinetics at Environmental Interfaces

AL Neal, University of Georgia, Aiken, South Carolina

KM Rosso, SN Kerisit, Z Wang, ES Ilton, S Yanina, TC Droubay, PP Zarzycki, J Liu, Pacific Northwest

National Laboratory, Richland, Washington

DM Smith, Whitman College, Walla Walla, Washington 
CS Lo, Washington University in St. Louis, St. Louis, Missouri

J Boily, Umea University, Umea, Sweden

CI Pearce, PNNL, Richland, Washington

Age Related Post Translational Modifications to Proteins That Alter Protein Complexes Determined by FTICR-MS

HS Smallwood, St. Jude Children's Research Hospital, Memphis, Tennessee

Airborne Measurement of Dimethylsulfide, Isoprene, and other Trace Volatile Organic Compounds using a Proton Transfer Reaction Mass Spectrometer during the 2008 DOE ASP VOCALS Field Campaign

Y Lee, Brookhaven National Laboratory, Upton, New York

L Alexander, Environmental Molecular Sciences Laboratory, Richland, Washington

Alpha Project Phosphoproteomics

DG Camp, RA Maxwell, RD Smith, Pacific Northwest National Laboratory, Richland, Washington

O Resnekov, Molecular Sciences Institute, Berkeley, California

Amorphous Semiconductor Analysis Using Ion Beam Tools

CH Henager, J Ryan, BR Johnson, Pacific Northwest National Laboratory, Richland, Washington

An Experimental and Data Analysis Investigation Into the Trapped Gases and Non-Ice Material in the Surfaces of the Outer Planets Icy Satellites and Our Moon

CA Hibbitts, Johns Hopkins University, Laurel, Maryland

J Szanyi, Pacific Northwest National Laboratory, Richland, Washington

An Integrated Approach to Quantifying the Coupled Biotic and Abiotic Mechanism, Rates and Long-Term Performance of Phosphate Barriers for In Situ Immobilization of Uranium DM Wellman, A Miracle, EM Pierce, E Richards, MA Covert, TE Queen, M Oostrom, G Tartakovsky, Pacific Northwest National Laboratory, Richland, Washington JB Nelson, Whitman College, Walla Walla, Washington

TW Wietsma, Environmental Molecular Sciences Laboratory, Richland, Washington M Sinha, PNNL, Richland, Washington

An Integrated Workflow for Identification and Quantitation of Intact Phosphoproteins EW Robinson, ZM Tian, F Yang, DL Stenoien, Pacific Northwest National Laboratory, Richland, Washington

N Tolic, HM Brewer, R Zhao, L Pasa-Tolic, Environmental Molecular Sciences Laboratory, Richland, Washington

An NMR Study of the Complex Formed by Viral Protein Vcci and Human Chemokine Eotaxin W Tian, PJ LiWang, L Zhang, Texas A\&M University, College Station, Texas

Analysis of Metabolic Responses to Signals From the Oncogene C-Myc a Regulator of Cell Growth and Proliferation.

NG Isern, Environmental Molecular Sciences Laboratory, Richland, Washington

FM Morrish, DM Hockenbery, Fred Hutchinson Cancer Research Center, Seattle, Washington 
MA Portman, Children's Hospital and Regional Medical Center, Seattle, Washington

\section{Analysis of POSIX I/O Extensions}

RJ Latham, Argonne National Laboratory, Argonne, Illinois

EJ Felix, Environmental Molecular Sciences Laboratory, Richland, Washington

\section{Analysis of Self-Assembled Monolayers on Mesoporous Supports (SAMMS) Materials}

JT Bays, RJ Wiacek, RS Addleman, A Cinson, J Davidson, ca fernandez, Pacific Northwest National Laboratory, Richland, Washington

\section{Analysis of Trout Sperm Nuclear Proteins}

J Nagler, University of Idaho, Moscow, Idaho

IR Schultz, Pacific Northwest National Laboratory, Richland, Washington

\section{Anchoring Oxidation Catalysts inside Hollow Large-Pore Nanospherical Silica Materials}

RL Luck, Michigan Technological University, Houghton, Michigan

\section{Application of High-Throughput Proteomics}

SJ Giovannoni, Oregon State University, Corvallis, Oregon

SB Levy, Tufts University School of Medicine, Boston, Massachusetts

L Shapiro, E Toro, LC Britos, Stanford University, Stanford, California

MS Lipton, JE Turse, E Zink, BM Ham, JC Scholten, T Taverner, H Zhang, BL Champion, KE Burnum, Pacific Northwest National Laboratory, Richland, Washington

TJ Donohue, University of Wisconsin-Madison, MADISON, Wisconsin

A Tsapin, University of Southern California, Los Angeles, California

T Mester, University of Massachusetts, Amherst - Biochemistry and Molecular Biology, Amherst,

Maryland

DR Lovley, University of Massachusetts Amherst, Amherst, Massachusetts

S Kaplan, UT-Houston Medical School, Houston, Texas

\section{Application of Support Vector Machine approach to Chinook failure prediction}

GA Fink, Pacific Northwest National Laboratory, Richland, Washington

DE Cowley, Environmental Molecular Sciences Laboratory, Richland, Washington

EW Fulp, RW Featherstun, Wake Forest University, Winston-Salem, North Carolina

\section{Assembly of the mRNA 3'-end processing machinery}

G Varani, TC Leeper, DW Begley, MF Bardaro, PL Hsu, University of Washington, Seattle, Washington

\section{Associate Professor of Chemistry}

RL Luck, Michigan Technological University, Houghton, Michigan

\section{Atomically Resolved Studies of Catalytic and Photocatalytic Processes on TiO2(110)}

S Li, Tulane University, New Orleans, Louisiana

Z Dohnalek, BD Kay, Z Zhang, R Rousseau, Z Li, Pacific Northwest National Laboratory, Richland,

Washington

JM White, University of Texas at Austin, Austin, Texas 
Basis of Microbial Membrane Attachment to Mineral Surfaces and Ionic Contaminants Uptake BH Lower, Ohio State University, Columbus, Ohio

RD Lins, Pacific Northwest National Laboratory, Richland, Washington

M Valiev, Environmental Molecular Sciences Laboratory, Richland, Washington

\section{Benchmark Activities on Chinook}

MJ Koop, JL Perkins, GJ Marsh, KC Kandalla, H Subramoni, Ohio State University, Columbus, Ohio

ST Elbert, Pacific Northwest National Laboratory, Richland, Washington

JS Welling, Carnegie Mellon University, Pittsburgh, Pennsylvania

KM Regimbal, DE Cowley, TS Carlson, Environmental Molecular Sciences Laboratory, Richland, Washington

\section{Benchmark Modeling of Aerosol-Cloud Interactions}

M Ovtchinnikov, RC Easter, JM Comstock, J Fan, Pacific Northwest National Laboratory, Richland, Washington

A Khain, Hebrew University of Jerusalem, Jerusalem, Israel

\section{Benchmark Non-Born--Oppenheimer Calculations on Small Molecules}

S Bubin, L Adamowicz, University of Arizona, Tucson, Arizona

\section{Biofilm Reactor Development and Characterization Studies}

H Beyenal, RS Renslow, HD Nguyen, A Bulbul, B Cao, Washington State University, Pullman, Washington

Biogeochemical Processes Responsible for the Enhanced Transport of Plutonium under Transient Unsaturated Condtions.

H Nitsche, University of California, Berkeley, Berkeley, California

R Kukkadapu, Environmental Molecular Sciences Laboratory, Richland, Washington

DI Kaplan, Savannah River National Laboratory, Aiken, South Carolina

\section{Biogeochemistry of Oxidation-Reduction Cycling of Uranium and Iron}

WD Burgos, Pennsylvania State University, University Park, Pennsylvania

\section{Bioinformatics Tools to Define the Proteomic State of the Cell}

WR Cannon, GA Anderson, CS Oehmen, KH Jarman, A( Heredia-Langner, DJ Taasevigen, JE

Mcdermott, V Petyuk, Pacific Northwest National Laboratory, Richland, Washington

DJ Baxter, Environmental Molecular Sciences Laboratory, Richland, Washington

JM Malard, SIMUCAD Design Automation, Santa Clara, California

Biological Forensics \& Attribution: Chemical and Physical Analysis â?? Organic Signatures

HW Kreuzer, JJ Moran, J Wahl, KL Wahl, Pacific Northwest National Laboratory, Richland, Washington

MK Newburn, Environmental Molecular Sciences Laboratory, Richland, Washington

Biological Templates $f$ or Synthesis of Ordered Ceramic Nanostructures

Y Shin, G Exarhos, C Windisch, Pacific Northwest National Laboratory, Richland, Washington

B Arey, MH Engelhard, Environmental Molecular Sciences Laboratory, Richland, Washington 
Biomarker Discovery Tools Development

K Petritis, D Lopez-Ferrer, Pacific Northwest National Laboratory, Richland, Washington

\section{Biomarkers of Malaria Infection}

M Fried, Seattle Biomedical Research Institute, Seattle, Washington

\section{Biomineralology and Ultrastructure of Neutrophilic Iron-Oxidizing Bacteria}

KJ Edwards, University of Southern California, Los Angeles, California

CS Chan, Woods Hole Oceanographic Institution, Woods Hole, Massachusetts

\section{Biotemplated Nanoparticles for Imaging and Drug Delivery}

Y Lin, A Ma-ham, Pacific Northwest National Laboratory, Richland, Washington

H Liao, Xiamen University, Xiamen, Fujian, China

K Pinwattana, Chulalongkorn University, Patuwan, Bangkok, Thailand

D Du, Central China Normal University, Wuhan, Hubei, China

S Zhang, Harbin Institute of Technology, Harbin, China

Y Zhang, Hunan Normal University, Changsha, Hunan, China

\section{Bone Growth on Tailored Biomimetic Surfaces}

MI Laitinen, S Sangyuenyongpipat, University of Jyvaskyla, Jyvaskyla, Finland

HJ Whitlow, University of Jyväskylä, University of Jyväskylä, Finland

\section{Bradley Tebo's Proposal}

BM Tebo, WF Smythe, Oregon Health Sciences University/Oregon Graduate Institute, Beaverton, Oregon

\section{Burkholderia Proteomoics}

JE Turse, Pacific Northwest National Laboratory, Richland, Washington

SE Schutzer, University of Medicine and Dentistry, New Jersey Medical School, Newark, New Jersey

JR Teuton, PNNL, Richland, Washington

\section{Capture and Reduction of Metal Ions out of the Environment by Biomolecular Systems}

BH Lower, ES Taylor, Ohio State University, Columbus, Ohio

RD Lins, TP Straatsma, B Clowers, E Baker, TA Soares Da Silva, Pacific Northwest National

Laboratory, Richland, Washington

M Valiev, WA De Jong, Environmental Molecular Sciences Laboratory, Richland, Washington

J Boily, Umea University, Umea, Sweden

DA Dixon, MT Nguyen, VE Jackson, University of Alabama, Tuscaloosa, Tuscaloosa, Alabama

U Rothlisberger, M Guglielmi, EPFL Swiss Federal Institute of Technology, Lausanne, Switzerland

OV Oliveira, Federal University of Sao Carlos, Sao Carlos, Brazil

\section{Catalytic and Optical Properties of Surface Attached and Matrix Embedded Quantum Dots}

G Duscher, North Carolina State University, Raleigh, North Carolina

P Clifton, Imago Scientific Instruments, Madison, Wisconsin 
Catalytic Studies of Transition Metal Oxide Monolayers Supported on Metal Single Crystal Surfaces

Q Ge, Y Pan, Southern Illinois University, Carbondale, Illinois

Z Dohnalek, R Rousseau, Z Zhang, Z Li, Pacific Northwest National Laboratory, Richland, Washington

F Netzer, Karl-Franzens-University Graz, Graz, Austria

Cellular and Molecular Analyses of Dendritic Development in Cultured Neurons

CS Wallace, GS Withers, Whitman College, Walla Walla, Washington

Cellular Response to Virus Infection: Global Proteome Analysis of Macaque Models of HIV and Influenza Infection

MG Katze, DL Diamond, University of Washington, Seattle, Washington

Ceria Nanomonitors for Trace Oxygen Monitoring in Portable Energy Systems

RK Reddy, S Gupta, YS Yadav, VL Venkatraman, DB Meka, Portland State University, Portland, Oregon MH Engelhard, Environmental Molecular Sciences Laboratory, Richland, Washington

S Prasad, RP Sanghavi, Arizona State University, Tempe, Arizona

Chalcogenide Surface Science

BR Johnson, JS McCloy, Pacific Northwest National Laboratory, Richland, Washington

MH Engelhard, Environmental Molecular Sciences Laboratory, Richland, Washington

\section{Characterization of Aerosol Organics by LC-MSn}

A Laskin, Environmental Molecular Sciences Laboratory, Richland, Washington

DH Lowenthal, B Zielinska, V Samburova, AG Hallar, Desert Research Institute, Reno, Nevada

Characterization of Application-Relevant Biosilica-Precipitating Peptides of a Diatom

G Roesijadi, JK Magnuson, Pacific Northwest National Laboratory, Richland, Washington

EV Armbrust, University of Washington, Seattle, Washington

Characterization of Bio-inspired Photonic Crystals for Enhancing Solar Energy Conversion by Single-Molecule Spectroscopy

GL Rorrer, D Gale, Oregon State University, Corvallis, Oregon

D Hu, ME Jones, Pacific Northwest National Laboratory, Richland, Washington

Characterization of Chemistry and Physics at Metal-Polythiophene and Metal-Carbon Interfaces

LM Porter, KA Singh, PB Kulkarni, Carnegie Mellon University, Pittsburgh, Pennsylvania

Characterization of Directly-deposited Germanium Nanostructures

JD Torrey, M Rolandi, SE Vasko, University of Washington, Seattle, Washington

Characterization of Mercury Reduction by Fe(II) Minerals

N Yee, Rutgers University, New Brunswick, New Jersey

R Kukkadapu, Environmental Molecular Sciences Laboratory, Richland, Washington

Characterization of Novel DNA/Semiconductor Nanoconjugates

F Zhou, D Jiang, California State University, Los Angeles, Los Angeles, California 
Characterization of Organic Aerosols throughout the Depth of an Urban Planetary Boundary Layer: proposed field deployment of EMSL mass spectrometers JP Stutz, University of California, Los Angeles, Los Angeles,, California CM Berkowitz, R Zaveri, Pacific Northwest National Laboratory, Richland, Washington R Zhang, Texas A\&M University, College Station, Texas

D Worsnop, JT Jayne, Aerodyne Research Inc, Billerica, Massachusetts

Characterization of Protein Confinement in Functionalized Nanoporous Supports

C Lei, Pacific Northwest National Laboratory, Richland, Washington

Characterization of Solid Oxide Fuel Cell Electrodes After Exposure

O Marina, LR Pederson, GW Coffey, X Zhou, CD Nguyen, AL Schemer-kohrn, Pacific Northwest

National Laboratory, Richland, Washington

MH Engelhard, Environmental Molecular Sciences Laboratory, Richland, Washington

Characterization of Surface Supported Organometallic Olefin Polymerization Catalysts by High Field Solid-State Multi-Nuclear Magnetic Resonance.

AJ Rossini, RW Schurko, University of Windsor, Windsor, Ontario, Canada

Characterization of Synthesised Mixed Valent Natuarlly Occuring Fe-Films

GH Grathoff, University of Greifswald, Portland, Oregon

Characterization of the Local Order of Organic Thin Film Material by Combined Atomic Force Microscopy and Optical Microscopy

D Hu, PE Burrows, AB Padmaperuma, H Qiao, Pacific Northwest National Laboratory, Richland, Washington

PF Barbara, University of Texas at Austin, Austin, Texas

Characterization of the SATB1 Phosphorylations Upon Ionization Radiation

R Zhao, Environmental Molecular Sciences Laboratory, Richland, Washington

T Kohwi-shigematsu, J Li, Lawrence Berkeley National Laboratory, Berkeley, California

Characterization of Unknown Biogenic Organic Compounds During Dissimilatory Metal Reduction PD Majors, DW Kennedy, Pacific Northwest National Laboratory, Richland, Washington

Characterizing Biofilm Development of a Bacterial Biological Control Agent

WF Mahaffee, PK Wallace, Oregon State University, Corvallis, Oregon

Characterizing Biofilm Development of a Bacterial Biological Control Agent

WF Mahaffee, PK Wallace, Oregon State University, Corvallis, Oregon

Characterizing Chemical Composition of DOD-Prescribed Burns

TJ Johnson, L Profeta, RL Sams, Pacific Northwest National Laboratory, Richland, Washington

Characterizing the Effects of Iron- and Sulfate-Reduction on Aquifer Mineralogy: Linking Geophysics and Geomicrobiology

JF Banfield, S Bose, University of California, Berkeley, Berkeley, California

KH Williams, Lawrence Berkeley National Laboratory, Berkeley, California 


\section{Chemical and Physical Properties of Biomass Burning Aerosol}

JL Hand, JL Collett, SM Kreidenweis, Y Dessiaterik, Colorado State University, Fort Collins, Colorado A Laskin, C Wang, Environmental Molecular Sciences Laboratory, Richland, Washington

\section{Chemical Kinetics and Dynamics at Interfaces: Structure and Reactivity of Ices, Amorphous} Solids, and Supercooled Liquid Solutions

J Matthiesen, RS Smith, BD Kay, Z Dohnalek, Pacific Northwest National Laboratory, Richland, Washington

T Zubkov, Ball State University, Muncie, Indiana

\section{Chemistry and Microphysics of Small Particles}

A Zelenyuk, J Yang, T Vaden, Pacific Northwest National Laboratory, Richland, Washington K Mueller, E Nam, H Lee, State University of New York at Stony Brook, Stony Brook, New York

\section{Chemistry of Aerosols in Mexico City: MILAGRO 2006 study.}

A Tivanski, University of Iowa, Iowa City, Iowa

KS Johnson, LT Molina, Massachusetts Institute of Technology, Cambridge, Massachusetts

Y Dessiaterik, Colorado State University, Fort Collins, Colorado

V Shutthanandan, C Wang, Z Zhu, A Laskin, TW Wietsma, Environmental Molecular Sciences

Laboratory, Richland, Washington

MK Gilles, RJ Hopkins, RC Moffet, TR Henn, Lawrence Berkeley National Laboratory, Berkeley, California

Chemistry of Aerosols in the North Slope of Alaska: ISDAC 2008 study.

A Zelenyuk, T Vaden, Pacific Northwest National Laboratory, Richland, Washington

S Moon, Texas A\&M University, College Station, Texas

A Laskin, C Wang, Environmental Molecular Sciences Laboratory, Richland, Washington

MK Gilles, RC Moffet, TR Henn, Lawrence Berkeley National Laboratory, Berkeley, California

\section{Chemistry of Outer Solar System Materials}

TB McCord, GB Hansen, University of Washington, Seattle, Washington

\section{Chlorinated Methane Hydrolysis Rates}

JE Amonette, Pacific Northwest National Laboratory, Richland, Washington

TW Wietsma, Environmental Molecular Sciences Laboratory, Richland, Washington

\section{Chromium Diffusion Kinetics in Nano-Ceria and Doped Ceria Coatings: An UCF-PNNL Collaboration}

S Seal, RK Thanneeru, SA Deshpande, AS Karakoti, University of Central Florida, Orlando, Florida

S Kuchibhatla

Environmental Molecular Sciences Laboratory, Richland, Washington

\section{Cluster Model Studies of Condensed Phase Phenomena}

X Wang, X Xing, W Huang, L Wang, Washington State University, Pullman, Washington

C Ning, Tsinghua University, Beijing, China

PD Dau, Brown University, Providence, Rhode Island

MZ Kamrath, Yale University, New Haven, Connecticut 
C Chi, Fudan University, Shanghai, China

K Matheis, Universitaet Karlsruhe, Karlsruhe, Germany

L Wang, Washington State University Tri-Cities, Richland, Washington

G Xiao, Xi'an Jiaotong University, Xi'an, Shaanxi, China

\section{Clusters of Damage in Irradiated DNA}

MD Sevilla, D Becker, Oakland University, Rochester, Michigan

JD Zimbrick, Colorado State University, Fort Collins, Colorado

MK Bowman, University of Alabama, Tuscaloosa, Tuscaloosa, Alabama

\section{Collaborative Research Projects Supported by the NCRR P41 Resource Center}

SS Rossie, Purdue University, West Lafayette, Indiana

J Shutthanandan, Washington State University, Richland, Washington

DG Moerman, University of British Columbia, Vancouver, British Columbia, Canada

D Smith, University of California, Los Angeles, Los Angeles, California

M Pallavicini, University of California, Merced, Merced, California

S Warren, Massachusetts General Hospital East and Harvard University School of Medicine,

Charlestown, Massachusetts

MM Sarwal, Stanford University, Stanford, California

DG Camp, RD Smith, W Qian, JM Jacobs, T Liu, F Yang, V Petyuk, BD Thrall, DL Stenoien, TC Squier, N Colton, BO Petritis, S Chowdhury, X Sun, AT Wright, I Marginean, X Zhang, KE Burnum, M

Hossain, ZM Tian, H Zhang, EW Robinson, LM Smith, JF Alfaro, NJ Jones, N Agrawal, Pacific

Northwest National Laboratory, Richland, Washington

N Sonenberg, McGill University, Montreal, Quebec, Canada

L Pasa-Tolic, R Zhao, HM Brewer, Environmental Molecular Sciences Laboratory, Richland,

Washington

MG Katze, J Cuschieri, University of Washington, Seattle, Washington

MH Ginsberg, RL Klemke, University of California, San Diego, La Jolla, California

JS Kim, Seoul National University, Seoul, Korea South, Republic Of

S Lee, J Lee, H Jung, Korea University, Seoul, Korea South, Republic Of

J Nelson, Oregon Health Sciences University/Oregon Graduate Institute, Portland, Oregon

T Kohwi-shigematsu, Lawrence Berkeley National Laboratory, Berkeley, California

A Umar, Erasmus University Medical Center, Rotterdam, Rotterdam, Netherlands

JA Mannick, JA Lederer, Harvard Medical School, Boston, Massachusetts

RN Kulkarni, Joslin Diabetes Center, Boston, Massachusetts

Y Wang, PNNL, Richland, Washington

\section{Combinatorial Operando Catalyst Research}

D Hu, ZC Zhang, G Xia, G Li, DL King, VM Lebarbier, Pacific Northwest National Laboratory, Richland, Washington

\section{Combined Castor Marker and Isotope Profile for Ricin Forensics}

HW Kreuzer, DS Wunschel, JJ Moran, HA Colburn, Pacific Northwest National Laboratory, Richland, Washington

MK Newburn, Environmental Molecular Sciences Laboratory, Richland, Washington 


\section{Combined NMR/Optical Microscopy of Biofilm Physiology Studies}

R Lux, University of California, Los Angeles, Los Angeles, California

PD Majors, Pacific Northwest National Laboratory, Richland, Washington

JS McLean, J. Craig Venter Institute, San Diego, California

Combined Theoretical and Experimental Studies of Laser Desorption from Metal Oxide Surfaces PV Sushko, London, University College, London, United Kingdom

AG Joly, WP Hess, Pacific Northwest National Laboratory, Richland, Washington

N Govind, K Kowalski, Environmental Molecular Sciences Laboratory, Richland, Washington

Combining In-Situ Buffer-Layer-Assisted-Growth with Scanning Probe Microscopy for Formation and Study of Supported Model Catalysts

I Lyubinetsky, Environmental Molecular Sciences Laboratory, Richland, Washington

Community Proteomic Analysis of a Near-Shore Anoxic Basin: Saanich Inlet

SJ Hallam, University of British Columbia, Vancouver, British Columbia, Canada

AD Norbeck, Pacific Northwest National Laboratory, Richland, Washington

Community-Based Biosignatures of Exposure and Functional Response in the Sediment-Water Interface of the Hyporheic Zone and Periphyton Community in River Systems

J Small, AL Bunn, Pacific Northwest National Laboratory, Richland, Washington

\section{Comparative Proteomic Analysis of Naturally Occurring Anaerobic Methane Oxidizing} Communities

SJ Hallam, University of British Columbia, Vancouver, British Columbia, Canada

AD Norbeck, Pacific Northwest National Laboratory, Richland, Washington

\section{Complex Enzymatic Reactions}

TP Straatsma, TA Soares Da Silva, DO Apiyo, Pacific Northwest National Laboratory, Richland, Washington

H Michel, E Herzog, Max Planck Institute of Biophysics, Frankfurt am Main, Germany

DE Gomes, Universidade Federal do Rio de Janeiro, Rio de Janeiro, Brazil

VH Helms, W Gu, Universitat des Saarlandes, Saarbrucken, Germany

M Elstner, Technical University Braunschweig, Braunschweig, Germany

Complex Processes in Separations, Catalysis, Hybrid Materials, and Nuclear Energy and Waste Management

Y Houndonougbo, Eastern Washington University, Cheney, Washington

AE Clark, Washington State University, Pulman, Washington

R Devanathan, F Gao, Pacific Northwest National Laboratory, Richland, Washington

MF Salvetti, NE Miller, HJ Kulik, N Bonini, N Marzari, Massachusetts Institute of Technology,

Cambridge, Massachusetts

PA Moreira, Universidade Estadual De Campinas, Sao Paulo, Brazil

LR Corrales, EE Moore, University of Arizona, Tucson, Arizona

R Zhang, Rensselaer Polytechnic Institute, Troy, New York

EJ Bylaska, Environmental Molecular Sciences Laboratory, Richland, Washington

K Tsemekhman, University of Washington, Seattle, Washington 
J Du, University of North Texas, Denton, Texas

D Chen, Hunan University, Hunan, China

Composition Analysis of Surface-Bound Nanoparticle Monolayers

ML Jespersen, JE Hutchison, JA Dahl, University of Oregon, Eugene, Oregon

Comprehensive Analysis Of Post-Translational Modifications (PTMs) In Proteins Involved In Histone Pre-mRNA Processing By High Performance nanoLC-MS

H Zhang, Pacific Northwest National Laboratory, Richland, Washington

R Thapar, University of North Carolina, Buffalo, New York

Computational and Experimental Investigations on NMR Properties of Actinide Compounds

J Li, HM Cho, SD Burton, Pacific Northwest National Laboratory, Richland, Washington

\section{Computational Chemical Dynamics of Complex Systems}

J Gao, CJ Cramer, DG Truhlar, JI Siepmann, DM York, EE Dahlke, P Jaque, ZH Li, R Valero Montero, A Sorkin, O Tishchenko, Y Zhao, JD Scanlon, K Wong, W Xie, Y Zhou, A Cembran, S Ma, JL Rafferty, K Nam, AN Jay, BJ Lynch, KE Anderson, EA Amin, J Zheng, BA Ellingson, Y Fan, A Marenich, RM Olson, S Mielke, HR Leverentz, LJ Fiedler, B Wang, E Papajak, M Mantina, K Yang, B White, BB Averkiev, University of Minnesota, Minneapolis, Minnesota SM Kathmann, M Dupuis, GK Schenter, B Ginovska, V Glezakou, CJ Mundy, P Fan, Pacific Northwest National Laboratory, Richland, Washington

MB Tsai, Brookhaven National Laboratory, Upton, New York

A Heyden, University of South Carolina, Columbia, South Carolina

M Valiev, K Kowalski, Environmental Molecular Sciences Laboratory, Richland, Washington

MA Iron, Weizmann Institute, Rehovot, Israel

A Chakraborty, Pennsylvania State University, University Park, Pennsylvania

MA Cunningham, The University of Texas-Pan American, Edinburg, Texas

\section{Computational Design of Catalysts: The Control of Chemical Transformation to Minimize the Environmental Impact of Chemical Processes}

JM Caruthers, KA Novstrup, JM Switzer, Purdue University, West Lafayette, Indiana

MA Barteau, AC Lukaski, University of Delaware, Newark, Delaware

Q Ge, L Cheng, Southern Illinois University, Carbondale, Illinois

A Altun, I Kerkines-Keramidas, AE Kuznetsov, Emory University, Atlanta, Georgia

K Morokuma, Kyoto University, Kyoto, Japan

RL Abel, Columbia University, New York, New York

DM Camaioni, J Li, D Mei, J Franz, M Dupuis, R Rousseau, Pacific Northwest National Laboratory,

Richland, Washington

F Mehmood, Argonne National Laboratory, Argonne, Illinois

WF Schneider, RB Getman, University of Notre Dame, Notre Dame, Indiana

CR Landis, E Mavrikakis, LC Grabow, PA Ferrin, RP Nabar, AU Nilekar, FM Eichhorn, G Peng,

University of Wisconsin-Madison, Madison, Wisconsin

JF Haw, DM McCann, University of Southern California, Los Angeles, California

M Neurock, VM Vadhri, C Buda, CP Plaisance, Y Cai, University of Virginia, Charlottesville, Virginia

DA Dixon, S Li, M Hernandez Matus, MT Nguyen, DJ Grant, R Craciun, T Wang, VE Jackson, JE Dyer,

EB Garner, M Chen, AC Stott, M Vasiliu, University of Alabama, Tuscaloosa, Tuscaloosa, Alabama 
$\mathrm{L} \mathrm{Xu,} \mathrm{University} \mathrm{of} \mathrm{Texas} \mathrm{at} \mathrm{Austin,} \mathrm{Austin,} \mathrm{Texas}$

$\mathrm{J} \mathrm{Du}$, University of North Texas, Denton, Texas

MJ Janik, Pennsylvania State University, University Park, Pennsylvania

MS Gutowski, S Ling, AV Abramov, ZG Keolopile, Heriot-Watt University, Edinburgh, United

Kingdom

DC Ford, J Jiao, JA Herron, CA Farberow, JH Stotz, University of Wisconsin, Madison, Madison,

Wisconsin

L Wang, Washington State University Tri-Cities, Richland, Washington

MS Kelley, University of Alabama, Tuscaloosa, Alabama

NA Deskins, Worcester Polytechnic Institute, Worcester, Massachusetts

\section{Computational Design of Materials for Hydrogen Storage}

P Jena, Q Wang, Q Sun, S Li, K Boggavarapu, Virginia Commonwealth University, Richmond, Virginia CE Strickland, Washington State University, Pullman, Washington

Z Zhang, Stanford University, Stanford, California

J Li, JE Jaffe, SM Kathmann, GK Schenter, CJ Mundy, Pacific Northwest National Laboratory, Richland, Washington

A Nikitin, Stanford Linear Accelerator Center, Menlo Park, California

PJ Hay, N Henson, Los Alamos National Laboratory, Los Alamos, New Mexico

W Stier, L Arnadottir, A Arnaldsson, K Tsemekhman, University of Washington, Seattle, Washington

DA Dixon, DJ Grant, JR Batson, M Hernandez Matus, MT Nguyen, University of Alabama, Tuscaloosa,

Tuscaloosa, Alabama

GA Henkelman, University of Texas at Austin, Austin, Texas

H Jonsson, University of Iceland, Reykjavík, Iceland

RA Bachorz, Universitaet Karlsruhe, Karlsruhe, Germany

MS Gutowski, A Whiteside, RG McKinlay, AV Abramov, Heriot-Watt University, Edinburgh, United

Kingdom

\section{Computational Investigation of Metal Clusters on Doped Carbon Supports}

CH Turner, CK Acharya, W An, University of Alabama, Tuscaloosa, Tuscaloosa, Alabama

\section{Computational Rheology of Dense Suspensions}

A Chatterjee, DR Heine, Corning, Inc., Corning, New York

Computational Studies of the Heterogeneous Production of Molecular Chlorine by OH Oxidation of Chloride in Sea Salt

DJ Tobias, R D'auria, University of California, Irvine, Irvine, California

SS Xantheas, CJ Mundy, Pacific Northwest National Laboratory, Richland, Washington

\section{Computational Study of Protein-Protein Interaction Dynamics at Single Molecule Level} JN Wang, Q Lu, State University of New York at Stony Brook, Stony Brook, New York HP Lu, Y He, J Cao, SP Rajapaksha, Bowling Green State University, Bowling Green, Ohio

\section{Conformational Dynamics of Pin1 Regulation of APP processing and Abeta Production} LK Nicholson, Cornell University, Ithaca, New York 
Contribution of Biosorption Processes to the Sustained Removal of Uranium from Groundwater in the Absence of Added Electron Donor

LA N'guessan, DR Lovley, University of Massachusetts Amherst, Amherst, Massachusetts

Coprecipitation of Aluminum Hydroxides and Calcium Carbonates during Denitrification: A Stabilization Strategy for Oxidized Metals and Radionuclides

JD Istok, Oregon State University, Corvallis, Oregon

M Oostrom, MA Covert, Pacific Northwest National Laboratory, Richland, Washington

TW Wietsma, Environmental Molecular Sciences Laboratory, Richland, Washington

Correlating the Active Site Structure of Copper Metalloproteins With Their Spectroscopic and Chemical Properties

AS Lipton, Pacific Northwest National Laboratory, Richland, Washington

GS Harbison, University of Nebraska, Lincoln, Nebraska

Correlation of Structure and Function of Zinc Metalloproteins Via a Combined NMR/Molecular Theory Approach

G Parkin, Columbia University, New York, New York

AS Lipton, PD Ellis, R Heck, TP Straatsma, P Moore, Pacific Northwest National Laboratory, Richland, Washington

CA Fierke, M Hernick, J Penner-hahn, University of Michigan, Ann Arbor, Michigan

M Valiev, Environmental Molecular Sciences Laboratory, Richland, Washington

Correlation of Structure and Function of Zinc Metalloproteins Via a Combined NMR/Molecular Theory Approach

ER Kantrowitz, Boston College, Chestnut Hill, Massachusetts

AS Lipton, PD Ellis, Pacific Northwest National Laboratory, Richland, Washington

CA Fierke, M Hernick, University of Michigan, Ann Arbor, Michigan

M Valiev, Environmental Molecular Sciences Laboratory, Richland, Washington

Coupled Hydrological and Geochemical Studies on Uranium Plumes at Hanford Site: Reactions, Spatial Distributions, Speciation, and Future Mobility

Z Wang, LJ Poindexter, Pacific Northwest National Laboratory, Richland, Washington

Coupling Experiments with Theory: Effect of Size and Topography on Structural Dynamics and Energetic Reactions of Metal Oxides

N Govind, Environmental Molecular Sciences Laboratory, Richland, Washington

S Stankic, CNRS, Paris, France

Coupling Temporal Variations in Geochemistry to Activities of Microbial Populations in the Deep Subseafloor Biosphere

JE Turse, SJ Callister, CD Nicora, Pacific Northwest National Laboratory, Richland, Washington

PR Girguis, JC Robidart, Harvard University, Cambridge, Massachusetts

Course in Nanoscience and Nanotechnology

S Gupta, Portland State University, Portland, Oregon

RD Souza, Y Qiang, C Berven, DS Choi, University of Idaho, Moscow, Idaho 
A Datta, GC Eastland, X Ye, L Zhang, AR Smith, Washington State University, Pullman, Washington N Karri, Pacific Northwest National Laboratory, Richland, Washington

DR Baer, Environmental Molecular Sciences Laboratory, Richland, Washington

F Ohuchi, University of Washington, Seattle, Washington

MV Peeler, Washington State Department of Ecology, Olympia, Washington

L Wang, Washington State University Tri-Cities, Richland, Washington

Cryo-EM Investigations of Interfacial Bacterial Extracellular Polymeric Substance (EPS) in Vanadium Interactions

MJ Marshall, Pacific Northwest National Laboratory, Richland, Washington

Cryogenic XPS Study of Metal Oxide/Water Interfaces

J Boily, Umea University, Umea, Sweden

Cryo-TEM Investigation of Polyketide Synthase Megacomplexes in Candidatus Endobugula Sertula and Teredinibacter Turnerae

A Dohnalkova, Environmental Molecular Sciences Laboratory, Richland, Washington

MG Haygood, AE Silva, Oregon Health Sciences University/Oregon Graduate Institute, Beaverton, Oregon

Cyanobacteria Membrane Biology Grand Challenge: Systems Analysis of the Dynamics of Membrane Architecture, Composition, and Function- Proteomic, Metabolomic, and Metallomic Characterization

JM Jacobs, DW Koppenaal, TO Metz, Q Zhang, Pacific Northwest National Laboratory, Richland, Washington

HB Pakrasi, J Stockel, Washington University in St. Louis, St. Louis, Missouri

Defects and Defect Processes in Ceramics

WJ Weber, W Jiang, I Bae, BD Milbrath, Pacific Northwest National Laboratory, Richland, Washington LA Boatner, Oak Ridge National Laboratory, Oak Ridge, Tennessee

X Xiang, University of Michigan, Ann Arbor, Michigan

R Linares, Universidade de Sao Paulo, Sao Paulo, Brazil

Y Zhang, Environmental Molecular Sciences Laboratory, Richland, Washington

PD Edmondson, PNNL, Richland, Washington

Degradation of Lignin Using Homogeneous Catalysis in Ionic Liquids

G Li, SD Burton, ZC Zhang, AS Lea, JE Amonette, Pacific Northwest National Laboratory, Richland, Washington

JM White, University of Texas at Austin, Austin, Texas

Demonstrate a Systematic Application of a Magnetic Tweezers: Holding a Protein Molecule in Solution

HP Lu, Bowling Green State University, Bowling Green, Ohio

Dependence of High Affinity of Metalloprotease for Inhibitor on Internal Millisecond Motions

SR Van Doren, University of Missouri, Columbia, Columbia, Missouri 


\section{Desiccation of Porous Media to Limit Contaminant Migration}

M Oostrom, MA Covert, G Tartakovsky, Pacific Northwest National Laboratory, Richland, Washington JH Dane, Auburn University, Auburn, Alabama

TW Wietsma, Environmental Molecular Sciences Laboratory, Richland, Washington

M Sinha, JM Dohman, AW Easterday, PNNL, Richland, Washington

Design Criteria for Organic Charge Transporting Materials through Computational Modeling Ls Sapochak, AB Padmaperuma, PE Burrows, Pacific Northwest National Laboratory, Richland, Washington

M Valiev, N Govind, Environmental Molecular Sciences Laboratory, Richland, Washington

Design of Micro-Cantilever Actuators for Measuring Surface Tension of Sub Microliter Volumes Applied Towards Monitoring Interfacial Processes

DW Britt, BA Henrie, Utah State University, Logan, Utah

Detection of Gas Inclusion in Organic And Metal-Organic Solids

P Thallapally, ca fernandez, RK Motkuri, Pacific Northwest National Laboratory, Richland, Washington

Determination Li Concentration in Wide Band Gap SnO2 Semiconductor, Using RBS Technique SB Rananavare, A Chaparadza, Portland State University, Portland, Oregon

Determination of Cr and O Diffusion Coefficients in (Co,Mn)3O4 Coatings for IT-SOFC Steel Interconnects

RJ Smith, Montana State University, Bozeman, Montana

B Arey, C Wang, Environmental Molecular Sciences Laboratory, Richland, Washington

Determination of Enhanced ZnO Nucleation and Growth by Modeling, Simulation and MOCVD Under UV Irradiation

VT Woods, B Luttrell, G Huebner, Peak Materials, Colorado Springs, Colorado

Determination of Saturation-Capillary Pressure Relations of Crude Oil-Brine Systems to Estimate Oil-Water Interfacial Areas

L Zhong, Pacific Northwest National Laboratory, Richland, Washington

Determination of the CCR5 and CXCR4 Antagonist and Agonist Binding Site.

EA Dratz, T Liu, Montana State University, Bozeman, Montana

Determine 3d-Structure of Chemokine in Apo Form and in Complex With Its Native Receptor by Solid State NMR

AS Lipton, Pacific Northwest National Laboratory, Richland, Washington

CM Rienstra, University of Illinois at Urbana-Champaign, Urbana, Illinois

L Mueller, Bristol-Myers Squibb, Princeton, New Jersey 
Determining Mechanisms of Nonlinear Photo Response and Transport Properties in Scintillating Materials

F Gao, TC Droubay, TC Kaspar, Pacific Northwest National Laboratory, Richland, Washington

Y Zhang, V Shutthanandan, L Saraf, Environmental Molecular Sciences Laboratory, Richland,

Washington

W Zhang, Peking University, Beijing, China

Determining the Effects of Organic Chemical Mixtures and Wastewater on DNAPL Migration H Yoon, AJ Valocchi, C Werth, University of Illinois, Urbana, Illinois

M Oostrom, MA Covert, Pacific Northwest National Laboratory, Richland, Washington

TW Wietsma, Environmental Molecular Sciences Laboratory, Richland, Washington

Develop Fundamental Surface Science Characterization Data for Use in Improving Mixed Alcohol Synthesis Catalysts

JF White, SM Kathmann, D Mei, R Rousseau, Pacific Northwest National Laboratory, Richland,

Washington

Develop Fundamental Surface Science Characterization Data for Use in Improving Mixed Alcohol Synthesis Catalysts

SM Kathmann, MA Gerber, JF White, R Rousseau, D Mei, V Glezakou, Pacific Northwest National

Laboratory, Richland, Washington

C Wang, MH Engelhard, A Andersen, Environmental Molecular Sciences Laboratory, Richland,

Washington

G Murdachaew, PNNL, Richland, Washington

Developing a Systems Biology Approach for the Termite Gut Microbial Isolate Verrucomicrobium Sp. Strain TAV2

JL Rodrigues, The University of Texas at Arlington, Arlington, Texas

Developing an in Situ Assay for Determining Spatial and Temporal Autoinducer Concentrations in Wound Biofilms

KP Rumbaugh, Texas Tech University, Lubbock, Texas

Developing Calculation Methods to Precisely Measure Proteome Proteins and Their PostTranslational Modifications

SO Purvine, Y Shen, Pacific Northwest National Laboratory, Richland, Washington

N Tolic, KK Hixson, Environmental Molecular Sciences Laboratory, Richland, Washington

Developing in-Spectrometer Photochemistry and Integration of Molecular Imaging Techniques:

Probing Photoinduced Electronic Structure Variations in Dye-Sensitized TiO2 Model System

MA Henderson, Pacific Northwest National Laboratory, Richland, Washington

A Pandey, Michigan Technological University, Houghton, Michigan

BA Parkinson, Colorado State University, Fort Collins, Colorado

DR Baer, T Thevuthasan, KM Beck, Z Zhu, P Nachimuthu, Environmental Molecular Sciences

Laboratory, Richland, Washington

S Puvanendran, Western Michigan University, Kalamazoo, Michigan 
Development and Applications of Novel Approaches for Analysis of Organic Aerosols using Highresolution Mass Spectrometry.

MN Treiger, University of Pennsylvania, Philadelphia, Pennsylvania

A Laskin, Environmental Molecular Sciences Laboratory, Richland, Washington

$\mathrm{X}$ Yang, H Chen, Fudan University, Shanghai, China

Development of 1st Principles Dynamics With Exact Exchange and Spin-Orbit ZORA

EJ Bylaska, M Valiev, WA De Jong, Environmental Molecular Sciences Laboratory, Richland, Washington

JH Weare, University of California, San Diego, La Jolla, California

SA Bogatko, Université Libre de Bruxelles, Brussels, Belgium

R Atta-Fynn, D Johnson, PNNL, Richland, Washington

Development of a Microfluidics Flow and Transport Laboratory

C Werth, University of Illinois, Urbana, Illinois

M Oostrom, MA Covert, Pacific Northwest National Laboratory, Richland, Washington

TW Wietsma, Environmental Molecular Sciences Laboratory, Richland, Washington

C Zhang, PNNL, Richland, Washington

Development of Accurate Force Fields for Aqueous Biological Environments Using a Massively Parallel Multiscale Approach

KF Freed, B Roux, LR Scott, University of Chicago, Chicago, Illinois

SS Xantheas, S Yoo, Pacific Northwest National Laboratory, Richland, Washington

JR Hammond, J Bardhan, Argonne National Laboratory, Argonne, Illinois

K Kowalski, M Valiev, Environmental Molecular Sciences Laboratory, Richland, Washington

WA Farone, Applied Power Concepts, Inc., Anaheim, California

Development of Alternative and Durable High Performance Cathode Supports for PEM Fuel Cells

P Kumar, Washington State University, Pullman, Washington

Y Wang, J Liu, J Kwak, Z Zhang, VV Viswanathan, Y Shao, R Kou, Pacific Northwest National

Laboratory, Richland, Washington

MH Engelhard, Environmental Molecular Sciences Laboratory, Richland, Washington

Development of an Ultra-High Throughput and Highly Sensitive Proteomics Instrumental Platform YM Ibrahim, E Baker, GA Anderson, K Tang, ME Belov, RD Smith, RT Kurulugama, TR Clauss, Pacific Northwest National Laboratory, Richland, Washington

Development of High Throughput Proteomic Production Operations (PNNL Scope \# 40601, Dick Smith's OBER Proteomics)

N Colton, RD Smith, EW Robinson, E Baker, EA Livesay, YM Ibrahim, H Kang, NM Lourette, JS Page, AK Shukla, Pacific Northwest National Laboratory, Richland, Washington

L Pasa-Tolic, Environmental Molecular Sciences Laboratory, Richland, Washington

C Costello, Boston University, Boston, Massachusetts

Q Luo, Northeastern Universiy, Boston, Maine 
Development of Live and LC-NMR Microbial Metabolomics Methods for Systems Biology Studies: A Test Case Relevant to BioFuels Production

PD Majors, HD Hamilton, Pacific Northwest National Laboratory, Richland, Washington

NG Isern, Environmental Molecular Sciences Laboratory, Richland, Washington

BK Ahring, Bioproducts, Sciences, and Engineering Laboratory, BSEL, Richland, Washington

Development of Novel Approaches for Analysis of Secondary Organic Aerosols Using High-

Resolution Mass Spectrometry.

S Nizkorodov, AP Bateman, DL Bones, University of California, Irvine, Irivne, California

J Laskin, Pacific Northwest National Laboratory, Richland, Washington

MN Treiger, University of Pennsylvania, Philadelphia, Pennsylvania

A Laskin, Environmental Molecular Sciences Laboratory, Richland, Washington

AL Chang-Graham, Amherst College, Amherst, Massachusetts

Development of Novel Electrolyte and Electrode Materials for a New Generation of Low-

Temperature SOFCs Using First-Principles Atomistic Thermodynamics

M Liu, Y Choi, Georgia Institute of Technology, Georgia Tech Research Corporation, Atlanta, Georgia

Development of O-18 Isotope Ratio Measurements of Uranium Oxides and Surface Metal Oxides for Forensic Analysis

HW Kreuzer, AJ Carman, DC Duckworth, JJ Moran, Pacific Northwest National Laboratory, Richland, Washington

MK Newburn, Environmental Molecular Sciences Laboratory, Richland, Washington

Development of Room Temperature Ferromagnetism in Wide-band-gap Oxide Semiconductor Nanostructures

A Punnoose, AP Thurber, Boise State University, BOISE, Idaho

Development of the Novel Hybrid Nanowire-based Solar Cell on a Flexible Substrate

DS Choi, University of Idaho, Moscow, Idaho

Development of Time-Dependent Quantum Reaction Dynamics for Surface Reactions

JR Rustad, University of California, Davis, Davis, California

GK Schenter, Pacific Northwest National Laboratory, Richland, Washington

EJ Bylaska, D Wang, N Govind, Environmental Molecular Sciences Laboratory, Richland, Washington

Development of Tunable Highly Efficient THz Sources using Focused Ion Beam (FIB)-Based

Lithography

SK Sundaram, BJ Riley, JV Crum, Pacific Northwest National Laboratory, Richland, Washington

L Saraf, Environmental Molecular Sciences Laboratory, Richland, Washington

Development of Tunable THz Sources using Focused Ion Beam (FIB)-Based Lithography

SK Sundaram, P Keller, H Qiao, Pacific Northwest National Laboratory, Richland, Washington

L Saraf, Environmental Molecular Sciences Laboratory, Richland, Washington 
Diffusion Measurements in Polymers and Their Composites

LV Smith, Washington State University, Pullman, Washington

SP Pilli, Pacific Northwest National Laboratory, Richland, Washington

Discovery Novel Therapeutic Protein Targets by Advanced MS Proteomics

W Qian, RD Smith, Pacific Northwest National Laboratory, Richland, Washington

J Zhou, PNNL, Richland, Washington

Discovery of Biomarkers Related to COPD

SM Varnum, J Pounds, Pacific Northwest National Laboratory, Richland, Washington

Distance-Dependent Electron Transfer to Mineral Surfaces Mediated by Bacterial Multiheme Cytochromes

NS Wigginton, MF Hochella, Virginia Polytechnic Institute, Blacksburg, Virginia

KM Rosso, Pacific Northwest National Laboratory, Richland, Washington

DNA Methylation-Dependent Patterns of Histone Post-Translational Modification

CS Pikaard, Washington University in St. Louis, Saint Louis, Missouri

DNAPL-Water Interfacial Area Determinations During Entrapped DNAPL Dissolution Using Tracer Techniques

S Bottero, Utrecht University, Utrecht, Italy

M Schroth, Swiss Federal Institute of Technology, Zurich, Switzerland

Doping Inorganic Wide-Bandgap Semiconductors for Future Energy Devices

W Jiang, Pacific Northwest National Laboratory, Richland, Washington

Dynamic Changes in Molecular Interactions along the Circadian Rhythm (Orr-LDRD Membrane Biology GC, PNNL Scope \#90001)

G Orr, DJ Panther, KJ Cassens, Pacific Northwest National Laboratory, Richland, Washington

HB Pakrasi, Washington University in St. Louis, St. Louis, Missouri

T Ogawa, Shanghai Institute of Plant Physiology and Ecology, Shanghai, China

Dynamic Characterization of the Enzyme Phosphomannomutase/Phosphoglucomutase From P. Aeruginosa

SR Van Doren, LJ Beamer, University of Missouri, Columbia, Columbia, Missouri

Early Transition Metal Oxides as Catalysts: Crossing Scales from Clusters to Single Crystals to Functioning Materials

E Iglesia, University of California, Berkeley, Berkeley, California

CH Peden, J Hu, J Kwak, J Liu, Y Wang, X She, G Xia, D Kim, JF White, VM Lebarbier, L Zhang,

Pacific Northwest National Laboratory, Richland, Washington

$\mathrm{B} \mathrm{Xu}, \mathrm{Y} \mathrm{Wu}$, Tsinghua University, Beijing, China

MH Engelhard, Environmental Molecular Sciences Laboratory, Richland, Washington

DA Dixon, University of Alabama, Tuscaloosa, Tuscaloosa, Alabama

S Cho, Chonnam National University, Guangju, Korea South, Republic Of 
K Lee, Korea University, Seoul, Korea South, Republic Of

J Jeon, Kongju National University, Gongju, Korea South, Republic Of

Edgar Buck’s Proposal

EC Buck, RN Wittman, Pacific Northwest National Laboratory, Richland, Washington

Effect of Changing Rainfall and Temperature on Biochemical Composition of Soil Organic Matter JF Banfield, University of California, Berkeley, Berkeley, California

AA Berhe, University of California, Merced, Merced, California

MP Waldrop, US Geological Survey, Menlo Park, Menlo Park, California

Effect of Porosity and Surface Finish on Whisker Growth and Morphology

U Sahaym, Washington State University, Pullman, Washington

Effect of Structure and Impurities on Charge Transfer Reactions in Solid State Electrochemical Devices

LR Pederson, Pacific Northwest National Laboratory, Richland, Washington

S Gopalan, UB Pal, SN Basu, L Miara, KF Ludwig, KE Smith, JN Davis, Boston University, Brookline, Massachusetts

Effects of Nitrogen Additions on Soil Carbon Pools in Two Humid Tropical Forest Types

DF Cusack, University of California, Berkeley, Berkeley, California

MS Torn, Lawrence Berkeley National Laboratory, Berkeley, California

Effects of Surfaces on Photogenerated Electron Diffusion in Zinc Oxide Nanowires

Y Gu, Washington State University, Pullman, Washington

Electrochemical Analyses of the Electron Transfer Rates of MtrC and OmcA to Hematite

L Shi, SN Jiang, Y Xiong, Pacific Northwest National Laboratory, Richland, Washington

A Dohnalkova, Environmental Molecular Sciences Laboratory, Richland, Washington

SM Belchik, PNNL, Richland, Washington

Electron and Hole Trapping and Excitations in Polycrystalline Oxides: Challenge for Materials Simulations

AL Shlyuger, KP McKenna, London, University College, London, United Kingdom

MJ Wolf, University College London, London, United Kingdom

Electron Microscopy Imaging of Cyanobacteria Synechcystis Sp.

C Gassman, Columbia Basin College, Pasco, Washington

A Dohnalkova, B Arey, Environmental Molecular Sciences Laboratory, Richland, Washington

Electron Valence Characteristics of Rare-Earth Ions in Wide Bandgap Semiconductor Nanowires Y Gu, P Dhakal, JK Dhal, Washington State University, Pullman, Washington

Elucidating the Degree of Deuteration in Synthetic Triuranyl Diphosphate Tetrahydrate (TDT) Using Raman Confocal Microscopy

CR Armstrong, SB Clark, Washington State University, Pullman, Washington 
Enabling Quantitatively Predictive Understanding of Multifluid Flow and Multicomponent Biogeochemical Reactive Transport in Complex, Subsurface Systems

AJ Valocchi, University of Illinois, Urbana-Champaign, Illinois

SB Yabusaki, DH Bacon, VL Freedman, AM Tartakovsky, ML Rockhold, Y Fang, M Oostrom, F Zhang, AL Ward, G Lin, Pacific Northwest National Laboratory, Richland, Washington

PC Lichtner, Los Alamos National Laboratory, Los Alamos, New Mexico

JC Parker, F Zhang, Oak Ridge National Laboratory, Oak Ridge, Tennessee

M White, Mississippi State University, Mississippi State, Mississippi

M Rosing, Self-Employed (Rosing), Ft. Collins, Colorado

RJ Versteeg, idaho national laboratory, Idaho Falls, Idaho

Energetics of Non-Covalent Interactions in Biological Systems

A Woods, National Institute of Health, Baltimore, Maryland

Z Yang, University of Colorado Boulder, Boulder, Colorado

Energetics, Dynamics and Mechanisms of Dissociation of Peptide Radical Cations

IK Chu, N Lam, T Song, The University of Hong Kong, Pokfulam, Hong Kong

C Siu, York University, Toronto, Ontario, Canada

Z Yang, University of Colorado Boulder, Boulder, Colorado

Energy and Entropy Effects in Dissociation of Non-Covalent Complexes: A Combined

Experimental and Theoretical Investigation

J Laskin, Pacific Northwest National Laboratory, Richland, Washington

Z Yang, University of Colorado Boulder, Boulder, Colorado

$\mathrm{X}$ Shi, JH Parks, Rowland Institute at Harvard, Cambridge, Massachusetts

\section{Energy Smart Data Center}

A Adhikari, Portland State University, Portland, Oregon

A Marquez, Pacific Northwest National Laboratory, Richland, Washington

Enhanced Ionic Conductivity of Samaria Doped Ceria Thin Films Through Tailoring the Dopant Concentration and Microstructures

Z Yu, Nanjing Normal University, Nanjing, China

Environmental Proteomic Analysis of Anaerobic Methane Oxidizing Systems

L Constan, SJ Hallam, University of British Columbia, Vancouver, British Columbia, Canada

Epitaxial Growth and Properties of Magnetically Doped ZnO Prepared by Pulsed Laser Deposition of Nanoparticle Targets

MJ Hopkins, Portland State University, Portland, Oregon

DR Dixon, Washington State University, Pullman, Washington

SA Chambers, TC Droubay, TC Kaspar, Pacific Northwest National Laboratory, Richland, Washington

C Wang, Environmental Molecular Sciences Laboratory, Richland, Washington

KM Whitaker, CR Johnson, KR Kittilstved, DR Gamelin, SA Santangelo, PI Archer, Y Li, ST

Ochsenbein, B Zhao, University of Washington, Seattle, Washington 
Equilibrium-Induced Decomposition of Nitrates on a Nox Storage/Reduction Catalyst

WS Epling, University of Waterloo, Waterloo, Ontario, Canada

Evaluate and Apply the LC/FAIMS/IMS/TOF MS Platform to High Throughput Quantitative Analysis

K Tang, E Baker, R Mabrouki, WF Danielson, Pacific Northwest National Laboratory, Richland, Washington

Examination of NanoC60 Aggregates Through Dialysis Membranes As Surrogates for Cell Membrane Diffusion

AS Felsot, KL Stump, Washington State University Tri-Cities, West Richland, Washington

Experimental and Theoretical Investigations on the Chemistry and Dynamics of Ammonia Borane Dehydrogenation

MR Hartman, Oregon State University, Corvallis, Oregon

D Neiner, University of California, Davis, Davis, California

TJ Johnson, T Autrey, NJ Hess, WJ Shaw, A Karkamkar, J Li, J Linehan, CJ Mundy, SM Kathmann, DM

Camaioni, RG Potter, AT Luedtke, CM Sorensen, EC Ronnebro, Pacific Northwest National Laboratory,

Richland, Washington

CW Hamilton, H Kim, TE Proffen, Los Alamos National Laboratory, Los Alamos, New Mexico

MO Jones, University of Oxford, Oxford, United Kingdom

LR Alden, DW Himmelberger, University of Pennsylvania, Philadelphia, Pennsylvania

Y Chua, National University of Singapore, Singapore, Singapore

MH Engelhard, Environmental Molecular Sciences Laboratory, Richland, Washington

ME Bowden, Industrial Research Ltd., Lower Hutt, New Zealand

G Severa, University of Hawaii Manoa, Honolulu, Hawaii

WI David, Rutherford Appleton Laboratory, Chilton, Oxfordshire, United Kingdom

Experimental and Theoretical Studies of Reaction Pathways for the Breakdown of Chlorinated Hydrocarbon Molecules by Metal and Metal Oxide Nanoparticles(including PNNL Scope \# 42184/44076, Baer/Amonette/Tratnyek Nano-Fe BES/EMSP project)

J Antony, Y Qiang, A Sharma, H Che, MK Singh, H Han, University of Idaho, Moscow, Idaho

RL Penn, C Chun, University of Minnesota, Minneapolis, New Mexico

M Dupuis, BD Kay, JE Amonette, Z Dohnalek, GS Parkinson, DW Matson, J Liu, Pacific Northwest

National Laboratory, Richland, Washington

AA El-azab, Florida State University, Tallahassee, Florida

EJ Bylaska, R Kukkadapu, DR Baer, MH Engelhard, TW Wietsma, C Wang, S Kuchibhatla, PL

Gassman, Environmental Molecular Sciences Laboratory, Richland, Washington

PG Tratnyek, J Nurmi, V Sarathy, P Lee, Oregon Health Sciences University/Oregon Graduate Institute,

Beaverton, Oregon

S Suzer, Bilkent University, Ankara, Turkey

Experimental Investigation and Theoretical Validation of Growth, Morphology, and Chemistry of Cuprous Oxide Nanoclusters on STO (100) Substrate

CH Henager, SK Sundaram, Pacific Northwest National Laboratory, Richland, Washington

S Kuchibhatla, T Thevuthasan, Environmental Molecular Sciences Laboratory, Richland, Washington

Z Yu, Nanjing Normal University, Nanjing, China 
Experimental Investigation of Sr-90 Subsurface Contamination Sequestration in Hanford 100N Area Sediments by Surface Infiltration of an Apatite Solution JE Szecsody, M Oostrom, MA Covert, Pacific Northwest National Laboratory, Richland, Washington TW Wietsma, Environmental Molecular Sciences Laboratory, Richland, Washington

Experimental Investigation of Strontium Mass Transfer in a Dynamic Vadose Zone - Aquifer River System

SB Yabusaki, M Oostrom, MA Covert, Pacific Northwest National Laboratory, Richland, Washington EJ Bylaska, TW Wietsma, Environmental Molecular Sciences Laboratory, Richland, Washington

Experimental Studies of Fundamental Molecule-Particle Interactions and Reactions on Iron Metal and Oxide Surfaces

RS Smith, BD Kay, Z Dohnalek, GS Parkinson, Pacific Northwest National Laboratory, Richland, Washington

Exploring the Boundaries of Metazoan Thermotolerance at Hydrothermal Vents: Respiration and Protein Expression of Paralvinellid Worms

SJ Callister, Pacific Northwest National Laboratory, Richland, Washington

PR Girguis, GF Dilly, Harvard University, Cambridge, Massachusetts

Exploring the Mechanisms and Kinetics of Small Peptides Towards the Search for the Global Minimum Through Molecular Dynamics Simulations

D Shemesh, EW Schlag, Technische Universität München, Garching, Germany

Exploring the Proteome of E. coli Following Adaptation to Environmental and Genetic

Perturbation

BO Palsson, University of California, San Diego, La Jolla, California

Fabrication and Characterization of High-Performance Transparent Transistor

F Hua, Clarkson University, Potsdam, New York

Fabrication of a Carbon Nanotube Thermionic Cathode

KJ Bunch, H Qiao, Pacific Northwest National Laboratory, Richland, Washington

PL Gassman, Environmental Molecular Sciences Laboratory, Richland, Washington

Facility-Wide Management and Storage for Scientific Data

KJ Auberry, Environmental Molecular Sciences Laboratory, Richland, Washington

FAST MAS probe Technology for 900 MHz NMR

CH Peden, J Kwak, J Hu, Pacific Northwest National Laboratory, Richland, Washington

Fate and Transport of Titanium Dioxide Through Freshwater Ecosystems

DJ Gaspar, A Miracle, AL Bunn, JA Ward, Pacific Northwest National Laboratory, Richland,

Washington

Federally-Funded Fellows Program in EMSL for 2009

TJ Law, Environmental Molecular Sciences Laboratory, Richland, Washington

JL Coffen, Self (Coffen), Richland, Washington 
DA Harper, Amelia L. Johnson High School, Thomaston, Alabama

DK Belcher, North Pines Middle School, Spokane Valley, Washington

Ferromagnetic Study of ZnO Based Heterojunctions

N Athavan, R Könenkamp, Portland State University, Portland, Oregon

Ferromagnetism in MgO by Nitrogen Doping

C Yang, IBM Almaden Research Center, San Jose, California

Ferromagnetism in MgO by Nitrogen Doping

SD Conradson, Los Alamos National Laboratory, Los Alamos, New Mexico

C Yang, IBM Almaden Research Center, San Jose, California

FIB/SEM/EBSD/EDS Study of the Grain boundary Character Effect on Precipitation

L Tan, AG Certain, TR Allen, University of Wisconsin-Madison, Madison, Wisconsin

Y Yang, University of Washington, Seattle, Washington

Fine-Tuning Ambipolar Charge-Transport Properties of Organic Materials By Computational Design

AB Padmaperuma, Ls Sapochak, J Rainbolt, Pacific Northwest National Laboratory, Richland, Washington

N Govind, M Valiev, Environmental Molecular Sciences Laboratory, Richland, Washington

First Principles Adaptive Kinetic Monte Carlo Calculations of Catalytic Reactions at Supported Nanoparticles

D Mei, Pacific Northwest National Laboratory, Richland, Washington

GA Henkelman, L Xu, RC Terrell, University of Texas at Austin, Austin, Texas

First Principles Multiscale Analysis of Biochemical Processes: Signal Transduction and Spectroscopic Analysis of Local Structure-Folding Relations in Membrane Proteins

MB Luskin, University of Minnesota, Minneapolis, Minnesota

JH Miller, Washington State University, Richland, Washington

PA Cole, Johns Hopkins University, Baltimore, Maryland

TP Straatsma, Pacific Northwest National Laboratory, Richland, Washington

BM Elsasser, University of Paderborn, Paderborn, Germany

JC Mitchell, University of Wisconsin-Madison, Madison, Wisconsin

EJ Bylaska, K Kowalski, M Valiev, Environmental Molecular Sciences Laboratory, Richland,

Washington

W E, Princeton University, Princeton, New Jersey

JH Weare, J Adams, SB Baden, JE Kim, PW Langhoff, JA Mccammon, SS Taylor, W Wang, SE Wong,

P Cheng, RP Daly, University of California, San Diego, La Jolla, California

EL Cauet, Université Libre de Bruxelles, Brussels, Belgium

Flow and Transport of Edible Oil Emulsions for Enhanced Bioremediation

MJ Truex, Pacific Northwest National Laboratory, Richland, Washington 
Flow Battery Research

G Xia, C Huang, S Kim, Pacific Northwest National Laboratory, Richland, Washington

Fluid Spreading on Micro-Patterned Surfaces

MI Dragila, Oregon State University, Corvalis, Oregon

Fluorescence Characteristics of Polyvinyltoluene Scintilator

DM Strachan, AG Joly, Pacific Northwest National Laboratory, Richland, Washington

SC Gott, AD Crosby, Walla Walla College, Walla Walla, Washington

Q Ma, Walla Walla University, Walla Walla, Washington

Foam Delivery of Calcium Polysulfide for Cr(VI) Remediation in Unsaturated Porous Media - 2-D Flow Cell Experimental Study

L Zhong, Pacific Northwest National Laboratory, Richland, Washington

Formation of Apatite in Hanford Vadose Zone Sediments by Gaseous Injection of Phosphorus Compounds

ML Rockhold, JE Szecsody, J Mckinley, Pacific Northwest National Laboratory, Richland, Washington

Fourier Transform Spectrum of the Iodine Cell Used for Exoplanet Searches at the Lick Observatory

DA Fischer, San Francisco State University, San Francisco, California

Free radical Reactions in the Catalytic Cycle of Cytochrome bc Complexes

DM Kramer, JL Cape, IP Forquer, Washington State University, Pullman, Washington

TP Straatsma, Pacific Northwest National Laboratory, Richland, Washington

M Valiev, Environmental Molecular Sciences Laboratory, Richland, Washington

MK Bowman, University of Alabama, Tuscaloosa, Tuscaloosa, Alabama

FRET-Based Molecular Dynamics Simulation of Membrane-Receptor Activation

G Orr, L Opresko, KJ Cassens, SM Bing, Pacific Northwest National Laboratory, Richland, Washington K Wittrup, Massachusetts Institute of Technology, Cambridge, Massachusetts

HS Wiley, ER Vorpagel, Environmental Molecular Sciences Laboratory, Richland, Washington

JA Balch, PNNL, Richland, Washington

From Gas Phase Clusters to Nanomaterials

L Wang, W Huang, L Cui, H Zhai, Z Sun, X Xing, Washington State University, Richland, Washington C Ning, Tsinghua University, Beijing, China

PD Dau, Brown University, Providence, Rhode Island

S Li, Xinzhou Teachers University, Xinzhou City, Shanxi, China

L Wang, Washington State University Tri-Cities, Richland, Washington

G Xiao, Xi' an Jiaotong University, Xi'an, Shaanxi, China

FTS Scan of Keck Iodine Cell

GW Marcy, University of California, Berkeley, Berkeley, California

DA Fischer, San Francisco State University, San Francisco, California 


\section{Functional Materials for Separation and Sensing}

JT Bays, S Addleman, JW Grate, MG Warner, W Yantasee, CL Warner, LS Fifield, JW Pittman, J

Davidson, R Rutledge, Pacific Northwest National Laboratory, Richland, Washington

B Arey, MH Engelhard, A Dohnalkova, Environmental Molecular Sciences Laboratory, Richland,

Washington

Fundamental Investigations of Heterogeneous Catalysis Using Isotopic Transient Kinetic Analysis

S Chuang, University of Akron, Akron, Ohio

RS Disselkamp, VM Lebarbier, CH Peden, Pacific Northwest National Laboratory, Richland,

Washington

M White, Mississippi State University, Mississippi State, Mississippi

CT Campbell, Y Yang, L Cameron, IM Jensen, University of Washington, Seattle, Washington

J Goodwin, Clemson University, Clemson, South Carolina

C Mims, University of Toronto, Toronto, Ontario, Canada

\section{Fundamental Mechanistic Studies of Catalytic Hydrogen Production and Utilization}

CH Peden, J Szanyi, D Mei, Pacific Northwest National Laboratory, Richland, Washington

H Aleksandrov, University of Sofia, Sofia, Bulgaria

Y Yang, CT Campbell, University of Washington, Seattle, Washington

GA Henkelman, RC Terrell, University of Texas at Austin, Austin, Texas

C Mims, University of Toronto, Toronto, Ontario, Canada

\section{Fundamental Studies of Heterogeneous Photocatalysis on Model TiO2 Surfaces}

MA Henderson, R Zehr, Pacific Northwest National Laboratory, Richland, Washington

Y Du, I Lyubinetsky, Environmental Molecular Sciences Laboratory, Richland, Washington

\section{Fundamental Studies of Nitrogen Oxide Surface chemistry: A Model System Approach}

Y Kim, Hanbat National University (formerly Taejon National University of Technology), Taejon, Korea South, Republic Of

J Szanyi, CH Peden, D Mei, Pacific Northwest National Laboratory, Richland, Washington

C Yi, Texas A\&M University, College Station, Texas

I Lyubinetsky, Environmental Molecular Sciences Laboratory, Richland, Washington

GA Henkelman, RC Terrell, University of Texas at Austin, Austin, Texas

F Netzer, Karl-Franzens-University Graz, Graz, Austria

Fundamental Studies of Nitrogen Oxide Surface Chemistry: A Model System Approach Combining Experiments and Theory

K Mudiyanselage, J Szanyi, J Kwak, D Mei, Pacific Northwest National Laboratory, Richland, Washington

RC Terrell, University of Texas at Austin, Austin, Texas

Fundamental Studies on Biomolecule Ions Desorbed from Open-Air Surfaces

O Hadjar, Pacific Northwest National Laboratory, Richland, Washington

FM Fernandez, L Nyadong, Georgia Institute of Technology, Georgia Tech Research Corporation, Atlanta, Georgia

Z Yang, University of Colorado Boulder, Boulder, Colorado 
Fundamental Understanding of Charge Transfer From a Ru-Based Dye Adsorbate to a TiO2(110) Surface

MA Henderson, Pacific Northwest National Laboratory, Richland, Washington

BA Parkinson, Colorado State University, Fort Collins, Colorado

T Thevuthasan, P Nachimuthu, Environmental Molecular Sciences Laboratory, Richland, Washington

\section{Genomes-to-Life Protein Complex Isolation Capability}

BS Hooker, K Victry, D Auberry, C Lin, Pacific Northwest National Laboratory, Richland, Washington HS Wiley, Environmental Molecular Sciences Laboratory, Richland, Washington

Global Cloud Resolving Model: Benchmarking, Data Access, and Model Testing

KL Schuchardt, BJ Palmer, AS Koontz, J Piernas Canovas, JA Daily, Pacific Northwest National

Laboratory, Richland, Washington

RJ Latham, Argonne National Laboratory, Argonne, Illinois

RP Heikes, DA Randall, DA Dazlich, Colorado State University, Fort Collins, Colorado

Global Proteomic Analysis of Human Ehrlichiosis Agents

Y Rikihisa, Ohio State University, Columbus, Ohio

AD Norbeck, Pacific Northwest National Laboratory, Richland, Washington

Glycolipid Structure, Dynamics and Recognition in a Membrane Environment

RJ Woods, ML Demarco, MB Tessier, BY Austin, CR Daniels, University of Georgia, Athens, Georgia

Grand Challenge in Membrane Biology

LA Sherman, T Summerfield, Purdue University, West Lafayette, Indiana

GW Buchko, JM Jacobs, Pacific Northwest National Laboratory, Richland, Washington

HB Pakrasi, BK Ghosh, Washington University in St. Louis, St. Louis, Missouri

R Kunnavakkam, R Aurora, Saint Louis University, St. Louis, Missouri

N Koropatkin

Donald Danforth Plant Science Center, St. Louis, Missouri

T Ogawa, Shanghai Institute of Plant Physiology and Ecology, Shanghai, China

\section{Great Lakes Bioenergy Research Center}

MS Lipton, Pacific Northwest National Laboratory, Richland, Washington

\section{Gregory Szulczewski's Proposal}

GJ Szulczewski, University of Alabama, Tuscaloosa, Tuscaloosa, Alabama

\section{Growth Mechanisms of Graphene on Thin Ni Films}

J Kong, Massachusetts Institute of Technology, Cambridge, Massachusetts

\section{Growth Media Component Peptide and Carbohydrate Analysis}

KL Wahl, DS Wunschel, Pacific Northwest National Laboratory, Richland, Washington

\section{Growth-Phase Regulation of the Proteome of Rhodobacter Capsulatus}

SJ Callister, Pacific Northwest National Laboratory, Richland, Washington

AS Lang, Memorial University of Newfoundland, St. John's, Newfoundland, Canada 


\section{Heart Metabolomics During Ventricular Unloading}

OM Hyyti, University of Washington, Seattle, Washington

MA Portman, A Olson, Children's Hospital and Regional Medical Center, Seattle, Washington

Heterogeneous Ice Nucleation from Biomass Burning Particles: The Effect of Chemical Composition and Chemical Aging

A Laskin, C Wang, PL Gassman, Environmental Molecular Sciences Laboratory, Richland, Washington DA Knopf, BN Wang, State University of New York at Stony Brook, Stony Brook, New York

\section{Heterogeneous Photochemistry of Organic-Coated Mineral Dust Aerosol}

VH Grassian, MA Young, OB Laskina, University of Iowa, Iowa City, Iowa

A Laskin, Environmental Molecular Sciences Laboratory, Richland, Washington

\section{Heterogeneous Uptake of HNO3 on CaCO3: Determination of the Gas-Particle Reaction}

Probability as Function of Relative Humidity

VH Grassian, ER Gibson, OB Laskina, University of Iowa, Iowa City, Iowa

JP Cain, H Wang, University of Southern California, Los Angeles, California

A Laskin, Environmental Molecular Sciences Laboratory, Richland, Washington

Y Liu, University of California, San Diego, La Jolla, California

High Accuracy Modeling of Frequency Dependent Polarizabilities: Exploring the Cutting Edge Limits of NWChem

PJ Nichols, Pacific Northwest National Laboratory, Richland, Washington

JR Hammond, Argonne National Laboratory, Argonne, Illinois

WA De Jong, K Kowalski, M Valiev, D Wang, N Govind, KR Glaesemann, EJ Bylaska, Environmental

Molecular Sciences Laboratory, Richland, Washington

High Performance Sequence Analysis for Data-Intensive Bioinformatics

CS Oehmen, L Mccue, Pacific Northwest National Laboratory, Richland, Washington

HJ Sofia, Whitman College, Walla Walla, Washington

EA Welsh, Washington University in St. Louis, St. Louis, Missouri

DJ Baxter, Environmental Molecular Sciences Laboratory, Richland, Washington

High Pressure VLS Synthesis of Silicon Nanowires

SB Rananavare, JC Chan, Portland State University, Portland, Oregon

High Resolution 1H NMR Metabolomics Using Slow and Ultra Slow Magic Angle Spinning Q Zhang, TO Metz, J Hu, KR Minard, M Murphy, K Thrall, A Woodstock, Pacific Northwest National Laboratory, Richland, Washington

D Rommereim, J Sears, Environmental Molecular Sciences Laboratory, Richland, Washington

J Caceres-cortes, Bristol-Myers Squibb, Lawrenceville, New Jersey

High Resolution Electron Microscopy Study of Nanostructured Materials

J Liu, K Zhu, dn wang, Z Nie, R Kou, D Choi, J Zhang, Pacific Northwest National Laboratory, Richland, Washington

D Liu, University of Washington, Seattle, Washington 
High Resolution Fourier Transform Infrared Spectroscopy Studies of Multidimensional H Transfer in Tropolone/Tropolonoid Molecules-a Bridge to the Behavior in Biological Systems

TA Blake, RL Sams, Pacific Northwest National Laboratory, Richland, Washington

RL Redington, Texas Tech University, Lubbock, Texas

High Resolution Infrared Spectroscopy of Spiropentane and Bicyclopentane

JW Nibler, RW Kirkpatrick, MA Martin, AJ Perry, JE Price, Oregon State University, Corvallis, Oregon A Weber, National Institute of Standards and Technology, Gaithersburg, Maryland

AG Maki, Private (Maki), Mill Creek, Washington

A Masiello, California State University East Bay, Hayward, California

High Resolution Mass Spectrometry of High Molecular Weight Compounds Formed Via the Heterogeneous Reaction of $\mathrm{OH}$ with Simple Organic Aerosols

K Wilson, J Smith, Lawrence Berkeley National Laboratory, University of California, Berkeley, Berkeley, California

A Laskin, C Wang, Environmental Molecular Sciences Laboratory, Richland, Washington

High Resolution TEM of Doped Ceria System

S Omar, JC Nino, University of Florida, Gainesville, Florida

High Sensitivity Atomic Absorption Flux Sensing and Control for Next-Generation Oxide Molecular Beam Epitaxy in EMSL

SA Chambers, Pacific Northwest National Laboratory, Richland, Washington

E Friedman, k-Space Associates, Inc., Dexter, Michigan

High Temperature, Large Sample Volume, Constant Flow Magic Angle Spinning NMR Probe for 11.7 T Magnetic Field for In-situ Catalytic Reaction Characterization

$\mathrm{J} \mathrm{Hu}$, Pacific Northwest National Laboratory, Richland, Washington

JJ Ford, J Sears, Environmental Molecular Sciences Laboratory, Richland, Washington

High Throughput Proteomic \& Metabolomic Early Biomarkers (PNNL Scope \#47174, RD Smith's EIF project)

T Liu, RD Smith, DG Camp, MA Gritsenko, Pacific Northwest National Laboratory, Richland,

Washington

High-k Oxides and Their Mixed Oxide Systems

CV Ramana, University of Texas at El Paso, El Paso, Texas

Highly Accurate Atomistic Quantum Chemical to Coarse Grained Study of DNA and RNA P Hobza, Academy of Sciences of the Czech Republic, Prague 6, Czech Republic

High-Performance Computing to Evaluate Hierarchical Heterogeneity Paradigms in Sedimentary Aquifer Systems

TD Scheibe, VL Freedman, Pacific Northwest National Laboratory, Richland, Washington

RW Ritzi, R Ramanathan, A Guin, Wright State University, Dayton, Ohio 
High-Resolution 27Al and 133Cs NMR of Weathered Hanford Sediments

KT Mueller, CR Strepka, C Fleeger, WJ Brouwer, CA Ryan, Pennsylvania State University, University Park, Pennsylvania

High-Resolution Interfacial Studies of Nano-Scale Oxide Films

S Ramanathan, C Chang, M Tsuchiya, Harvard University, Cambridge, Massachusetts

High-Resolution Magic Angle Spinning NMR Spectroscopy of Microtubule-Associated Motor Proteins

Y Han, TE Polenova, S Sun, S Paramasivam, University of Delaware, Newark, Delaware

High-Throughput Evaluation of Breast Cancer Markers

RC Zangar, Pacific Northwest National Laboratory, Richland, Washington

High-Throughput Proteomics: Applications and Development (PNNL Scope \#40601 and \#43488)

JD Ryan, J Shutthanandan, A Kalyanaraman, Washington State University, Pullman, Washington

SB Levy, Tufts University School of Medicine, Boston, Massachusetts

CS Oehmen, GA Anderson, MS Lipton, ME Monroe, A Polpitiya, AR Shah, RD Smith, DJ Anderson, JN

Adkins, C Ansong, E Baker, ME Belov, JN Brown, SJ Callister, TR Clauss, N Colton, WF Danielson,

MA Gritsenko, T Heibeck, YM Ibrahim, JM Jacobs, RT Kelly, DW Koppenaal, T Liu, D Lopez-Ferrer, I

Marginean, TO Metz, RJ Moore, CD Nicora, AD Norbeck, DJ Orton, JS Page, BO Petritis, K Petritis, V

Petyuk, S Prasad, DC Prior, W Qian, NJ Jones, EW Robinson, AA Schepmoes, Y Shen, AK Shukla, K

Tang, AV Tolmachev, JE Turse, KK Weitz, AT Wright, F Yang, Q Zhang, M Luna, RA Heegel, CN

Thiel, E Zink, X Zhang, Pacific Northwest National Laboratory, Richland, Washington

JR Stanley, Texas A\&M University, College Station, Texas

BL Lamarche, L Pasa-Tolic, KK Hixson, HM Brewer, R Zhang, R Zhao, Environmental Molecular

Sciences Laboratory, Richland, Washington

J Zhou, PNNL, Richland, Washington

HIV Proteomic Center for Host-Viral Response Characterization

JM Jacobs, RD Smith, TE Angel, Pacific Northwest National Laboratory, Richland, Washington

EY Chan, University of Washington, Seattle, Washington

Host Cell Interactions with Human Infectious Diseases, Straub

K Honer Zu Bentrup, Tulane University, New Orleans, Louisiana

TM Straub, CO Valdez, R Bartholomew, CJ Bruckner-Lea, Pacific Northwest National Laboratory, Richland, Washington

CP Gerba, P Orosz Coglhan, University of Arizona, Tucson, Arizona

A Dohnalkova, B Arey, Environmental Molecular Sciences Laboratory, Richland, Washington

C Nickerson, Arizona State University, Tempe, Arizona

J Vinje, Center for Disease Control Prevention, Atlanta, Georgia

S Fout, Environmental Protection Agency, Cincinnati, Ohio

Hybrid Numerical Methods for Multiscale Modeling of Subsurface Biogeochemical Processes G Lin, BJ Palmer, TD Scheibe, Y Fang, AM Tartakovsky, GE Hammond, L Sun, GD Black, JM Chase, KL Schuchardt, TO Elsethagen, VL Freedman, HE Trease, JA Fort, WA Perkins, H Silva, JA Daily, CL Rakowski, Pacific Northwest National Laboratory, Richland, Washington 
BA Allan, Sandia National Laboratory, Livermore, California

V Gurumoorthi, PNNL, Richland, Washington

\section{Hydrogen Bonding in Inhomogeneous Environments}

SI Sukharev, AG Anishkin, University of Maryland, College Park, Maryland

ME Green, VS Znamenskiy, S Liao, City College of New York, New York, New York

M Mezei, Mount Sinai School of Medicine, New York, New York

Hydrogen Materials Compatibility Studies

CH Henager, JD Holbery, KJ Alvine, Pacific Northwest National Laboratory, Richland, Washington

Hydrogen Production-(Steam Reforming of Hydrocarbons Including Methane, Propane, Gasoline, Jet Fuel and Biomass Derived Products)

J Hu, RA Dagle, D Howe, B Roberts, CM Fischer, G Whyatt, VM Lebarbier, A Karim, J Sun, Pacific

Northwest National Laboratory, Richland, Washington

MH Engelhard, B Arey, Environmental Molecular Sciences Laboratory, Richland, Washington

AB Kazi, KL Durand, AV Lyles, University of Arkansas at Pine Bluff, Pine Bluff, Arkansas

$\mathrm{S}$ Lu, Beijing Research Institute of Chemical Industry, Beijing, China

Hygroscopic Properties of Aerosol Organics

A Zelenyuk, R Zaveri, J Yang, T Vaden, Pacific Northwest National Laboratory, Richland, Washington

AG Hallar, DH Lowenthal, Desert Research Institute, Reno, Nevada

Identification and Validation of Protein Profiles Associated With Therapy-Resistance in Breast

Cancer

L Pasa-Tolic, Environmental Molecular Sciences Laboratory, Richland, Washington

A Umar, TM Luider, JA Foekens, Erasmus University Medical Center, Rotterdam, Rotterdam,

Netherlands

Identification of Precipitates and Chemistry of Interfaces in Optical Materials

DF Bahr, A Bellou, MM Reynolds, Washington State University, Pullman, Washington

Identify Morphology of Initial LIGO Core Optics Scatter Sites:

CA Vorvick, G Billingsley, California Institute of Technology, Pasadena, California

Identifying Targets for Therapeutic Interventions using Proteomic Technology

JN Adkins, KD Rodland, N Manes, S Chowdhury, JN Brown, RN Brown, C Ansong, EW Robinson,

MC Burnet, Pacific Northwest National Laboratory, Richland, Washington

HM Brewer, Environmental Molecular Sciences Laboratory, Richland, Washington

F Heffron, S Wong, R Estep, Oregon Health Sciences University/Oregon Graduate Institute, Portland, Oregon

Imaging of a Solid Oxide Fuel Cell Cathode

EM Ryan, C Amon, Carnegie Mellon University, Pittsburgh, Pennsylvania

Imaging Respiratory Structure and Function In Laboratory Animals

KR Minard, RA Corley, C Timchalk, R Jacob, Pacific Northwest National Laboratory, Richland,

Washington 
Immiscibility Features Between Silica-Rich and Carbonate-Rich Material in Breccia From the Sierra Madera Impact Structure.

SA Huson, MC Pope, Washington State University, Pullman, Washington

Impurity Segregation and Precipitation in Semiconductor Nanostructures

ST Dunham, BC Trzynadlowski, W Jiang, L Lin, H Lai, R Chen, University of Washington, Seattle, Washington

In Situ Nuclear Magnetic Resonance Investigations of Trapping Mechanisms in $\mathrm{CO}_{2}$ Storage J Hu, J Kwak, Pacific Northwest National Laboratory, Richland, Washington

DW Hoyt, J Sears, Environmental Molecular Sciences Laboratory, Richland, Washington

Inelastic Electron Scattering Cross Section Measurements in Liquid Water

NG Petrik, RG Tonkyn, GA Kimmel, Pacific Northwest National Laboratory, Richland, Washington

CM Brown, Wadsworth Center, Albany, New York

Infiltration, Redistribution, and Dissolution of Ethanol-Blended Gasoline: Intermediate-Scale Flow Cell Experimentation to Develop a Data Base for Numerical Model Testing and Verification.

T Ginn, University of California, Davis, Davis, California

Infrared Reflectance of Solids

TA Blake, TJ Johnson, Pacific Northwest National Laboratory, Richland, Washington

Infrared Reflectance Spectroscopy of Soils (Blake’s NNSA, PNNL Scope \#43865)

TA Blake, Pacific Northwest National Laboratory, Richland, Washington

PL Gassman, Environmental Molecular Sciences Laboratory, Richland, Washington

In-Situ Electron Microscopy and Spectroscopy Studies of Interfaces in Advanced Li-Ion Batteries Under Dynamic Operation Conditions

P Plachinda, Portland State University, Portland, Oregon

Z Yang, J Liu, Pacific Northwest National Laboratory, Richland, Washington

A Prasad, Michigan Technological University, Houghton, Michigan

T Thevuthasan, C Wang, B Arey, Environmental Molecular Sciences Laboratory, Richland, Washington

Instrument Testing and Deployment for the DOE CHAPS Field Campaign

CM Berkowitz, R Zaveri, Pacific Northwest National Laboratory, Richland, Washington

L Alexander, Environmental Molecular Sciences Laboratory, Richland, Washington

JA Ogren, National Oceanic and Atmospheric Administration (NOAA), Boulder, Colorado

Integrate The So-Called CRENBL Spin-Orbit Operator Into NWChem

WC Ermler, Self (Ermler), San Antonio, Texas

Integrated Experimental and Modeling Studies on Aerosol Evolution and Aging

R Zaveri, RC Easter, A Zelenyuk, J Yang, C Song, T Vaden, Pacific Northwest National Laboratory,

Richland, Washington

L Alexander, Environmental Molecular Sciences Laboratory, Richland, Washington 
Integrated Experimental and Modeling Studies on Secondary Organic Aerosol Formation

R Zaveri, SM Kathmann, A Zelenyuk, DA Maughan, JC Birnbaum, J Yang, C Song, T Vaden, Pacific

Northwest National Laboratory, Richland, Washington

S Madronich, National Center for Atmospheric Research, boulder, Colorado

A Laskin, Environmental Molecular Sciences Laboratory, Richland, Washington

JA Thornton, University of Washington, Seattle, Washington

Integrated Top-Down and Bottom-up Proteomics Strategy to Identify Multisite and Multistep Modifications on Radiation Sensitive Proteins

DL Stenoien, Pacific Northwest National Laboratory, Richland, Washington

Interfaces and Interactions: Non-ideal Behavior of Biological Molecules

W Chiu, EL Zechiedrich, Baylor College of Medicine, Houston, Texas

BM Pettitt, SL Johnsson, R Glowinski, TE Warth, GC Lynch, K Wong, KM Dyer, C Chen, JS Perkyns, W Li, JJ Howard, GL Randall, CY Hu, J Feng, H Kokubo, JG Reid, B Lin, Y Bai, University of Houston, Houston, Texas

\section{Interfacial Excited State Dynamics on TiO2}

WP Hess, Pacific Northwest National Laboratory, Richland, Washington

K Tanimura, Osaka University, Osaka, Japan

KM Beck, Environmental Molecular Sciences Laboratory, Richland, Washington

Interfacial Phenomena in Cu Plating

VM Dubin, Y Tao, J Blanchard, X Xu, L Maretti, K Agapiou, eMAT Technology LLC, Moses Lake, Washington

Interrogation of Glucose Metabolism by Oral Biofilms Using Combined NMR/Optical Spectroscopy and Stable Isotope Labeling

JC Scholten, PD Majors, Pacific Northwest National Laboratory, Richland, Washington

JS McLean, J. Craig Venter Institute, San Diego, California

Introducing Parallel Computing to Undergraduate Students

TA Beck, Pacific Northwest National Laboratory, Richland, Washington

KA Glass, DM Brown, BL Lamarche, A Andersen, Environmental Molecular Sciences Laboratory,

Richland, Washington

SM Dixon, Washington State University Tri-Cities, Richland, Washington

FC Kwok, CG Simmons, LC Carlsen, TD Gibson, PNNL, Richland, Washington

Investigating Nox Effects on Soa Formation in the Western United States Using WRF-Chem

Regional Modeling

JL Fry, Reed College, Portland, Oregon

Investigating Post-Translational Modifications of Kinesin

SP Gross, University of California, Irvine, Irvine, California

Investigation of Peptide Fragmentation using Soft-Landing

S Yoon, VH Wysocki, University of Arizona, Tucson, Arizona 
Investigation of Water-Soluble X-ray Luminescence Nanoparticles for Biomedical Applications W Chen, The University of Texas at Arlington, Arlington, Texas

Investigations into Sulfur Poisoning Mechanisms in Solid-State Oxide Fuel Cells Using QuantumChemical Computations

M Liu, J Wang, Y Choi, Georgia Institute of Technology, Georgia Tech Research Corporation, Atlanta, Georgia

Investigations into the Protein-Mineral Interface

ES Taylor, S Lower, Ohio State University, Columbus, Ohio

Investigations of Electric Field Gradients and NMR Chemical Shifts of 87Sr in Mixed Calcium/Strontium Systems Using High Field NMR and Computational Chemistry

KT Mueller, ET Poweleit, WJ Brouwer, Pennsylvania State University, University Park, Pennsylvania

Ion Beam Analysis of Components for Solid Oxide Fuel Cells

RJ Smith, M Finsterbusch, Montana State University, Bozeman, Montana

CV Ramana, University of Texas at El Paso, El Paso, Texas

Ion Beam Implantation of Titanium Alloys to Improve Durability in Structural and Biomedical Applications

MP Carroll, Washington State University, Pullman, Washington

KO Findley, Colorado School of Mines, Golden, Colorado

Ion Beam Methods for the Synthesis, Modification, and Characterization of Radiation Detection Materials

LA Boatner, JS Neal, Oak Ridge National Laboratory, Oak Ridge, Tennessee

Ion-Beam-Induced Structural Changes in Compound Semiconductors Studied by Advanced Electron Microscopy

M Ishimaru, Osaka University, Ibaraki, Japan

Isoform-Specific Quantitative Proteomics Applying N-terminal Enrichment and informatics deconvolution

W Qian, BO Petritis, Pacific Northwest National Laboratory, Richland, Washington

Kinetic Study of Heterogeneous Reaction of Palladium Nanoparticles with $\mathrm{O} 2$ and $\mathrm{OH}$

H Wang, JP Cain, University of Southern California, Los Angeles, California

A Laskin, PL Gassman, C Wang, Environmental Molecular Sciences Laboratory, Richland, Washington

Kinetic Study of Heterogeneous Reaction of Soot with Reactive Oxidants

H Wang, JP Cain, University of Southern California, Los Angeles, California

C Wang, A Laskin, PL Gassman, MH Engelhard, Environmental Molecular Sciences Laboratory,

Richland, Washington

Y Liu, University of California, San Diego, La Jolla, California 
Laboratory Measurements of Secondary Organic Aerosol Formation from Semi-Volatile Organic Compounds

J Shilling, R Zaveri, C Song, DJ Cziczo, Pacific Northwest National Laboratory, Richland, Washington

Large-Scale Computational Modeling of the Chemical Behavior of Actinide Elements at Interfaces V Bryantsev, MS Diallo, California Institute of Technology, Washington DC, Wash DC

AE Clark, J Kuta, Washington State University, Pullman, Washington

J Li, V Glezakou, Pacific Northwest National Laboratory, Richland, Washington

BP Hay, Oak Ridge National Laboratory, Oak Ridge, Tennessee

L Visscher, Vrijie Universiteit/Scientific Computing and Modelling, The Netherlands, Netherlands

NJ Roesch, BN Martorell Masip, S Kruger, Technische Universität München, Garching, Germany

WA De Jong, EJ Bylaska, P Yang, Environmental Molecular Sciences Laboratory, Richland, Washington

TL Windus, GE Schoendorff, Iowa State University, Ames, Iowa

DA Dixon, University of Alabama, Tuscaloosa, Tuscaloosa, Alabama

Q Pan, University of Manitoba, Winnipeg, Manitoba, Canada

R Atta-Fynn, D Johnson, PNNL, Richland, Washington

\section{Laser Desorption from Metal Oxides}

PV Sushko, AL Shlyuger, London, University College, London, United Kingdom

WP Hess, AG Joly, Pacific Northwest National Laboratory, Richland, Washington

KM Beck, Environmental Molecular Sciences Laboratory, Richland, Washington

MJ Wolf, University College London, London, United Kingdom

Laser-Induced Time-Resolved Fluorescence Spectroscopic Characterization of Ce, Er Doped YAG (Yttrium Aluminium Garnet) Crystals

D Solodovnikov, KG Lynn, Washington State University, Pullman, Washington

LC-FTMS Analysis of Parathion, Dichlor, and TETS

CG Fraga, Pacific Northwest National Laboratory, Richland, Washington

Lignin and Depolymerised Lignin Characterization by High Performance Liquid Chormotography Mass Spectrometry (LCMS)

R Zhang, Environmental Molecular Sciences Laboratory, Richland, Washington

\section{Linking Microscopic to Macroscopic Adsorption/Desorption Behavior for Uranium on}

Environmental Materials

KM Rosso, SC Smith, ES Ilton, O Qafoku, J Friese, P Bachelor, Z Wang, Pacific Northwest National

Laboratory, Richland, Washington

B Arey, Environmental Molecular Sciences Laboratory, Richland, Washington

Local Structure in Zirconia-Based Nuclear Fuels and Transmutation Targets

I Farnan, University of Cambridge, Cambridge, United Kingdom

J Somers, European Commission Joint Research Centre, Karlsruhe, Germany, Germany 
Location of Probes in Membrane and Model Membrane Systems and Their Effects on Lipid Reorangization

MA Sedgwick, DC Crans, B Baruah, E Gaidamauskas, AM Trujillo, Colorado State University, Fort

Collins, Colorado

\section{Longitudinal Proteomic Characterization of Human Cerebrospinal Fluid}

DG Camp, SO Purvine, T Liu, Pacific Northwest National Laboratory, Richland, Washington

SE Schutzer, University of Medicine and Dentistry, New Jersey Medical School, Newark, New Jersey

Low Temperature (10K) 25Mg Solid-State NMR of DNA Repair Proteins and Their Complexes

PD Ellis, AS Lipton, R Heck, P Moore, Pacific Northwest National Laboratory, Richland, Washington

M Valiev, Environmental Molecular Sciences Laboratory, Richland, Washington

DM Wilson, National Institute on Aging, IRP, NIH, Baltimore, Maryland

SH Wilson, National Institute of Environmental Health Sciences, NIH, RTP, North Carolina

\section{Lustre Scalability Research}

EJ Felix, DM Brown, Environmental Molecular Sciences Laboratory, Richland, Washington

B Schroeder, University of Toronto, Toronto, Ontario, Canada

Lustre Scalability Research

EJ Felix, DM Brown, J Carr, Environmental Molecular Sciences Laboratory, Richland, Washington

Magnetic Resonance Microscopy of Ozone-Induced Lung Injury

KR Minard, RA Corley, R Jacob, Pacific Northwest National Laboratory, Richland, Washington

Mapping Structural Dynamics of the SRP During Co-Translational Protein Targeting

S Shan, VQ Lam, California Institute of Technology, Pasadena, California

Massive Contingency Analysis with High Performance Computing

Y Chen, Pacific Northwest National Laboratory, Richland, Washington

DJ Baxter, Environmental Molecular Sciences Laboratory, Richland, Washington

Z Huang, BK Kalahar, S Jin, PNNL, Richland, Washington

Material Interactions at Interfaces in Solid Oxide Fuel Cells

JS Hardy, VL Sprenkle, KD Meinhardt, J Kim, NL Canfield, JF Bonnett, Pacific Northwest National

Laboratory, Richland, Washington

Material Interface Optimization in ETA-Photovoltaics

TC Droubay, TC Kaspar, Pacific Northwest National Laboratory, Richland, Washington

V Shutthanandan, C Wang, Environmental Molecular Sciences Laboratory, Richland, Washington

Material Modification by Dense Electronic Excitations

M Toulemonde, CIMAP-GANIL, Caen cedex 5, France

S Moll, Centre de Spectrometrie Nucleaire et de Spectrometrie de Masse, Orsay, France 
MBE Growth and Properties of Model TiO2 Surfaces for Fundamental Studies of Heterogeneous Photocatalysis

SA Chambers, MA Henderson, T Ohsawa, DJ Kim, Pacific Northwest National Laboratory, Richland, Washington

C Wang, P Nachimuthu, Y Du, Environmental Molecular Sciences Laboratory, Richland, Washington R Shao, unknown, Washington

MBE Growth and Properties of N-doped TiO2 for Enhanced Visible Light Absorption and Water Splitting

SA Chambers, S Cheung, MA Henderson, T Ohsawa, DJ Kim, Pacific Northwest National Laboratory, Richland, Washington

SM Heald, Argonne National Laboratory, Argonne, Illinois

J Rodriguez, Brookhaven National Laboratory, Upton, New York

C Wang, P Nachimuthu, V Shutthanandan, Environmental Molecular Sciences Laboratory, Richland, Washington

CS Fadley, Lawrence Berkeley National Laboratory, Richland, West Virginia

A Mangham, PNNL, Richland, Washington

Measurement and Optimization of the Redox Properties of Enzymes Using Single-Molecule Imaging, Controlled Electrochemical Potential, and Nanoscale Confinement

EJ Ackerman, D Hu, C Lei, C Windisch, TC Squier, Pacific Northwest National Laboratory, Richland, Washington

Measurement of Saturation-Dependent Anisotropy in Hydraulic Conductivity of Hanford Vadose Zone Sediments

F Zhang, Pacific Northwest National Laboratory, Richland, Washington

Measuring Nanometer Distances in the Packaging RNA Using Double Electron-Electron Resonance PZ Qin, X Zhang, GG Grant, A Popova, University of Southern California, Los Angeles, California

MK Bowman, University of Alabama, Tuscaloosa, Tuscaloosa, Alabama

Mechanisms and Control of Biofilm Formation: Membrane Biofouling

S Chellam, AR Badireddy, University of Houston, Houston, Texas

Mechanisms of Laser and Electron Beam Modifications of Wide Bandgap Single Crystal Surfaces JT Dickinson, E Khan, Washington State University, PUllman, Washington

WP Hess, Pacific Northwest National Laboratory, Richland, Washington

Mechanisms of Nanocrystalline Uraninite Oxidation by Iron Minerals Under of Sulfate-Reducing Bacteria

R Sani, RN Gurram, VR Kunreddy, South Dakota School of Mines and Technology, Rapid City, South Dakota

J De, South Dakota State University, Rapid City, South Dakota

Mechanisms of Ubiquitin Transfer and Regulation

PS Brzovic, R Klevit, D Fox, DM Wenzel, CM Eakin, C Heikaus, University of Washington, Seattle, Washington 
Mechanistic Studies of Protein Allostery at the Atomistic Scale

J Chu, JB Brokaw, University of California, Berkeley, Berkeley, California

Mechanistic Understanding of Zeolite Deactivation Pathways

KG Rappe, JL Male, J Kwak, CH Peden, Pacific Northwest National Laboratory, Richland, Washington

RG Silver, JL Ratts, Caterpillar, Inc., Mossville, Illinois

C Wang, Environmental Molecular Sciences Laboratory, Richland, Washington

Membrane Rearrangements in Dengue Virus Infected Cells

RM Perera, RJ Kuhn, AS Jannasch, J Adamec, Purdue University, West Lafayette, Indiana

Metabolomics and Proteomics of Bacterial Signaling

OM Mcdougal, K Cornell, Boise State University, Boise, Idaho

MHC Presented Peptide Quantitation: Mass Spectrometry Methods Development

HS Smallwood, St. Jude Children's Research Hospital, Memphis, Tennessee

Michael Knoblauch's Proposal Molecular Structure and Interaction of Forisome Filaments

M Knoblauch, DR Froelich, Washington State University, Pullman, Washington

Microfabrication of Electrospray Emitter Arrays for Improved Sensitivity in Electrospray Ionization-Mass Spectrometry

RT Kelly, RD Smith, K Tang, X Sun, N Agrawal, Pacific Northwest National Laboratory, Richland, Washington

Microsampling Isotope Ratio Mass Spectrometer Development

R Zaveri, A Mendoza, RA Dagle, BR Johnson, HW Kreuzer, DJ Gaspar, Pacific Northwest National Laboratory, Richland, Washington

Microscale and Spectroscopic Investigations in Geochemical and Microbiological Systems BH Lower, Ohio State University, Columbus, Ohio

JM Zachara, J Mckinley, ES Ilton, KM Rosso, Z Wang, C Liu, M Oostrom, DA Moore, MA Covert, TE Queen, G Tartakovsky, Pacific Northwest National Laboratory, Richland, Washington

R Kukkadapu, A Dohnalkova, TW Wietsma, B Arey, PL Gassman, Environmental Molecular Sciences Laboratory, Richland, Washington

J Boily, Umea University, Umea, Sweden

M Sinha, PNNL, Richland, Washington

Microscale Metabolic, Redox and Abiotic Reactions in Hanford 300 Area Subsurface Sediments

RS Renslow, H Beyenal, B Cao, Washington State University, Pullman, Washington

PD Majors, JK Fredrickson, CL Bilskis, Pacific Northwest National Laboratory, Richland, Washington

JS McLean, J. Craig Venter Institute, San Diego, California

Microscopic Mass Transfer of $U$ and Tc in Subsurface Sediments

S Fendorf, Stanford University, Stanford, California

C Liu, Z Wang, SN Kerisit, Pacific Northwest National Laboratory, Richland, Washington

RP Ewing, Iowa State University, Ames, Iowa 
Microscopic Mass Transfer on U(VI) and Tc(VIII) in Subsurface sediments

C Liu, Z Wang, Pacific Northwest National Laboratory, Richland, Washington

RP Ewing, Iowa State University, Ames, Iowa

Microscopy Investigation on Nanostructured Electrodes

J Zhang, dn wang, G Xia, J Liu, W Xu, RE Williford, AS Lea, Pacific Northwest National Laboratory,

Richland, Washington

MH Engelhard, Environmental Molecular Sciences Laboratory, Richland, Washington

Microstructural Evolution During Warm Deformation of Magnesium

A Rohatgi, Pacific Northwest National Laboratory, Richland, Washington

KF Mattlin, PNNL, Richland, Washington

Miniaturized Two Sector Field Analyzer for Complex Electro-Spray Source Mixture Separation and Ion-CCD Visualization Prior to Micro-Array Simultaneous Deposition

SM Shill, O Hadjar, O.I. Analytical, Pelham, Alabama

WC Schnute, Dionex Corporation, Sunnyvale, California

Mitotic Regulation of the Nuclear Pore Complex Protein Interaction Network

SA Osmani, Ohio State University, Columbus, Ohio

R Zhao, Environmental Molecular Sciences Laboratory, Richland, Washington

Mixed Oxide Films for Gas Sensing and Photo-Catalytic Applications

KR Padmanabhan, Wayne State University, Detroit, Michigan

Model Oxide Defects With Vicinally Stepped NiO(100) Substrates

MA Langell, University of Nebraska, Lincoln, Nebraska

Y Du, I Lyubinetsky, Environmental Molecular Sciences Laboratory, Richland, Washington

Modeling Charge Transport and Interfacial Chemistry at the Nanoscale

A Kumar, University of Central Florida, Orlando, Florida

R Devanathan, J Yu, Pacific Northwest National Laboratory, Richland, Washington

PA Moreira, Universidade Estadual De Campinas, Sao Paulo, Brazil

AE Hugo, Gonzaga University, Spokane, Washington

H Xiao, PNNL, Richland, Washington

Modeling Defect- and Interface-Induced Rapid Decomposition of Molecular Materials

PV Sushko, AL Shlyuger, London, University College, London, United Kingdom

NA Zarkevich, University of Maryland, College Park, Maryland

N Govind, Environmental Molecular Sciences Laboratory, Richland, Washington

MM Kukla, University of Maryland, College Park, College Park, Maryland

Modeling Multiscale-Multiphase-Multicomponent Subsurface Reactive Flows using Advanced Computing

GE Hammond, BN Lee, Pacific Northwest National Laboratory, Richland, Washington

PC Lichtner, Los Alamos National Laboratory, Los Alamos, New Mexico

RT Mills, Oak Ridge National Laboratory, Oak Ridge, Tennessee 
Modeling of Polymer Membrane Nanostructure and Its Effect on Proton and Small Molecule Transport

R Devanathan, V Glezakou, M Dupuis, S Morrison-smith, K Artiga, RD Lins, Pacific Northwest National Laboratory, Richland, Washington

A Venkatnathan, Indian Institute of Science Education and Research, Pune, Pune, India

\section{Modeling Reactive Flows in Porous Media}

GE Hammond, BN Lee, Pacific Northwest National Laboratory, Richland, Washington

PC Lichtner, Los Alamos National Laboratory, Los Alamos, New Mexico

RT Mills, Oak Ridge National Laboratory, Oak Ridge, Tennessee

Modification and Characterization of Radiation Behaviour of Zircon (ZrSiO4)

M Zhang, University of Cambridge, Cambridge, United Kingdom

Molecular Analysis of Initiation Factor Complexes Contain eIF3

S Miyamoto, JW Hershey, University of California, Davis, Davis, California

Molecular Beam Scattering Measurements on Anatase TiO2(001) and Rutile TiO2(110)

U Burghaus, North Dakota State University, Fargo, North Dakota

Molecular Computational Studies In Geochemistry and Environmental Chemistry

JR Rustad, WH Casey, MC Wander, University of California, Davis, Davis, California

KM Rosso, SN Kerisit, GK Schenter, M Dupuis, PJ Nichols, J Yu, Pacific Northwest National

Laboratory, Richland, Washington

JM Murray, Florida State University, Tallahassee, Florida

EJ Bylaska, M Valiev, Environmental Molecular Sciences Laboratory, Richland, Washington

DA Dixon, VE Jackson, MT Nguyen, University of Alabama, Tuscaloosa, Tuscaloosa, Alabama

JH Weare, University of California, San Diego, La Jolla, California

SA Bogatko, EL Cauet, Université Libre de Bruxelles, Brussels, Belgium

R Atta-Fynn, D Johnson, PNNL, Richland, Washington

Molecular Dynamics Study of an Actin Septamer: Testing the Holmes Model and the Hydrophobic Plug Loop Hypothesis

GC Schatz, KM Barrett, MJ McCullagh, JT Paci, Northwestern University, Evanston, Illinois

KC Holmes, Max Planck Institut fur medizinische Forschung, Heidelberg, Germany

Molecular Interfaces to Single-Crystal Anatase Surfaces

RJ Hamers, University of Wisconsin-Madison, Madison, Wisconsin

Molecular Level Construction of Functional Surfaces

PL Gassman, Environmental Molecular Sciences Laboratory, Richland, Washington

SM Gorun, LA Lapok, New Jersey Institute of Technology, Newark, New Jersey

Molecular-level Mechanisms of Microbial Basalt Alteration

AS Templeton, University of Colorado, Boulder, Colorado

H Staudigel, University of California, San Diego, La Jolla, California

BM Tebo, Oregon Health Sciences University/Oregon Graduate Institute, Portland, Oregon 
Molecular-level Understanding of Transport and Optic Properties of Doped Oxide Nanoclusters Y Qiang, J Antony, M Faheem, A Sharma, H Che, RD Souza, H Han, University of Idaho, Moscow, Idaho

C Wang, Environmental Molecular Sciences Laboratory, Richland, Washington

Morphology, Composition and Surface Properties of Novel Functional Multimetallic and Core-shell Nanoparticles and Assemblies

C Zhong, PN Njoki, BN Wanjala, R Loukrakpam, B Fang, L Wang, State University of New York at Binghamton, Binghamton, New York

Mössbauer Analysis of Microbial Redox Transformations of Naturally Occurring Fe(III)-Bearing Phyllosilicates

EE Roden, University of Wisconsin, Madison, Wisconsin

ES Shelobolina, University of Wisconsin-Madison, Madison, Wisconsin

R Kukkadapu, Environmental Molecular Sciences Laboratory, Richland, Washington

Mossbauer and XPS Characterization of Fe-Based Fischer-Tropsch Catalysts

R Kukkadapu, Environmental Molecular Sciences Laboratory, Richland, Washington

V Subramani, Research Triangle Institute, Research Triangle Park, North Carolina

MRI of Chronic Obstructive Pulmonary Disease

KR Minard, C Timchalk, R Jacob, Pacific Northwest National Laboratory, Richland, Washington

KM Gideon, Battelle Columbus, Richland, Washington

Multidimensional High-Field Magic-Angle Spinning NMR of Membrane and Nanocrystalline Proteins

LJ Sperling, AJ Nieuwkoop, CM Rienstra, University of Illinois at Urbana-Champaign, Urbana, Illinois

Multidimensional High-Field Magic-Angle Spinning NMR of Membrane and Nanocrystalline Proteins

CM Rienstra, AJ Nieuwkoop, LJ Sperling, G Comellas, University of Illinois at Urbana-Champaign, Urbana, Illinois

L Mueller, Bristol-Myers Squibb, Princeton, New Jersey

Multifunctional Nanostructures for Nano-Biotechnology Applications

A Punnoose, MR Kongara, AP Thurber, Boise State University, BOISE, Idaho

Nano- and Atomic-Level Engineered Adsorbent, Catalyst, and Membrane Materials for Energy and Environmental Application

A Karim, W Liu, XS Li, Y Su, H Wan, J Zhang, Pacific Northwest National Laboratory, Richland, Washington

MH Engelhard, Environmental Molecular Sciences Laboratory, Richland, Washington

Nanoparticle-Cell Interactions and Intracellular Fate; Roles of Particle Surface Properties

BL Maddux, University of Oregon, Eugene, Oregon

KJ Cassens, G Orr, Pacific Northwest National Laboratory, Richland, Washington

GA Deitz, PNNL, Richland, Washington 
Nanoparticles-Based Biosensors for Detection of Environmental Biomarkers

G Shao, Louisiana State University, Baton Rouge, Louisiana

Y Miao, Portland State University, Portland, Oregon

CM Wai, K Shimizu, University of Idaho, Moscow, Idaho

Y Lin, C Timchalk, G Liu, H Wu, J Wang, RC Barry, J Liu, SL Riechers, X Zhang, Z Tang, D Choi, H Wang, A Ma-ham, Pacific Northwest National Laboratory, Richland, Washington

H Liao, Xiamen University, Xiamen, Fujian, China

H Nian, National Tsing Hua University, Hsingchu, Taiwan, Province Of China

K Pinwattana, Chulalongkorn University, Patuwan, Bangkok, Thailand

A Lee, National University of Singapore, Singapore, Malaysia

MH Engelhard, Environmental Molecular Sciences Laboratory, Richland, Washington

D Du, Central China Normal University, Wuhan, Hubei, China

X Kang, Guangdong Ocean University, Guangdong, China

S Zhang, Harbin Institute of Technology, Harbin, China

Y Zhang, Hunan Normal University, Changsha, Hunan, China

\section{Nanoscale Characterization of Nanomaterial - Cell Interactions}

G Orr, BJ Tarasevich, J Teeguarden, J Pounds, JL Phillips, DJ Panther, ME Rosbach, KJ Cassens, Pacific Northwest National Laboratory, Richland, Washington

GA Deitz, PNNL, Richland, Washington

\section{Nanoscale Enzyme Reactors (NERs) for Biofuel Cells}

P Wang, University of Minnesota, Minneapolis, Minnesota

SY Ha, MB Fischback, S Lin, Washington State University, Pullman, Washington

J Kim, Pacific Northwest National Laboratory, Richland, Washington

S Shin, K Kwon, H Park, Korea Advanced Institute of Science and Technology (KAIST), Daejeon, Korea South, Republic Of

NBACC: Host/pathogen interactions (PNNL Scope \# 49488, Lipton’s NBACC Host project)

MS Lipton, Pacific Northwest National Laboratory, Richland, Washington

KK Hixson, Environmental Molecular Sciences Laboratory, Richland, Washington

Near Real-time Alkene Sensor for Atmospheric Aerosol Chemistry Monitoring

JW Grate, Pacific Northwest National Laboratory, Richland, Washington

R Shekarriz, MicroEnergy Technologies, Inc., Portland, Oregon

Neuropeptides and Vasoactive Protein Detection From Human Skin

CT Minson, University of Oregon, Eugene, Oregon

New Theoretical Developments and Computational Studies of Complex Processes in Environmental Chemistry, Waste Containment, and Biochemistry

K Burke, Rutgers University, Piscataway, New Jersey

AE Clark, Washington State University, Pulman, Washington

MC Wander, University of California, Davis, Davis, California

R Devanathan, F Gao, Pacific Northwest National Laboratory, Richland, Washington

S Chumakov, Los Alamos National Laboratory, Los Alamos, New Mexico 
N Marzari, HJ Kulik, N Bonini, B Kozinsky, NE Miller, Massachusetts Institute of Technology, Cambridge, Massachusetts

PA Moreira, Universidade Estadual De Campinas, Sao Paulo, Brazil

MV Losev, RA Evarestov, AI Panin, AV Bandura, St. Petersburg State University, St. Petersburg,

Russian Federation

LR Corrales, JA Knibloe, University of Arizona, Tucson, Arizona

EJ Bylaska, Environmental Molecular Sciences Laboratory, Richland, Washington

A Chartier, Commissariat a L'Energie Nucleair, Gif-sur-Yvette, France

K Tsemekhman, DN Baker, MA Gabriel, WR Schief, University of Washington, Seattle, Washington

J Du, University of North Texas, Denton, Texas

H Jonsson, University of Iceland, Reykjavík, Iceland

AV Morozov, The Rockefeller University, New York, New York

A Cormack, Alfred University, Alfred, New York

T Thonhauser, Wake Forest University, Winston-Salem, North Carolina

Next Generation Clinical Proteomics to Target Human Health Challenges

RD Smith, JM Jacobs, KE Burnum, Pacific Northwest National Laboratory, Richland, Washington

MG Katze, University of Washington, Seattle, Washington

NMR Analysis of Bioprocess Samples

K McAteer, Washington State University, Richland, Washington

SE Baker, Pacific Northwest National Laboratory, Richland, Washington

NMR and Computational Studies of Chemical Transformations at Complex Interfaces

E Iglesia, A Bhan, B Temel, RP Gounder, RT Carr, DA Simonetti, Y Chin, BT Loveless, BM Weiss, University of California, Berkeley, Berkeley, California

J Hu, CH Peden, J Kwak, Y Wang, Pacific Northwest National Laboratory, Richland, Washington

DA Dixon, R Craciun, S Li, M Hernandez Matus, University of Alabama, Tuscaloosa, Tuscaloosa,

Alabama

H Zheng, Dalian Institute of Chemical Physics, Chinese Academy of Sciences, Dalian, China

NMR and EPR Structural Characterization of c-Type Cytochromes and their Complexes from Contaminant Metal Remediating Iron-Respiring Bacteria

TS Magnuson, Idaho State University, Pocatello, Idaho

JR Cort, Pacific Northwest National Laboratory, Richland, Washington

NMR for Catalyst Studies

CH Peden, J Hu, Y Wang, J Kwak, J Szanyi, V Murugesan, Pacific Northwest National Laboratory, Richland, Washington

NMR of Bioprocess Samples

K McAteer, Washington State University, Richland, Washington

DE Culley, SE Baker, Pacific Northwest National Laboratory, Richland, Washington

NMR Study of Ion Transport in Nanostructured Materials

J Liu, K Zhu, Pacific Northwest National Laboratory, Richland, Washington 
NMR Study of Novel Hydrogen Storage Materials

LL Shaw, University of Connecticut, Storrs, Connecticut

Z Yang, J Hu, V Murugesan, J Kwak, Pacific Northwest National Laboratory, Richland, Washington

Non-Destructive Evaluation of Damaged Ancient Scrolls Using NMR and PIXE Scanning to Separate Text From the Scroll Background Combined With 3D Virtual Unrolling

WB Seales, RF Baumann, MD Field, University of Kentucky, Lexington, Kentucky

E Iuliano, Edward M. Iuliano, D.O., Richland, Washington

Non-Empirical Simulations of Nuclear Fuel Materials

P Van Uffelen, E Heifets, J Kotomins, D Grjaznovs, European Commission Joint Research Centre, Eggenstein-Leopoldshafen, Germany

Non-Thermal Reactions in Thin Aqueous Films

GA Kimmel, NG Petrik, Pacific Northwest National Laboratory, Richland, Washington

BO Olanrewaju, Georgia Institute of Technology, Georgia Tech Research Corporation, Atlanta, Georgia

MC Akin, University of Texas at Austin, Austin, Texas

Non-Thermal Reactions in Water Adsorbed on Anion-Doped TiO2(110)

GA Kimmel, NG Petrik, Pacific Northwest National Laboratory, Richland, Washington

Northwest Consortium for Multiscale Mathematics and Application

G Lin, AM Tartakovsky, KF Ferris, Pacific Northwest National Laboratory, Richland, Washington

Novel Catalytic Materials for the Hydrodesulfurization and Water-Gas Shift Processes

ME Bussell, AW Burns, AF Gaudette, MC Smith, Western Washington University, Bellingham,

Washington

NOx Adsorber Materials: Fundamental Studies, and Investigations of Sulfur Poisoning and Thermal Deactivation

CH Peden, RS Disselkamp, D Kim, J Kwak, J Szanyi, RG Tonkyn, CM Verrier, D Tran, JL Male, C Cao, Pacific Northwest National Laboratory, Richland, Washington

MH Engelhard, Environmental Molecular Sciences Laboratory, Richland, Washington

\section{Nucleation, Growth and Evaporation Rates of Aerosols}

SM Kathmann, CJ Mundy, Pacific Northwest National Laboratory, Richland, Washington

TL Windus, MS Gordon, EA Hull, HM Netzloff, ND Atoms, JD Felder, Iowa State University, Ames, Iowa

LD Crosby, National Institute for Computational Sciences, Oak Ridge, Tennessee

Observation of Biogenic Nano-scaled Wire-like Arsenic Sulfide Mineral

JK Fredrickson, Pacific Northwest National Laboratory, Richland, Washington

J Lee, H Hur, Gwangju Institute of Science and Technology (GIST), Gwangju, Korea South, Republic Of

Obtaining Accurate Energetics for Gas-Phase Bimolecular Nucleophilic Substitution (SN2)

Reactions

WL Hase, Texas Technical University, Lubbock, Texas

WA De Jong, Environmental Molecular Sciences Laboratory, Richland, Washington 
Open Ocean Ship-Board Study of Atmospheric Aerosols at the Equatorial Pacific: Characterizing Iron in Marine Aerosol

Y Dessiaterik, Colorado State University, Fort Collins, Colorado

V Shutthanandan, C Wang, Z Zhu, A Laskin, Environmental Molecular Sciences Laboratory, Richland, Washington

AM Johansen, LS Shank, Central Washington University, Ellensburg, Washington

JW Murray, University of Washington, Seattle, Washington

Overall Cleanup of Warm Syngas

L Li, B Chen, CJ Howard, Z Nie, Pacific Northwest National Laboratory, Richland, Washington

Overall Warm Syngas Cleanup

L Li, J Liu, CJ Howard, B Chen, Z Nie, W Liu, Y Su, Pacific Northwest National Laboratory, Richland, Washington

Oxide Clusters - Electronic Structure and Chemical Bonding Using Photoelectron Spectroscopy

H Zhai, W Huang, L Wang, Washington State University, Pullman, Washington

C Ning, Tsinghua University, Beijing, China

PD Dau, Brown University, Providence, Rhode Island

S Li, Xinzhou Teachers University, Xinzhou City, Shanxi, China

L Wang, Washington State University Tri-Cities, Richland, Washington

Oxide Surface Structure and Acidity

KM Rosso, SN Kerisit, Pacific Northwest National Laboratory, Richland, Washington

BR Bickmore, Brigham Young University, Provo, Utah

DM Smith, Whitman College, Walla Walla, Washington

Oxygen Isotope Fractionation in Phosphate During Iron Oxide-Phosphate Interaction

DP Jaisi, RE Blake, Yale University, New Haven, Connecticut

\section{Parallelizing Support Vector Machines}

PJ Nichols, CS Oehmen, Pacific Northwest National Laboratory, Richland, Washington

KA Glass, Environmental Molecular Sciences Laboratory, Richland, Washington

Pathways and Fate of Individual Nanoparticles in Vivo; A Whole Organism Approach

R Tanguay, Oregon State University, Corvallis, Oregon

KJ Cassens, G Orr, Pacific Northwest National Laboratory, Richland, Washington

Peptide Biomarker Analysis

DS Wunschel, Pacific Northwest National Laboratory, Richland, Washington

Phosphoproteome Determination of LPA Treated Osteocytes

NJ Karin, Pacific Northwest National Laboratory, Richland, Washington

Phosphoproteomic Profiling of Breast Cancer Cell Lines

A Umar, Erasmus University Medical Center, Rotterdam, Rotterdam, Netherlands 
Photochemical Processing of Organic Aerosol Particles Studied with High-Resolution Mass Spectrometry

S Nizkorodov, AP Bateman, ML Walser, TB Nguyen, DL Bones, University of California, Irvine, Irivne, California

Y Dessiaterik, Colorado State University, Fort Collins, Colorado

A Laskin, Environmental Molecular Sciences Laboratory, Richland, Washington

Photochemical studies on N-doped TiO2 single crystals - Fundamental Investigations of Water Splitting on Model TiO2 (Mike Henderson's BES Project - 48526)

VH Lam, University of Central Florida, Orlando, Florida

MA Henderson, SA Chambers, S Cheung, T Ohsawa, Z Dohnalek, Pacific Northwest National

Laboratory, Richland, Washington

I Lyubinetsky, T Thevuthasan, P Nachimuthu, Y Du, Environmental Molecular Sciences Laboratory,

Richland, Washington

MK Bowman, University of Alabama, Tuscaloosa, Tuscaloosa, Alabama

R Shao, SC Andrews, unknown, Washington

Y Wang, Northwestern Polytechnical University, Xi'an, Shaanxi, China

Photoemission Electron Microscopy of Small Polaron Formation on $\mathrm{NaCl}$ Islands on Cu (111)

X Zhu, University of Minnesota, Minneapolis, Minnesota

AG Joly, WP Hess, Pacific Northwest National Laboratory, Richland, Washington

KM Beck, Environmental Molecular Sciences Laboratory, Richland, Washington

Photoluminescence and XRD Study of Nanocrystalline Cadmium Sulphide Deposited on the Patterned Surface of Diatom Biosilica

T Gutu, J Jiao, Portland State University, Portland, Oregon

Photometric Response of Anion Selective Chromoionophores

LE Sweet, Pacific Northwest National Laboratory, Richland, Washington

SM Peper, PNNL, Richland, Washington

Photoswitchable Dyes and Nanoparticles Enable High Resolution and Accurate Fluorescent Probes for Cell Imaging

AD Li, Washington State University, Pullman, Washington

D Hu, Pacific Northwest National Laboratory, Richland, Washington

Physicochemical Determinants of Distribution and Fate of Nanoparticles Following in Vivo

Exposure

D Dutta, University of Florida, Gainesville, Florida

J Pounds, T Weber, KJ Cassens, J Teeguarden, G Orr, Pacific Northwest National Laboratory, Richland,

Washington

Pilot Study on Examination of LIRKO Islets and Serum Using Quantitative Proteomic Approaches W Qian, V Petyuk, Pacific Northwest National Laboratory, Richland, Washington

RN Kulkarni, Joslin Diabetes Center, Boston, Massachusetts 


\section{Pipeline to High Performance}

AL Corrigan, Pacific Northwest National Laboratory, Richland, Washington

DE Cowley, D Wang, J Carr, Environmental Molecular Sciences Laboratory, Richland, Washington

\section{Planning Liquid TOFSIMS Experiment}

JP Cowin, L Yang, Pacific Northwest National Laboratory, Richland, Washington

Platelet Proteome for Normal and Type-2 Diabetics

DL Springer, Pacific Northwest National Laboratory, Richland, Washington

\section{Platelet Secretome}

RC Zangar, Pacific Northwest National Laboratory, Richland, Washington

\section{PLFS Testing on Chinook}

EJ Felix, DM Brown, Environmental Molecular Sciences Laboratory, Richland, Washington

Posttranslational Modifications for Early Detection of Breast Cancer

RC Zangar, Pacific Northwest National Laboratory, Richland, Washington

Preliminary Proteomics of Microbial-Host Systems

AT Wright, JN Adkins, AA Schepmoes, MC Burnet, Pacific Northwest National Laboratory, Richland, Washington

Preliminary Test of OPO Experiments Using QPM Frequency Convertors for the Development of Tunable IR Light Source

F Ohuchi, University of Washington, Seattle, Washington

N YU, Gwangju Institute of Science and Technology (GIST), Buk-ku, Gwangju, Korea South, Republic Of

J Ro, Pusan National University Hospital, Busan, Korea South, Republic Of

\section{Preparation of Novel Catalysts using Ion Soft-Landing}

RG Cooks, Purdue University, West Lafayette, Indiana

J Laskin, Q Hu, Pacific Northwest National Laboratory, Richland, Washington

Z Yang, University of Colorado Boulder, Boulder, Colorado

W Peng, National Dong Hwa University, Shoufeng, Hualien, Taiwan, Province Of China

MS Lysonski, Carleton College, Northfield, Minnesota

GE Johnson, PJ Roach, PNNL, Richland, Washington

Preparation of Stoichiometric TiO2(110)-(1x1) Surface

A Bondarchuk, Fritz-Haber Institut der Max Planck Gesellschaft, Berlin, Germany

Preparation of Stoichiometric TiO2(110)-(1x1) Surface

A Bondarchuk, H Freund, Fritz-Haber Institut der Max Planck Gesellschaft, Berlin, Germany 
Probing a Single Crystal Wide Gap Dilute Magnetic Semiconductor: Mn doped $\hat{\mathbf{I}}^{2}$-Ga2O3

TC Lovejoy, MA Olmstead, F Ohuchi, University of Washington, Seattle, Washington

Probing atmospheric chemistry of aerosols to gain insights into cloud condensation behavior A Laskin, C Wang, PL Gassman, Environmental Molecular Sciences Laboratory, Richland, Washington KA Prather, L Russell, RC Sullivan, M Moore, University of California, San Diego, San Diego, California

MK Gilles, RC Moffet, Lawrence Berkeley National Laboratory, Berkeley, California

Probing the Dynamics of a Protein Hydrophobic Core at Low Temperatures

L Vugmeyster, University of Alaska at Anchorage, Anchorage, Alaska

Probing the Electronic Structures of Atomic Clusters and Solution Phase Species in the Gas Phase X Wang, J Yang, NS Parmar, X Xing, W Huang, L Wang, Washington State University, Pullman, Washington

H Woo, The Scripps Research Institute, La Jolla, California

C Ning, Tsinghua University, Beijing, China

PD Dau, Brown University, Providence, Rhode Island

ER Vorpagel, Environmental Molecular Sciences Laboratory, Richland, Washington

C Chi, Fudan University, Shanghai, China

S Li, Xinzhou Teachers University, Xinzhou City, Shanxi, China

L Wang, Washington State University Tri-Cities, Richland, Washington

G Xiao, Xi' an Jiaotong University, Xi'an, Shaanxi, China

Probing the Reactivity of Organic Particles Using Ion Soft-Landing

S Nizkorodov, DL Bones, University of California, Irvine, Irivne, California

J Laskin, Q Hu, Pacific Northwest National Laboratory, Richland, Washington

A Laskin, Environmental Molecular Sciences Laboratory, Richland, Washington

MS Lysonski, Carleton College, Northfield, Minnesota

GE Johnson, PNNL, Richland, Washington

Product Analysis of Reactions of a Model System for Organics on Atmospheric Particles:

Ozonolysis of Self-Assembled Monolayers on Silicon Surfaces

TM McIntire, University of California, Irvine, Irvine, California

Prophylactic Cancer Vaccine

T Liu, Pacific Northwest National Laboratory, Richland, Washington

Proposal for Development of Mass Closure via PNNL PESA for Continuous Fine, Very fine, and Ultra-fine Aerosol Sampling for Health Impact Studies

TA Cahill, University of California, Davis, Davis, California

Proposal for the Development of Capability for Scanning-Probe Infrared

Spectroscopic Imaging with Nanometer Spatial Resolution, MB Raschke, University of Washington, Seattle, Washington 


\section{Protein Complex Identification Using Novel Affinity Resins}

MU Mayer-Cumblidge, L Shi, TC Squier, P Yan, Pacific Northwest National Laboratory, Richland, Washington

S Verma, unknown, Washington

Protein Complexes Altered by Oxidative Stress and Determined by FTICR-MS

HS Smallwood, St. Jude Children's Research Hospital, Memphis, Tennessee

Protein Interactions and Interfaces

G Montelione, Rutgers University, New Brunswick, New Jersey

MA Kennedy, TA Ramelot, Miami University, Oxford, Ohio

A Yee, University of Toronto (Univ. Health Network), toronto, Ontario, Canada

JR Cort, Pacific Northwest National Laboratory, Richland, Washington

PJ Alperin, University of Rochester, Rochester, New York

TW Wietsma, Environmental Molecular Sciences Laboratory, Richland, Washington

C Arrowsmith, University of Toronto, Toronto, Ontario, Canada

Protein Structure and Surface Interactions of an Enamel Protein

DL Masica, Johns Hopkins University, Baltimore, Maryland

GW Buchko, WJ Shaw, Pacific Northwest National Laboratory, Richland, Washington

Proteome Investigations to enable DOE Office of Biomass Programs goals.

EA Panisko, Pacific Northwest National Laboratory, Richland, Washington

Proteomic Analysis of Differential Growth in Desulfovibrio spp. for a Systems Biology Level Investigation.

JD Wall, DA Elias, BJ Giles, University of Missouri, Columbia, Columbia, Missouri

Proteomic Analysis of Extracellular Appendages Displayed in Desulfovibrio vulgaris

DA Elias, University of Missouri, Columbia, Columbia, Missouri

YA Gorby, J. Craig Venter Institute, La Jolla, California

Proteomic Characterization of In Vivo and In Vitro Model Systems of Hepatitis C Virus Infection: JM Jacobs, Pacific Northwest National Laboratory, Richland, Washington

MG Katze, DL Diamond, University of Washington, Seattle, Washington

Proteomic Comparison of Lyophilized and Fresh Human Plasma

W Qian, Pacific Northwest National Laboratory, Richland, Washington

Proteomic Studies of Inflammation and the Host Response to Injury

LL Moldawer, University of Florida, Gainesville, Florida

W Qian, BO Petritis, Pacific Northwest National Laboratory, Richland, Washington

Proteomic-Based Analysis of Components Involved in Human Cytomegalovirus US28-induced Cellular Migration

SM Varnum, Pacific Northwest National Laboratory, Richland, Washington

DN Streblow, Oregon Health Sciences University/Oregon Graduate Institute, Beaverton, Oregon 
Proteomic-Based Mechanistic Analysis of Components Involved in the Immunoprotective Pathways Regulated by Low-Dose Radiation and the Medicinal Mushroom Trametes Versicolor CA Sacksteder, Pacific Northwest National Laboratory, Richland, Washington CA Wenner, M Martzen, Bastyr University, Kenmore, Washington

Proteomics Methods and QSAR Models to Predict Nanoparticle Surface Chemistry Interactions T Liu, Pacific Northwest National Laboratory, Richland, Washington

Proton and Carbon Chemical Shift Assignments of Brodifacoum JR Cort, HM Cho, Pacific Northwest National Laboratory, Richland, Washington

PTR-ITMS: Next Generation Aircraft Instrumentation (Alexander's ASP, PNNL Scope \#47789) SP Garland, University of California, Davis, Redding, California R Zaveri, CM Berkowitz, Pacific Northwest National Laboratory, Richland, Washington MK Newburn, Environmental Molecular Sciences Laboratory, Richland, Washington

Pulsed Multiple Laser Deposition Facility for the Growth of Thin Films in Interfacial Combinatorial Material Exploration

KM Beck, Environmental Molecular Sciences Laboratory, Richland, Washington

F Ohuchi, E Venkatasubramanian, University of Washington, Seattle, Washington

QMP Based Tunable IR Wavelength Conversion Devices For Dental Applications

F Ohuchi, TN Aw, University of Washington, Seattle, Washington

K Kitamura, National Institute for Materials Science, Ibaraki, Japan

Quantitative Characterization of Protein Post-Translational Modifications Using Mass Spectrometry

W Qian, S Ding, Q Zhang, T Heibeck, Pacific Northwest National Laboratory, Richland, Washington

Quantitative Prediction of Kink Site Formation Rate on Calcite and Barite Using Metadynamics JR Rustad, University of California, Davis, Davis, California

GK Schenter, SN Kerisit, Pacific Northwest National Laboratory, Richland, Washington

AG Stack, Georgia Institute of Technology, Georgia Tech Research Corporation, Atlanta, Georgia

SC Parker, University of Bath, Bath, United Kingdom

Quantitative Proteomic Analysis of Bone Cells

NJ Karin, Pacific Northwest National Laboratory, Richland, Washington

Quorum Sensing in a Dissimilatory Metal Reducing Bacterium

BH Lower, Ohio State University, Columbus, Ohio

MF Hochella, DR Learman, Virginia Polytechnic Institute, Blacksburg, Virginia

Radical Mechanisms for Antibiotic and Herbicide Biosynthesis

SC Wang, ML DeLine, KD Allen, Washington State University, Pullman, Washington

Raman Capability for EMSL Users

NJ Hess, Pacific Northwest National Laboratory, Richland, Washington 
RBS, XRD, TOF-SIMS, and AFM on Si-Wafer Supported Vanadium Oxide Model Catalysts AG Getsoian, AT Bell, University of California, Berkeley, Berkeley, California

Reactive Spectro-microscopy Studies of Substrate-Deposited of Organic Aerosol Particles A Tivanski, JA Keck, S Ghorai, University of Iowa, Iowa City, Iowa

A Laskin, PL Gassman, Environmental Molecular Sciences Laboratory, Richland, Washington

Real-Time FTIR Measurements of Fe-oxide Transformation in Presence of Dissimilatory FeReducing Bacteria

GG Geesey, Montana State University, Bozeman, Montana

TC Droubay, Pacific Northwest National Laboratory, Richland, Washington

Real-Time Imaging of Lignocellulosic Biomass Degradation by Fluorescence Microscopy and Atomic Force Microscopy

D Hu, Z Dai, KR Minard, L Wang, ZC Zhang, Pacific Northwest National Laboratory, Richland, Washington

Reduction of Technetium by Fe(II) Associated With Reduced Clay Mineral Nontronite H Dong, ME Bishop, Miami University, Oxford, Ohio

R Kukkadapu, Environmental Molecular Sciences Laboratory, Richland, Washington

Reduction of U6+ by Magnetite and the Rate of Electron Transfer

SN Kerisit, FN Skomurski, KM Rosso, Pacific Northwest National Laboratory, Richland, Washington

RC Ewing, U Becker, University of Michigan, Ann Arbor, Michigan

Reductive Transformation of Iron Oxides: Coupled Solution and Solid-State Pathways

GV Gibbs, Virginia Polytechnic Institute, Blacksburg, Virginia

KM Rosso, S Yanina, SN Kerisit, D McCready, TC Droubay, PP Zarzycki, J Yu, Pacific Northwest

National Laboratory, Richland, Washington

P Meakin, Idaho National Engineering and Environmental Laboratory (INEEL), Idaho Falls, Idaho

C Wang, MH Engelhard, B Arey, Environmental Molecular Sciences Laboratory, Richland, Washington

Refroming of Hydrocarbons on Solid Oxide Fuel Cell Anodes

A Karim, JJ Strohm, DL King, Y Wang, Pacific Northwest National Laboratory, Richland, Washington

Y Yang, University of Washington, Seattle, Washington

X Wang, New Jersey Institute of Technology, Newark, New Jersey

Relating Nanostructure to Composite Properties Via 3D TEM Imaging

J Simonsen, Oregon State University, Corvallis, Oregon

Reliable Electronic Structure Prediction of Molecular Properties

KA Peterson, T Ichiye, Washington State University, Washington, Wash DC

$\mathrm{C} \mathrm{Ng}$, University of California, Davis, Davis, California

HF Schaefer, MC Lind, JM Turney, NM Marshall, JB Ingels, LD Speakman, NJ Stibrich, University of Georgia, Athens, Georgia

SS Xantheas, J Li, GS Fanourgakis, CJ Mundy, S Yoo, Pacific Northwest National Laboratory, Richland, Washington 
BP Hay, Oak Ridge National Laboratory, Oak Ridge, Tennessee

I Dabkowska, University of Gdansk, Gdansk, Poland

JC Werhahn, Technische Universität München, Garching, Germany

S Niu, Georgetown University, Washington, Wash DC

DA Dixon, University of Alabama, Tuscaloosa, Tuscaloosa, Alabama

AK Wilson, NJ DeYonker, University of North Texas, Denton, Texas

M Haranczyk, Lawrence Berkeley National Laboratory, Berkeley, California

RA Bachorz, Universitaet Karlsruhe, Karlsruhe, Germany

MS Gutowski, A Whiteside, Heriot-Watt University, Edinburgh, United Kingdom

S Iwata, Toyota Physical and Chemical Research Institute, Aichi, Japan

L Wang, Washington State University Tri-Cities, Richland, Washington

Reliable Relativistic Quantum Chemistry Calculations for Molecules with Heavy Elements

WA Goddard, V Bryantsev, California Institute of Technology, Pasadena, California

J Autschbach, MT Sterzel, BC Mort, MA Rudolph, Research Foundation University at Buffalo, Buffalo,

New York

BE Bursten, JL Sonnenberg, T Yang, Ohio State University, Columbus, Ohio

AE Clark, C Qin, Washington State University, Pullman, Washington

C Zhan, University of Kentucky, Lexington, Kentucky

IA Infante, Universiteit Amsterdam, Amsterdam, Netherlands

Z Zhang, Stanford University, Stanford, California

J Li, MC Perkins, PJ Nichols, V Glezakou, Pacific Northwest National Laboratory, Richland, Washington

ER Batista, Los Alamos National Laboratory, Los Alamos, New Mexico

BP Hay, Oak Ridge National Laboratory, Oak Ridge, Tennessee

KE Gutowski, University of Notre Dame, South Bend, Indiana

ML Mckee, H Sayin, Auburn University, Auburn, Alabama

L Visscher, SJ Van Gisbergen, C Jacob, Vrijie Universiteit/Scientific Computing and Modelling, Amsterdam, Netherlands

KG Dyall, Schrodinger, Inc., Portland, Oregon

WA De Jong, N Govind, Environmental Molecular Sciences Laboratory, Richland, Washington

TL Windus, GE Schoendorff, Iowa State University, Ames, Iowa

DA Dixon, R Craciun, University of Alabama, Tuscaloosa, Tuscaloosa, Alabama

AK Wilson, University of North Texas, Denton, Texas

SM Walker, University of Manitoba, Winnipeg, Quebec, Canada

A Yakovlev, Scientific Computing and Modeling N.V., Amsterdam, The Netherlands, Netherlands

H Joo, University of the pacific, Stockton, California

LG De Macedo, Sao Paulo State University, Bauru, SP, Brazil

MJ Kullman, Wichita State University, Wichita, Kansas

WC Ermler, Self (Ermler), San Antonio, Texas

Response of Radiation Detector Materials to Ions

BD Milbrath, Pacific Northwest National Laboratory, Richland, Washington

W Zhang, Peking University, Beijing, China

Reversible Organic Derivatization of Silica: Development of New Methods for Creating Template Imprinted Surfaces

JP Peters, RM Jorgensen, Central Washington University, Ellensburg, Washington 
Role of Human Cytomegalovirus in the Acceleration of Vascular Disease

JN Brown, DG Camp, JM Jacobs, RD Smith, Pacific Northwest National Laboratory, Richland, Washington

J Nelson, Oregon Health Sciences University/Oregon Graduate Institute, Portland, Oregon

Role of Microenvironments and Transition Zones in Subsurface Reactive Contaminant Transport S Fendorf, Stanford University, Stanford, California

NA Smet, AL Ward, L Shi, A Konopka, TC Droubay, J Mckinley, O Qafoku, SN Kerisit, KM Rosso, Z Wang, C Liu, JK Fredrickson, JM Zachara, Pacific Northwest National Laboratory, Richland,

Washington

DR Baer, MH Engelhard, B Arey, R Kukkadapu, TW Wietsma, AR Felmy, Environmental Molecular

Sciences Laboratory, Richland, Washington

RP Ewing, Iowa State University, Ames, Iowa

CI Pearce, PNNL, Richland, Washington

Role of Stress in Oxide Wetting of Metal Particles in Ceria-Zirconia-Supported Pd Catalysts

OK Ezekoye, X Pan, GW Graham, University of Michigan, Ann Arbor, Michigan

Role of Surface and Interface on Transport Properties in Nanoscale Conducting Oxides

X Zhou, JW Templeton, Pacific Northwest National Laboratory, Richland, Washington

MH Engelhard, Environmental Molecular Sciences Laboratory, Richland, Washington

Roles for Membrane Receptors in Cellular Responses to External Cues; The Case of ErbB4 and Morphology Dynamics

C Lai, The Scripps Research Institute, La Jolla, California

G Orr, KJ Cassens, Pacific Northwest National Laboratory, Richland, Washington

S Vicini

Georgetown University, Washington DC, Wash DC

Rotationally Resolved Infrared Spectroscopy to Determine the Transition Parameters of the nu9 Band of Ethane

MD Venkataraman, College of William and Mary, Hampton, Virginia

Run ScalaBLAST on Proteomics Datasets

CS Oehmen, AD Norbeck, GA Anderson, Pacific Northwest National Laboratory, Richland, Washington ER Vorpagel, Environmental Molecular Sciences Laboratory, Richland, Washington

\section{Scaling Up for Large Metagenomic Computations With ScalaBLAST}

TP Straatsma, J Nieplocha, CS Oehmen, AL Corrigan, AR Shah, Pacific Northwest National Laboratory, Richland, Washington

HJ Sofia, Whitman College, Walla Walla, Washington

DJ Baxter, Environmental Molecular Sciences Laboratory, Richland, Washington

P Zuber, Oregon Health Sciences University/Oregon Graduate Institute, Beaverton, Oregon

E Szeto, VM Markowitz, Lawrence Berkeley National Laboratory, Berkeley, California

P Hugenholtz, NC Kyrpides, Joint Genome Institute, Walnut Creek, California

A Gosney, PNNL, Richland, Washington 
Scanning and Transmission Electron Microscopy of Grape Berry Shrivel BR Bondada, Washington State University, Richland, Washington

Seattle Structural Genomics Center for Infectious Diseases

GW Buchko, Pacific Northwest National Laboratory, Richland, Washington

$\mathrm{G}$ Varani, University of Washington, Seattle, Washington

Seattle Structural Genomics Center for Infectious Diseases - April 1, 2009 - Oct.1 2009.

GW Buchko, Pacific Northwest National Laboratory, Richland, Washington

G Varani, University of Washington, Seattle, Washington

Secondary Ion Mass Spectrometry to Measure Residual Impurities in Aluminum Nitride Single

Crystals

JH Edgar, Kansas State University, Manhattan, Kansas

Secondary Ion Mass Spectroscopy Study of Transitions Metals Doped ZnO Nanowires

N Athavan, R Könenkamp, Portland State University, Portland, Oregon

Secondary Organic Aerosol Formation from Nitrate Photochemistry

BJ Finlayson-Pitts, M Ezell, Y Yu, EA Bruns, SN Johnson, VM Perraud, University of California, Irvine, Irvine, California

A Zelenyuk, R Zaveri, J Yang, T Vaden, Pacific Northwest National Laboratory, Richland, Washington

L Alexander, Environmental Molecular Sciences Laboratory, Richland, Washington

Secondary Organic Aerosol Production in Biomass Combustion Plumes

JL Collett, AS Holden, Colorado State University, Fort Collins, Colorado

A Laskin, Environmental Molecular Sciences Laboratory, Richland, Washington

Secretome Analysis of Environmental Nanoparticle Induced Biomarkers

JM Jacobs, BD Thrall, LM Masiello, SM Varnum, Pacific Northwest National Laboratory, Richland,

Washington

Selective Heterogeneous Catalysis

LS Fifield, JT Bays, Pacific Northwest National Laboratory, Richland, Washington

Self-Assembly and Phase Transitions in Nanofibers and Liposomes

MJ McCullagh, GC Schatz, S Tonzani, ND Winter, O Lee, Northwestern University, Evanston, Illinois

Semi-Experimental Equilibrium Structures for Molecules of Biological Significance

TA Blake, RL Sams, Pacific Northwest National Laboratory, Richland, Washington

NC Craig, oberlin college, Oberlin, Ohio

Set Up a High Resolution LC System for Phosphopeptide Analysis

S Ding, University of Nebraska, Omaha, Nebraska

SGI Altix Allocation for Advanced Computing Technology Lab

J Nieplocha, AS Lipton, CL Rakowski, JA Fort, BJ Palmer, MD Williams, SS Xantheas, PA Medvick, M Ovtchinnikov, Y Fang, M Singhal, ML Stewart, SM Robinson, AM Tartakovsky, DG Chavarria, 
DH Bacon, VL Freedman, GS Fanourgakis, J Li, LL Nuffer, M Krishnan, A Marquez, CS Oehmen, PD Ellis, WA Perkins, TR Shippert, S Kabilan, T Fu, H Silva, DR Einstein, SJ Bohn, DP Chassin, C Scherrer, G Lin, DR Rector, KP Recknagle, EM Ryan, Pacific Northwest National Laboratory, Richland, Washington

EJ Bylaska, D Wang, Environmental Molecular Sciences Laboratory, Richland, Washington

EA Butterworth, University of Washington, Seattle, Washington

Z Yang, University of Colorado Boulder, Boulder, Colorado

Simultaneous Optical \& Chemical Measurements of Aerosols as a Function of Relative Humidity: Bridging Laboratory and Field Measurements to Models

A Zelenyuk, T Vaden, Pacific Northwest National Laboratory, Richland, Washington

MK Dubey, Los Alamos National Laboratory, Los Alamos, New Mexico

\section{Single Enzyme Nanoparticles}

P Wang, University of Minnesota, Minneapolis, Minnesota

J Kim, JW Grate, Pacific Northwest National Laboratory, Richland, Washington

$\mathrm{H}$ Na, Seoul National University, Seoul, Korea South, Republic Of

S Shin, H Park, H Chang, Korea Advanced Institute of Science and Technology (KAIST), Daejeon, Korea South, Republic Of

S Kim, Pennsylvania State University, University Park, Pennsylvania

\section{Single Nanoparticle Photoluminescence Imaging of TiO2 Trap States}

FJ Knorr, JL McHale, Washington State University, Pullman, Washington

\section{Site-Specific Sample Preparation of Surfaces and Buried Interfaces for TEM and Atom Probe} Analysis

LE Thomas, MJ Olszta, Pacific Northwest National Laboratory, Richland, Washington

B Arey, Environmental Molecular Sciences Laboratory, Richland, Washington

Site-Specific Surface Excitation of Calcium Oxide “Nanocubes”

O Diwald, University of Erlangen-Nuremberg, Erlangen, Germany

\section{Soft-Landing of Complex Ions on Surfaces}

JR Green, RG Cooks, MP Goodwin, Purdue University, West Lafayette, Indiana

J Laskin, O Hadjar, P Wang, JH Futrell, AS Lea, Q Hu, Pacific Northwest National Laboratory, Richland, Washington

Z Zhu, PL Gassman, Environmental Molecular Sciences Laboratory, Richland, Washington

Z Yang, University of Colorado Boulder, Boulder, Colorado

W Peng, National Dong Hwa University, Shoufeng, Hualien, Taiwan, Province Of China

MS Lysonski, Carleton College, Northfield, Minnesota

Solid State 11B-NMR Analysis of the Decomposition Products of Ammonia-borane Thermolysis in Ionic Liquids for Hydrogen Storage: Part 2

DW Himmelberger, ER Berkeley, LG Sneddon, University of Pennsylvania, Philadelphia, Pennsylvania 
Solid-State 29Si\{27Al\} TRAPDOR-MAS and CP-MAS 29Si NMR Experiments on the Atomic Structural Environments of Aluminum and Silicon in Siliceous Sinters

NW Hinman, University of Montana, Missoula, Montana

Solid-State NMR Characterization of Semiconductor Nanoparticle/Polymer Interfaces

MP Espe, SY Ortiz, University of Akron, Akron, Ohio

Solid-State NMR Spectroscopy on Radioactive Samples with Microcoils

I Farnan, University of Cambridge, Cambridge, United Kingdom

D Sakellariou, CEA Saclay, Gif-sur-Yvette, France

Solid-State NMR Structural Studies of HIV-1 CA Protein Assembly

TE Polenova, Y Han, S Paramasivam, S Sun, University of Delaware, Newark, Delaware

Solution Structure of a 42 kDa "Metal Sensor" CzrA-DNA Complex

AK Alphonse Ignatius, DP Giedroc, Indiana University - Bloomington, Bloomington, Indiana

Soybean Proteomics

G Stacey, TH Nguyen, University of Missouri, Columbia, Columbia, Missouri

Spatially Resolved Proteomic studies of Brains Obtained from Control Mice and Treated Mice Simulating Parkinson's Disease

W Qian, Pacific Northwest National Laboratory, Richland, Washington

Speciation and Photochemistry of Nitrogen Oxides Adsorbed to Environmentally Relevant Surfaces: A Rapid Access Proposal

JD Raff, BJ Finlayson-Pitts, University of California, Irvine, Irvine, California

Specificity of Shewanella Oneidensis Outer Membrane Cytochromes OmcA and OmcB Towards Iron- and Manganese-Oxide Surfaces

BH Lower, Ohio State University, Columbus, Ohio

TC Droubay, L Shi, Pacific Northwest National Laboratory, Richland, Washington

Spectral Library (Sharpe's NNSA, PNNL Scope \#28727)

SW Sharpe, TA Blake, TJ Johnson, RL Sams, DC Scott, RG Tonkyn, Pacific Northwest National

Laboratory, Richland, Washington

Spectroelectrochemical and Computational Studies on Interfacial Electron Transfer in Dyesensitized Solar Cells

H Sun, University of South Dakota, Vermillion, South Dakota

Spectroscopic Confirmation of Enzymatic Oxidation of Structural Fe(II) in Biotite by a Lithoautotrophic Fe(II)-oxidizing Bacterial Culture

EE Roden, University of Wisconsin, Madison, Wisconsin

ES Shelobolina, University of Wisconsin-Madison, Madison, Wisconsin

Spin Electronic Phenomena in Magnetically Doped Perovskites and Complex Oxide Interfaces HY Hwang, University of Tokyo, Kashiwa-shi, Chiba, Japan 
TC Kaspar, SA Chambers, TC Droubay, Pacific Northwest National Laboratory, Richland, Washington C Wang, V Shutthanandan, T Thevuthasan, MH Engelhard, Environmental Molecular Sciences Laboratory, Richland, Washington

L Qiao, PNNL, Richland, Washington

Spin Torque Effects in Multilayer Spin Valve with Perpendicular Magnetic Anisotropy L Saraf, B Arey, Environmental Molecular Sciences Laboratory, Richland, Washington

Y Hou, K Krishnan, A Pakhomov, University of Washington, Seattle, Washington

Spin Valve With Perpendicular Anisotropy for Spin Torque Experiments

A Pakhomov, K Krishnan, University of Washington, Seattle, Washington

Stable Isotope Forensics: Acid Scavenger/ Agent Stabilizer Signatures Development

HW Kreuzer, DC Duckworth, J Wahl, JJ Moran, AJ Carman, Pacific Northwest National Laboratory, Richland, Washington

MK Newburn, Environmental Molecular Sciences Laboratory, Richland, Washington

Stable Isotope Ratios of Cyanides and Phosgene

HW Kreuzer, AJ Carman, JJ Moran, Pacific Northwest National Laboratory, Richland, Washington

L Alexander, Environmental Molecular Sciences Laboratory, Richland, Washington

Stochastic Analysis of Advection-Diffusion-Reaction Systems with Applications to Reactive Transport in Porous Media

G Lin, AM Tartakovsky, KD Jarman, Pacific Northwest National Laboratory, Richland, Washington

Stopping Power Measurements of Heavy Ions Over a Wide Range of Energy

R Linares, Universidade de Sao Paulo, Sao Paulo, Brazil

Y Zhang, Environmental Molecular Sciences Laboratory, Richland, Washington

Strontium in Inorganic Materials Studied with High-Field Solid-State NMR Spectroscopy

KT Mueller, CR Strepka, MC Davis, CA Ryan, Pennsylvania State University, University Park,

Pennsylvania

Structural basis for antibody protection against SEB toxicity

CL Baird, CB Boschek, Pacific Northwest National Laboratory, Richland, Washington

Structural Basis of the Lysis Clock in Bacteriophages: The Holin-Antiholin Interaction of Phage Lambda

CB Hilty, Texas A\&M University, College Station, Texas

Structural Engineering Of the de novo Designed Protein Top7 as a Scaffold for Antigen-Binding TP Straatsma, TA Soares Da Silva, CL Baird, DO Apiyo, CB Boschek, Pacific Northwest National Laboratory, Richland, Washington

Structural Investigation of Human Small Heat Shock Proteins Alpha-Beta-Crystallin and HSP27. R Klevit, P Rajagopal, University of Washington, Seattle, Washington 
Structural Properties of Rare-Earth Doped Wide Bandgap Semiconductor Nanowires

Y Gu, AK Mishra, Washington State University, Pullman, Washington

Structural Studies of a Family of Proteins from the Diurnal Cyanobacteria Cyanothece 51142 that Contain an Unusual Repeated Five-Residues Domain (RFR).

S Ni, MA Kennedy, Miami University, Oxford, Ohio

GW Buchko, Pacific Northwest National Laboratory, Richland, Washington

HB Pakrasi, Washington University in St. Louis, St. Louis, Missouri

Structural Studies of Regulators of Histone Protein Synthesis

R Thapar, University of North Carolina, Buffalo, New York

Structure and Bonding in Actinide Oxides

SD Conradson, Los Alamos National Laboratory, Los Alamos, New Mexico

Structure and Catalytic Activity of Supported Early Transition Metal Oxide Clusters

S Li, Tulane University, New Orleans, Louisiana

Z Dohnalek, BD Kay, Z Zhang, R Rousseau, Z Li, Pacific Northwest National Laboratory, Richland,

Washington

JM White, Y Kim, J Gong, University of Texas at Austin, Austin, Texas

Structure and Dynamics of Ca in Clays, Mineral Phases of Concrete, and Ca-NOM Complexes: Combined Natural Abundance 43Ca NMR and Computational Molecular Modeling Studies

RJ Kirkpatrick, AG Kalinichev, Michigan State University, East Lansing, Michigan

GM Bowers, Alfred University, Alfred, New York

Structure and Recognition in Microbial Membranes Proteins, and DNA

BH Lower, Ohio State University, Columbus, Ohio

JH Miller, A Aceves-Gaona, MB Ernst, A Suleiman, Washington State University, Richland, Washington MA Kennedy, Miami University, Oxford, Ohio

TP Straatsma, M Dupuis, LN Douali, RD Lins, TA Soares Da Silva, J Harper, Pacific Northwest National Laboratory, Richland, Washington

MS Sansom, JP Armitage, JP Pikunic, KL Cox, S Khalid, L Vaccaro, University of Oxford, Oxford, United Kingdom

I Dabkowska, University of Gdansk, Gdansk, Poland

M Valiev, ER Vorpagel, Environmental Molecular Sciences Laboratory, Richland, Washington

P Hobza, Academy of Sciences of the Czech Republic, Prague 6, Czech Republic

M Haranczyk, Lawrence Berkeley National Laboratory, Berkeley, California

RA Bachorz, Universitaet Karlsruhe, Karlsruhe, Germany

MS Gutowski, A Whiteside, S Ling, Heriot-Watt University, Edinburgh, United Kingdom

R Gabdoulline, EML Research gGmbH, Heidelberg, Germany

MW Zacharias, F Sieker, Jacobs University Bremen, Bremen, Germany

Structure-Function Correlations of Proteins in Enamel Formation

WJ Shaw, BJ Tarasevich, GW Buchko, AS Lea, Pacific Northwest National Laboratory, Richland, Washington

ML Snead, University of Southern California, Los Angeles, California 
Structure-Property Relationships in Thin Film Energy Conversion Materials and Coatings DW Matson, PE Burrows, LC Olsen, PM Martin, ME Gross, WD Bennett, CC Bonham, G Graff, ES Mast, SN Kundu, AB Padmaperuma, Ls Sapochak, AL Von Ruden,

PK Koech, H Qiao, JV Ryan, X Cai, J Rainbolt, DJ Gaspar, Y Shin, J Swensen, AG Joly, SA Towne, L Wang, N Chopra, Pacific Northwest National Laboratory, Richland, Washington

MH Engelhard, B Arey, Environmental Molecular Sciences Laboratory, Richland, Washington

Studies of Nanomaterials for Removal of Perchlorate in Water

Y Lin, Z Tang, H Wang, J Wang, H Wu, Pacific Northwest National Laboratory, Richland, Washington $\mathrm{X}$ Kang, Guangdong Ocean University, Guangdong, China

Studies on 1,2,3-trichloropropane (TCP) and its Derivatives

M Dupuis, Pacific Northwest National Laboratory, Richland, Washington

EJ Bylaska, AR Felmy, M Valiev, Environmental Molecular Sciences Laboratory, Richland, Washington DA Dixon, University of Alabama, Tuscaloosa, Tuscaloosa, Alabama

PG Tratnyek, Oregon Health Sciences University/Oregon Graduate Institute, Beaverton, Oregon

Study of Desulfovibrio Vulgaris DsrC, an Essential Protein in Sulfate Respiration

IC Pereira, Instituto de Tecnologia Química e Biológica, Oeiras, Portugal

Study of Temperature-Sensitive Mutants of Yeast Guanylate Kinase

P Ye, Washington State University, Pullman, Washington

JR Cort, Pacific Northwest National Laboratory, Richland, Washington

Study of the Electronic Properties of Graphene-Based Materials

CD Zeinalipour-Yazdi, University of Cyprus, Nicosia, Cyprus

Study of the Electronic Structure of Ditechnetium Heptaoxide (Tc207) by Solid-State NMR Spectroscopy and Ab Initio Computational Methods

HM Cho, Pacific Northwest National Laboratory, Richland, Washington

AP Sattelberger, Argonne National Laboratory, Argonne, Illinois

WA De Jong, Environmental Molecular Sciences Laboratory, Richland, Washington

F Poineau, KR Czerwinski, University of Nevada, Las Vegas, Las Vegas, Nevada

Study of the Initial Oxide Scale Formation and Growth on Haynes 214 Alloy at 800 \& 900C

P Singh, University of Connecticut, Storrs, Connecticut

C Vasil, Pacific Northwest National Laboratory, Richland, Washington

Study of Thermal decomposition of Polyester Fibers Treated with Phosphorus Flame Retardants

S Gaan, G Sun, University of California, Davis, Davis, California

Substrate-Specific Binding of Staphylococcus Adhesins to Solid Surfaces

S Lower, BH Lower, Z Oestreicher, Ohio State University, Columbus, Ohio

C Gassman, Columbia Basin College, Pasco, Washington 
Subsurface Reductive Elimination of Trichloroethylene using Zero-Valent Iron Emplaced by Polymer Solutions

MJ Truex, M Oostrom, MA Covert, G Tartakovsky, Pacific Northwest National Laboratory, Richland, Washington

TW Wietsma, Environmental Molecular Sciences Laboratory, Richland, Washington M Sinha, PNNL, Richland, Washington

Subunit Structures of Arabidopsis RNA Polymerase IV Complexes CS Pikaard, Washington University in St. Louis, Saint Louis, Missouri

L Pasa-Tolic, Environmental Molecular Sciences Laboratory, Richland, Washington

Surface and Bulk Characterization of Ambient Ultrafine Particles

EL Bullock, AM Johansen, C Thomas-Bradley, JM Johnston, TK O'connell, JK Wells, Central

Washington University, Ellensburg, Washington

Surface and Interface Studies of Photochemically Initiated Immobilization

S Chada, M Yan, L Liu, DJ Henry, RR Bard, K Wang, Y Miao, SN Uppalapati, X Wang, Portland State University, Portland, Oregon

MH Engelhard, Environmental Molecular Sciences Laboratory, Richland, Washington

Surface Chemistry of Nanocrystalline Zeolites Probed by Magnetic Resonance Techniques WM Ames, SC Larsen, A Petushkov, University of Iowa, Iowa City, Iowa

MK Bowman, University of Alabama, Tuscaloosa, Tuscaloosa, Alabama

Surface Migration of Additives in Polymers

LA Archer, Z Qian, Cornell University, Ithaca, New York

Surface Plasmon Polariton Dependence on Metal Surface Nano-Structure

RE Peale, JW Cleary, CJ Fredricksen, University of Central Florida, Orlando, Florida

Synthesis of Photoactive Metal Oxide Films for Study Using Time-Resolved X-Ray Techniques PB Hillyard, Stanford University, Stanford, California

KJ Gaffney, JR Bargar, Stanford Synchrotron Radiation Laboratory, Menlo Park, California

Systems Biology of Iron, Carbon, and NitrogenMetabolism in the Marine Diatom Phaeodactylum Tricornutum

AE Allen, J. Craig Venter Institute, San Diego, California

Targeted Delivery of Paclitaxel using Elastin Like Polypeptides for Breast Cancer Therapy

D Raucher, University of Mississippi, Jackson, Mississippi

TEM Analysis of Cell/Fe(III) Oxide Aggregates Produced by a Chemolithoautotrophic Fe(II)Oxidizing, Nitrate-Reducing Enrichment Culture

EE Roden, University of Wisconsin, Madison, Wisconsin

TEM and MDS study of NPSS

K Choi, X Sun, Pacific Northwest National Laboratory, Richland, Washington 
TEM Study of Interfacial Layers in Nb-Clad Stainless Steel

I Bae, J Choi, KS Weil, Pacific Northwest National Laboratory, Richland, Washington

Temperature Dependent High-Resolution UV- and IR-Absorption-Cross-Section Spectra of LowVolatility SOA Precursor Hydrocarbons in the Gas-Phase

TJ Johnson, Pacific Northwest National Laboratory, Richland, Washington

RM Volkamer, MJ Molina, University of California, San Diego, La Jolla, California

Terrestrial Carbon Sequestration Studies

JE Amonette, Y Hu, J Hu, Pacific Northwest National Laboratory, Richland, Washington

D Humphrys, PNNL, Richland, Washington

Test Measurements and Approaches for Enhancing Information Available from Surface Analysis of Oxides, Nanoparticles and Organic Materials

WF Stickle, Hewlett-Packard Company, Corvallis, Oregon

DR Baer, MH Engelhard, Environmental Molecular Sciences Laboratory, Richland, Washington

DG Castner, SD Techane, University of Washington, Seattle, Washington

R Wallace, University of Texas at Dallas, Richardson, Texas

The Aerosol Modeling Testbed

JD Fast, WI Gustafson, EG Chapman, Pacific Northwest National Laboratory, Richland, Washington

DJ Baxter, Environmental Molecular Sciences Laboratory, Richland, Washington

The Characterization of Adsorbed Fe(II) and Its Reactivity With Nitrite

CJ Matocha, University of Kentucky, Lexington, Kentucky

R Kukkadapu, Environmental Molecular Sciences Laboratory, Richland, Washington

The Cloud Nucleating Properties of Aerosols at a Continental Site: CHAPS Study

JN Olfert, Brookhaven National Laboratory, Upton, New York

Y Dessiaterik, Colorado State University, Fort Collins, Colorado

A Laskin, Environmental Molecular Sciences Laboratory, Richland, Washington

MK Gilles, Lawrence Berkeley National Laboratory, Berkeley, California

J Wang, shenyang pharmaceutical university, shenyang, China

The Development of a Compact RF Quadrupole Mass Spectrometer (RFQ-MS) for Germanium Isotope Analysis

R Wang, A Wong, Nonlinear Ion Dynamics, LLC, Panorama City, California

The Effect of Surface Active Organic Molecules on the Water Uptake, Shape and Density of Hygroscopic Particles of Atmospheric Importance

GB Ellison, LA Cuadra-Rodriguez, University of Colorado, Boulder, Colorado

A Zelenyuk, J Yang, T Vaden, Pacific Northwest National Laboratory, Richland, Washington

The Electronic Structure and Dynamics of Photocatalytic and Electronic Interfaces

J Zhao, H Petek, University of Pittsburgh, Pittsburgh, Pennsylvania

The Formation and Structure of Tropical Organic/Water Aerosols

GB Ellison, LA Cuadra-Rodriguez, University of Colorado, Boulder, Colorado 
A Zelenyuk, R Zaveri, J Yang, T Vaden, Pacific Northwest National Laboratory, Richland, Washington Y Dessiaterik, Colorado State University, Fort Collins, Colorado

A Laskin, Environmental Molecular Sciences Laboratory, Richland, Washington

The Influence of Different Fe(III) Phases and the Effect of Sulfate Reduction on the Simultaneous Bioreduction of Iron and Uranium

PR Jaffe, Princeton University, Princeton, New Jersey

The Interaction of $\mathbf{H C l}$ on Water Ice Nanoscale Films

RS Smith, BD Kay, Pacific Northwest National Laboratory, Richland, Washington

P Ayotte, Universite de Sherbrooke/Concordia University (University of Sherbrooke), Sherbrooke,

Quebec, Canada

The Iron Binding Environment of the Cyanobacterial Ferric Ion Transporter, FutA1

TJ Smith, Donald Danforth Plant Science Center, Saint Louis, Missouri

The Proteome of Prostate Cancer-Derived Exosomes: Exosomes As Biomarker Treasure Chests G Jenster, D Duijvesz, Erasmus MC, Rotterdam, Netherlands

The Reduction of Uranyl and Chromate to Insoluble species by Green Rust and other ferrous hydroxide surfaces.

MC Wander, JR Rustad, University of California, Davis, Davis, California

The Relationship between Particle Properties and Ice Nucleation: Laboratory and Field Investigation and Parameterization Development

X Liu, A Zelenyuk, SJ Ghan, J Yang, T Vaden, Pacific Northwest National Laboratory, Richland, Washington

The Role of Extracellular Polymeric Substances in Bacterial Fouling of Membranes KM Rosso, Pacific Northwest National Laboratory, Richland, Washington

B Korpol, S Chellam, AR Badireddy, University of Houston, Houston, Texas

The Role of Ice and Aerosol Surfaces in Halogen Activation in the Arctic Springtime Boundary Layer

Y Dessiaterik, Colorado State University, Fort Collins, Colorado

WR Simpson, L Alvarez-aviles, DM Carlson, University of Alaska Fairbanks, Fairbanks, Alaska

A Laskin, V Shutthanandan, Environmental Molecular Sciences Laboratory, Richland, Washington

The Role of PdZn Alloy Formation and Particle Size on the Selectivity for Steam Reforming of Methanol

VM Lebarbier, Pacific Northwest National Laboratory, Richland, Washington

AM Karim, AK Datye, TR Conant, University of New Mexico, Albuquerque, New Mexico

The Role of U(V) During Heterogeneous Reduction of Aqueous U(VI) to U(IV).

ES Ilton, Pacific Northwest National Laboratory, Richland, Washington

MH Engelhard, Environmental Molecular Sciences Laboratory, Richland, Washington

J Boily, Umea University, Umea, Sweden 
The Study of Atomic Gold Clusters in Polyaniline

AH Saheb, M Josowicz, JA Janata, Georgia Institute of Technology, Georgia Tech Research Corporation, Atlanta, Georgia

Theoretical Modeling of Fluorescence Properties in Biological Systems

M Valiev, K Kowalski, Environmental Molecular Sciences Laboratory, Richland, Washington

J Liang, S Matsika, KA Kistler, CR Kozak, Z Lu, Temple University, Philadelphia, Pennsylvania

Thermodynamic and Chemical Properties of La1-xSrxCoxFe1-xO3 from First-Principles

Calculations: Implications for Use as SOFC Cathodes

SB Sinnott, C Lee, University of Florida, Gainesville, Florida

Thin Film Characterization by Rutherford Backscattering (RBS) as a Strong Complement to Our Current Research Efforts

MR Linford, ML Bailey, Brigham Young University, Provo, Utah

Thin Film Electrolyte/Electrode Development and Enhanced Ionic Transport in Miniaturized Solid Oxide Fuel Cells on Silicon Chips

P Singh, University of Connecticut, Storrs, Connecticut

O Marina, Pacific Northwest National Laboratory, Richland, Washington

Three-Dimensional Structural Analysis of Altered Micas by Ion Beam/Scanning Electron Microscopy (FIB/SEM Dual Beam), $\mu$-X-ray Diffraction, and Transmission Electron Microscopy J Mckinley, Pacific Northwest National Laboratory, Richland, Washington B Arey, P Nachimuthu, Environmental Molecular Sciences Laboratory, Richland, Washington

Thyroid Hormone and Myocardial Substrate Use with Aging MA Portman, A Olson, Children's Hospital and Regional Medical Center, Seattle, Washington

Tissue Distribution and Fate of Nanoparticles Following in Vivo Exposure

G Orr, Pacific Northwest National Laboratory, Richland, Washington

K Sarlo, The Procter and Gamble Company, Fairfield, Ohio

Title: Advanced Proteomics and Metabolomics Studies of Type 2 Diabetes and Pre-Diabetes TO Metz, RD Smith, DG Camp, W Qian, MA Gritsenko, Y Kim, GI Mezengie, Pacific Northwest National Laboratory, Richland, Washington

J Baynes, University of South Carolina, Columbia, South Carolina

R Hoffman, University of Leipzig, Leipzig, Germany

JL Coffen, Self (Coffen), Richland, Washington

Todd Allen's Proposal

TR Allen, L Tan, AM Kruizenga, University of Wisconsin-Madison, Madison, Wisconsin

ToF-SIMS Characterization of Ni Films Used to Grow Few-Layer Graphene

J Kong, A Reina Cecco, Massachusetts Institute of Technology, Cambridge, Massachusetts 
Transformation of Meta-Stable Al to Kaolinite: Molecular Structure and Reaction Pathways From Solid-State NMR.

SA Carroll, RS Maxwell, Lawrence Livermore - National Laboratory, Livermore, California JR Houston, California State University, Sacramento, Sacramento, California

Transport Mechanism and Microstructural Effects on Ag and Cs Diffusion in beta-SiC TJ Gerczak, TR Allen, University of Wisconsin-Madison, Madison, Wisconsin

Trauma \& Sepsis Induced Changes in Immune Cell Membrane Receptor Trafficking

J Cuschieri, University of Washington, Seattle, Washington

Trauma-Induced Reprogramming: Changes in Lipid Raft Protein Content

V Petyuk, Pacific Northwest National Laboratory, Richland, Washington

J Cuschieri, University of Washington, Seattle, Washington

TRLIF Studies of Eu(III) Sorption to Quartz and Uranophane

SB Clark, N Wall, AE Clark, Washington State University, Pulman, Washington

Tuning the Chemistry and Configuration of Oxide Nanostructures for Energy and Environmental Applications

S Seal, AS Karakoti, A Kumar, A Vincent, University of Central Florida, Orlando, Florida

DK Belcher, North Pines Middle School, Spokane Valley, Washington

Ultrafast Laser Interactions with Wide Bandgap Materials

JT Dickinson, E Khan, Washington State University, PUllman, Washington

WP Hess, AG Joly, Pacific Northwest National Laboratory, Richland, Washington

Ultra-High Accuracy Simulations for Large Molecular Systems: Exploring the Cutting Edge Limits of NWChem

S Krishnamoorthy, Pacific Northwest National Laboratory, Richland, Washington

JR Hammond, Argonne National Laboratory, Argonne, Illinois

K Kowalski, EJ Bylaska, M Valiev, Environmental Molecular Sciences Laboratory, Richland,

Washington

O Villa, PNNL, Richland, Washington

Ultrastructure of Aerial Interface in Apple

EA Curry, USDA, ARS, Tree Fruit Research Laboratory, Wenatchee, Washington

Uncovering Proteogenomic Patterns on an Evolutionary Gradient of Termite Gut Verrucomicrobia Isolates

JL Rodrigues, The University of Texas at Arlington, Arlington, Texas

Understanding and Development of Novel NanoMaterials for Frequency Upconversion

K Yu, National Research Council of Canada, Ottawa, Ontario, Canada

W Chen, The University of Texas at Arlington, Arlington, Texas 
Understanding Field-Dependent Quadrupolar Splitting of 131Xe for Applications to Surface/Material Studies

KF Stupic, T Meersmann, Colorado State University, Fort Collins, Colorado

Understanding I/O behavior of Scientific Applications

DM Brown, EJ Felix, Environmental Molecular Sciences Laboratory, Richland, Washington

A Kuruvilla, PNNL, Richland, Washington

Understanding Microorganism-Metal Interactions

BE Kimball, SL Brantley, Pennsylvania State University, University Park, Pennsylvania

Understanding of Long-Term Phase Stability and Degradations in Solid Oxide Fuel Cells P Singh, University of Connecticut, Storrs, Connecticut

DL King, LR Pederson, Pacific Northwest National Laboratory, Richland, Washington

N Govindaraju, University of Cincinnati, Cincinnati, Ohio

L Miara, Boston University, Brookline, Massachusetts

Understanding the Dynamics of the Cyclophilins Family of Enzymes and Their Interactions With Their CD147 Receptor.

EZ Eisenmesser, JS Redzic, University of Colorado at Denver, Aurora, Colorado

Understanding the Influence of Radiation Exposure on Ceria Nanoparticle Surface Chemistry S Seal, A Kumar, A Vincent, University of Central Florida, Orlando, Florida

R Devanathan, Pacific Northwest National Laboratory, Richland, Washington

RP Sanghavi, Arizona State University, Tempe, Arizona

Understanding the Mechanisms and Enhancement of Photostimulated Luminescence by Nanoscale Design and Fabrication

W Chen, The University of Texas at Arlington, Arlington, Texas

Understanding the Structure-Catalytic Function Relationship of Cobalt Based Catalysts for Steam Reforming of Oxygenated Hydrocarbons

SY Ha, S Lin, Washington State University, Pullman, Washington

Understanding the Structure-Catalytic Function Relationship of Cobalt Based Catalysts for Steam Reforming of Ethanol

SY Ha, S Lin, Washington State University, Pullman, Washington

Undoped and n-type Doped TiO2 Anatase Surface Structures and Reactivity with Water

S Chamberlin, University of Wisconsin, Milwaukee, Wisconsin

CJ Hirschmugl, University of Wisconsin - Milwaukee, Milwaukee, Wisconsin

Unraveling Cellular Controls on the Diversity of Mn Oxides Formed By Fungi

CM Hansel, CM Santelli, Harvard University, Cambridge, Massachusetts

Use of Biofluids and a Unique Inducible/Reversible Mouse Model of Aggressive Neoplasia to Test for Cancer Biomarkers

FM Morrish, DM Hockenbery, Fred Hutchinson Cancer Research Center, Seattle, Washington 
Using High Resolution TEM to Aquire Uranium Speciation Information of Environmental Relevance

M VanEngelen, B Peyton, Montana State University, Bozeman, Montana

Using Metallic Interlayers to Stabilize Metal-Metal Interfaces

RJ Smith, WA Priyantha, A Comouth, H Chen, Montana State University, Bozeman, Montana

Using the Atmosphere as a Kinetics Laboratory: Investigation of Rapid Exhaust Plume Chemistry using Proton Transfer Reaction Mass Spectrometry

WB Knighton, Montana State University, Bozeman, Montana

SC Herndon, Aerodyne Research Inc, Billerica, Maine

V-51 Solid-State NMR and Density Functional Theory Studies of Vanadium Haloperoxidases

TE Polenova, S Bolte, S Sun, S Paramasivam, University of Delaware, Newark, Delaware

Validation of Whole-Core Determination of Soil Hydraulic Properties

MJ Fayer, Pacific Northwest National Laboratory, Richland, Washington

Variable-Temperature Mössbauer Spectroscopy of Aluminum-Substituted Maghemites

JM Bigham, Ohio State University, Columbus, Ohio

MA Batista, Universidade Estadual de Maringa, Columbus, Brazil

Visualization of a Pd-Au\{100\} Catalyst: a Scanning Tunneling Microscopy Study

P Han, DW Goodman, F Yang, Texas A\&M University, College Station, Texas

Visualization of a Pt-Co(111) Catalyst: a Scanning Tunneling Microscopy Study

F Yang, DW Goodman, Texas A\&M University, College Station, Texas

WaNPRC Macaque Proteomics for Models of Influenza Infection and AIDS

DG Camp, Pacific Northwest National Laboratory, Richland, Washington

Wettability And Mass Transfer Effects on Flow and Transport of Mixed DNAPL in Hanford Site Sediments

C Werth, AJ Valocchi, University of Illinois, Urbana-Champaign, Illinois

M Oostrom, MA Covert, Pacific Northwest National Laboratory, Richland, Washington

TW Wietsma, Environmental Molecular Sciences Laboratory, Richland, Washington

\section{WRI Breast Cancer}

KD Rodland, DG Camp, T Liu, Pacific Northwest National Laboratory, Richland, Washington

RJ Mural, Windber Research Institute, Windber, Pennsylvania

\section{WSU Tricities Electron Microscopy Course}

EE Brown, RM Abdella, Washington State University, Richland, Washington

EC Buck, LA Snow, KE Draper, D Howe, Pacific Northwest National Laboratory, Richland, Washington RL Sontag, Washington State University Tri-Cities, Richland, Washington

MK Edwards, KM Denslow, CC Bovaird, PNNL, Richland, Washington 


\section{XPS Analysis of Oxidized HT9 Alloy Samples}

C Windisch, Pacific Northwest National Laboratory, Richland, Washington

\section{Zinc Oxide: a Material for Efficient Light Emission}

MD Mccluskey, W Hlaing Oo, ST Teklemichael, Washington State University, Pullman, Washington

DA Harper, Amelia L. Johnson High School, Thomaston, Alabama 


\section{Fiscal Year 2009 Publications}

Publications by EMSL staff members and from users where the publication resulted from research carried out at EMSL are listed below.

\section{Journal Articles}

Abid, AD, ED Tolmachoff, DJ Phares, H Wang, Y Liu, and A Laskin. 2009. "Size Distribution and Morphology of Nascent Soot in Premixed Ethylene Flames with and without Benzene Doping." in Proceedings of the Combustion Institute, Vol 32, pp. 681-688. Elsevier, New York.

Aiken, A, D Salcedo, MJ Cubison, J Huffman, P DeCarlo, IM Ulbrich, KS Docherty, DT Sueper, J Kimmel, DR Worsnop, A Trimborn, M Northway, EA Stone, JJ Schauer, RM Volkamer, E Fortner, B de Foy, J Wang, A Laskin, V Shutthanandan, J Zheng, R Zhang, JS Gaffney, NA Marley, GL ParedesMiranda, WP Arnott, LT Molina, G Sosa, and JL Jimenez. 2009. "Mexico City Aerosol Analysis During Milagro Using High Resolution Aerosol Mass Spectrometry at the Urban Supersite (T0). Part 1: Fine Particle Composition and Organic Source Apportionment." Atmospheric Chemistry and Physics 9(17):6633-53.

Akin, MC, NG Petrik, and GA Kimmel. 2009. "Electron-Stimulated Reactions and O-2 Production in Methanol-Covered Amorphous Solid Water Films." Journal of Chemical Physics 130(10):Art. No. 104710 .

Alquier, T, M-L Peyot, MG Latour, M Kebede, CM Sorensen, S Gesta, CR Kahn, RD Smith, TL Jetton, TO Metz, M Prentki, and VJ Poitout. 2009. "Deletion Gpr40 Impairs Glucose-Induced Insulin Secretion in Vivo in Mice without Affecting Intracellular Fuel Metabolism in Islets." Diabetes

Andersen A, N Govind, and L Subramanian. 2008. "Theoretical Study of the Mechanism Behind the para-Selective Nitration of Toluene in Zeolite H-Beta." Molecular Simulation 34(10-15):1025-1039. doi:10.1080/08927020802191958

Aubriet, F, J-J Gaumet, WA De Jong, GS Groenewold, AK Gianotto, ME Mcliwain, MJ Van Stipdonk, and CM Leavitt. 2009, "Cerium Oxyhydroxide Clusters: Formation, Structure and Reactivity." Journal of Physical Chemistry A 113(22):6239-52.

Badireddy AR, BR Korpol, S Chellam, PL Gassman, MH Engelhard, AS Lea, and KM Rosso. 2008. "Spectroscopic Characterization of Extracellular Polymeric Substances from Escherichia coli and Serratia marcescens: Suppression using Sub-Inhibitory Concentrations of Bismuth Thiols." Biomacromolecules 9(11):3079-3089. doi:10.1021/bm800600p

Bae, I-T, W Jiang, CM Wang, WJ Weber, and Y Zhang. 2009, “Thermal Evolution of Microstructure in Ion-Irradiated Gan.” Journal of Applied Physics 105(8):083514, 1-7.

Baer, DR, MH Engelhard, AR Felmy, JJ Ford, JZ Hu, AS Lea, P Nachimuthu, LV Saraf, JA Sears, and S Thevuthasan. 2009, "New Approaches for Characterizing Sensor and Other Modern Complex Materials.”ECS Transactions 19(6):137-48. 10.1149/1.3118546 
Barry, RC, Y Lin, J Wang, G Liu, and C Timchalk. 2009. "Nanotechnology-Based Electrochemical Sensors for Biomonitoring Chemical Exposures." Journal of Exposure Science and Environmental Epidemiology 19(1):1-18.

Bateman AP, ML Walser, Y Dessiaterik, J Laskin, A Laskin, and S Nizkorodov. 2008. "The Effect of Solvent on the Analysis of Secondary Organic Aerosol Using Electrospray Ionization Mass Spectrometry." Environmental Science \& Technology 42(19):7341-7346. doi:10.1021/es801226w

Bateman, AP, S Nizkorodov, J Laskin, and A Laskin. 2009. “Time-Resolved Molecular Characterization of Limonene/Ozone Aerosol Using High-Resolution Electrospray Ionization Mass Spectrometry.” Physical Chemistry Chemical Physics. PCCP (11):7931-42.

Bell, RC, K Wu, MJ Iedema, GK Schenter, and JP Cowin. 2009. “The Oil-Water Interface: Mapping the Solvation Potential." Journal of the American Chemical Society 131(3):1037-1042.

Bickmore, BR, KM Rosso, ID Brown, and SN Kerisit. 2009. "Bond-Valence Constraints on Liquid Water Structure.” Journal of Physical Chemistry A 113(9):1847-1857.

Boschek, CB, DO Apiyo, TA Soares, HE Engelmann, N Pefaur, TP Straatsma, and CL Baird. 2009. "Engineering an Ultra-Stable Affinity Reagent Based on Top7." Protein Engineering, Design \& Selection 22(5):325-332.

Bose, S, MF Hochella, YA Gorby, DW Kennedy, DE McCready, AS Madden, and BH Lower. 2009. "Bioreduction of Hematite Nanoparticles by the Dissimilatory Iron Reducing Bacterium Shewanella Oneidensis Mr-1." Geochimica et Cosmochimica Acta 73(4):962-976.

Bryantsev, VS, MS Diallo, ACT Van Duin, and WA Goddard I. I. I. 2009, "Evaluation of B3lyp, X3lyp, and M06-Class Density Functionals for Predicting the Binding Energies of Neutral, Protonated, and Deprotonated Water Clusters." Journal of Chemical Theory and Computation 5(4):1016-26. $10.1021 / \mathrm{ct} 800549 \mathrm{f}$

Bubin, S, M Stanke, and L Adamowicz. 2009. "Non-Born-Oppenheimer Calculations of the Bh Molecule.” Journal of Chemical Physics 131(4):044128.

Buchko GW, BJ Tarasevich, JG Bekhazi, ML Snead, and WJ Shaw. 2008. “A Solution NMR Investigation into the Early Events of Amelogenin Nanosphere Self-Assembly Initiated with Sodium Chloride or Calcium Chloride.” Biochemistry 47(50):13215-13222. doi:10.1021/bi8018288

Buchko, GW, H Robinson, and A Addlagatta. 2009. "Structural Characterization of the Protein cce_0567 from Cyanothece 51142, a Metalloprotein Associated with Nitrogen Fixation in the DUF683 Family." Biochimica et Biophysica Acta--Proteins and Proteomics 1794(4):627-633.

Burgos WD, J McDonough, JM Senko, G Zhang, A Dohnalkova, SD Kelly, YA Gorby, and KM Kemner. 2008. "Characterization of uraninite nanoparticles produced by Shewanella oneidensis MR-1." Geochimica et Cosmochimica Acta 72(20):4901-4915. doi:10.1016/j.gca.2008.07.016 
Bylaska, EJ, M Holst, and JH Weare. 2009, “Adaptive Finite Element Method for Solving the Exact Kohn-Sham Equation of Density Functional Theory." Journal of Chemical Theory and Computation 5(4):937-48. 10.1021/ct800350j

Chambers, SA, T Ohsawa, CM Wang, I Lyubinetsky, and JE Jaffe. 2009. "Band Offsets at the Epitaxial Anatase TiO2/N-SrTiO3(001) Interface.”Surface Science 603(5):771-780.

Chambers, SA. 2009, "Comment On "Origin of Metallic States at the Heterointerfaces between the Band Insulators Laalo3 and Srtio3”." Physical Review Letters 102(19):Art. No. 199703.

10.1103/PhysRevLett.102.199703

Chang, C-1, S Sankaranarayanan, MH Engelhard, V Shutthanandan, and S Ramanathan. 2009. "On the Relationship between Non-Stoichiometry and Passivity Breakdown in Ultra-Thin Oxides: Combined Depth-Dependent Spectroscopy, Mott-Schottky Analysis and Molecular Dynamics Simulation Studies." Journal of Physical Chemistry C 113(9):3502-3511.

Chen, B, X Ren, T Neville, WG Jerome, DW Hoyt, DL Sparks, G Ren, and J Wang. 2009, "Apolipoprotein AI Tertiary Structures Determine Stability and Phospholipid-Binding Activity of Discoidal High-Density Lipoprotein Particles of Different Sizes.” Protein Science 18(5):921-35. $10.1002 /$ pro. 101

Cholette, F, T Zubkov, RS Smith, Z Dohnalek, BD Kay, and P Ayotte. 2009, "Infrared Spectroscopy and Optical Constants of Porous Amorphous Solid Water.” Journal of Physical Chemistry B 113(13):4131-40.

Chowdhury, SM, L Shi, H Yoon, C Ansong, LM Rommereim, AD Norbeck, KJ Auberry, R Moore, JN Adkins, F Heffron, and RD Smith. 2009. "A Method for Investigating Protein-Protein Interactions Related to Salmonella Typhimurium Pathogenesis." Journal of Proteome Research 8(3):1504-1514.

Cort JR, TA Ramelot, D Murray, T Acton, LC Ma, R Xiao, G Montelione, and MA Kennedy. 2008. "Structure of an Acetyl-CoA Binding Protein from Staphylococcus aureus Representing a Novel Subfamily of GCN5-related N-Acetyltransferase-like Proteins." Journal of Structural and Functional Genomics 9(1-4):7-20. doi:10.1007/s10969-008-9041-z

Cort, JR, and HM Cho. 2009. "1H and 13c NMR Chemical Shift Assignments and Conformational Analysis for the Two Diastereomers of the Vitamin K Epoxide Reductase Inhibitor Brodifacoum." Magnetic Resonance in Chemistry 47(10):897-901.

Coyle, CA, OA Marina, EC Thomsen, DJ Edwards, CN Cramer, GW Coffey, and LR Pederson. 2009, "Interactions of Nickel/Zirconia Solid Oxide Fuel Cell Anodes with Coal Gas Containing Arsenic." Journal of Power Sources 193(2):730-38. 10.1016/j.jpowsour.2009.04.042

Craig NC, and RL Sams. 2008. "An Investigation of the Rotamers of Butadiene by High-Resolution Infrared Spectroscopy.” Journal of Physical Chemistry A 112(49):12637-12646. doi:10.1021/jp807677y

Crosby, LD, SM Kathmann, and TL Windus. 2009, "Implementation of Dynamical Nucleation Theory with Quantum Potentials.” Journal of Computational Chemistry 30(5):743-49. 
Davidson, A, TC Leeper, Z Athanassiou, K Patora-Komisarska, J Karn, JA Robinson, and G Varani. 2009. "Simultaneous Recognition of Hiv-1 Tar Rna Bulge and Loop Sequences by Cyclic Peptide Mimics of Tat Protein." Proceedings of the National Academy of Sciences of the United States of America 106(29):11931-36.

Davis, MC, WJ Brouwer, DJ Wesolowski, LM Anovitz, AS Lipton, and KT Mueller. 2009. "Magnesium Silicate Dissolution Investigated by 29Si MAS, 1H-29Si CP MAS, 25Mg QCPMG, and 1H-25Mg CP QCPMG NMR.” Physical Chemistry Chemical Physics. PCCP 11(32):7013-21.

de Gouw, JA, D Welsh-Bon, C Warneke, WC Kuster, ML Alexander, AK Baker, AJ Beyersdorf, DR Blake, MR Canagaratna, AT Celada, LG Huey, W Junkermann, TB Onasch, A Salcido, SJ Sjostedt, A Sullivan, DJ Tanner, L Vargas-Ortiz, RJ Weber, DR Worsnop, X-Y Yu, and RA Zaveri. 2009, "Emission and Chemistry of Organic Carbon in the Gas and Aerosol Phase at a Sub-Urban Site near Mexico City in March 2006 During the Milagro Study." Atmospheric Chemistry and Physics 9(10):342542.

Deskins, NA, and M Dupuis. 2009. "Intrinsic Hole Migration Rates in TiO2 from Density Functional Theory." Journal of Physical Chemistry C 113(1):346-358.

Deskins, NA, RJ Rousseau, and M Dupuis. 2009. "Localized Electronic States from Surface Hydroxyls and Polarons in TiO2(110)." Journal of Physical Chemistry C 113(33):14583-86.

Deskins, NA. 2009. “Ti 3p Electrons: Core or Valence?” Chemical Physics Letters 471(1-3):75-79.

Devanathan, R, J Yu, and WJ Weber. 2009, "Energetic Recoils in Uo2 Simulated Using Five Different Potentials.” Journal of Chemical Physics 130(17):Art. No. 174502.

Devanathan, R, S Thevuthasan, and JD Gale. 2009, "Defect Interactions and Ionic Transport in Scandia Stabilized Zirconia.” Physical Chemistry Chemical Physics. PCCP 11(26):5506-11.

Ding YHR, KK Hixson, M Aklujkar, MS Lipton, RD Smith, DR Lovley, and T Mester. 2008. "Proteome of Geobacter sulfurreducens grown with Fe(III) oxide or Fe(III) citrate as the electron acceptor." Biochimica et Biophysica Acta--Proteins and Proteomics 1784(12):1935-1941. doi:10.1016/j.bbapap.2008.06.011

Dohnalek Z, J Kim, and BD Kay. 2008. "Understanding How Surface Morphology and Hydrogen Dissolution Influence Ethylene Hydrogenation on Palladium." Journal of Physical Chemistry C 112(40):15796-15801. doi:10.1021/jp803880x

Dreger, ZA, E Balasubramaniam, YM Gupta, and AG Joly. 2009. "High-Pressure Effects on the Electronic Structure of Anthracene Single Crystals: Role of Nonhydrostaticity." Journal of Physical Chemistry A 113(8):1489-1496.

Droubay, T, TC Kaspar, BP Kaspar, and SA Chambers. 2009. “Cation Dopant Distributions in Nanostructures of Transition-Metal Doped ZnO:Monte Carlo Simulations." Physical Review. B, Condensed Matter and Materials Physics 79(7):Art. No. 075324. 
Druhan JL, ME Conrad, KH Williams, AL N'Guessan, PE Long, and SS Hubbard. 2008. "Sulfur Isotopes as Indicators of Amended Bacterial Sulfate Reduction Processes Influencing Field Scale Uranium Bioremediation.” Environmental Science \& Technology 42(21):7842-7849. doi: $10.1021 / \mathrm{es} 800414 \mathrm{~s}$

Du, Y, NA Deskins, Z Zhang, Z Dohnalek, M Dupuis, and I Lyubinetsky. 2009. "Imaging Consecutive Steps of O2 Reaction with Hydroxylated TiO2(110): Identification of HO2 and Terminal OH Intermediates." Journal of Physical Chemistry C 113(2):666-671.

Du, Y, NA Deskins, Z Zhang, Z Dohnalek, M Dupuis, and I Lyubinetsky. 2009. "Two Pathways for Water Interaction with Oxygen Adatoms on TiO2(110)." Physical Review Letters 102(9):Art. No. 096102.

Einstein DR, B Neradilak, N Pollisar, KR Minard, C Wallis, M Fanucchi, JP Carson, AP Kuprat, S Kabilan, R Jacob, and R Corley. 2008. "An Automated Self-similarity Analysis of the Pulmonary Tree of the Sprague-Dawley Rat.” The Anatomical Record 291(12):1628-1648. doi:10.1002/ar.20771

Elliott, DC, and TR Hart. 2009. "Catalytic Hydroprocessing of Chemical Models for Bio-Oil.” Energy and Fuels 23(2):631-637.

Elsasser, BM, M Valiev, and JH Weare. 2009. "A Dianionic Phosphorane Intermediate and Transition States in an Associative AN+DN Mechanism for the Ribonucleasea Hydrolysis Reaction." Journal of the American Chemical Society 131(11):3869-3871.

Epifanovsky E, K Kowalski, PD Fan, M Valiev, S Matsika, and A Krylov. 2008. "On the Electronically Excited States of Uracil.” Journal of Physical Chemistry A 112(40):9983-9992. doi:10.1021/jp803758q

Fast, JD, A Aiken, JD Allan, ML Alexander, T Campos, MR Canagaratna, EG Chapman, P DeCarlo, B de Foy, J Gaffney, JA de Gouw, JC Doran, L Emmons, A Hodzic, SC Herndon, LG Huey, JT Jayne, JL Jimenez, LI Kleinman, WC Kuster, NA Marley, LM Russell, C Ochoa, TB Onasch, MS Pekour, C Song, IM Ulbrich, C Warneke, D Welsh-Bon, C Wiedinmyer, DR Worsnop, X-Y Yu, and RA Zaveri. 2009. "Evaluating Simulated Primary Anthropogenic and Biomass Burning Organic Aerosols During Milagro: Implications for Assessing Treatments of Secondary Organic Aerosols." Atmospheric Chemistry and Physics 9(16):6191-215.

Ferguson, MR, KR Minard, and KM Krishnan. 2009, "Optimization of Nanoparticle Core Size for Magnetic Particle Imaging.” Journal of Magnetism and Magnetic Materials 321(10):1548-51.

Fernandez CA, RJ Wiacek, P Nachimuthu, GE Fryxell, AM Pierson, CL Warner, MG Warner, and RS Addleman. 2008. "A Simple Method for the Prevention of Non-Specific Adsorption by Nanocrystals onto Surfaces." Journal of Nanoscience and Nanotechnology 8(11):5781-5786.

doi:10.1166/jnn.2008.320

Fernandez, CA, JG Bekhazi, EM Hoppes, RJ Wiacek, GE Fryxell, JT Bays, MG Warner, CM Wang, JE Hutchinson, and RS Addleman. 2009. "Advancements toward the Greener Processing of Engineered Nanomaterials -- Effect of Core Size on the Dispersibility and Transport of Gold Nanocrystals in nearCritical Solvents." Small 5(8):961-969. 
Fernandez, CA, JG Roberts, EM Hoppes, GE Fryxell, CM Wang, JT Bays, MG Warner, RJ Wiacek, and RS Addleman. 2009, "Effect of the Ligand Shell Composition on the Dispersibility and Transport of Gold Nanocrystals in near Critical Solvents." Langmuir 25(9):4900-06.

Flaud, J-m, WJ Lafferty, and RL Sams. 2009, "Line Intensities for the V1, V3, and V1 + V3 Bands of 34so2.” Journal of Quantitative Spectroscopy and Radiative Transfer 110(9-10):669-74.

Fox E, S Velu, MH Engelhard, YH Chin, JT Miller, J Kropf, and C Song. 2008. "Characterization of $\mathrm{CeO} 2-$ Supported Cu-Pd Bimetallic Catalyst for the Oxygen-Assisted Water-Gas Shift Reaction." Journal of Catalysis 260(2):358-370. doi:10.1016/j.jcat.2008.08.018

Fredrickson, JK, JM Zachara, AE Plymale, SM Heald, JP McKinley, DW Kennedy, C Liu, and P Nachimuthu. 2009, "Oxidative Dissolution Potential of Biogenic and Abiogenic Tco2 in Subsurface Sediments." Geochimica et Cosmochimica Acta 73(8):962-76.

Gao, F, HY Xiao, XT Zu, M Posselt, and WJ Weber. 2009. "Defect-Enhanced Charge Transfer by IonSolid Interactions in SiC Using Large-Scale Ab Initio Molecular Dynamics Simulations." Physical Review Letters 103(2):Article number: 027405.

Gao, F, WJ Weber, HY Xiao, and XT Zu. 2009. "Formation and Properties of Defects and Small Vacancy Clusters in SiC: Ab Initio Calculations." Nuclear Instruments and Methods in Physics Research. Section B, Beam Interactions with Materials and Atoms 267(18):2995-98.

Gibbs, GV, AF Wallace, DF Cox, PM Dove, RT Downs, NL Ross, and KM Rosso. 2009. "Role of Directed Van Der Waals Bonded Interactions in the Determination of the Structures of Molecular Arsenate Solids." Journal of Physical Chemistry A 113(4):736-749.

Gibbs, GV, AF Wallace, DF Cox, RT Downs, NL Ross, and KM Rosso. 2009. "Bonded Interactions in Silica Polymorphs, Silicates and Siloxane Molecules.” American Mineralogist 94(8-9):1085-102.

Goering J, U Burghaus, BW Arey, O Eidelman, and R Tenne. 2008. "Reactive and non-reactive interactions of thiophene with WS2 fullerene-like nanoparticles: an ultra-high vacuum surface chemistry study ." Catalysis Letters 125(3-4):236-242. doi:10.1007/s10562-008-9565-7

Govind, N, M Valiev, L Jensen, and K Kowalski. 2009, "Excitation Energies of Zinc Porphyrin in Aqueous Solution Using Long-Range Corrected Time-Dependent Density Functional Theory." Journal of Physical Chemistry A 113(21):6041-43.

Govind, N, PV Sushko, WP Hess, M Valiev, and K Kowalski. 2009. "Excitons in Potassium Bromide: A Study Using Embedded Time-Dependent Density Functional Theory and Equation-of-Motion Coupled Cluster Methods." Chemical Physics Letters 470(4-6):353-357.

Grate, JW, MG Warner, RM Ozanich, KD Miller, HA Colburn, BP Dockendorff, KC Antolick, NC Anheier, MA Lind, J Lou, JD Marks, and CJ Bruckner-Lea. 2009. "Renewable Surface Fluorescence Sandwich Immunoassay Biosensor for Rapid Sensitive Botulinum Toxin Detection in an Automated Fluidic Format." Analyst 134(5):987 - 996. 
Grider, G, J Nunez, J Bent, R Ross, L Ward, S Poole, EJ Felix, E Salmon, and M Bancroft. 2009. "Coordinating Government Funding of File System and I/O Research through the High End Computing University Research Activity." Operating Systems Review 43(1):2-7.

Groves JF, Y Du, I Lyubinetsky, and DR Baer. 2008. "Focused Ion Beam Directed Self-Assembly (Cu2O on SrTiO3): FIB Pit and Cu2O Quantum Dot Evolution." Superlattices and Microstructures 44(45):677-685. doi:10.1016/j.spmi.2008.01.016

Gupta, S, SVNT Kuchibhatla, MH Engelhard, V Shutthanandan, P Nachimuthu, W Jiang, LV Saraf, S Thevuthasan, and S Prasad. 2009, "Influence of Samaria Doping on the Resistance of Ceria Thin Films and Its Implications to the Planar Oxygen Sensing Devices." Sensors and Actuators. B, Chemical 139(2):380-86.

Hadjar, O, P Wang, JH Futrell, and J Laskin. 2009, "Effect of the Surface on Charge Reduction and Desorption Kinetics of Soft Landed Peptide Ions." Journal of the American Society for Mass Spectrometry 20(6):901-06.

Hammond, JR, and K Kowalski. 2009, "Parallel Computation of Coupled Cluster Hyperpolarizabilities." Journal of Chemical Physics 130(19):Art. No. 194108.

Harvey, SD, GW Buchko, RB Lucke, CW Wright, AM Melville, AJ Scott, and BW Wright. 2009, "The Structure and Purity of a Reference Dye Standard Used for Quantification of C.I. Solvent Red 164 in Fuels.” Dyes and Pigments 82(3):307-15. 10.1016/j.dyepig.2009.01.015

Heald, SM, TC Kaspar, T Droubay, V Shutthanandan, SA Chambers, A Mokhtari, AJ Behan, HJ Blythe, JR Neal, AM Fox, and GA Gehring. 2009. "X-Ray Absorption Fine Structure and Magnetization Characterization of the Metallic Co Component in Co-Doped ZnO Thin Films." Physical Review. B, Condensed Matter 79(7):Art. No. 075202.

Heibeck, TH, S-J Ding, LK Opresko, R Zhao, AA Schepmoes, F Yang, AV Tolmachev, ME Monroe, DG Camp, RD Smith, HS Wiley, and W Qian. 2009. “An Extensive Survey of Tyrosine Phosphorylation Revealing New Sites in Human Mammary Epithelial Cells.” Journal of Proteome Research 8(8):3852-61.

Henager, CH, DJ Edwards, AL Schemer-Kohrn, M Bliss, and JE Jaffe. 2009, "Preferential Orientation of Te Precipitates in Melt-Grown CZT.” Journal of Crystal Growth 311(9):2641 - 47.

10.1016/j.jcrysgro.2009.03.002

Henderson MA. 2008. "Ethyl Radical Ejection During Photodecomposition of Butanone on TiO2(110).” Surface Science 602(20):3188-3193. doi:10.1016/j.susc.2007.06.079

Henne, KL, CN Nakatsu, DK Thompson, and A Konopka. 2009. "High-Level Chromate Resistance in Arthrobacter Sp. Strain FB24 Requires Previously Uncharacterized Accessory Genes.” BMC Microbiology 9:199-213.

Hess NJ, O Qafoku, Y Xia, and AR Felmy. 2008. "Thermodynamic Model for Solubility of TcO2•xH2O in Aqueous Oxalate System." Journal of Solution Chemistry 37(11):1471-1487. doi:10.1007/s10953008-9328-5 
Hess, NJ, GK Schenter, MR Hartman, LL Daemen, TE Proffen, SM Kathmann, CJ Mundy, MA Hartl, DJ Heldebrant, AC Stowe, and T Autrey. 2009, "Neutron Powder Diffraction and Molecular Simulation Study of the Structural Evolution of Ammonia Borane from 15 to 340 K." Journal of Physical Chemistry A 113(9):5723-35.

Hlaing Oo, WM, LV Saraf, MH Engelhard, V Shutthanandan, L Bergman, J Huso, and MD McCluskey. 2009. "Suppression of Conductivity in Mn-Doped ZnO Thin Films." Journal of Applied Physics 105(1):013715.

Hu D, Z Tian, W Wu, W Wan, and AD Li. 2008. "Photoswitchable Nanoparticles Enable HighResolution Cell Imaging: PULSAR Microscopy." Journal of the American Chemical Society 130(46):15279-15281. doi:10.1021/ja805948u

Hu J, RA Dagle, BR Johnson, HW Kreuzer-Martin, DJ Gaspar, BQ Roberts, and ML Alexander. 2008. "Development of Micropyrolyzer for Enhanced Isotope Ratio Measurement." Industrial and Engineering Chemistry Research 47(22):8625-8630. doi:10.1021/ie8009236

Hu, JZ, JA Sears, JH Kwak, DW Hoyt, Y Wang, and CHF Peden. 2009, “An Isotropic Chemical ShiftChemical Shift Anisotropic Correlation Experiment Using Discrete Magic Angle Turning." Journal of Magnetic Resonance 198(1):105-10.

Hu, JZ, JH Kwak, Y Wang, CHF Peden, H Zheng, D Ma, and X Bao. 2009. "Studies of the Active Sites for Methane Dehydroaromatization Using Ultrahigh-Field Solid-State Mo95 NMR Spectroscopy." Journal of Physical Chemistry C 113(7):2936-2942.

Hu, JZ, JH Kwak, Z Yang, X Wan, and LL Shaw. 2009, "Direct Observation of Ion Exchange in Mechanically Activated Lih+Mgb2 System Using Ultra-High Field Nuclear Magnetic Resonance Spectroscopy.” Applied Physics Letters 94(14):Art. No. 141905. 10.1063/1.3110966

Hu, Q, P Wang, PL Gassman, and J Laskin. 2009. "In Situ Studies of Soft- and Reactive Landing of Mass-Selected Ions Using Infrared Reflection Absorption Spectroscopy." Analytical Chemistry 81(17):7302-08.

Huang, W, and LS Wang. 2009, "Probing the 2D to 3D Structural Transition in Gold Cluster Anions Using Argon Tagging." Physical Review Letters 102(15):153401(4).

Huang, W, S Bulusu, R Pal, XC Zeng, and LS Wang. 2009, "Structural Transition of Gold Nanoclusters: From the Golden Cage to the Golden Pyramid.” ACS Nano 3(5):1225-30. 1021/nn900232d

Ishimaru, M, Y Zhang, and WJ Weber. 2009. "Ion-Beam-Induced Chemical Disorder in GaN." Journal of Applied Physics 106(5):053513, 1-4.

Jehle, S, B van Rossum, JR Stout, SM Noguchi, K Falber, K Rehbein, H Oschkinat, RE Klevit, and P Rajagopal. 2009. “ $\alpha$ B-Crystallin: A Hybrid Solid-State/Solution-State NMR Investigation Reveals Structural Aspects of the Heterogeneous Oligomer." Journal of Molecular Biology 385(5):1482-1497.

Jensen, L, and N Govind. 2009. "Excited States of DNA Base Pairs Using Long-Range Corrected TimeDependent Density Functional Theory." Journal of Physical Chemistry A 113(36):9761-65. 
Jiang, W, and WJ Weber. 2009, "Anisotropy of Disorder Accumulation and Recovery in 6h-Sic Irradiated with Au2+ Ions at 140 K." Journal of Nuclear Materials 389(2):332-35.

Jiang, W, WJ Weber, J Lian, and NM Kalkhoran. 2009. "Disorder Accumulation and Recovery in GoldIon Irradiated 3C-SiC.” Journal of Applied Physics 105(1):Art. No. 013529.

Johnson, BR, BJ Riley, SK Sundaram, JV Crum, CH Henager, Y Zhang, V Shutthanandan, CE Seifert, RM Van Ginhoven, CE Chamberlin, A Rockett, D Hebert, and A Aquino. 2009, "Synthesis and Characterization of Bulk Vitreous Cadmium Germanium Arsenide." Journal of the American Ceramic Society 92(6):1236-43. 10.1111/j.1551-2916.2009.03001.x

Johnson, TJ, RL Sams, SD Burton, and TA Blake. 2009. "Absolute Integrated Intensities of Vapor-Phase Hydrogen Peroxide (H202) in the Mid-Infrared at Atmospheric Pressure." Analytical and Bioanalytical Chemistry 395(2):377-86.

Kelly JF, A Maki, TA Blake, and RL Sams. 2008. "Supersonic Free-jet Quantum Cascade Laser Measurements of v4 for CF3(35)Cl and CF3(37)Cl and FTS Measurements from 400 to $1260 \mathrm{~cm}-1 . "$ Journal of Molecular Spectroscopy 252(1):81-89. doi:10.1016/j.jms.2008.07.001

Kelly, RT, JS Page, I Marginean, K Tang, and RD Smith. 2009. "Dilution-Free Analysis from Picoliter Droplets by Nano-Electrospray Ionization Mass Spectrometry." Angewandte Chemie International Edition 48(37):6832-35.

Kim J, JW Grate, and P Wang. 2008. "Nanobiocatalysis and Its Potential Applications." Trends in Biotechnology 26(11):639-646. doi:10.1016/j.tibtech.2008.07.009

Kim, BC, D Lopez-Ferrer, S-m Lee, H-k Ahn, S Nair, SH Kim, BS Kim, K Petritis, DG Camp, JW Grate, RD Smith, Y-m Koo, MB Gu, and J Kim. 2009, "Highly Stable Trypsin-Aggregate Coatings on Polymer Nanofibers for Repeated Protein Digestion.” Proteomics 9(7):1893-900.

Kim, DH, J Szanyi, JH Kwak, X Wang, JC Hanson, MH Engelhard, and CHF Peden. 2009, "Effects of Sulfation Level on the Desulfation Behavior of Pre-Sulfated Pt BaO/A12O3 Lean NOx Trap Catalysts: A Combined H2 Temperature-Programmed Reaction, in-Situ Sulfur K-Edge X-Ray Absorption near-Edge Spectroscopy, X-Ray Photoelectron Spectroscopy, and Time-Resolved X-Ray Diffraction Study.” Journal of Physical Chemistry C 113(17):7336-41. 10.1021/jp900304h

Kimball, BE, R Mathur, A Dohnalkova, A Wall, R Runkel, and SL Brantley. 2009. "Copper Isotope Fractionation in Acid Mine Drainage." Geochimica et Cosmochimica Acta 73(5):1247-1263.

Kimmel, GA, J Matthiesen, M Baer, CJ Mundy, NG Petrik, RS Smith, Z Dohnalek, and BD Kay. 2009. "No Confinement Needed: Observation of a Metastable Hydrophobic Wetting Two-Layer Ice on Graphene." Journal of the American Chemical Society 131(35):12838-44.

Kirkpatrick, RW, T Masiello, N Jariyasopit, JW Nibler, AG Maki, TA Blake, and A Weber. 2009. "HighResolution Rovibrational Study of the Coriolis-Coupled $\mathrm{Nu}(12)$ and $\mathrm{Nu}(15)$ Modes of [1.1.1]Propellane." Journal of Molecular Spectroscopy 253(1):41-50. 
Kleiner I, N Moazzen-Ahmadi, ARW McKellar, TA Blake, RL Sams, SW Sharpe, G Moruzzi, and JT Hougen. 2008. "Assignment, Fit, and Theoretical Discussion of the v10 Band of Acetaldehyde Near 509 cm-1.” Journal of Molecular Spectroscopy 252(2):214-229. doi:10.1016/j.jms.2008.09.004

Kleinman, LI, SR Springston, JX Wang, PH Daum, YN Lee, LJ Nunnermacker, G Senum, J WeinsteinLloyd, ML Alexander, JM Hubbe, JV Ortega, RA Zaveri, MR Canagaratna, and JT Jayne. 2009. "The Time Evolution of Aerosol Size Distribution over the Mexico City Plateau." Atmospheric Chemistry and Physics 9(13):4261-78.

Kou, R, Y Shao, D Wang, MH Engelhard, JH Kwak, J Wang, VV Viswanathan, CM Wang, Y Lin, Y Wang, IA Aksay, and J Liu. 2009, "Enhanced Activity and Stability of Pt Catalysts on Functionalized Graphene Sheets for Electrocatalytic Oxygen Reduction." Electrochemistry Communications 11(5):95457.

Kowalski K, JR Hammond, WA De Jong, and AJ Sadlej. 2008. "Coupled Cluster Calculations for Static and Dynamic Polarizabilities of $\mathrm{C}_{60}$." Journal of Chemical Physics 129(22):226101.

doi:10.1063/1.3028541

Kowalski, K, and P-D Fan. 2009. "Generating Functionals Based Formulation of the Method of Moments of Coupled Cluster Equations." Journal of Chemical Physics 130(8):084112.

Kowalski, K. 2009, "Nested Variant of the Method of Moments of Coupled Cluster Equations for Vertical Excitation Energies and Excited-State Potential Energy Surfaces.” Journal of Chemical Physics 130(19):Art. No. 194110.

Kozimor, SA, P Yang, ER Batista, KS Boland, CJ Burns, DL Clark, SD Conradson, RL Martin, MP Wikerson, and LE Wolfsberg. 2009. "Trends in Covalency for D- and F-Element Metallocene Dichlorides Identified Using Chlorine K-Edge X-Ray Absorption Spectroscopy and Time Dependent-Density Functional Theory." Journal of the American Chemical Society 131(34):12125-36.

Kroeker, S, I Farnan, S Schuller, and T Advocat. 2009, "95Mo NMR Study of Crystallization in Model Nuclear Waste Glasses." in Scientific Basis for Nuclear Waste Management XXXII, Materials Research Society Proceedings of the Fall Meeting, eds. RB Rebak, NC Hyatt and DA Pickett, Vol 1124, pp. Art. No.: 1124-Q03-06 Plenum Press. 10.1557/PROC-1124-Q03-06

Krupka, KM, M Parkhurst, K Gold, BW Arey, ED Jenson, and RA Guilmette. 2009. "Physicochemical Characterization of Capstone Depleted Uranium Aerosols Iii: Morphologic and Chemical Oxide Analyses.” Health Physics 93(3):276-291.

Kuchibhatla, SVNT, AS Karakoti, DC Sayle, H Heinrich, and S Seal. 2009. "Symmetry-Driven Spontaneous Self-Assembly of Nanoscale Ceria Building Blocks to Fractal Super-Octahedra." Crystal Growth \& Design 9(3):1614-1620.

Kuchibhatla, SVNT, P Nachimuthu, F Gao, W Jiang, V Shutthanandan, MH Engelhard, S Seal, and S Thevuthasan. 2009, "Growth-Rate Induced Epitaxial Orientation of Ceo2 on A12o3(0001)." Applied Physics Letters 94(20):204101:1-3. 10.1063/1.3139073 
Kuchibhatla, SVNT, SY Hu, Z Yu, V Shutthanandan, Y Li, P Nachimuthu, W Jiang, S Thevuthasan, CH Henager, and SK Sundaram. 2009. "Morphology, Orientation Relationship and Stability Analysis of Cu2o Nanoclusters on Srtio3 (100).” Applied Physics Letters 95(5):Art. No. 053111.

Kulik HJ, and NN Marzari. 2008. “A Self-Consistent Hubbard U Density-Functional Theory Approach to the Addition-Elimination Reactions of Hydrocarbons on Bare $\mathrm{FeO}^{+}$." Journal of Chemical Physics 129(13):134314. doi:10.1063/1.2987444

Kwak JH, DH Kim, J Szanyi, and CHF Peden. 2008. "Excellent Sulfur Resistance of Pt/BaO/CeO2 Lean NOx Trap Catalysts.” Applied Catalysis. B, Environmental 84(3-4):545-551.

doi:10.1016/j.apcatb.2008.05.009

Kwak, JH, D Mei, C-WW Yi, DH Kim, CHF Peden, L Allard, and J Szanyi. 2009. "Understanding the Nature of Surface Nitrates in BaO/Gamma-Al2o3 NOx Storage Materials: A Combined Experimental and Theoretical Study." Journal of Catalysis 261(1):17-22.

Kwak, JH, JZ Hu, D Mei, C-WW Yi, DH Kim, CHF Peden, L Allard, and J Szanyi. 2009. "Coordinatively Unsaturated A13+ Centers as Binding Sites for Active Catalyst Phases on $\gamma$-A12O3." Science 325(5948):1670-73.

Lafferty W, J Flaud, RL Sams, and EHA Ngom. 2008. "High Resolution Analysis of the Rotational Levels of the (000), (010), (100), (001), (020), (110) and (011) Vibrational States of 34S 16O2." Journal of Molecular Spectroscopy 252(1):72-76. doi:10.1016/j.jms.2008.06.013

Lafferty, W, J-m Flaud, EHA Ngom, and RL Sams. 2009. “(SO2)-S-34-O-16: High-Resolution Analysis of the (030),(101), (111), (002) and (201) Vibrational States; Determination of Equilibrium Rotational Constants for Sulfur Dioxide and Anharmonic Vibrational Constants." Journal of Molecular Spectroscopy 253(1):51-54.

Laskin, A, JS Smith, and J Laskin. 2009, "Molecular Characterization of Nitrogen Containing Organic Compounds in Biomass Burning Aerosols Using High Resolution Mass Spectrometry." Environmental Science \& Technology 43(10):3764-71. 10.1021/es803456n

Leavitt, CM, VS Bryantsev, WA De Jong, MS Diallo, WA Goddard Iii, GS Groenewold, and MJ Van Stipdonk. 2009. "Addition of $\mathrm{H} 2 \mathrm{O}$ and O-2 to Acetone and Dimethylsulfoxide Ligated Uranyl(V) Dioxocations." Journal of Physical Chemistry A 113(11):2350-2358.

Lee AC, Z Dai, B Chen, H Wu, J Wang, A Zhang, L Zhang, TM Lim, and Y Lin. 2008. "Electrochemical Branched-DNA Assay for Polymerase Chain Reaction-Free Detection and Quantification of Oncogenes in Messenger RNA.” Analytical Chemistry 80(24):9402-9410. doi:10.1021/ac801263r

Lei C, D Hu, and EJ Ackerman. 2008. "Single-molecule Fluorescence Spectroelectrochemistry of Cresyl Violet.” Chemical Communications (43):5490-5492. doi:10.1039/b812161c

Lei, C, D Hu, and EJ Ackerman. 2009. "Clay Nanoparticle-Supported Single-Molecule Fluorescence Spectroelectrochemistry." Nano Letters 9(2):655-658. 
Li Y, L Ma, X Zhang, AG Joly, Z Liu, and W Chen. 2008. "Synthesis and Optical Properties of Sulfide Nanoparticles Prepared in Dimethylsulfoxide." Journal of Nanoscience and Nanotechnology 8(11):56465651.

Li, J, and Y Lin. 2009, "Nanomaterials for Sensing and Electrocatalysis.” Journal of Nanoscience and Nanotechnology 9(4):2173-74.

Lian, J, J Zhang, F Namavar, Y Zhang, F Lu, H Haider, K Garvin, WJ Weber, and RC Ewing. 2009, "Ion Beam-Induced Amorphous-to-Tetragonal Phase Transformation and Grain Growth of Nanocrystalline Zirconia." Nanotechnology 20(24):245303, 1-7.

Liang B, JC Lehmann, D Solomon, S Sohi, JE Thies, JO Skjemstad, FJ Luizao, MH Engelhard, EG Neves, and S Wirick. 2008. "Stability of Biomass-derived Black Carbon in Soils ." Geochimica et Cosmochimica Acta 72(24):6069-6078. doi:10.1016/j.gca.2008.09.028

Lim IIS, D Mott, MH Engelhard, Y Pan, S Kamodia, J Luo, P Njoki, S Zhou, and CJ Zhong. 2008. "Interparticle Chiral Recognition of Enantiomers: A Nanoparticle-Based Regulation Strategy." Analytical Chemistry 2009, 81(2):689-698. doi:10.1021/ac802119p

Lim, IIS, D Mott, MH Engelhard, Y Pan, S Kamodia, J Luo, P Njoki, S Zhou, L Wang, and C-J Zhong. 2009. "Interparticle Chiral Recognition of Enantiomers: A Nanoparticle-Based Regulation Strategy." Analytical Chemistry 81(2):689-698.

Lin, Y, and HS Nalwa. 2009. Handbook of Electrochemical Nanotechnology. American Scientific Publishers, Stevenson Ranch, CA.

Lin, Y, and X Cui. 2009. "Nanocarbon-Based Nanocatalysts: Synthesis and Applications in Fuel Cells." Chapter 7 in Handbook of Electrochemical Nanotechnology. ed. Y Lin and H Nalwa, Vol I, pp. 145-164. American Scientific Publishers, Stevenson Ranch, CA.

Lin, Y, D Choi, J Wang, and JR Bontha. 2009. "Nanomaterials-Enhanced Electrically Switched Ion Exchange Process for Water Treatment." Chapter 14 in Nanotechnology Applications for Clean Water. ed. N Savage et al, pp. 179-189. William Andrew, Norwich, NY.

Liu G, J Wang, RC Barry, CE Petersen, C Timchalk, PL Gassman, and Y Lin. 2008. "NanoparticleBased Electrochemical Immunosensor for the Detection of Phosphorylated Acetylcholinesterase Adduct: An Exposure Biomarker of Organophosphate Pesticides and Nerve Agents." Chemistry - a European Journal 14(32):9951-9959. doi:10.1002/chem.200800412

Liu, C, JM Zachara, L Zhong, SM Heald, Z Wang, BH Jeon, and JK Fredrickson. 2009. "Microbial Reduction of Intragrain U(VI) in Contaminated Sediment." Environmental Science \& Technology 43(13):4928-33.

Liu, C, Z Shi, and JM Zachara. 2009. "Kinetics of Uranium(Vi) Desorption from Contaminated Sediments: Effect of Geochemical Conditions and Model Evaluation." Environmental Science \& Technology 43(17):6560-66. 
Liu, Y, and A Laskin. 2009. "Hygroscopic Properties of CH3SO3Na, CH3SO3NH4, (CH3SO3)2Mg and (CH3SO3)2Ca Particles Studied by Micro-FTIR Spectroscopy." Journal of Physical Chemistry A 113(8):1531-1538.

Lopez-Ferrer, D, KK Hixson, HS Smallwood, TC Squier, K Petritis, and RD Smith. 2009. "Evaluation of a High Intensity Focused Ultrasound-Immobilized Trypsin Digestion and 18 O-Labeling Method for Quantitative Proteomics." Analytical Chemistry 81(15):6272-77.

Lower, BH, R Yongsunthon, L Shi, L Wildling, HJ Gruber, NS Wigginton, CL Reardon, GE Pinchuk, T Droubay, JF Boily, and SK Lower. 2009, “Antibody Recognition Force Microscopy Shows That Outer Membrane Cytochromes OmcA and MtrC Are Expressed on the Exterior Surface of Shewanella Oneidensis MR-1.” Applied and Environmental Microbiology 75(9):2931-35.

Lu CY, PJ Shamberger, EN Yitamben, KM Beck, AG Joly, MA Olmstead, and FS Ohuchi. 2008. "Laser and Electrical Current Induced Phase Transformation of In2Se3 Semiconductor thin film on Si(111) ." Applied Physics A, Materials Science and Processing 93(1):93-98. doi:10.1007/s00339-008-4776-8

Ma Ham, A, Z Tang, H Wu, J Wang, and Y Lin. 2009. "Protein-Based Nanomedicine Platforms for Drug Delivery." Small 5(15):1706-21.

Mabrouki, RB, RT Kelly, DC Prior, AA Shvartsburg, K Tang, and RD Smith. 2009. "Improving Faims Sensitivity Using a Planar Geometry with Slit Interfaces." Journal of the American Society for Mass Spectrometry 20(9):1768-74.

Maki, A, T Masiello, TA Blake, JW Nibler, and A Weber. 2009, "On the Determination of C0 (or A0), D0K, H0K, and Some Dark States for Symmetric-Top Molecules from Infrared Spectra without the Need for Localized Perturbations.” Journal of Molecular Spectroscopy 255(1):56-62.

Marginean, I, RT Kelly, RJ Moore, DC Prior, BL Lamarche, K Tang, and RD Smith. 2009, "Selection of the Optimum Electrospray Voltage for Gradient Elution LC-MS Measurements." Journal of the American Society for Mass Spectrometry 20(4):682-88.

Marshall, MJ, A Dohnalkova, DW Kennedy, AE Plymale, SH Thomas, FE Loffler, R Sanford, JM Zachara, JK Fredrickson, and AS Beliaev. 2009. "Electron Donor-Dependent Radionuclide Reduction and Nanoparticle Formation by Anaeromyxobacter Dehalogenans Strain 2CP-C." Environmental Microbiology 11(2):534-543.

Masiello, T, A Maki, and TA Blake. 2009, "Analysis of the High-Resolution Infrared Spectrum of Cyclopropane.” Journal of Molecular Spectroscopy 255(1):45-55.

Meakin P, and KM Rosso. 2008. "Simple Kinetic Monte Carlo Models for Dissolution Pitting Induced by Crystal Defects.” Journal of Chemical Physics 129(20):204106. doi:10.1063/1.3021478

Mei D, Q Ge, JH Kwak, DH Kim, J Szanyi, and CHF Peden. 2008. “Adsorption and Formation of BaO Overlayers on Gamma-A12O3 Surfaces ." Journal of Physical Chemistry C 112(46):18050-18060. doi:10.1021/jp806212z 
Mei, D, L Xu, and GA Henkelman. 2009. "Potential Energy Surface of Methanol Decomposition on $\mathrm{Cu}(110)$." Journal of Physical Chemistry C 113(11):4522-4537.

Mei, D, Q Ge, J Szanyi, and CHF Peden. 2009, "First-Principles Analysis of NOx Adsorption on Anhydrous $\gamma$-A12O3 Surfaces.” Journal of Physical Chemistry C 113(18):7779-89. 10.1021/jp8103563

Minard KR, RE Jacob, G Laicher, DR Einstein, AP Kuprat, and RA Corley. 2008. "MR Imaging of Apparent $3 \mathrm{He}$ Gas Transport in Narrow Pipes and Rodent Airways ." Journal of Magnetic Resonance 194(2):182-191. doi:10.1016/j.jmr.2008.07.006

Minato, T, Y Sainoo, Y Kim, HS Kato, K-i Aika, M Kawai, J Zhao, H Petek, T Huang, W He, B Wang, Z Wang, Y Zhao, J Yang, and JG Hou. 2009. "The Electronic Structure of Oxygen Atom Vacancy and Hydroxyl Impurity Defects on Titanium Dioxide (110) Surface." Journal of Chemical Physics 130(12):124502-124501 - 124502-124511.

Moffet RC, Y Desyaterik, RJ Hopkins, AV Tivanski, MK Gilles, YA Wang, V Shutthanandan, LT Molina, R Gonzalez, KS Johnson, V Mugica, MJ Molina, A Laskin, and KA Prather. 2008. "Characterization of Aerosols Containing $\mathrm{Zn}, \mathrm{Pb}$, and $\mathrm{Cl}$ from an Industrial Region of Mexico City." Environmental Science \& Technology 42(19):7091-7097. doi:10.1021/es7030483

Mudiyanselage, K, C-W Yi, and J Szanyi. 2009, "Oxygen Coverage Dependence of NO Oxidation on Pt(111).”Journal of Physical Chemistry C 113(14):5766-76.

Mudiyanselage, K, C-WW Yi, and J Szanyi. 2009. "Reactivity of a Thick BaO Film Supported on $\mathrm{Pt}(111)$ : Adsorption and Reaction of NO2, H2O and CO2." Langmuir 25(18):10820-28.

Murugesan, V, SN Kerisit, CM Wang, Z Nie, KM Rosso, Z Yang, GL Graff, J Liu, and JZ Hu. 2009. "Effect of Chemical Lithium Intercalation into Rutile TiO2 Nanorods." Journal of Physical Chemistry C 113(32):14567-74.

Myler, PJ, R Stacy, LJ Stewart, BL Staker, WC Van Voorhis, G Varani, and GW Buchko. 2009. "The Seattle Structure Genomics Center for Infectious Disease (Ssgcid)." Infectious Disorders Drug Targets 9(5):493-506.

Nachimuthu, P, YJ Kim, SVNT Kuchibhatla, Z Yu, W Jiang, MH Engelhard, V Shutthanandan, J Szanyi, and S Thevuthasan. 2009. "Growth and Characterization of Barium Oxide Nanoclusters on Ysz(111)." Journal of Physical Chemistry C 113(32):14324-28.

Neiner, D, AJ Karkamkar, JC Linehan, BW Arey, T Autrey, and SM Kauzlarich. 2009. "Promotion of Hydrogen Release from Ammonia Borane with Mechanically Activated Hexagonal Boron Nitride." Journal of Physical Chemistry C 113(3):1098-1103.

Nellis, S, H Yoon, C Werth, M Oostrom, and AJ Valocchi. 2009, "Surface and Interfacial Properties of Nonaqueous-Phase Liquid Mixtures Released to the Subsurface at the Hanford Site." Vadose Zone Journal 8(2):344-51. 
Nichols, PJ, N Govind, EJ Bylaska, and WA De Jong. 2009. "Gaussian Basis Set and Planewave Relativistic Spin-Orbit Methods in NWChem." Journal of Chemical Theory and Computation 5(3):491499.

Nie JL, H Xiao, XT Zu, and F Gao. 2008. "Hydrogen Adsorption, Dissociation and Diffusion on the $\alpha$ U(001) Surface.” Journal of Physics. Condensed matter 20(44):445001. doi:10.1088/0953$8984 / 20 / 44 / 445001$

O'Hara, MJ, SR Burge, and JW Grate. 2009. "Quantification of Technetium-99 in Complex Groundwater Matrixes Using a Radiometric Preconcentrating Minicolumn Sensor in an Equilibration-Based Sensing Approach." Analytical Chemistry 81(3):1068-1078.

Ohsawa T, I Lyubinetsky, MA Henderson, and SA Chambers. 2008. "Hole-mediated Photodecomposition of Trimehtyl Acetate on a TiO2(001) Anatase Epitaxial Thin Film Surface." Journal of Physical Chemistry C 112(50):20050-20056. doi:10.1021/jp8077997

Ohsawa, T, I Lyubinetsky, Y Du, MA Henderson, V Shutthanandan, and SA Chambers. 2009. "Crystallographic Dependence of Visible-Light Photochemistry in Epitaxial TiO2-xNx Anatase and Rutile.” Physical Review. B, Condensed Matter and Materials Physics 79(8):Art. No. 085401.

Olson A, OM Hyyti, GA Cohen, XH Ning, M Sadilek, NG Isern, and MA Portman. 2008. "Superior Cardiac Function Via Anaplerotic Pyruvate in the Immature Swine Heart After Cardiopulmonary Bypass and Reperfusion." American Journal of Physiology. Heart and Circulatory Physiology 295(6):H2315H2320. doi:10.1152/ajpheart.00739.2008

Oostrom, M, TW Wietsma, JH Dane, MJ Truex, and AL Ward. 2009. "Desiccation of Unsaturated Porous Media: Intermediate-Scale Experiments and Numerical Simulation.” Vadose Zone Journal 8(3):643-50.

Osborn, W, T Markmaitree, LL Shaw, R Ren, JZ Hu, JH Kwak, and Z Yang. 2009, "Solid-State Hydrogen Storage: Storage Capacity,Thermodynamics and Kinetics." JOM. The Journal of the Minerals, Metals and Materials Society 61(4):45-51.

Ozanich, RM, CJ Bruckner-Lea, MG Warner, KD Miller, KC Antolick, JD Marks, J Lou, and JW Grate. 2009. "Rapid Multiplexed Flow Cytometric Assay for Botulinum Neurotoxin Detection Using an Automated Fluidic Microbead-Trapping Flow Cell for Enhanced Sensitivity." Analytical Chemistry 81(14):5783-93.

Parkinson, GS, Z Dohnalek, RS Smith, and BD Kay. 2009. "Reactivity of Fe-0 Atoms, Clusters, and Nanoparticles with CC14 Multilayers on FeO(111)." Journal of Physical Chemistry C 113(5):1818-1829.

Peretyazhko, T, JM Zachara, JF Boily, Y Xia, PL Gassman, BW Arey, and WD Burgos. 2009, "Mineralogical Transformations Controlling Acid Mine Drainage Chemistry." Chemical Geology 262(34):169-78.

Perez-Bergqu, AG, K Sun, Y Zhang, and LM Wang. 2009. "Formation of Gasb Core-Shell Nanofibers by a Thermally-Induced Phase Decomposition Process." Journal of Materials Research 24(7):2286-92. 
Petrik, NG, and GA Kimmel. 2009. "Nonthermal Water Splitting on Rutile TiO2: Electron-Stimulated Production of H-2 and O-2 in Amorphous Solid Water Films on TiO2(110)." Journal of Physical Chemistry C 113(11):4451-4460.

Petrik, NG, Z Zhang, Y Du, Z Dohnalek, I Lyubinetsky, and GA Kimmel. 2009. "Chemical Reactivity of Reduced TiO2(110): The Dominant Role of Surface Defects in Oxygen Chemisorption.” Journal of Physical Chemistry C 113(28):12407-11.

Priyantha, WA, RJ Smith, H Chen, M Kopczyk, M Lerch, C Key, P Nachimuthu, and W Jiang. 2009. "Fe-Al Interface Intermixing and the Role of Ti, V, and $\mathrm{Zr}$ as a Stabilizing Interlayer at the Interface." Journal of Applied Physics 105(5):Art. No. 053504.

Qafoku, N, L Zhong, CJ Thompson, C Liu, BW Arey, AV Mitroshkov, and RG Riley. 2009. "Physical Control on $\mathrm{CCl} 4$ and $\mathrm{CHCl} 3$ Desorption from Artificially Contaminated and Aged Sediments with Supercritical Carbon Dioxide." Chemosphere 74(4):494-500.

Qian W, DT Kaleta, BA Ogata, H Jiang, T Liu, X Zhang, HM Mottaz, SM Varnum, DG Camp, II, huang, X Fang, W Zhang, and RD Smith. 2008. "Enhanced Detection of Low-Abundant Human Plasma Proteins using a Tandem IgY12-SuperMix Immunoaffinity Separation Strategy." Molecular \& Cellular Proteomics. MCP 7(10):1963-1973. doi:10.1074/mcp.M800008-MCP200

Qian, W, T Liu, VA Petyuk, MA Gritsenko, BO Petritis, AD Polpitiya, A Kaushal, W Xiao, CC Finnerty, MG Jescheke, N Jaitly, ME Monroe, RJ Moore, LL Moldawer, RW Davis, RG Tompkins, DN Hemdon, DG Camp, and RD Smith. 2009. "Large-Scale Multiplexed Quantitative Discovery Proteomics Enabled by the Use of an O-18-Labeled 'Universal' Reference Sample." Journal of Proteome Research 8(1):290299.

Rai D, M Yui, DA Moore, GJ Lumetta, KM Rosso, Y Xia, AR Felmy, and FN Skomurski. 2008. "Thermodynamic Model for ThO2(am) Solubility in Alkaline Silica Solutions ." Journal of Solution Chemistry 37(12):1725-1746. doi:10.1007/s10953-008-9344-5

Ream, TS, JR Haag, AT Wierzbicki, CD Nicora, AD Norbeck, JK Zhu, G Hagen, TJ Guilfoyle, L PasaTolic, and CS Pikaard. 2009. "Subunit Compositions of the RNA-Silencing Enzymes Pol IV and Pol V Reveal Their Origins as Specialized Forms of RNA Polymerase II." Molecular Cell 33(2):192-203.

Rezac J, and P Hobza. 2008. "Benzene Dimer: Dynamic Structure and Thermodynamics Derived from On-the-Fly ab initio DFT-D Molecular Dynamic Simulations." Journal of Chemical Theory and Computation 4(11):1835-1840. doi:10.1021/ct8002282

Rinsland CP, VM Devi, TA Blake, RL Sams, SW Sharpe, and L Chiou. 2008. "Quantitative Measurement of Integrated Band Intensities of Benzene Vapor in the Mid-Infrared at 278, 298, and 323 K." Journal of Quantitative Spectroscopy and Radiative Transfer 109(15):2511-2522.

Rodland KD, JN Adkins, CK Ansong, SM Chowdhury, NP Manes, L Shi, H Yoon, RD Smith, and F Heffron. 2008. "Use of high-throughput mass spectrometry to elucidate host pathogen interactions in Salmonella." Future Microbiology 3(6):625-634. 
Rogers S, M Girolami, W Kolch, KM Waters, T Liu, BD Thrall, and HS Wiley. 2008. "Investigating the Correspondence Between Transcriptomic and Proteomic Expression Profiles Using Coupled Cluster Models." Bioinformatics 24(24):2894-2900. doi:10.1093/bioinformatics/btn553

Rousseau, RJ, GK Schenter, JL Fulton, JC Linehan, MH Engelhard, and T Autrey. 2009. “Defining Active Catalyst Structure and Reaction Pathways from Ab Initio Molecular Dynamics and Operando Xafs: Dehydrogenation of Dimethylaminoborane by Rhodium Clusters." Journal of the American Chemical Society 131(30):10516-24.

Saraf, LV, Z Zhu, CM Wang, and MH Engelhard. 2009. "Microstructure and Secondary Phase Segregation Correlation in Epitaxial/Oriented ZnO Films with Unfavorable Cr Dopant." Journal of Materials Research 24(2):506-515.

Shao Y, J Liu, Y Wang, and Y Lin. 2008. "Novel Catalyst Support Materials for PEM Fuel Cells: Current Status and Future Prospects." Journal of Materials Chemistry 19(1):46-59.

doi: $10.1039 / \mathrm{b} 808370 \mathrm{c}$

Shao, Y, J Wang, R Kou, MH Engelhard, J Liu, Y Wang, and Y Lin. 2009. "The Corrosion of Pem Fuel Cell Catalyst Supports and Its Implications for Developing Durable Catalysts." Electrochimica Acta 54(3109-3114.

Shao, Y, R Kou, J Wang, CM Wang, VV Vishwanathan, J Liu, Y Wang, and Y Lin. 2009. "The Durability Dependence of Pt/CNT Electrocatalysts on the Nanostructures of Carbon Nanotubes: Hollowand Bamboo-Cnts." Journal of Nanoscience and Nanotechnology 9(10):5811-15.

Sharp, JL, JJ Borkowski, DA Schmoyer, DS Daly, SO Purvine, WR Cannon, and GB Hurst. 2009. "Statistically Appraising Process Quality of Affinity-Isolation Experiments." Computational Statistics and Data Analysis 53(5):1720-1726.

She, X, M Flytzani-Stephanopoulos, CM Wang, Y Wang, and CHF Peden. 2009, "SO2-Induced Stability of Ag-Alumina Catalysts in the SCR of NO with Methane." Applied Catalysis. B, Environmental 88(12):98-105.

Shen Y, N Tolic, KK Hixson, SO Purvine, GA Anderson, and RD Smith. 2008. "De novo sequencing of unique sequence tags for discovery of post-translational modifications of proteins ." Analytical Chemistry 80(20):7742-7754. doi:10.1021/ac801123p

Shi, L, SM Chowdhury, HS Smallwood, H Yoon, HM Mottaz-Brewer, AD Norbeck, JE McDermott, TRW Clauss, F Heffron, RD Smith, and JN Adkins. 2009. "Proteomic Investigation of the Time Course Responses of Raw 264.7 Macrophages to Infection with Salmonella Enterica." Infection and Immunity 77(8):3227-33.

Shin, Y, CM Wang, MH Engelhard, and GE Fryxell. 2009. "A Novel Low-Temperature Dendritic Cyclotrimerization of 2,6-Diacetyl Pyridine Leading to Mesoporous Carbon Containing Pyridine Rings." Microporous and Mesoporous Materials 123(1-3):345-48. 
Shin, Y, JY Kim, CM Wang, JF Bonnett, and KS Weil. 2009. "Controlled Deposition of Covalently Bonded Tantalum Oxide on Carbon Supports by Solvent Evaporation Sol-Gel Process." Surface Science 603(15):2290-93.

Shvartsburg AA, and RD Smith. 2008. "Fundamentals of Traveling Wave Ion Mobility Spectrometry." Analytical Chemistry 80(24):9689-9699. doi:10.1021/ac8016295

Shvartsburg, AA, RD Smith, A Wilks, A Koehl, D Ruiz-Alonso, and B Boyle. 2009. "Ultrafast Differential Ion Mobility Spectrometry at Extreme Electric Fields in Multichannel Microchips." Analytical Chemistry 81(15):6489-95.

Singer, DM, JM Zachara, and GE Brown. 2009. "Uranium Speciation as a Function of Depth in Contaminated Hanford Sediments - a Micro-XRF, Micro-XRD, and Micro- and Bulk-XAFS Study." Environmental Science \& Technology 43(3):630-636.

Siu, C-K, J Zhao, J Laskin, IK Chu, AC Hopkinson, and KWM Siu. 2009, "Kinetics for Tautomerizations and Dissociations of Triglycine Radical Cations." Journal of the American Society for Mass Spectrometry 20(6):996-1005.

Smith, JS, A Laskin, and J Laskin. 2009. "Molecular Characterization of Biomass Burning Aerosols Using High Resolution Mass Spectrometry.” Analytical Chemistry 81(4):1512-1521.

Smith, RS, T Zubkov, Z Dohnalek, and BD Kay. 2009. "The Effect of the Incident Collision Energy on the Porosity of Vapor Deposited Amorphous Solid Water Films." Journal of Physical Chemistry B 113(13):4000-4007.

Smith, SC, M Douglas, DA Moore, RK Kukkadapu, and BW Arey. 2009. "Uranium Extraction from Laboratory Synthesized, Uranium-Doped Hydrous Ferric Oxides." Environmental Science \& Technology 43(2341-2347.

Song, T, C Lam, DC Ng, G Orlova, J Laskin, D-C Fang, and IK Chu. 2009, "Experimental and Computational Studies of the Macrocyclic Effect of an Auxiliary Ligand on Electron and Proton Transfers within Ternary Copper(II)-Histidine Complexes." Journal of the American Society for Mass Spectrometry 20(6):972-84.

Sowell, SM, L Wilhelm, AD Norbeck, MS Lipton, CD Nicora, DF Barofsky, C carlson, RD Smith, and SJ Giovannoni. 2009. "Transport Functions Dominate the SAR11 Metaproteome at Low-Nutrient Extremes in the Sargasso Sea." The ISME Journal 3(1):93-105.

Spicer, CW, MW Holdren, KA Cowen, DW Joseph, JR Satola, BP Goodwin, H Mayfield, A Laskin, ML Alexander, JV Ortega, MK Newburn, RH Kagann, and RA Hashmonay. 2009. "Rapid Measurement of Emissions from Military Aircraft Turbine Engines by Downstream Extractive Sampling of Aircraft on the Ground: Results for C-130 and F-15 Aircraft." Atmospheric Environment 43(16):2612-2622.

Spraggins, JM, JA Lloyd, MV Johnston, J Laskin, and DP Ridge. 2009. "Fragmentation Mechanisms of Oxidized Peptides Elucidated by SID, RRKM Modeling and Molecular Dynamics." Journal of the American Chemical Society 20(9):1579-92. 
Springer, DL, JH Miller, SL Spinelli, L Pasa-Tolic, SO Purvine, DS Daly, RC Zangar, S Jin, N Blumberg, CW Francis, MB Taubman, AE Casey, SD Wittlin, and RP Phipps. 2009, "Platelet Proteome Changes Associated with Diabetes and During Platelet Storage for Transfusion." Journal of Proteome Research $8(5): 2261-72$.

Strmcnik DS, DV Tripkovic, D van der Vliet, KC Chang, V Komanicky, H You, G Karapetrov, JP Greeley, VR Stamenkovic, and NM Markovic. 2008. "Unique Activity of Platinum Adislands in the CO Electrooxidation Reaction." Journal of the American Chemical Society 130(46):15332-15339. doi:10.1021/ja8032185

Sun, Z-M, Y-F Zhao, J Li, and LS Wang. 2009. "Diversity of Functionalized Germanium Zintl Clusters: Syntheses and Theoretical Studies of [Ge9PdPPh3]3- and [Ni@(Ge9PdPPh3)]2." Journal of Cluster Science 20(3):601-09.

Tarasevich, BJ, AS Lea, W Bernt, MH Engelhard, and WJ Shaw. 2009. "Adsorption of Amelogenin onto Self-Assembled and Fluoroapatite Surfaces.” Journal of Physical Chemistry B 113(7):1833-1842.

Tartakovsky AM, DM Tartakovsky, TD Scheibe, and P Meakin. 2008. "Hybrid Simulations of Reaction-Diffusion Systems in Porous Media.” SIAM Journal on Scientific Computing 30(6):2799-2816.

Thevuthasan, S, LV Saraf, OA Marina, CM Wang, V Shutthanandan, and PE Burrows. 2009, "Nanoscale Effects on Oxygen Ion Conductance in Oxide Electrolytes." in Handbook of Electrochemical Nanotechnology, eds. Y Lin and H Nalwa. American Scientific Publishers, Los Angeles, CA.

Thonhauser, T, D Ceresoli, AA Mostofi, NN Marzari, R Resta, and D Vanderbilt. 2009. "A Converse Approach to the Calculation of NMR Shielding Tensors." Journal of Chemical Physics 131(10):Article No.: 101101.

Thonhauser, T, D Ceresoli, and NN Marzari. 2009. "NMR Shifts for Polycyclic Aromatic Hydrocarbons from First-Principles.” International Journal of Quantum Chemistry 109(14):3336-42.

Thorn, KA, and LG Cox. 2009. "N-15 NMR Spectra of Naturally Abundant Nitrogen in Soil and Aquatic Natural Organic Matter Samples of the International Humic Substances Society.” Organic Geochemistry 40(4):484-499.

Tian, J, PK Thallapally, SJ Dalgarno, BP McGrail, and JL Atwood. 2009. “Amorphous Molecular Organic Solids for Gas Adsorption.” Angewandte Chemie International Edition 48(30):5492-95.

Toepel, J, JE McDermott, T Summerfield, and LA Sherman. 2009, "Transcriptional Analysis of the Unicellular, Diazotrophic Cyanobacterium Cyanothece Sp. ATCC 51142 Grown under Short Day/Night Cycles." Journal of Phycology 45(3):610-20.

Tolmachev AV, ME Monroe, SO Purvine, RJ Moore, N Jaitly, JN Adkins, GA Anderson, and RD Smith. 2008. "Characterization of Strategies for Obtaining Confident Identifications in Bottom-Up Proteomics Measurements Using Hybrid FTMS instruments ." Analytical Chemistry 80(22):8514-8525. doi:10.1021/ac801376g 
Trevisanutto, PE, PV Sushko, KM Beck, AG Joly, WP Hess, and AL Shluger. 2009. "Excitation, Ionization, and Desorption: How Sub-Band Gap Photons Modify the Structure of Oxide Nanoparticles." Journal of Physical Chemistry C 113(4):1274-1279.

Tsuchiya M, V Shutthanandan, MH Engelhard, and S Ramanathan. 2008. "Direct Measurement of Oxygen Incorporation into Thin Film Oxides at Room Temperature Upon Ultraviolet Phton Irradiation." Applied Physics Letters 93(26):263109. doi:10.1063/1.3058691

Umar, AN, H Kang, AM Timmermans, MP Look, ME Meijer-van Gelder, MA den Bakker, N Jaitly, JW Martens, TM Luider, JA Foekens, and L Pasa-Tolic. 2009, "Identification of a Putative Protein Profile Associating with Tamoxifen Therapy Resistance in Breast Cancer." Molecular \& Cellular Proteomics. MCP 8(6):1278-94. 10.1074/mcp.M800493-MCP200

Valiev, M, R D'Auria, DJ Tobias, and BC Garrett. 2009. "Interactions of Cl- and OH Radical in Aqueous Solution." Journal of Physical Chemistry A 113(31):8823-25.

Villa, O, S Krishnamoorthy, J Nieplocha, and DML Brown. 2009, "Scalable Transparent CheckpointRestart of Global Address Space Applications on Virtual Machines over Infiniband." in Proceedings of the 6th ACM Conference on Computing Frontiers, pp. 197-206. Association for Computing Machinery, New York, NY. 10.1145/1531743.1531776

Vugmeyster, L, D Ostrovsky, JJ Ford, SD Burton, AS Lipton, G Hoatson, and RL Vold. 2009. "Probing the Dynamics of a Protein Hydrophobic Core by Deutron Solid-State Nuclear Magnetic Resonance Spectroscopy." Journal of the American Chemical Society 131(38):13651-58.

Wan, J, Y Kim, TK Tokunaga, Z Wang, S Dixit, CI Steefel, E Saiz, M Kunz, and N Tamura. 2009, "Spatially Resolved U(VI) Partitioning and Speciation: Implications for Plume Scale Behavior of Contaminant U in the Hanford Vadose Zone.” Environmental Science \& Technology 43(7):2247-53.

Wang CM, LV Saraf, and Y Qiang. 2008. "Microstructures of ZnO films deposited on (0001) and r-cut a-A12O3 using MOCVD.” Thin Solid Films 516(23):8337-8342. doi:10.1016/j.tsf.2008.04.001

Wang H, J Wang, C Timchalk, and Y Lin. 2008. "Magnetic Electrochemical Immunoassays with Quantum Dot Labels for Detection of Phosphorylated Acetylcholinesterase in Plasma." Analytical Chemistry 80(22):8477-8484. doi:10.1021/ac801211s

Wang S, X Zhang, X Mao, Q Zeng, H Xu, Y Lin, W Chen, and G Liu. 2008. "Electrochemical Immunoassay of Carcinoembryonic Antigen Based on A Lead Sulfide Nanoparticle Label." Nanotechnology 19(43):Art. No. 435501. doi:10.1088/0957-4484/19/43/435501

Wang XB, HJ Zhai, X Huang, and LS Wang. 2008. "On The Electronic Structure and Chemical Bonding in the Tantalum Trimer Cluster." Journal of Physical Chemistry A 112(43):10962-10967. doi:10.1021/jp806166h

Wang Z, C Liu, X Wang, MJ Marshall, JM Zachara, KM Rosso, M Dupuis, JK Fredrickson, SM Heald, and L Shi. 2008. "Kinetics of Reduction of Fe(III) Complexes by Outer Membrane Cytochromes MtrC and OmcA of Shewanella oneidensis MR-1." Applied and Environmental Microbiology 74(21):67466755. doi:10.1128/AEM.01454-08 
Wang Z, JM Zachara, C Liu, PL Gassman, AR Felmy, and SB Clark. 2008. "A cryogenic fluorescence spectroscopic study of uranyl carbonate, phosphate, and oxyhydroxide minerals." Radiochimica Acta 96(9-11):591-598. doi:10.1524/ract.2008.1541

Wang Z, KB Wagnon, CC Ainsworth, C Liu, KM Rosso, and JK Fredrickson. 2008. "A Spectroscopic Study of the effect of Ligand Complexation on the Reduction of Uranium(VI) by Anthraquinone-2,6disulfonate (AH2DS).” Radiochimica Acta 96(9-11):599-605. doi:10.1524/ract.2008.1542

Wang Z, XT Zu, F Gao, and WJ Weber. 2008. "Nanomechanical Behavior of Single Crystalline SiC Nanotubes Revealed by Molecular Dynamics Simulations." Journal of Applied Physics 104(9):093506. doi:10.1063/1.3005979

Wang, CM, DR Baer, JE Amonette, MH Engelhard, J Antony, and Y Qiang. 2009, "Morphology and Electronic Structure of the Oxide Shell on the Surface of Iron Nanoparticles." Journal of the American Chemical Society 131(25):8824-32. 10.1021/ja900353f

Wang, CM, Z Yang, S Thevuthasan, J Liu, DR Baer, D Choi, D Wang, J Zhang, LV Saraf, and Z Nie. 2009, "Crystal and Electronic Structure of Lithiated Nanosized Rutile TiO2 by Electron Diffraction and Electron Energy-Loss Spectroscopy.” Applied Physics Letters 94(23):Art No. 10.1063/1.3152783

Wang, D, D Choi, J Li, Z Yang, Z Nie, R Kou, D Hu, CM Wang, LV Saraf, J Zhang, IA Aksay, and J Liu. 2009, "Self-Assembled TiO2-Graphene Hybrid Nanostructures for Enhanced Li-Ion Insertion." ACS Nano 3(4):907-14.

Wang, H, D Wingett, MH Engelhard, K Feris, KM Reddy, P Turner, J Layne, C Hanley, J Bell, D Tenne, CM Wang, and A Punnoose. 2009. "Fluorescent Dye Encapsulated ZnO Particles with Cell-Specific Toxicity for Potential Use in Biomedical Applications." Journal of Materials Science. Materials in Medicine 20(1):11-22.

Wang, H, J Wang, D Choi, Z Tang, H Wu, and Y Lin. 2009. "EQCM Immunoassay for Phosphorylated Acetylcholinesterase as a Biomarker for Organophosphate Exposures Based on Selective Zirconia Adsorption and Enzyme-Catalytic Precipitation." Biosensors and Bioelectronics 24(8):2377-2383.

Wang, J, and Y Lin. 2009. "Nanomaterial-Based Biosensors for Detection of Pesticides and Explosives." Chapter 26 in Nanotechnology Applications for Clean Water. ed. N Savage et al, pp. 377-390. William Andrew, Nowich, NY.

Wang, J, G Liu, H Wu, and Y Lin. 2009. "Biosensors Based on Functionalized Carbon Nanotubes, Nanoparticles, and Nanowires." Chapter 4 in Handbook of Electrochemical Nanotechnology. ed. Y Lin and HS Nalwa, Vol II, pp. 95-111. American Scientific Publishers, Stevenson Ranch, CA.

Wang, L, R Pal, W Huang, XC Zeng, and LS Wang. 2009. "Tuning the Electronic Properties of the Golden Buckyball by Endohedral Doping: M@Au16(-) (M=Ag,Zn, in).” Journal of Chemical Physics 130(5):Art. No. 051101.

Wang, L, W Huang, LS Wang, BB Averkiev, and AI Boldyrev. 2009, "Experimental and Theoretical Investigation of Three-Dimensional Nitrogen-Doped Aluminum Clusters AI8N- and AI8N." Journal of Chemical Physics 130(13):Art No.: 134303. 10.1063/1.3097761 
Wang, XB, AP Sergeeva, J Yang, X Xing, AI Boldyrev, and LS Wang. 2009, "Photoelectron Spectroscopy of Cold Hydrated Sulfate Clusters, $\left.\mathrm{SO}_{4}{ }^{2-} \mathrm{H}_{2} \mathrm{O}\right)_{N}((N=4-7)$ : Temperature-Dependent Isomer Populations." Journal of Physical Chemistry A 113(19):5567-76.

Wang, Y, L An, LV Saraf, CM Wang, V Shutthanandan, DE McCready, and S Thevuthasan. 2009, "Microstructure and Ionic-Conductivity of Alternating-Multilayer Structured Gd-Doped Ceria and Zirconia Thin Films.” Journal of Materials Science 44(8):2021-26.

Warner, MG, JW Grate, AJ Tyler, RM Ozanich, KD Miller, J Lou, JD Marks, and CJ Bruckner-Lea. 2009. "Quantum Dot Immunoassays in Renewable Surface Column and 96-Well Plate Formats for the Fluorescence Detection of Botulinum Neurotoxin Using High-Affinity Antibodies." Biosensors and Bioelectronics 25(1):179-84.

Webb-Robertson, B-JM, LA McCue, N Beagley, JE McDermott, DS Wunschel, SM Varnum, JZ Hu, NG Isern, GW Buchko, K McAteer, JG Pounds, SJ Skerret, D Liggitt, and CW Frevert. 2009. "A Bayesian Integration Model of High-Throughput Proteomics and Metabolomics Data for Improved Early Detection of Microbial Infections." in Pacific Symposium on Biocomputing, vol 14, 451-463. World Scientific Publishing Co.,

Wegener KM, EA Welsh, LE Thornton, NS Keren, JM Jacobs, KK Hixson, ME Monroe, DG Camp, II, RD Smith, and HB Pakrasi. 2008. "High Sensitivity Proteomics Assisted Discovery of a Novel Operon Involved in the Assembly of Photosystem II, a Membrane Protein Complex." Journal of Biological Chemistry 283(41):27829-27837. doi:10.1074/jbc.M803918200

Wietsma, TW, M Oostrom, MA Covert, TE Queen, and MJ Fayer. 2009. "An Automated Tool for Three Types of Saturated Hydraulic Conductivity Laboratory Measurements." Soil Science Society of America Journal 73(2):466-470.

Wigginton, NS, KM Rosso, AG Stack, and MF Hochella. 2009. "Long-Range Electron Transfer across Cytochrome-Hematite (a-Fe2o3) Interfaces." Journal of Physical Chemistry C 113(6):2096-2103.

Wiley, HS. 2009. "Facts First.” The Scientist 23(2):29.

Wiley, HS. 2009. “The Problem of Perception.” The Scientist 23(3):31.

Williams, KH, A Kemna, MJ Wilkins, JL Druhan, EV Arntzen, AL N'Guessan, PE Long, SS Hubbard, and JF Banfield. 2009. "Geophysical Monitoring of Coupled Microbial and Geochemical Processes During Stimulated Subsurface Bioremediation.” Environmental Science \& Technology 43(17):6717â€”23.

Windisch, CF, CH Henager, MH Engelhard, and WD Bennett. 2009. "Raman and XPS Characterization of Fuel-Cladding Interactions Using Miniature Specimens." Journal of Nuclear Materials 383(3):237243.

Windus, TL, EJ Bylaska, K Tsemekhman, J Andzelm, and N Govind. 2009, "Computational Nanoscience with NWChem." Journal of Computational and Theoretical Nanoscience 6(6 SP ISS):1297-304. 
Wu H, Q Huo, SM Varnum, G Liu, J Wang, Z Nie, J Liu, and Y Lin. 2008. "Dye-Doped Silica Nanoparticle Labels/Protein Microarray for Detection of Protein Biomarkers." The Analyst 133:1550 1555. doi:10.1039/b719810h

Wu, H, J Wang, X Kang, CM Wang, D Wang, J Liu, IA Aksay, and Y Lin. 2009. "Glucose Biosensor Based on Immobilization of Glucose Oxidase in Platinum Nanoparticles/Graphene/Chitosan Nanocomposite Film.” Talanta 80(1):403-06.

Wu, S, F Yang, R Zhao, N Tolic, EW Robinson, DG Camp, RD Smith, and L Pasa-Tolic. 2009, “An Integrated Workflow for Characterizing Intact Phosphoproteins from Complex Mixtures." Analytical Chemistry 81(11):4210-19. 10.1021/ac802487q

Wu, S, NM Lourette, N Tolic, R Zhao, R Robinson, AV Tolmachev, RD Smith, and L Pasa-Tolic. 2009. "An Integrated Top-Down and Bottom-up Strategy for Broadly Characterizing Protein Isoforms and Modifications.” Journal of Proteome Research 8(3):1347-1357.

Xiang X, XT Zu, S Zhu, LM Wang, V Shutthanandan, P Nachimuthu, and Y Zhang. 2008. "Photoluminescence of $\mathrm{SnO} 2$ nanoparticles embedded in A12O3." Journal of Physics D. Applied Physics 41(22):225102. doi:10.1088/0022-3727/41/22/225102

Xiao, HY, F Gao, XT Zu, and WJ Weber. 2009, "Threshold Displacement Energy in Gan; Ab Initio Molecular Dynamics Study." Journal of Applied Physics 105(12):123527, 1-5. 10.1063/1.3153277

Xing X, P Wang, B Reed, SJ Baek, and CY Ng. 2008. "Infrared Vacuum-Ultraviolet Laser Pulsed Field Ionization-Photoelectron Study of $\mathrm{CH}_{3} \mathrm{Br}^{+}\left(\mathrm{X}^{\sim}\right.$ 2E3/2)." Journal of Physical Chemistry A 112(39):92779282. doi:10.1021/jp8019649

Xiong Z, YS Chua, C Ping, G Wu, WJ Shaw, AJ Karkamkar, JC Linehan, TD Smurthwaite, and T Autrey. 2008. "Interaction of lithium hydride and ammonia borane in THF ." Chemical Communications 43:5595-5597. doi:10.1039/b812576g

Xu, Z, P Meakin, and AM Tartakovsky. 2009. "Diffuse-Interface Model for Smoothed Particle Hydrodynamics." Physical Review. E, Statistical Physics, Plasmas, Fluids, and Related Interdisciplinary Topics 79(3):Art. No. 036702.

Yabusaki SB, Y Fang, and SR Waichler. 2008. "Building Conceptual Models of Field-Scale Uranium Reactive Transport in a Dynamic Vadose Zone-Aquifer-River System." Water Resources Research 44:W12403. doi:10.1029/2007WR006617

Yan, P, T Wang, GJ Newton, TV Knyushko, Y Xiong, DJ Bigelow, TC Squier, and MU Mayer. 2009, “A Targeted Releasable Affinity Probe (Trap) for in Vivo Photo-Crosslinking." Chembiochem 10(9):150718. 10.1002/cbic.200900029

Yang Y, C Mims, RS Disselkamp, D Mei, JH Kwak, J Szanyi, CHF Peden, and CT Campbell. 2008. "Isotope effects in methanol synthesis and the reactivity of copper formates on a $\mathrm{Cu} / \mathrm{SiO} 2$ catalyst." Catalysis Letters 125(3-4):201-208. doi:10.1007/s10562-008-9592-4 
Yang Z, ER Vorpagel, and J Laskin. 2008. "Experimental and Theoretical Studies of the Structures and Interactions of Vancomycin Antibiotics with Cell Wall Analogues." Journal of the American Chemical Society 130(39):13013-13022. doi:10.1021/ja802643g

Yang, F, S Wu, DL Stenoien, R Zhao, ME Monroe, MA Gritsenko, SO Purvine, AD Polpitiya, N Tolic, Q Zhang, AD Norbeck, DJ Orton, RJ Moore, K Tang, GA Anderson, L Pasa-Tolic, DG Camp, and RD Smith. 2009, "Combined Pulsed-Q Dissociation and Electron Transfer Dissociation for Identification and Quantitation of iTRAQ-labeled Phosphopeptides.” Analytical Chemistry 81(10):4137-43.

$10.1021 / \mathrm{ac} 802605 \mathrm{~m}$

Yang, J, M Stewart, GD Maupin, DR Herling, and A Zelenyuk. 2009, "Single Wall Diesel Particulate Filter (DPF) Filtration Efficiency Studies Using Laboratory Generated Particles." Chemical Engineering Science 64(8):1625-34.

Yang, Z, ER Vorpagel, and J Laskin. 2009. "Influence of the Charge State on the Structures and Interactions of Vancomycin Antibiotics with Cell-Wall Analogue Peptides: Experimental and Theoretical Studies." Chemistry - a European Journal 15(9):2081-2090.

Yi, C-W, and J Szanyi. 2009. "Interaction of D2o with a Thick BaO Film: Formation of and Phase Transitions in Barium Hydroxides.” Journal of Physical Chemistry C 113(35):15692-97.

Yi, C-w, and J Szanyi. 2009. "Reaction of NO2 with a Pure, Thick BaO Film: The Effect of Temperature on the Nature of NOx Species Formed." Journal of Physical Chemistry C 113(6):2134-2140.

Yi, C-WW, and J Szanyi. 2009. "BaO/Al2o3/NiAl(110) Model NOx Storage Materials: The Effect of $\mathrm{BaO}$ Film Thickness on the Amorphous-to-Crystalline Ba(NO3)2 Phase Transition." Journal of Physical Chemistry C 113(2):716-723.

Yoo, S, MV Kirov, and SS Xantheas. 2009, "Low-Energy Networks of the T-Cage (H2O)(24) Cluster and Their Use in Constructing Periodic Unit Cells of the Structure I (sl) Hydrate Lattice." Journal of the American Chemical Society 131(22):7564-66.

Yoo, S, XC Zeng, and SS Xantheas. 2009, "On the Phase Diagram of Water with Density Functional Theory Potentials: The Melting Temperature of Ice I-h with the Perdew-Burke-Ernzerhof and Becke-LeeYang-Parr Functionals.” Journal of Chemical Physics 130(22):Art. No. 211102. 10.1065/1.3153871

Yu, Y, ML Alexander, V Perraud, E Bruns, S Johnson, M Ezell, and BJ Finlayson-Pitts. 2009, "Contamination from Electrically Conductive Silicone Tubing During Aerosol Chemical Analysis." Atmospheric Environment 43(17):2836-39. 10.1016/j.atmosenv.2009.02.014

Zarzycki, PP, and KM Rosso. 2009, "Origin of Two Time-Scale Regimes in Potentiometric Titration of Metal Oxides. A Replica Kinetic Monte Carlo Study.” Langmuir 25(12):6841-48.

Zelenyuk A, J Yang, C Song, RA Zaveri, and DG Imre. 2008. "A New Real-Time Method for Determining Particles Sphericity and Density: Application to Secondary Organic Aerosol Formed by Ozonolysis of alpha-Pinene." Environmental Science \& Technology 42(21):8033-8038. doi:10.1021/es8013562 
Zelenyuk, A, and D Imre. 2009. "Beyond Single Particle Mass Spectrometry: Multidimensional Characterisation of Individual Aerosol Particles." International Reviews in Physical Chemistry 28(2):30958.

Zelenyuk, A, J Yang, and DG Imre. 2009, "Comparison between Mass Spectra of Individual Organic Particles Generated by UV Laser Ablation and in the IR/UV Two-Step Mode." International Journal of Mass Spectrometry 282(1-2):6-12.

Zelenyuk, A, J Yang, DG Imre, and EY Choi. 2009, “Achieving Size Independent Hit-Rate in Single Particle Mass Spectrometry.” Aerosol Science and Technology 43(4):305-10.

Zelenyuk, A, J Yang, EY Choi, and DG Imre. 2009, "Splat II: An Aircraft Compatible, Ultra-Sensitive, High Precision Instrument for in-Situ Characterization of the Size and Composition of Fine and Ultrafine Particles.” Aerosol Science and Technology 43(5):411-24.

Zhai, HJ, B Wang, X Huang, and LS Wang. 2009, "Probing the Electronic and Structural Properties of the Niobium Trimer Cluster and Its Mono- and Dioxides: $\mathrm{Nb}_{3} \mathrm{O}_{n}{ }^{-}$and $\mathrm{Nb}_{3} \mathrm{O}_{n}(n=0-2)$." Journal of Physical Chemistry A 113(16):3866-75.

Zhang Q, AA Schepmoes, JW Brock, S Wu, RJ Moore, SO Purvine, J Baynes, RD Smith, and TO Metz. 2008. "Improved Methods for the Enrichment and Analysis of Glycated Peptides ." Analytical Chemistry 80(24):9822-9829. doi:10.1021/ac801704j

Zhang Q, VA Petyuk, AA Schepmoes, DJ Orton, ME Monroe, F Yang, RD Smith, and TO Metz. 2008. "Analysis of Non-Enzymatically Glycated Peptides: Neutral-Loss Triggered MS3 Versus Multi-Stage Activation Tandem Mass Spectrometry." Rapid Communications in Mass Spectrometry 22(19):30273034. doi: $10.1002 / \mathrm{rcm} .3703$

Zhang Z, RJ Rousseau, J Gong, SC Li, BD Kay, Q Ge, JM White, and Z Dohnalek. 2008. "Vacancy Assisted Diffusion of Alkoxy Species on Rutile TiO2(110).” Physical Review Letters 101(15):156103. doi:10.1103/PhysRevLett.101.156103

Zhang, G, H Dong, H Jiang, RK Kukkadapu, J Kim, DD Eberl, and Z Xu. 2009. "Biomineralization Associated with Microbial Reduction of Fe3+ and Oxidation of Fe2+ in Solid Minerals." American Mineralogist 94(7):1049-58.

Zhang, H, X Tang, GR Munske, N Tolic, GA Anderson, and JE Bruce. 2009. "Identification of ProteinProtein Interactions and Topologies in Living Cells with Chemical Cross-Linking and Mass Spectrometry." Molecular \& Cellular Proteomics. MCP 8(3):409-420.

Zhang, M, RC Ewing, LA Boatner, EKH Salje, WJ Weber, P Daniel, Y Zhang, and I Farnan. 2009, "Pb+ Irradiation of Synthetic Zircon (ZrSiO4) Infrared Spectroscopic Investigation - Reply.” American Mineralogist 94(5-6):856-58. 10.2138/am.2009.542

Zhang, Q, LV Saraf, JR Smith, P Jha, and F Hua. 2009, “An Invisible Bend Sensor Based on Porous Crosslinked Polyelectrolyte Film.” Sensors and Actuators. A, Physical 151(2):154-58. 10.1016/j.sna.2009.02.034 
Zhang, Y, and WJ Weber. 2009, "Response of Materials to Single Ion Events." Nuclear Instruments and Methods in Physics Research. Section B, Beam Interactions with Materials and Atoms 267(8-9):1705-12.

Zhang, Y, I-T Bae, K Sun, CM Wang, M Ishimaru, Z Zhu, W Jiang, and WJ Weber. 2009, "Damage Profile and Ion Distribution of Slow Heavy Ions in Compounds." Journal of Applied Physics 105(10):104901:1-12. 10.1063/1.3118582

Zhang, Y, J Lian, Z Zhu, WD Bennett, LV Saraf, JL Rausch, CA Hendricks, RC Ewing, and WJ Weber. 2009, "Response of Strontium Titanate to Ion and Electron Irradiation." Journal of Nuclear Materials 389(2):303-10.

Zhang, Z, Y Du, NG Petrik, GA Kimmel, I Lyubinetsky, and Z Dohnalek. 2009. "Water as a Catalyst: Imaging Reactions of O-2 with Partially and Fully Hydroxylated TiO2(110) Surfaces." Journal of Physical Chemistry C 113(5):1908-1916.

Zhao, R, S-J Ding, Y Shen, DG Camp, EA Livesay, HR Udseth, and RD Smith. 2009. "Automated Metal-Free Multiple-Column NanoLC for Improved Phosphopeptide Analysis Sensitivity and Throughput." Journal of Chromatography B 877(8-9):663-670.

Zhou, Y, XT Zu, F Gao, JL Nie, and H Xiao. 2009. "Adsorption of Hydrogen on Boron-Doped Graphene: A First-Principles Prediction.” Journal of Applied Physics 105(1):014309.

Zhu, K, JZ Hu, X She, J Liu, Z Nie, Y Wang, CHF Peden, and JH Kwak. 2009. "Characterization of Dispersed Heteropoly Acid on Mesoporous Zeolite Using Solid-State P-31 NMR Spin-Lattice Relaxation." Journal of the American Chemical Society 131(28):9715-21.

\section{Book Chapters}

Bond DL, JA Davis, and JM Zachara. 2008. "Uranium(VI) Release from Contaminated Vadose Zone Sediments: Estimation of Potential Contributions from Dissolution and Desorption." In Adsorption of Metals to Geomedia II, ed. M.O. Barnett, D. B. Kent, pp. 379-420. Academic Press, San Diego, CA.

Grate, JW, MH Abraham, and BM Wise. 2009, "Design and Information Content of Arrays of SorptionBased Vapor Sensors Using Solubility Interactions and Linear Solvation Energy Relationships." in Selection of Materials for Chemical Vapor Sensing Arrays, eds. M Ryan, et al., pp. 193-220. CRC Press, Boca Raton, FL.

Lipton, AS, PD Ellis, and TE Polenova. 2009, "Quadrupolar Metal Nuclides in Bioinorganic Chemistry: Solid-State NMR Studies." in Encyclopedia of Magnetic Resonance. John Wiley \& Sons, Hoboken, NJ.

Majors PD, and JS Mclean. 2008. "Dynamic Metabolism Studies of Live Bacterial Films." Chapter 22 in Magnetic Resonance Microscopy, Codd \& Seymour, Eds., ed. Sarah Codd and Joseph D. Seymour Wiley-VCH, Weinheim, Belgium, Germany.

Wang, XB, and LS Wang. 2009, "Photoelectron Spectroscopy of Multiply Charged Anions." in Annual Review of Physical Chemistry, Vol 60, pp. 105-26. Annual Reviews, Inc., Palo Alto, CA. 